\title{
Procedures and Best Practices for Trigonometric Leveling in the U.S. Geologicall Survey
}


Cover Front, top: U.S. Geological Survey scientist sighting a prism with a total station for a survey of a hydraulic structure on the West Aspetuck River, Connecticut. Photograph by Caelan Simeone. Front, bottom right: U.S. Geological Survey scientist measuring a cross section of the Hop River with a robotic total station and prism pole, Connecticut. Photograph by Michael Noll. Front, bottom left: U.S. Geological Survey technician setting up a fixed-height tripod and prism for a vertical-control traverse, Cataract Canyon, Utah. Photograph by Michael Freeman. Back, top: U.S. Geological Survey land survey crew with surveying transit and targets. Photographer is unknown. Back, bottom right: U.S. Geological Survey land surveyor using plane table and alidade for mapping purposes in Alaska. Photograph by Bruce Geyman. Back, bottom left: U.S. Geological Survey land surveyor using plane table and alidade for mapping purposes in the southwestern United States. Photograph by Bruce Geyman. 


\section{Procedures and Best Practices for Trigonometric Leveling in the U.S. Geological Survey}

By Michael L. Noll and Paul H. Rydlund, Jr.

Techniques and Methods 11-D3 


\title{
U.S. Department of the Interior \\ DAVID BERNHARDT, Secretary
}

\author{
U.S. Geological Survey \\ James F. Reilly II, Director
}

U.S. Geological Survey, Reston, Virginia: 2020

For more information on the USGS - the Federal source for science about the Earth, its natural and living resources, natural hazards, and the environment—visit https://www.usgs.gov or call 1-888-ASK-USGS.

For an overview of USGS information products, including maps, imagery, and publications, visit https://store.usgs.gov/.

Any use of trade, firm, or product names is for descriptive purposes only and does not imply endorsement by the U.S. Government.

Although this information product, for the most part, is in the public domain, it also may contain copyrighted materials as noted in the text. Permission to reproduce copyrighted items must be secured from the copyright owner.

Suggested citation:

Noll, M.L., and Rydlund, P.H., 2020, Procedures and best practices for trigonometric leveling in the U.S. Geological Survey: U.S. Geological Survey Techniques and Methods, book 11, chap. D3, 94 p., https://doi.org/10.3133/tm11D3.

ISSN 2328-7047 (print)

ISSN 2328-7055 (online) 


\section{Contents}

Abstract

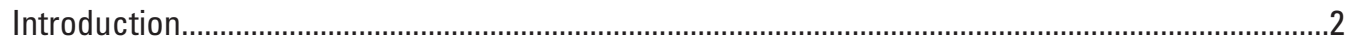

Benefits and Limitations of Trigonometric Leveling .................................................................

Current Applications of Total Station Instruments in the U.S. Geological Survey....................4

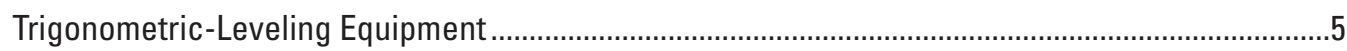

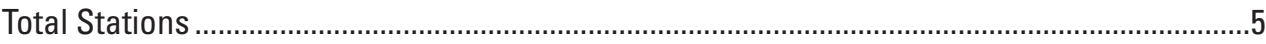

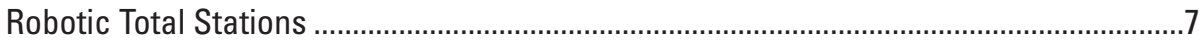

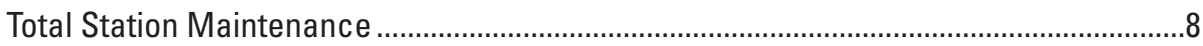

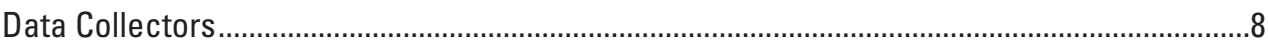

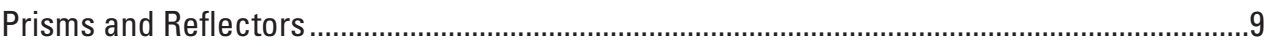

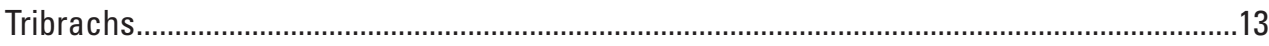

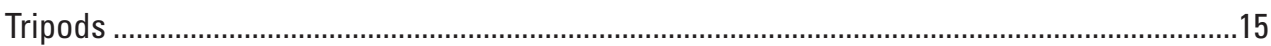

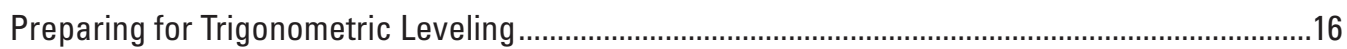

Level Bubble and Plummet Adjustments ............................................................................16

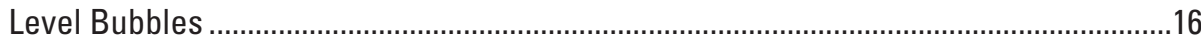

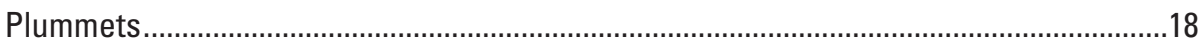

Testing Total Station Components Before a Trigonometric-Leveling Campaign......................21

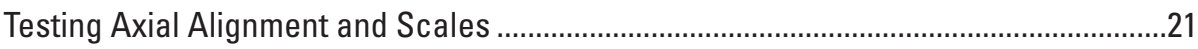

Vertical Collimation Error........................................................................................22

Horizontal Collimation Error ………….................................................................26

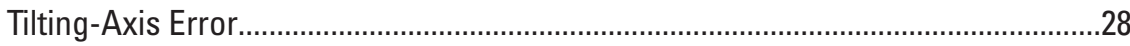

Vertical-Axis Error (Tilt of the Vertical Axis) ..........................................................32

Testing the Electronic Distance Measurement Instrument..............................................32

Electronic Distance Measurement Instrument Testing Procedure at National Geodetic Survey Calibrated Baselines.............................................35

Sources of Measurement Uncertainty for Trigonometric Leveling .................................................3

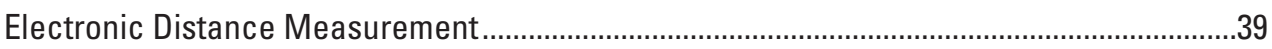

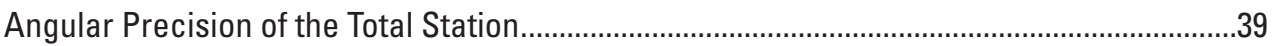

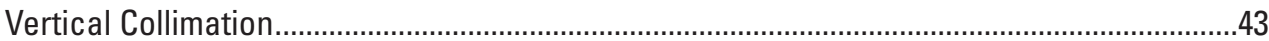

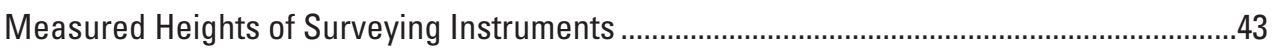

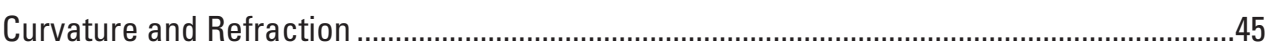

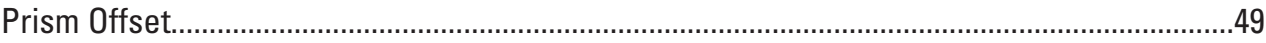

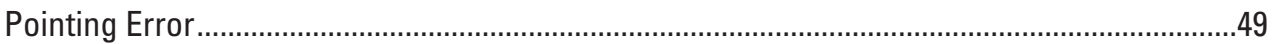

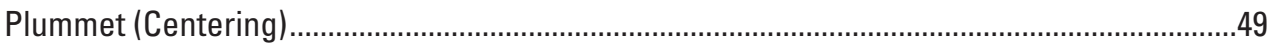

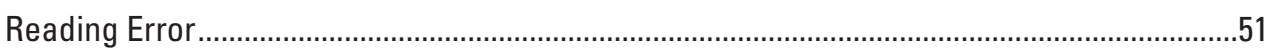

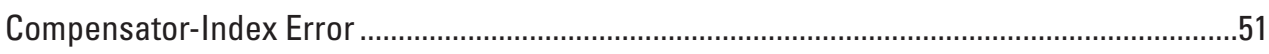

Meteorological Measurements (Temperature and Pressure) ………....................................51

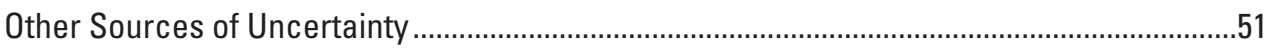

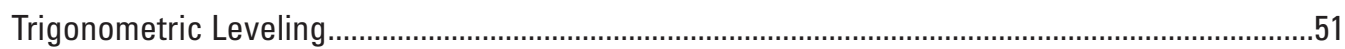

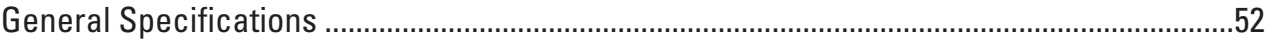

Establishing an Origin Point for the Survey ..........................................................................

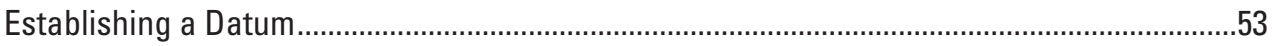

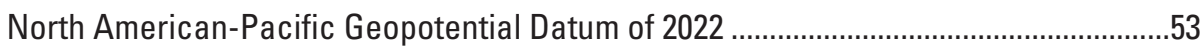

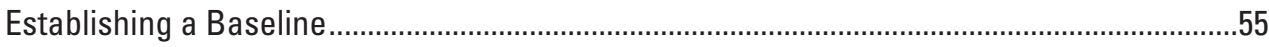


Direct and Reverse Instrument Orientation Measurements ................................................56

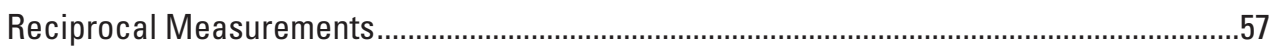

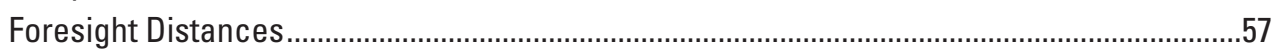

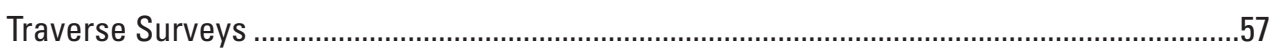

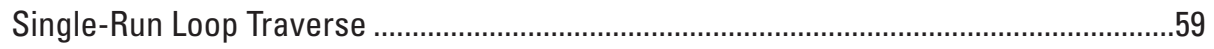

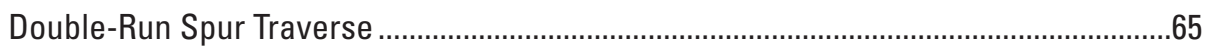

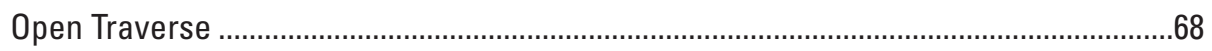

Adjustments of Field Measurements.........................................................................72

Determining Section Misclosure from Reciprocal Observations ............................72

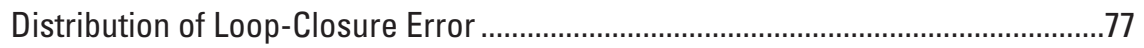

Determining Section Misclosure from Double-Run Observations ..........................78

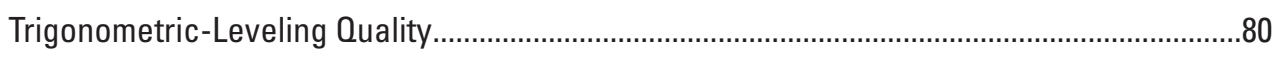

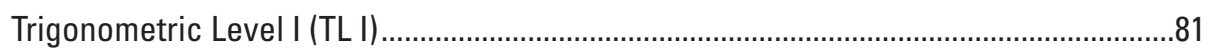

Trigonometric Level II (TL II) ............................................................................ 81

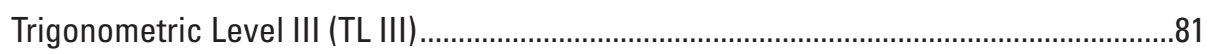

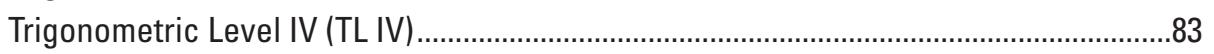

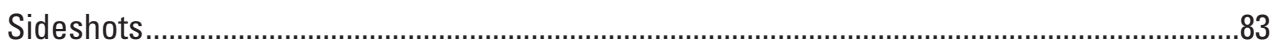

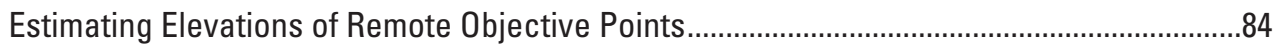

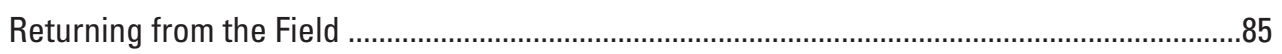

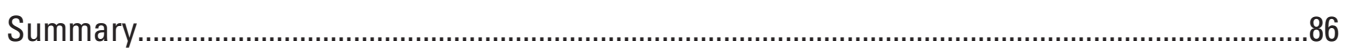

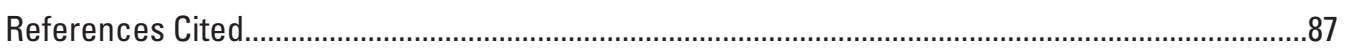

Appendix 1 Standard Field Form for Running Trigonometric Levels ............................................91

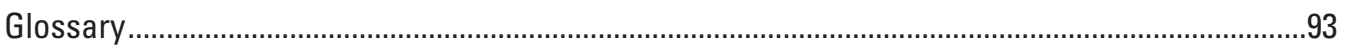

\section{Figures}

1. Diagrams showing terrestrial-leveling methods for variable topography terrain .............3

2. Diagrams and photographs showing prism equipment used for surveying ....................10

3. Photographs showing trigonometric levels surveying equipment..................................14

4. Photographs showing the method to calibrate a bubble tube level of a total station ....17

5. Photographs showing circular level vial adjustment on fixed-height center poles........19

6. Photographs and diagram showing the method to adjust rotating optical or

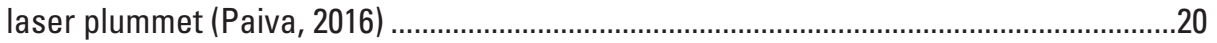

7. Diagram showing the orientation of three instrument axes of a total station..................22

8. Diagram showing the geometry of a total station measurement ....................................23

9. Diagrams showing common systematic sources of error for total station

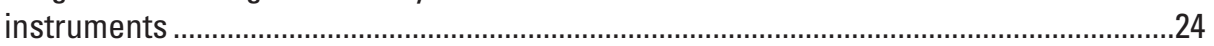

10. Diagrams showing the method for determining vertical collimation error of a total station.

11. Diagrams showing the method for determining horizontal collimation error of a total station

12. Graph showing potential elevation error resulting from a horizontal positional error of plus or minus 0.102 feet on a slope with a downslope angle of about 50 degrees.

13. Diagrams showing the method for determining tilting-axis error of a total station ........30 
14. Graph showing the total uncertainty of an electronic distance measurement for a total station with a constant error of 2 millimeters and a distance-weighted

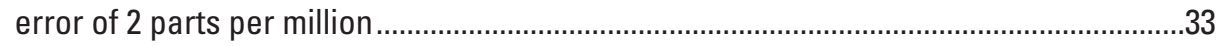

15. Map showing the location of National Geodetic Survey calibrated baselines.................34

16. Diagram showing the National Geodetic Survey calibrated baseline configuration at Hemsptead, New York.

17. Image showing an example of a National Geodetic Survey baseline calibration sheet for Hempstead, New York.....................................................................................

18. Graph and diagram showing implications of electronic distance measurement uncertainty on the vertical difference.............................................................................

19. Photographs showing the relation between the slant height and the instrument height.

20. Diagram showing the combined measurement uncertainty for Earth's curvature and atmospheric refraction, and a data-collection device screen.................................46

21. Diagram showing the trigonometric levels decision tree and survey process...............54

22. Diagrams showing closed and open traverse surveys ................................................58

23. Diagrams and images showing a hypothetical single-run loop traverse........................61

24. Diagram showing a hypothetical double-run spur traverse............................................66

25. Diagrams showing the hypothetical open traverse and modified double-rod method.

26. Diagrams showing field measurements of a single-run loop traverse and the procedure to calculate section misclosure from reciprocal observations, determine preliminary mark-to-mark vertical differences, and determine

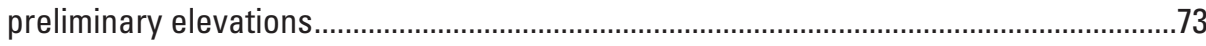

27. Diagrams showing the leveling process for a double-run spur traverse.......................79

28. Diagram showing a hypothetical open traverse and four sideshot surveys ....................84

29. Diagram showing estimating the elevation of remote objects using surveying instruments and simple trigonometry

\section{Tables}

1. Linear error caused by angular uncertainty of the total station at measurement distances of 200,500, and 1,000 feet along a horizontal line of sight................................7

2. Sources of measurement uncertainty for a trigonometric-leveling survey.....................40

3. Estimated maximum potential vertical difference uncertainty for a total station with 3 arc-second angular precision and an electronic distance measurement instrument uncertainty of 2 millimeters plus 2 parts per million.

4. Estimated measurement uncertainty caused by Earth's curvature and atmospheric refraction for foresight distances of 100, 200, 500, 1,000, 2,500, 5,280 and 10,560 feet.

5. Recommendations for trigonometric levels ..........................................................50

6. Summary of criteria for closed and open traverses ...................................................71

7. Preliminary and final elevations of a single-run loop traverse ......................................78

8. Accuracy standards for vertical-control networks and corresponding example specifications

9. Trigonometric-leveling standards and corresponding specifications for field procedures 


\section{Conversion Factors}

U.S. customary units to International System of Units

\begin{tabular}{lcll}
\hline & Multiply & By & \multicolumn{1}{c}{ To obtain } \\
\hline inch (in.) & 2.54 & centimeter $(\mathrm{cm})$ \\
inch (in.) & 25.4 & millimeter (mm) \\
foot (ft) & 0.3048 & meter $(\mathrm{m})$ \\
mile (mi) & 1.609 & kilometer $(\mathrm{km})$ \\
\hline
\end{tabular}

Temperature in degrees Celsius $\left({ }^{\circ} \mathrm{C}\right)$ may be converted to degrees Fahrenheit $\left({ }^{\circ} \mathrm{F}\right)$ as ${ }^{\circ} \mathrm{F}=(1.8 \times$ $\left.{ }^{\circ} \mathrm{C}\right)+32$.

Temperature in degrees Fahrenheit $\left({ }^{\circ} \mathrm{F}\right)$ may be converted to degrees Celsius $\left({ }^{\circ} \mathrm{C}\right)$ as ${ }^{\circ} \mathrm{C}=\left({ }^{\circ} \mathrm{F}-\right.$ 32) / 1.8 .

\section{Datum}

Vertical coordinate information is referenced to the North American Vertical Datum of 1988 (NAVD 88).

Horizontal coordinate information is referenced to the North American Datum of 1983 (NAD 83).

Elevation, as used in this manual, is a height referenced to a vertical datum.

\section{Supplemental Information}

U.S. customary feet (ft) may be converted to U.S. survey feet ( $\mathrm{stt}$ ) as follows: $\mathrm{fft}=\left(\mathrm{ft} \times 2 \times 10^{-6}\right)-\mathrm{ft}$

U.S. customary units are the primary units used in this report; however, the International System of Units are used for (1) construction details and equipment specifications for surveying instrumentation because most manufacturers build their products according to the International System of Units, (2) information from the National Geodetic Survey to preserve the integrity of the primary-source data (for example, publications and information on calibrated baselines), and (3) in some cases, conversions to U.S. units require carrying the conversion to the sixth decimal place or more, which may be extraneous and confusing for the reader.

Angular precision or uncertainty is given in arc-seconds ().

Device precision or uncertainty is given in millimeters $(\mathrm{mm})$ plus or minus parts per million ( $\mathrm{ppm})$ or in millimeters $(\mathrm{mm})$ plus millimeters per kilometer $(\mathrm{mm} / \mathrm{km})$. 


\section{Abbreviations}

$\begin{array}{ll}\text { ATR } & \text { automatic target recognition } \\ \text { DRST } & \text { double-run spur traverse } \\ \text { EDM } & \text { electronic distance measurement } \\ \text { EDMI } & \text { electronic distance measurement instrument } \\ \text { F1 } & \text { total station is in direct instrument orientation } \\ \text { F2 } & \text { total station is in reverse instrument orientation } \\ \text { FGCC } & \text { Federal Geodetic Control Committee } \\ \text { GNSS } & \text { Global Navigation Satellite System } \\ \text { HI } & \text { height of instrument } \\ \text { LCE } & \text { loop-closure error } \\ \text { NGS } & \text { National Geodetic Survey } \\ \text { NOAA } & \text { National Oceanic and Atmospheric Administration } \\ \text { OT } & \text { open traverse } \\ \text { SCE } & \text { spur-closure error } \\ \text { SD } & \text { slope distance } \\ \text { SH } & \text { height of sight } \\ \text { SRLT } & \text { single-run loop traverse } \\ \text { TL } & \text { Trigonometric Level } \\ \text { USACE } & \text { U.S. Army Corps of Engineers } \\ \text { USGS } & \text { U.S. Geological Survey } \\ \text { VD } & \text { vertical difference } \\ \text { WSC } & \text { water science center } \\ \text { ZA } & \text { zenith angle } \\ \end{array}$





\title{
Procedures and Best Practices for Trigonometric Leveling in the U.S. Geological Survey
}

\author{
By Michael L. Noll and Paul H. Rydlund, Jr.
}

\section{Abstract}

With the advent of highly precise total stations and modern surveying instrumentation, trigonometric leveling has become a compelling alternative to conventional leveling methods for establishing vertical-control networks and for perpetuating a datum to field sites. Previous studies of trigonometric-leveling measurement uncertainty proclaim that first-, second-, and third-order accuracies may be achieved if strict leveling protocols are rigorously observed. Common field techniques to obtain quality results include averaging zenith angles and slope distances observed in direct and reverse instrument orientation (F1 and F2, respectively), multiple sets of reciprocal observations, quality meteorological observations to correct for the effects of atmospheric refraction, and electronic distance measurements that generally do not exceed 500 feet. In general, third-order specifications are required for differences between F1 and F2 zenith angles and slope distances; differences between redundant instrumentheight measurements; section misclosure determined from reciprocal observations; and closure error for closed traverse. For F1 observations such as backsight check and check shots, the construction-grade specification is required for elevation differences between known and observed values.

Recommended specifications for trigonometric-leveling equipment include a total station instrument with an angular uncertainty specification less than or equal to plus or minus 5 arc-seconds equipped with an integrated electronic distance measurement device with an uncertainty specification of less than or equal to plus or minus 3 millimeters plus 3 parts per million. A paired data collector or integrated microprocessor should have the capability to average multiple sets of measurements in direct and reverse instrument orientation. Redundant and independent measurements by the survey crew and automated or manual reduction of slant heights to the vertical equivalent are recommended to obtain quality instrument heights. Horizontal and vertical collimation tests should be conducted daily during trigonometric-leveling surveys, and electronic distance-measurement instruments should be tested annually on calibrated baselines maintained by the National Geodetic Survey. Specifications that were developed by the National Geodetic Survey for geodetic leveling have been adapted by the U.S. Geological Survey (USGS) for the purpose of developing standards for trigonometric leveling, which are identified as USGS Trigonometric Level I (TL I), USGS Trigonometric Level II (TL II), USGS Trigonometric Level III (TL III), and USGS Trigonometric Level IV (TL IV). TL I, TL II, and TL III surveys have a combination of first, second, and third geodetic leveling specifications that have been modified for plane leveling. The TL III category also has specifications that are adapted from construction-grade standards, which are not recognized by the National Geodetic Survey for geodetic leveling. A TL IV survey represents a leveling approach that does not generally meet criteria of a TL I, TL II, or TL III survey.

Site conditions, such as highly variable topography, and the need for cost-effective, rapid, and accurate data collection in response to coastal or inland flooding have emphasized the need for an alternative approach to conventional leveling methods. Trigonometric leveling and the quality-assurance methods described in this manual will accommodate most site and environmental conditions, but measurement uncertainty is potentially variable and dependent on the survey method. Two types of closed traverse surveys have been identified as reliable methods to establish and perpetuate vertical control: the single-run loop traverse and double-run spur traverse. Leveling measurements for a double-run spur traverse are made in the forward direction from the origin to the destination and are then retraced along the same leveling route in the backward direction, from the destination to the origin. Every control point in a double-run spur traverse is occupied twice. Leveling measurements for a single-run loop traverse are made in the forward direction from the origin point to the destination, and then from the destination to the origin point, along a different leveling route. The only point that is redundantly occupied for the single-run loop traverse is the origin. An open traverse method is also considered an acceptable approach to establish and perpetuate vertical control if the foresight prism height is changed between measurement sets to ensure at least two independent observations. A modified version of leap-frog leveling is recommended for all traverse surveys because it reduces measurement uncertainty by forcing the surveying instrumentation into a level and centered condition over the ground point as the instrumentation is advanced to the objective. Sideshots are considered any radial measurement made from the total station that is not part of a traverse survey. F1 and F2 observations are recommended 
for sideshots measurements for projects that require precise elevations. Quality-assurance measurements made in F1 from the station to network-control points should be considered for surveys that require a high quantity of sideshots.

The accuracy of a trigonometric-leveling survey essentially depends on four components (1) the skill and experience of the surveyor, (2) the environmental or site conditions, (3) the surveying method, and (4) the quality of the surveying instrumentation. Although components one and two can sometimes be difficult to evaluate and be highly variable, the objective of this manual is to disseminate information needed to identify, maintain, and operate quality land-surveying instrumentation, and to document procedures and best practices for preparing and executing precision trigonometric-leveling surveys in the USGS.

\section{Introduction}

A common and fundamental part of earth-science studies and data collection within the various disciplines of the U.S. Geological Survey (USGS) is to accurately determine elevations of vertical control (see the definition of this and other terms in the "Glossary" section) for the purpose of referencing scientific data to a common vertical datum. Elevations are established through vertical-control networks to objective locations using three terrestrial-leveling methods that are identified by the National Geodetic Survey (NGS) as differential, trigonometric, and barometric (National Oceanic and Atmospheric Administration [NOAA], 2017a). All three leveling methods are used to determine a height difference between two points; however, project specifications, environmental conditions, and accuracy requirements typically help determine the best approach.

The differential-leveling method, also known as spirit levels or geometric levels or fixed-plane levels, are considered the most accurate of the three methods, requiring the surveying instrument to be in a fixed position, with a calibrated rod or staff at the backsight and foresight positions. The operator makes an observation on the staff at each position - the observation is the value on the calibrated staff where the line of sight of the fixed-plane optical level intersects the staff- the difference between the observations is considered the change in height between the backsight and foresight locations. Firstorder accuracy is routinely achieved using the differentialleveling method and is, subsequently, considered the standard for vertical-control surveys.

The trigonometric-leveling method is used to accurately determine the height difference (vertical difference or VD) between two points by multiplying the measured slope distance by the cosine of the measured zenith angle. The surveyor uses a tripod-mounted total station or theodolite equipped with an electronic distance measurement (EDM) instrument (EDMI) to observe a slope distance and zenith angle from the point of occupation, typically called the station. A reflector, or prism, is set up at the foresight and serves two purposes: first, the center of the prism is used as the pointing target for the zenith angle measurement; and second, the prism returns the transmitted EDM signal to the instrument for processing of the slope distance. Once the VD between the station and prism is trigonometrically determined, the heights of the instrument and prism above the ground points are applied to obtain the VD between the two ground points, also called the mark-tomark VD. Trigonometric leveling is considered less accurate than differential leveling but more economical because horizontal and vertical measurements can be made simultaneously; and in mountainous or in project areas with variable topography, it may be the only practical leveling method because sight distances are not limited by the horizontal line of sight (fig. 1). If rigorous field procedures are adhered to and if error sources stemming from the environment, instrument, and operation are accounted for, some literature sources state that third-order standards or better can be achieved (Whalem, 1985; Chrzanowski, 1989; Kozlowski, 1998; U.S. Army Corps of Engineers [USACE], 2007; Bjelotomić and others, 2011; Rydlund and Noll, 2017).

Barometric leveling is not as accurate as differential or trigonometric leveling, so it is primarily used for reconnaissance and exploratory surveys (NOAA, 2017a). Height (vertical) differences can be rapidly determined by measuring changes in atmospheric pressure at various elevations with a mercurial or aneroid barometer. Depending on project requirements, the trigonometric- or differential-leveling method may be used later to more precisely determine the VD between points.

\section{Benefits and Limitations of Trigonometric Leveling}

Trigonometric leveling is the preferred leveling method for use in project areas with variable-topography terrain because less instrument setups may be required to complete a survey when compared to the differential-leveling method (fig. 1). Kenney (2010) recognized that closure error from differential leveling may accumulate with each instrument setup, so uncertainty can be reduced by reducing the number of times the instrument is set up. The number of instrument setups are reduced for trigonometric leveling, as compared to differential leveling, because sight distances are not limited by the horizontal line of sight. Additionally, Kenney (2010) suggested balanced (equivalent) sight distances of about 150 feet (ft) for differential leveling to reduce measurement uncertainty from vertical collimation error (described in subsequent sections); for trigonometric leveling, if measurements are made in direct and reverse instrument orientation (referred to herein as face 1 and face 2, respectively, and abbreviated F1 and F2, respectively), uncertainty from vertical collimation error is reduced (or eliminated) regardless of sight distance. Moreover, on sloping terrain, the line of sight between the optical level and the calibrated staff can intersect varying temperatures 
A

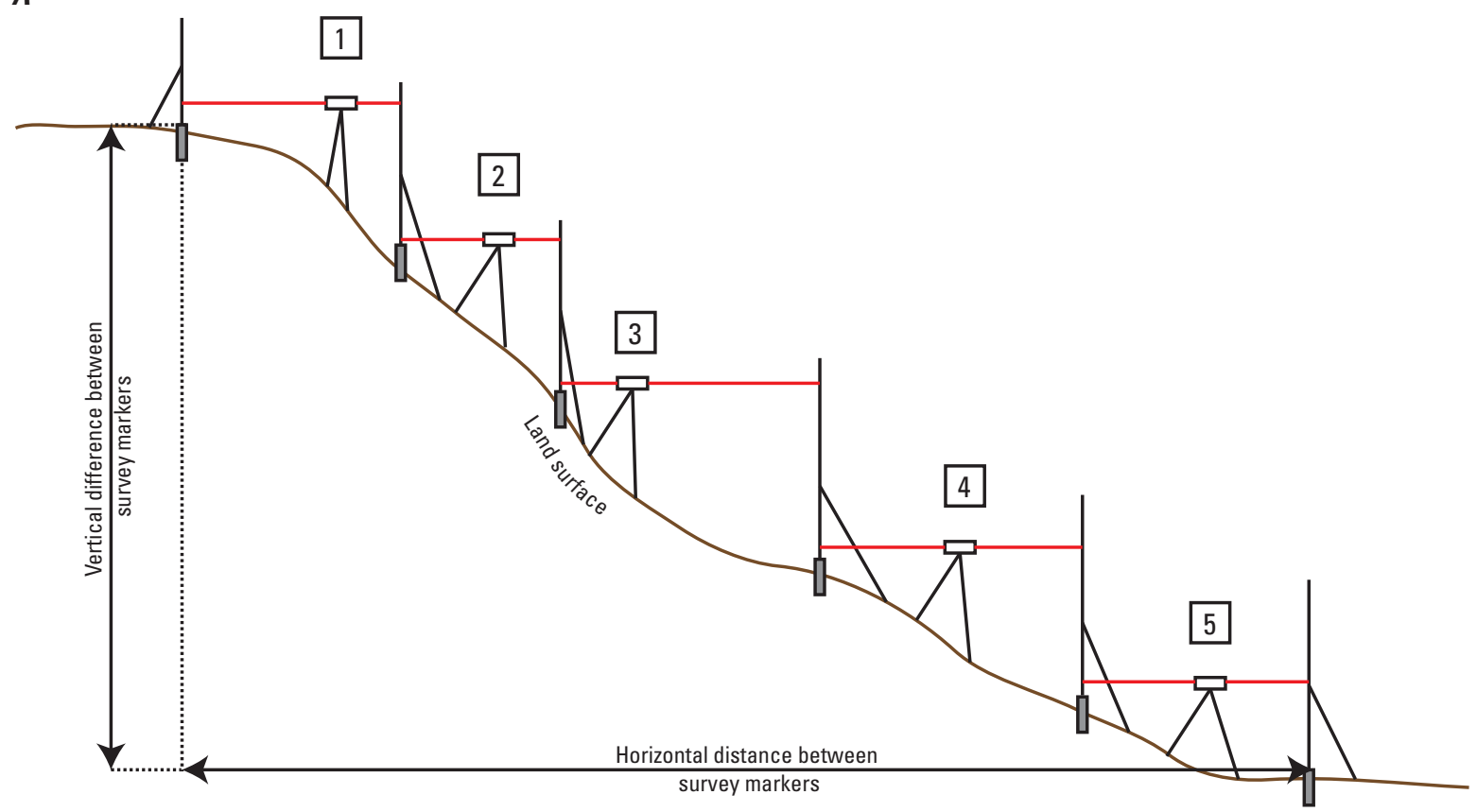

B 1

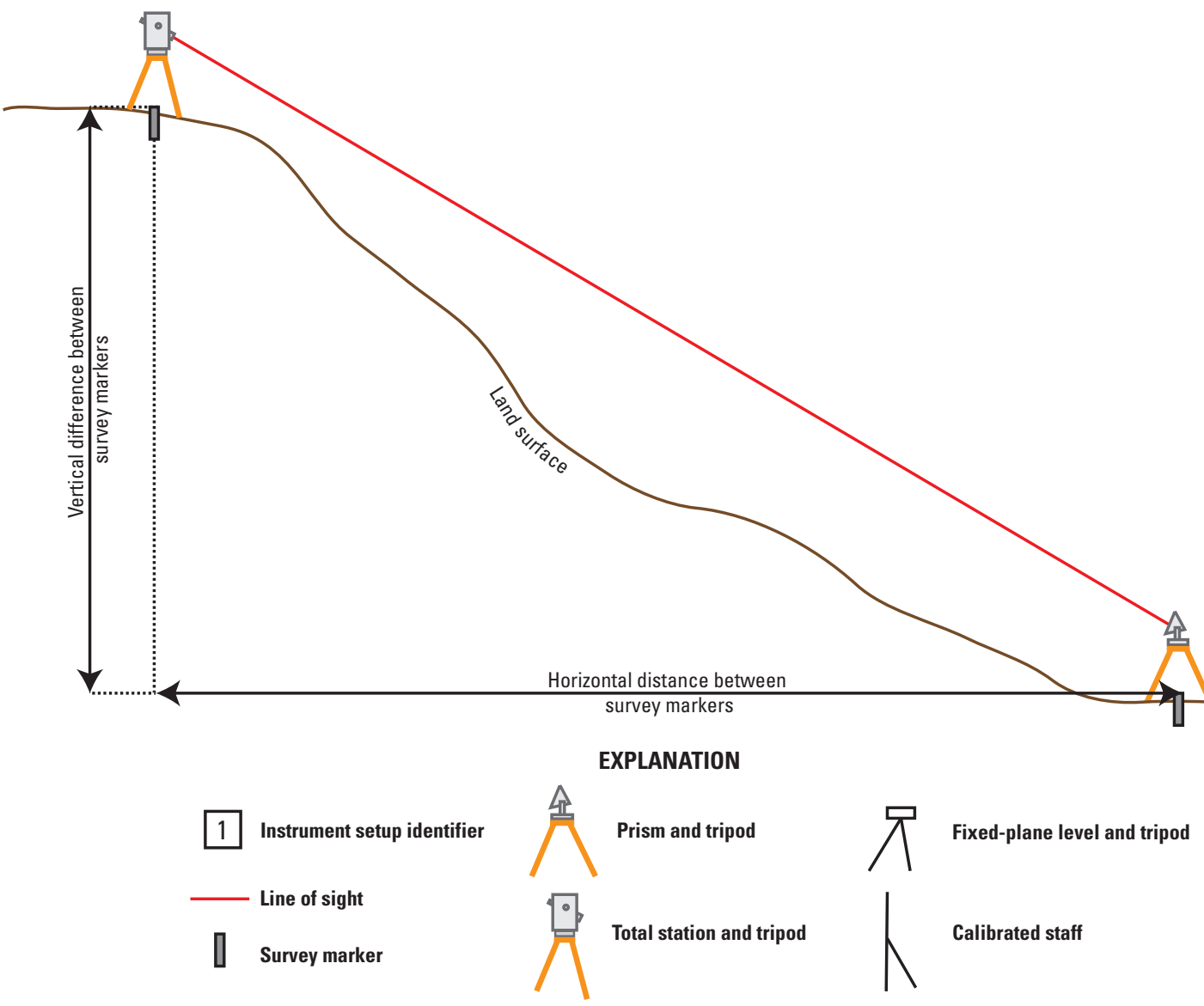

Figure 1. Terrestrial-leveling methods for variable topography terrain. $A$, differential leveling; and $B$, trigonometric leveling. 
(densities) of air, which may cause erroneous staff readings during differential leveling because of the effects of atmospheric refraction. During trigonometric-leveling surveys, the orientation of the telescope and the prism height can be adjusted to a line of sight that is roughly parallel to the sloped topography to reduce the effects of atmospheric refraction because horizontal temperature gradients tend to be parallel to land surface. Furthermore, literature indicates that third-order standards or better can be achieved with trigonometric leveling for foresight distances up to 1,000 ft (USACE, 2007; Rydlund and Noll, 2017). Foresight distances of less than $500 \mathrm{ft}$ are recommended in this manual because measurement uncertainty for trigonometric leveling is proportional to the measured slope distance. If this conservative estimate for foresight distance is used, the trigonometric-leveling method requires about one-third as many instrument setups per unit of horizontal distance surveyed compared to the number of setups for differential leveling.

If a total station equipped with an integrated microprocessor or paired, external data-collection device is used, slope and zenith angle measurements can be automatically processed and stored, along with important metadata such as instrument and prism heights. These devices can also automatically record and average measurements that are collected in F1 and F2, which are important parts of the procedure for reducing measurement uncertainty in trigonometric leveling. Automated data collection and processing increases work efficiency because the surveyor does not need to manually record angles and distances (or staff readings and barcode observations, in the case of differential leveling) for each measurement. Measurement uncertainty may also improve because transcription mistakes are reduced or eliminated. Horizontal measurements (angle and computed distance) can also be simultaneously collected with vertical measurements, so a plot of the survey and a record of measured distances are automatically created without the need for laborious field sketches. Automated data collection and processing have streamlined trigonometric leveling so that it may be a more economic and efficient way to collect precision elevation measurements compared to traditional methods. Robotic total stations have also reduced sources of measurement uncertainty related to the human (operator) component of the error budget such as pointing and reading errors. Robotic surveys also require smaller crews for field work (sometimes only a single surveyor), which could increase work efficiency by allowing survey crews to multitask during field work.

A fundamental goal of all precision leveling campaigns within the USGS is to reduce measurement uncertainty to achieve the highest level of accuracy possible. Like differential leveling, the precision of a trigonometric-leveling survey depends on the surveying instruments, the surveying method or procedure, the environmental conditions, and the skill of the surveyor. Any one of these factors can limit the precision of a trigonometric-leveling survey. Thus, to obtain quality measurements, a well-maintained and precisely adjusted surveying instrument should be operated by an experienced surveyor who applies a method that reduces uncertainty caused by systematic and random error sources that may stem from the environment, equipment, or surveyor and who minimizes possibilities of blunders.

When compared to differential leveling, trigonometric leveling requires more complex surveying instruments (total station) that are recognized as imperfect surveying instruments by the manufacturer for even the most high-end models. The angular precision of survey-grade total stations can range from 0.1 to 5 arc-seconds ("), and construction-grade models have an angular precision that exceeds 5". EDMIs also have a constant and distance-weighted uncertainty that can affect the measured slope distance and has implications on the derived VD. Corner-cube prisms are used as surveying targets for the total station and also have manufacturer defects that can cause the EDM signal to diverge from the ideal flight path resulting in an erroneous slope distance measurement and have a prism constant that may be different from the nominal published value provided by the manufacturer. These are just a few of many sources of systematic uncertainty that can affect the repeatability and accuracy of trigonometric-leveling measurements. The surveyor's experience and skill level are also more important for trigonometric-leveling surveys as compared to differential-leveling surveys because the operator has more opportunities to affect the quality of measurements. Surveyors can introduce random uncertainty from pointing, reading, leveling, centering, and instrument-height measurements; additionally, surveyors can make transcription errors. Differential-leveling methods are considered more accurate than trigonometric-leveling methods for determining height differences between points because the number of variables that can affect the quality of measurements is substantially lower when compared to trigonometric leveling. For this reason, a trigonometric-leveling method that reduces or eliminates sources of systematic and random error and that provides methods to test and calibrate surveying instruments is required to conduct precision surveys within the USGS and is proposed in the following sections of this manual.

\section{Current Applications of Total Station Instruments in the U.S. Geological Survey}

Historically, an engineer's transit was considered the most effective way to determine the geometry of a channel, bridge, or culvert because data collection was simple, rapid, and accurate (Benson and Dalrymple, 1967). After flooding, transits were also used to collect positional data of important features such as high-water marks that correspond to peak flood stage and cross sections of a stream channel along a reach. A "transit-stadia" survey method was used to simultaneously collect horizontal and vertical positioning data. With the advent of modern land-surveying equipment, total station instruments have become the standard for rapid and accurate three-dimensional positioning using terrestrial-based (non-Global Navigation Satellite System [GNSS]) surveying methods. Total stations are currently being used in the USGS 
to establish control networks; to transfer gage datum over long distances and beneath canopy; to measure high-water mark measurements that are used for flood and inundation modeling/mapping; to complete stream-channel longitudinal-profile surveys, cross sections, and bridge and culvert geometry surveys that are used to determine discharge using indirect methods, are used for hydraulic modeling, and are used for geomorphology and sediment studies; to complete measuring point and wellhead surveys used to reference water levels to a common datum and used to facilitate water table and potentiometric surface maps and electronic coverages; to collect bathymetric and bridge-scour measurements; to do cableway inspections to determine sag and anchor stability; to complete hydraulic control surveys; and to complete glacier terminus surveys. Total stations are also used in conjunction with GNSS to georeference and orient light detection and ranging scans and to tie GNSS surveys together over long distances and beneath canopy. Although hydrologic examples are used within this manual, the trigonometric leveling methods described herein are applicable to all scientific disciplines within the U.S. Geological Survey that are engaged in land surveying.

\section{Trigonometric-Leveling Equipment}

Having a basic understanding of land-surveying equipment operation, components, and capabilities is critical to collecting quality trigonometric-leveling data. Surveyors often need to make field adjustments or troubleshoot surveying equipment if malfunctioning equipment is detected or indicated by erroneous data. In the following section, the integral components and functionality of total stations, data collectors, prisms and reflectors, tribrachs, and tripods are described to help the surveyor better understand the equipment that is needed to obtain quality observations.

\section{Total Stations}

Hewlett Packard invented the term "total station" in 1976 to promote a new electronic precision surveying instrument that was capable of simultaneously measuring distances and angles in a single integrated instrument (Bullock and Warren, 1976; Poole and Bilen, 1976). Before the invention of the modern total station in the 1980s, an EDMI was used in conjunction with another instrument that could accurately measure horizontal and zenith angles to determine the location of an objective point. Angles would be observed using a transit or theodolite and recorded by the operator. Trigonometry was used to calculate the horizontal position of the objective point relative to the station using the measured angles and distances (Brinker and Minnick, 1995). Field measurements (angles and distances) were often entered into a scientific calculator that automatically performed trigonometric calculations to eliminate potential computational errors and to increase work efficiency. Elevations could also be calculated in a similar manner by using the zenith angle and distance measurement to precisely determine the vertical (height) difference between the instrument and the reflector, which was typically a cornercube surveying prism.

Total-station instruments are essentially electronic theodolites with an integrated EDMI used to obtain horizontal and zenith angles, and slope distances that translate to XYZ coordinates through an onboard microprocessor (USACE, 2007; Rydlund and Noll, 2017). Angles are measured electronically by an electro-optical system that reads precise digital barcodes that represent the horizontal and vertical scales on the respective glass circles of the instrument (Neilson and Pickering, 1980; USACE, 2007; Leica Geosystems, 2013). A mean angular value is detected if the electro-optical system that reads the scales on the circles has duplicate readers at diametrically opposite sides of the circle, thus eliminating angular uncertainty from eccentricity in mounting the circles on the axis, circle graduation errors, and other angular errors associated with using conventional surveying instruments (USACE, 2007). The angles (and distances) are shown on the digital display of the instrument and are recorded in internal storage or on a memory card. This reduces operator errors and mistakes associated with optical instruments such as scale interpolation and transcription blunders. These data may also be stored on a paired external data-collection device, which is described in the "Data Collectors" section.

Slope distances are measured electromagnetically by the total station with an integrated EDMI that transmits a modulated carrier signal from a light source, usually an infrared laser, to a reflector (typically a corner-cube prism) at the objective location. The reflector returns some of the light to the instrument, and that light is received by a receiver lens system that focuses the signal on a photo detector for processing (Rüeger, 1990). The phase difference (or phase shift) between the transmitted signal and the reflected signal is detected by the instrument and represents the distance to the location of the target reflector (McMillen, 1993; Trimble, Inc., 2017). Some EDMIs can also measure slope distances by precisely determining the two-way travel time (time of flight) of the transmitted signal. Essentially, the measured travel time is divided in half and multiplied by the speed of light to determine the distance between the reflector and the EDMI. A pulsed laser is typically used for time-of-flight measurements, which require less energy and have a greater range but are historically less accurate than phase comparison measurements.

Total station EDMIs that use infrared or visible light as the radiation source are electro-optical instruments, whereas, EDMIs that use radio signals are considered microwave instruments. Microwave instruments may perform better than electro-optical instruments in haze and dense fog and at longer measurement distances because they transmit carrier signals with longer wavelengths; however, microwave EDMIs have a constant and distance-weighted uncertainty of about 15 millimeters $(\mathrm{mm})$ plus 5 millimeters per kilometer (Ogundare, 2016). Using a microwave EDMI has become rare 
in the surveying community because uncertainty specifications are inferior to electro-optical instruments. For example, typical microwave EDMIs have a constant error of $15 \mathrm{~mm}$ as compared to the constant error for infrared EDMIs, which is typically less than $5 \mathrm{~mm}$.

The range of the integrated EDMI for modern total stations with a single-prism assembly is up to 2,500 meters (m; $8,202 \mathrm{ft}$ ), and, with a triple prism or by operating in long-range mode with a single prism, more than $5,500 \mathrm{~m}(18,044 \mathrm{ft})$. EDMIs have a constant and distance-weighted uncertainty that is typically around $5 \mathrm{~mm}$ plus 5 parts per million (ppm) but have been reported to less than $2 \mathrm{~mm}$ plus $2 \mathrm{ppm}$, respectively, which are added together for the total EDM uncertainty. The distance-weighted error is proportional to the measured distance but can usually be minimized if observations are less than $500 \mathrm{ft}$ to reduce atmospheric and meteorological effects such as curvature and refraction as well as changes in the refractive index of air, which affects the speed of transmission of the electromagnetic radiation. EDMIs should be periodically tested on calibrated baselines to ensure they are operating to the manufacturer's specification. Procedures for testing EDM uncertainty and applying corrections is described in the "Preparing for Trigonometric Leveling" section.

Single-axis compensators that compensate for the tilt of the vertical axis of the instrument were standard on most theodolites and early total stations before the invention of the modern total station. The (vertical) compensation axis is aligned to detect tilt in the direction of the telescope, which is used to correct the zenith angle when the instrument is not perfectly level (Cothrun, 1995). Modern total stations are equipped with another compensation axis that is perpendicular to the sighting direction of the telescope, sometimes called the "transverse direction." These dual-axis compensators correct both the horizontal and zenith angles when the instrument is not completely level and when the telescope is not aligned with the horizontal plane of the total station. The optical qualities of the telescope are also important because they reduce pointing errors committed by the operator (Ogundare, 2016). The magnification of a typical surveying scope is $\times$ 30; however, some manufacturers produce an interchangeable eyepiece that can increase magnification to $\times 59$. Automatic target recognition (ATR) technology is also available on many modern total stations, which helps the operator detect and precisely point the telescope at the objective point. Field of view, minimum focusing distance, and focusing error are also important telescope attributes that should be specified by the total station manufacturer.

Another feature of the modern total station is integrated microprocessors, or internal data processing and collection devices, which have software programs that convert distance and angle measurements to XYZ coordinates; compute horizontal and vertical distances and offsets; average F1 and F2 observations (discussed in the "Trigonometric Leveling" section); perform area and volume calculations; and can correct for atmospheric conditions like temperature, pressure, and humidity (USACE, 2007; Leica Geosystems, 2013). Some microprocessors have additional functionality like coordinate geometry programs that can perform surveying calculations for resection, inverse, rotation, and translation; and can facilitate "stake-out" programs, which are the industry standard for accurately determining locations that were previously surveyed or computed. Angular uncertainty resulting from misalignment of the instrument axes (collimation error) can also be determined and stored and is automatically applied to angular observations by the microprocessor to reduce observation error (Leica Geosystems, 2013). Prism offsets (discussed in the "Prisms and Reflectors" section) can also be programmed into the total station to account for time delay of the reflected signal.

The total station can operate without a prism in "prismless" mode (sometimes incorrectly identified by the manufacturer as "reflectorless") by transmitting a signal (typically red or infrared laser) that is returned to the total station from a prismless target or feature (USACE, 2007). Prismless observations are used for estimating the position of features in remote locations that are not accessible or cannot be physically occupied, or in locations that pose a potential safety hazard for the survey crew. Manufacturers of modern total stations operating in prismless mode have reported measurement uncertainty as low as plus or minus ( \pm ) $2 \mathrm{~mm}$ plus $2 \mathrm{ppm}$; however, at longer distances the uncertainty may increase to greater than $\pm 10 \mathrm{~mm}$ plus $10 \mathrm{ppm}$ (Leica Geosystems, 2017c; Trimble, Inc., 2017; Topcon, 2018). Prismless mode should be used with caution to estimate the location of an objective point because results can be faulty if the instrument is not pointed correctly or if other reflectors are near the objective point, which may return an erroneous signal. Elevations that are obtained in prismless mode can be highly inaccurate, so the user is cautioned to use these data appropriately. Regardless of whether the total station is used in conventional or prismless mode, precise centering over the survey marker is critical for quality measurements. Precise centering is ensured by an experienced surveyor operating a precisely adjusted optical plummet.

Optical plummets, which are folded telescopes used to view the point on the ground (survey marker), are used instead of conventional plumb bobs to center the total station over the point (Brinker and Minnick, 1995). The need for plumb bobs is eliminated because the optical plummet can be used for coarse and fine centering of the surveying instrument over the ground point. Many total stations now use visible laser plummets in lieu of the optical plummet to accomplish the same objective of centering. Total station optical and laser plummets must be periodically checked and precisely adjusted, which is described in the "Preparing for Trigonometric Leveling" section.

Total-station instruments can be categorized by the angular uncertainty of the instrument, expressed in arc-seconds. Angular uncertainties less than 5" are generally referred to as "survey grade," whereas those greater than 5" are referred to as "construction grade" (Rydlund and Noll, 2017). In theory, linear (radial) error resulting from angular uncertainty can be 
calculated by taking the sine of the angular uncertainty (in degrees) of the total station multiplied by the foresight distance. For example, if a $5^{\prime \prime}$ total station is used to measure to an objective point $500 \mathrm{ft}$ from the instrument, one can expect an error of $\pm 0.012 \mathrm{ft}$ in the direction perpendicular to the line of sight (Kavanagh, 2004).

$$
\begin{gathered}
U_{L}=\sin (\Delta \phi \div 3,600) \times d_{f}, \\
U_{L}=\sin (5 \div 3,600) \times 500 \\
U_{L}= \pm 0.012 \mathrm{ft}
\end{gathered}
$$

where

$U_{L} \quad$ is the plus or minus linear error, in feet;

$\Delta \phi \quad$ is the angular uncertainty, in arc-seconds; and

$d_{f} \quad$ is the foresight distance, in feet.

Because total stations can range in angular accuracy from 30 " to models that can resolve angles to less than 0.1 " in optimal conditions, the linear (radial) error resulting from a total station of indicated angular uncertainty varies with measurement distance (table 1; Ogundare, 2016). Project requirements, budget considerations, and equipment specifications should be identified before procuring a total station; table 1 lists the estimated costs of total station instruments of varying angular uncertainty. Methods to identify, account, and correct for other sources of error, which comprise the error budget for the total station, including error associated with the integrated EDMI that are constant (independent of measured distance) and distance weighted (proportional to measured distance),

Table 1. Linear (radial) error caused by angular uncertainty of the total station at measurement distances of 200,500 , and

\begin{tabular}{|c|c|c|c|c|}
\hline \multirow{2}{*}{$\begin{array}{l}\text { Angular uncer- } \\
\text { tainty }{ }^{1} \text { of the } \\
\text { total station, } \\
\text { in arc-seconds }\end{array}$} & \multicolumn{3}{|c|}{$\begin{array}{l}\text { Linear error for the foresight } \\
\text { distance, } \\
\text { in plus or minus feet }\end{array}$} & \multirow{2}{*}{$\begin{array}{c}\text { Estimated cost } \\
\text { of the total } \\
\text { station, } \\
\text { in dollars }{ }^{2}\end{array}$} \\
\hline & 200 feet & 500 feet & 1,000 feet & \\
\hline 30 & 0.0290 & 0.0726 & 0.1452 & - \\
\hline 20 & 0.0193 & 0.0484 & 0.0967 & - \\
\hline 10 & 0.0097 & 0.0241 & 0.0483 & $3,000-7,000$ \\
\hline 5 & 0.0048 & 0.012 & 0.024 & $5,000-13,000$ \\
\hline 1 & 0.0009 & 0.0023 & 0.0046 & $312,000-40,000$ \\
\hline 0.5 & 0.0004 & 0.0011 & 0.0022 & $25,000-35,000$ \\
\hline 0.1 & 0.0001 & 0.0001 & 0.0003 & - \\
\hline
\end{tabular}
1,000 feet along a horizontal line of sight.

$[-$, no data $]$

${ }^{1}$ The angular uncertainty is determined by the manufacturer specified from industry-recognized standards that may require multiple sets of observations in the direct and reverse instrument orientations.

${ }^{2}$ Quotes are from various sources for various brands of total stations. Estimated costs are based on U.S. dollars in 2020.

3This cost range is for a multistation with an integrated global navigation satellite system or other similar equipment. are described in the "Preparing for Trigonometric Leveling" section. Field operation of the total station is discussed in the "Trigonometric Leveling" section.

\section{Robotic Total Stations}

The robotic total station functions much like a conventional total station except the operator commands the robotic total station remotely from the objective location by transmitting instructions using a radio signal (Noll and Chu, 2017). Instructions are transmitted from a radio that is connected to a data-collection device and are received by a second radio that is connected to the total station. Robotic total stations use electromagnetic, optical, or both sensing technologies and use advanced algorithms to detect, track, and precisely point the instrument at the center of the target (Leica Geosystems, 2015). This technology is called ATR and can help reduce measurement uncertainty from pointing errors. Once the target has been detected, a paired internal motor rotates the alidade and telescope (trunnion axis) to maintain a lock on the target (typically an omnidirectional prism). The operator commands the total station to make a measurement when the instrument is locked on the prism. After the measurement is made, and the data have been collected, the operator moves to the next objective point and continues the land survey.

The omnidirectional, or 360-degree $\left(^{\circ}\right)$ corner-cube prism, described in the "Prisms and Reflectors" section below, is the preferred reflector for robotic total-station surveys because the prism does not have to be constantly aligned with the direction of the transmitted signal, nor does the configuration compromise the assurance of the target plumb line. The main benefit of the robotic total station is that surveys can be completed by a single person instead of a multiperson survey crew. Additionally, measurement procedures are automated and standardized with robotic total stations, which prevents pointing errors that may occur by instrument operators who can be affected by variables such as fatigue and adverse weather conditions.

In the industry, surveying firms have reported a threefold increase in productivity for projects that required substantial terrain measurements and were conducted by a one-person survey crew, as opposed to the traditional two- or three-person crew (Leica Geosystems, 2017a). The cost savings, combined with increased work efficiency and improved measurement precision, make the robotic total station a compelling alternative to conventional total stations for many land-surveying firms; however, anecdotal evidence suggests that many surveying firms may wait to purchase the instrument until the cost of the technology decreases. Additionally, many firms will send out a second surveyor during robotic surveys to ensure the instrument is operating properly, especially in adverse weather conditions or in highly wooded or highly urbanized areas, where the optical, electromagnetic, or both systems may have difficulty detecting and tracking the prism. Ultimately, procurement of robotic total stations should be based on project requirements, budget considerations, and the land-surveying 
experience of the staff. Practice indicates that an experienced two-person survey crew (crew chief/rodman and instrument operator) is invaluable and may outperform a one-person crew with a robotic total station for various field conditions.

\section{Total Station Maintenance}

Because total stations are frequently used in adverse environmental conditions, they require routine inspection (typically before and after field campaigns) by the survey team to ensure contamination like dirt and water do not affect the proper operation of the instrument or measurement quality. The exterior of the total station should be cleaned regularly according to the manufacturer specifications because dirt and dust can accumulate on surfaces that may affect the movement of the instrument (California Department of Transportation, 2015). Surfaces, other than lenses and other optical components, can generally be cleaned with a mild household cleaner (glass and surface cleaner) with cotton swabs, pipe cleaners, or other nonabrasive fabrics (Brinker and Minnick, 1995). The external surface of the optical lenses should be cleaned with optical-quality glass cleaner and a fine lens brush, lens tissue, or camel hair brush so that the lens and the lens coating are not scratched-never use silicone-treated tissues to clean external optical glass surfaces because it may damage coated lenses (California Department of Transportation, 2015). The interior of the total station and optical lenses should never be accessed or cleaned except by the vendor or manufacturer during periodic servicing in a controlled environment such as a laboratory or sterile warehouse. Clamp screws, tangent screws, leveling screws, and exposed metal joints should be carefully inspected because they can affect the horizontal and vertical movement of the total station (Brinker and Minnick, 1995). These components should operate smoothly and should not be treated with lubricants such as oil or grease because they can attract dust and grit, which accelerates wear, and can potentially damage parts and affect measurement quality. If the total station gets wet during operation, it should be unpacked from the case (along with other accessories that were exposed), wiped dry using the previously described methods, remembering to treat the glass surfaces as described above, and left outside of the case in a secure location to ensure adequate drying. To prevent corrosion or propagation thereof, do not store wet instruments inside the instrument case, and do not access internal components that appear to be wet.

Total stations should be regularly inspected for damage before and after surveying campaigns and should be stored in a safe and secure location in the protective carrying case between surveys. Damage to the total station should be documented, and a repair quote should be obtained from the manufacturer or vendor. Other users of the instrument should be made aware that the total station is damaged and should not be used for data-collection activities until it is serviced and repaired.
In addition to routine cleaning and damage inspection, total station instruments also require annual manufacturer (or vendor) service calibrations that address issues such as alignment of the EDMI and collimation adjustments as part of testing to ensure product specifications for angular uncertainty and distance (Rydlund and Noll, 2017). Total stations should be sent to the manufacturer or vendor for calibration periodically based on time, frequency of use, or both. Indicators of poor measurement quality potentially attributable to neglect of cleaning and maintenance are described in the "Preparing for Trigonometric Leveling" section.

\section{Data Collectors}

Data collectors, commonly referred to as electronic field books, are handheld electronic information devices that were designed to automate data recording and processing of field observations by interfacing with a total station during land surveys (Brinker and Minnick, 1995). Angles and distances are automatically recorded in the data-collection device and are converted to XYZ coordinates that reference an assumed or known coordinate system. Before the advent of electronic data collectors in the 1990s, angle and distance measurements were manually recorded in a field book and then plotted by hand when the surveyor returned to the office (USACE, 2007). By automating this process, electronic data collectors have increased work efficiency and have reduced reading and transcription mistakes. Furthermore, data collectors perform automated quality-assurance checks that help the operator identify blunders and collect reliable data. Data collectors can interface with personal computers for the purpose of transferring field data for postprocessing and map production.

Modern data-collection devices have increased functionality, processing power, memory, and data storage capacity. These devices have enough storage capacity to record and process field observations, collect metadata, and store large electronic files. Essentially, these devices are handheld personal computers that have all the functionality of integrated microprocessors (described in the "Total Stations" section) and an office computer. Many tasks that were typically done in the office on personal computers, such as drafting, plotting, and design, can now be done in the field using large liquid crystal display screens and a graphical user interface (USACE, 2007). Many units are equipped with an advanced operating system and land-surveying software that can perform a variety of complex computations such as least-squares and compassrule adjustments of control networks, horizontal and vertical datum transformations, and level net adjustments (USACE, 2007; Carlson, 2017).

Modern data collectors have the capability to access the internet and can also wirelessly interface with a variety of advanced surveying instruments like robotic total stations, GNSS receivers, digital levels, and laser scanners. Data collectors are lightweight and shockproof, can resist water and dust, and have lithium-ion batteries that can operate in cold weather 
for extended periods of time without charge. Some high-end models are also equipped with a GNSS receiver and a highresolution camera that can georeference photographs and overlay satellite imagery on the liquid crystal display screen. Field data can be downloaded wirelessly from the data collector to an office computer to be edited, analyzed, and further postprocessed. Various programs can be used, such as a computeraided design program, coordinate geometry, or geographic information system, for postprocessing, adjustments, and map production. Because of the increased memory and storage capacity, geographic information system and computer-aided design files can also be uploaded to the data-collection device for use during survey campaigns.

External data collectors are mobile, versatile, and have the functionality of integrated microprocessors (or internal data processing and collection devices) and may be more user friendly. As previously described, the functionality, storage capacity, and processing power of the modern external data-collection device may be greater than that of the integrated microprocessor, but it can be expensive. A cost-benefit analysis should be performed based on project requirements, surveying needs, and budget limitations before procurement of an external data-collection device to determine potential return on investment. For example, if a total station is used solely as a leveling instrument, an external data-collection device may not be needed; however, because some internal data-collection devices and processors (typically older units) do not collect and store metadata like electronic field notes and sketches, the operator must make detailed records during the survey campaign in case of a blunder, to verify field observations, or both. Detailed, manual (hand-written) notes take time to record and can be damaged or lost because of adverse site conditions like inclement weather. The quality and content of manual notes also vary from surveyor to surveyor, which may create additional work for the analyst who is interpreting these data. If the surveyor makes a transcription blunder or measurement error that is not accounted for in the manual notes, the survey crew may need to return to the field site, which may be a remote location requiring substantial travel time, to re-survey a section or re-run all the original survey lines. Electronic metadata provides the analyst with the ability to troubleshoot apparent erroneous measurements or blunders in the office without having to redeploy the survey crew. The external data collector may serve as an electronic field book and a backup to manual field notes and thus is a valuable resource to the surveyor for collecting, processing, archiving, and quality assuring data from surveying.

Although external data-collection devices are an invaluable tool for the surveyor to collect quality measurements, the additional cost to purchase and maintain these instruments may be prohibitive. Service contracts may need to be purchased to update operating systems and software packages, upgrade firmware, or replace malfunctioning or damaged hardware. Integrated Bluetooth antennas and data-collector cables are notoriously fragile and are expensive to replace. Sometimes total stations are not compatible, or functionality is severely reduced, when paired with less expensive but comparable models of data collectors from other manufacturers. It is important for the purchaser to at least obtain a certificate from the manufacturer certifying that the total station, data collector, and other surveying instruments meet cross-compatibility requirements with models from other manufacturers as specified by the purchaser. External data-collection devices should be regularly serviced and cleaned, stored, and maintained according to the manufacturer's specification.

Recent innovations by instrument manufacturers have completely integrated the external data-collection device into the total station. These "multistations" or "smart total stations" have all the functionality of external data-collection devices including large screens, a full video graphic display, an alphanumeric keyboard, an advanced operating system, onboard land surveying software, and some with an integrated surveygrade GNSS receiver and laser scanner. Although these instruments are expensive and may be cost-prohibitive, surveyors should consider the advantages of having a fully integrated surveying system in a single instrument.

\section{Prisms and Reflectors}

Retroreflectors, also identified as corner-cube prisms or retroprisms, are used to determine the slope distance from the total station (intersection of the vertical axis with the sighting axis of the EDMI) to the target location by reflecting the electromagnetic signal that is transmitted by the integrated EDMI. The reflected (returned) signal is focused on the photo detector by the EDMI receiver lens system, and a distance is determined using a phase comparison (phase shift) method described in the "Total Stations" section (Rüeger, 1990; McMillen, 1993). A corner-cube prism is used as the reflector because the reflected beam (signal) is always parallel to the incident beam (transmitted signal) even when the incident beam is not parallel to the optical axis and is not perpendicular to the surface (face) of the reflector (fig. 2). This is an important and intrinsic attribute of the corner-cube prism because it is nearly impossible to point a prism (or any reflector) at a transmission source (EDMI) so that its incident face is perfectly normal to the path of the incident beam, so the transmitted signal always approaches the surface of the reflector at a non-normal angle.

Corner-cube prisms are constructed from a solid piece of cylindrical optical glass, which has a circular planar surface that faces the EDMI and is aligned such that the glass face is approximately perpendicular to the transmitted signal (McMillen, 1993). The optical axis of the prism is orthogonal to the planar surface (face) of the optical glass that faces the EDMI (fig. 2). Three facets (surfaces) are constructed perpendicular to one another in the back of the prism to form a pyramid shape that has an apex (center) along the optical axis, opposite the face. The angle of the facets relative to the optical axis are equal and are greater than the critical angle of the prism face, which ensures the transmitted signal is internally reflected. 
$\boldsymbol{A}$

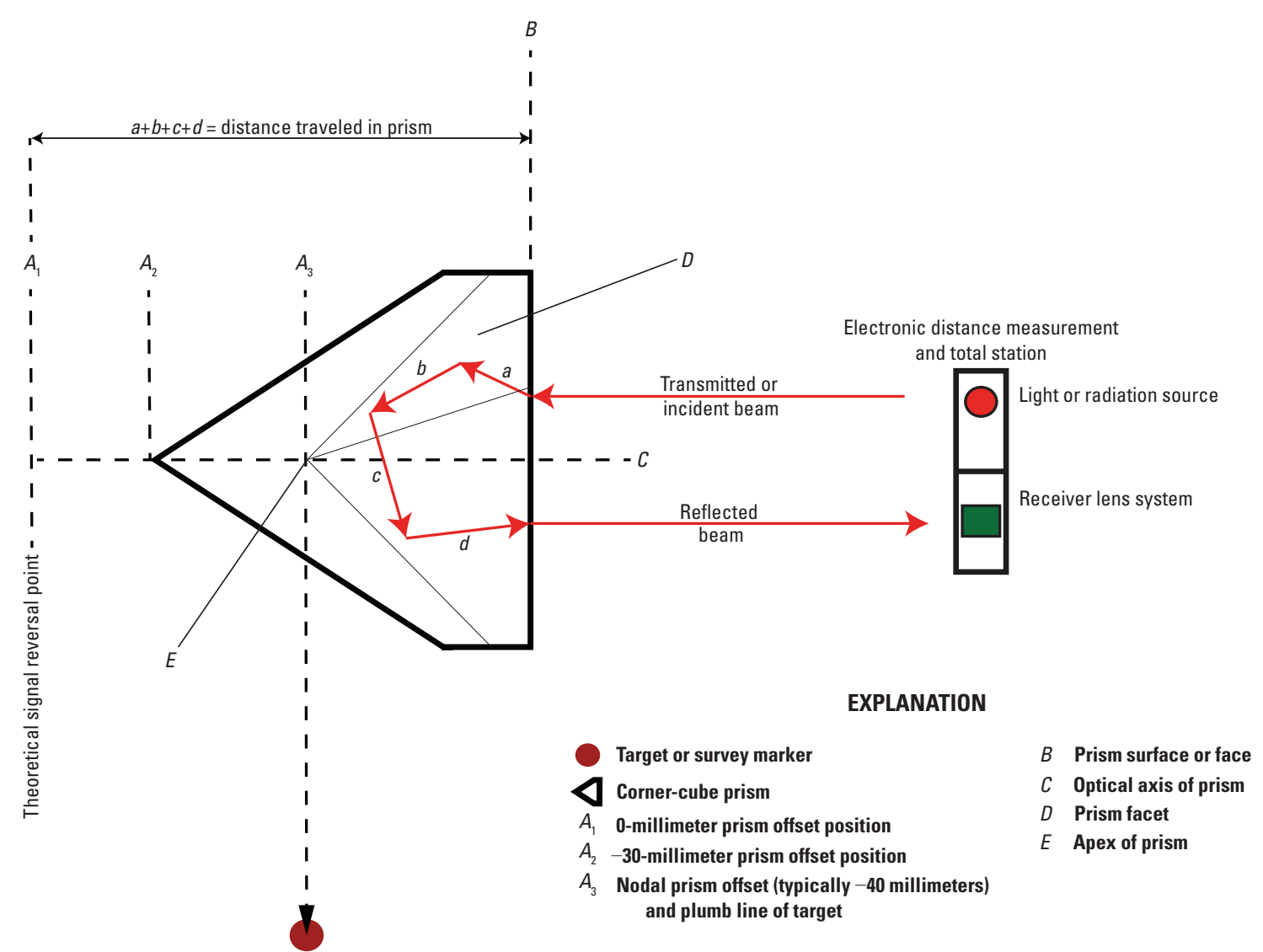

B

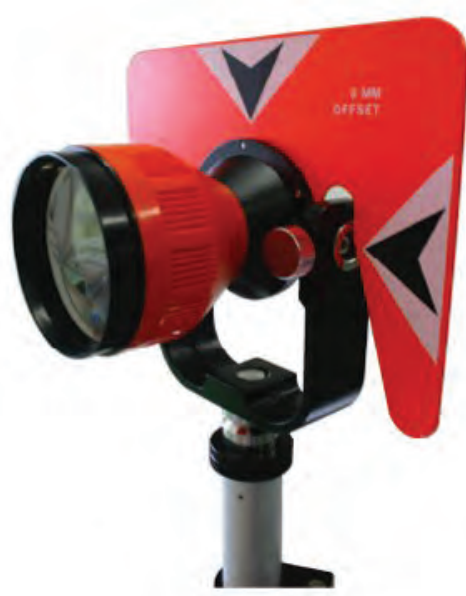

0-millimeter offset prism assembly -30-millimeter offset prism assembly

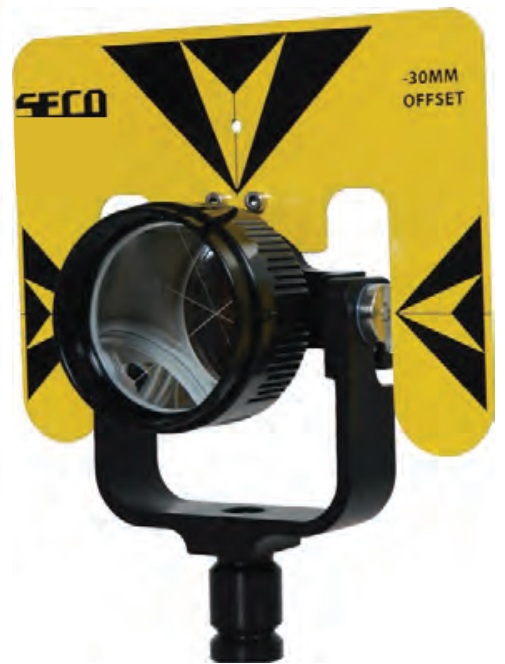

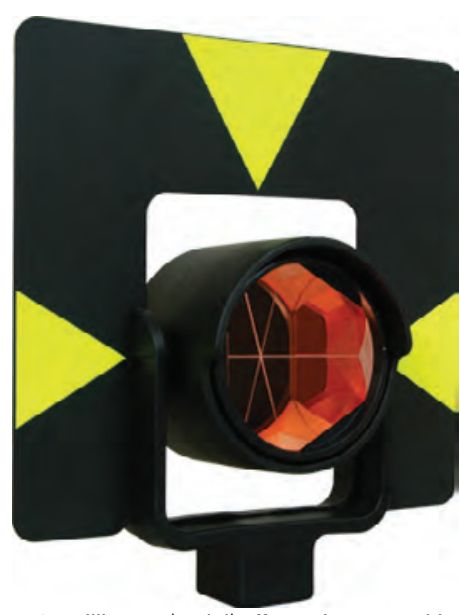

-34-millimeter (nodal) offset prism assembly

Figure 2. Prism equipment used for surveying. $A$, axes, incident beam, reflected beam, angle of incidence, nodal center, and other component parts of a corner-cube prism; $B$, corner-cube prism assemblies with various prism offsets; $C$, various types of prisms and prism accessories; and $D$, some of the components of a prism assembly. Photographs and diagrams (in $B, C$, and $D$ ) are from SECO Manufacturing Company; used with permission. 

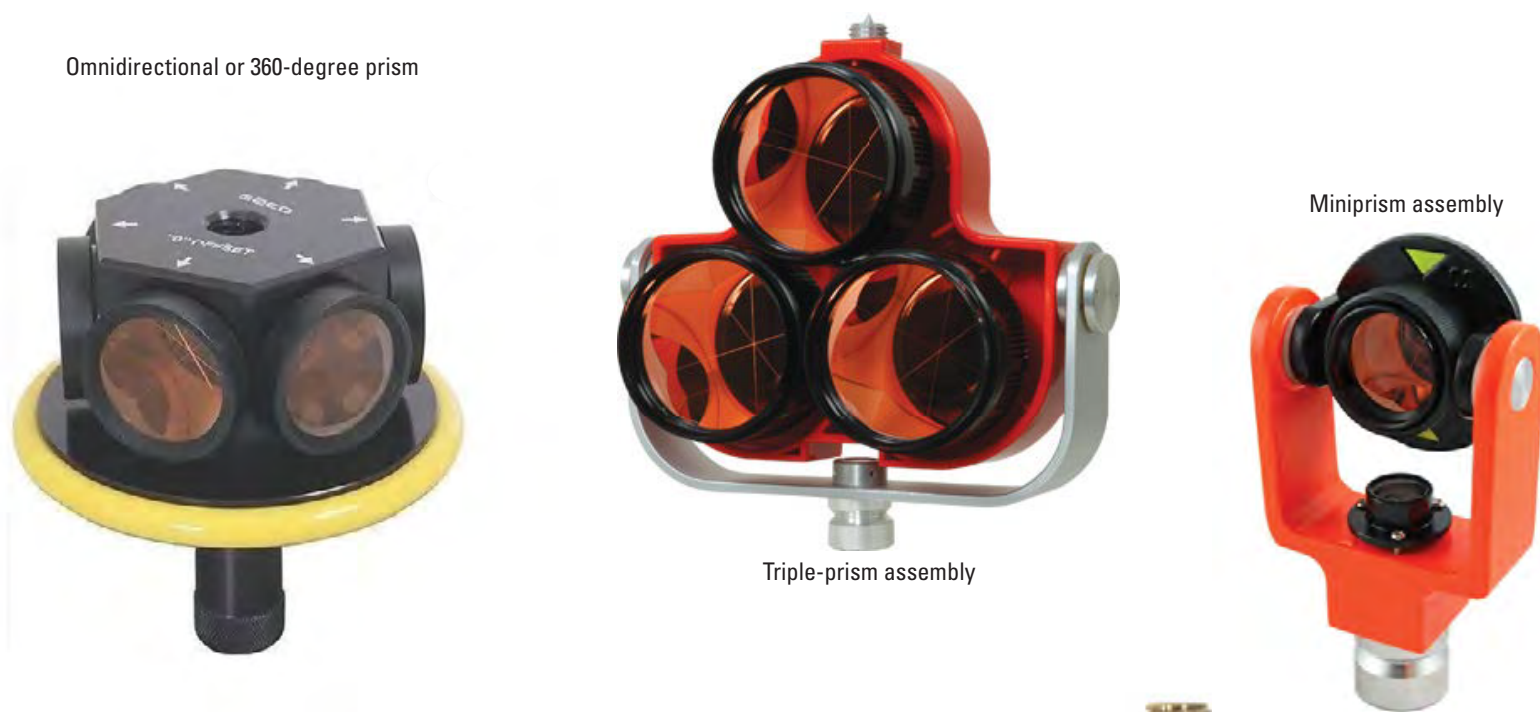

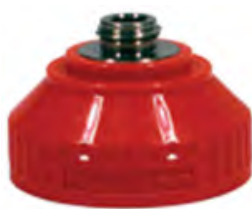

-40-millimeter and -30-millimeter prism adapter

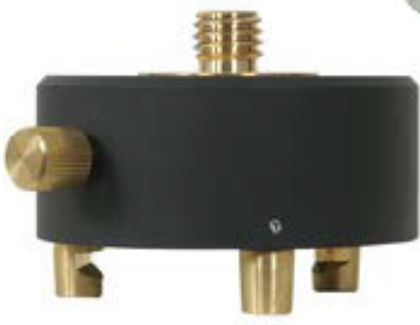

Tribrach adapter

D

Corner-cube prism cap

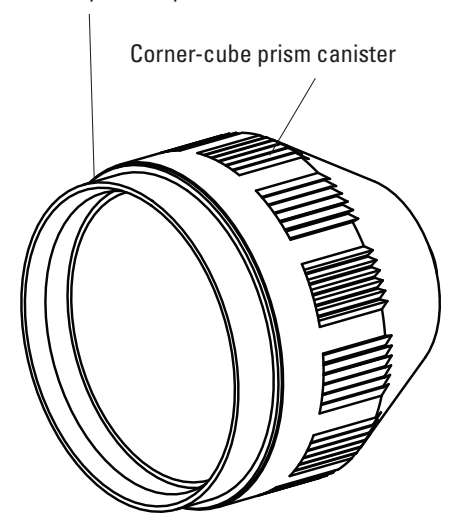

Corner-cube prism
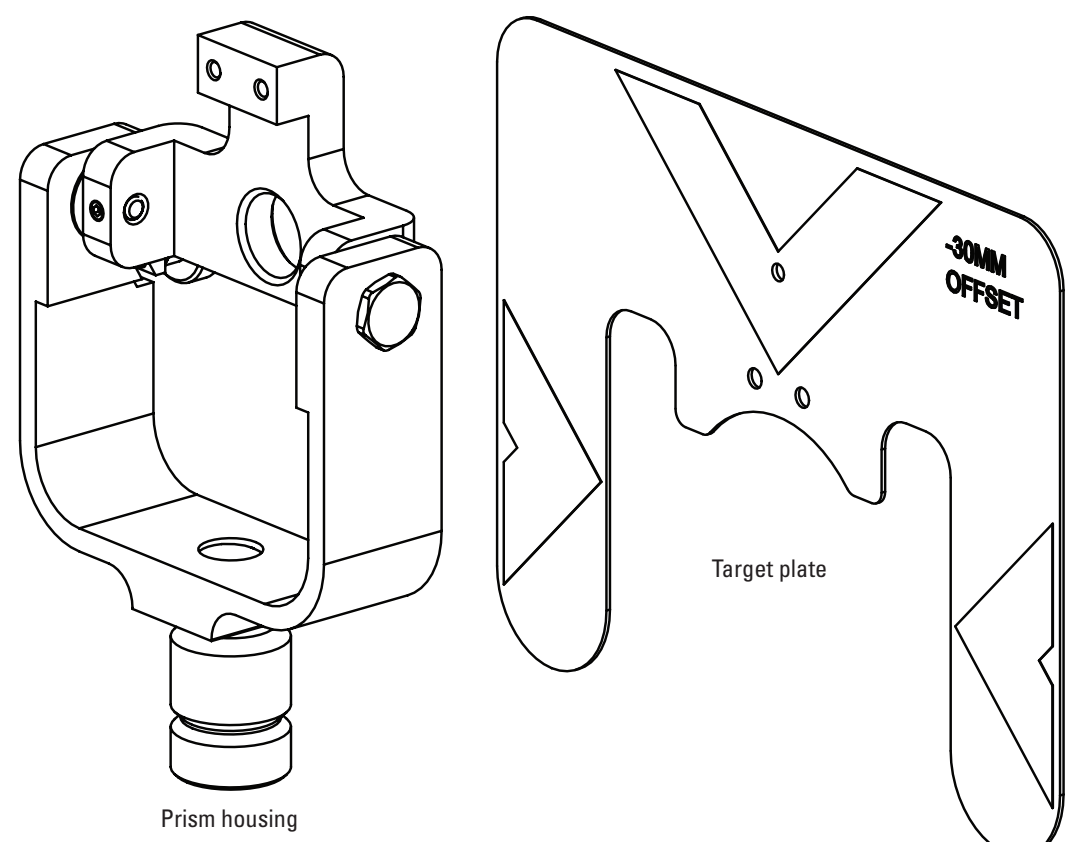

Figure 2. Prism equipment used for surveying. $A$, axes, incident beam, reflected beam, angle of incidence, nodal center, and other component parts of a corner-cube prism; $B$, corner-cube prism assemblies with various prism offsets; $C$, various types of prisms and prism accessories; and $D$, some of the components of a prism assembly. Photographs and diagrams (in $B, C$, and $D$ ) are from SECO Manufacturing Company; used with permission. 
The geometry of a corner-cube prism is best visualized by imagining a rectangular cube of glass from which the prism is cut by making a diagonal cut that removes a corner. The surface created by the diagonal cut is the face that is pointed at the EDMI.

The transmitted signal reflects from each facet and is returned to the EDMI through the face (or surface) of the optical glass along a path that is parallel to the transmitted signal. The corner-cube prism is housed in a protective canister that can be mounted to a tribrach (discussed in the "Tribrachs" section), which is secured to a tripod or a prism pole that may or may not be stabilized by a bipod to ensure the prism is stable and plumb above the survey marker. The components of a prism assembly differ among manufacturers but may consist of a corner-cube prism with canister and cap, a prism housing that can be tilted and reversed, and a target plate; in some cases, the assembly may also contain a prism-offset adapter, a tribrach, and a tribrach adapter (fig. 2).

Because the velocity of the transmitted signal (typically infrared light) is relatively slower in the optical glass as compared to air, the refractive index of the optical glass is used to correct for the signal delay (McMillen, 1993). Typical prisms have a refractive index of 1.509 , which is multiplied by the traveled distance of the transmitted signal in the optical glass to get the equivalent distance the signal would have traveled in the same amount of time in the air (McMillen, 1993). This equivalent distance is called the prism offset (or prism-offset correction), which is indicated by the position of the prism apex relative to the plumb line of the survey marker (fig. 2) and differs with the brand and model of the prism. Some manufacturers of conventional corner-cube prisms account for this correction by constructing the apex of the prism a distance away from the plumb line (of the survey marker) that is equivalent to the prism offset (labeled as $A_{1}$ on fig. 2). This type of prism is called a zero-offset prism and does not require a correction for the horizontal offset caused by the additional equivalent air distance traveled by the signal. However, the distance and angle observations to this type of prism can have small errors unless the prism assembly includes a target that enables the surveyor to place the horizontal and vertical crosshairs on the correct location for the intersection of the vertical line through the ground point and the rotational axis of the prism.

To ensure the instrument is pointed correctly, manufacturers of corner-cube prisms may align the apex (center) of the prism with the plumb line of the survey marker, which is called a nodal alignment (or nodal position; labeled as $A_{3}$ on fig. 2). The operator can verify a prism is in the nodal position by focusing the crosshairs of the instrument on the apex of the prism at close range (less than $5 \mathrm{ft}$ ) and then by having the rod operator tilt and rotate the reflector. If the crosshairs remain pointed at the center of the prism, it is in nodal position; if the crosshairs are no longer aligned with the apex of the prism, the prism is in a position other than nodal. A nodal alignment is particularly important for targets near the minimum operating range of the EDMI (or total station) if the instrument operator uses the apex of the prism as a pointing target for horizontal and zenith angle observations because pointing error over short measurement distances can become a significant part of the error budget (Berntsen, 2017). At longer measurement distances, pointing error from misalignment of the prism apex and the plumb line of the survey marker caused by non-nodal alignment of the prism becomes insignificant. However, there is no rule of thumb because the error generated needs to be contrasted with the allowable error budget for the survey. Nodal offsets are typically specified by the manufacturer and are physically identified on the prism housing or assembly. Prism offsets other than nodal are constructed by manufacturers to reduce the effects of large incident angles that can occur if the prism is not pointed at the total station or EDMI; some prism offsets are designed to correct for the misalignment of the plumb line of the survey marker through an EDMI and the position of the photodiode in the EDMI (Cothrun, 1994); and some manufacturers simply have different opinions regarding the optimal prism-offset correction (McMillen, 1993).

The prism-offset correction is usually physically identified by the manufacturer in metric units on the prism or prism assembly and should be programmed into the data-collection device (integrated or external) before a survey campaign to ensure the correct offset is applied to the distance measurement (Berntsen, 2017). Typical prism offsets are $0 \mathrm{~mm}$, $-17.5 \mathrm{~mm}(-0.0574 \mathrm{ft}),-30 \mathrm{~mm}(-0.0984 \mathrm{ft}),-34 \mathrm{~mm}$ $(-0.1115 \mathrm{ft})$, and $-40 \mathrm{~mm}(-0.1312 \mathrm{ft})$. For these examples, the prism offset is negative because the offset value must be subtracted from the measured EDM distance to account for the additional travel time of the transmitted signal inside the prism. Prism offsets can also be positive if the apex of the prism is substantially offset from the plumb line towards the direction of the total station. Some prisms come with adapters that allow the operator to adjust the prism offset so that it can be used in conjunction with other prisms of the same offset. Similarly, some manufacturers construct prism housings with two different offsets - the first offset is for direct orientation of the prism housing, and the second offset is for reverse orientation of the prism housing. This dual-offset feature allows the surveyor to efficiently switch between prism offsets by unscrewing the prism from the prism housing, reversing the housing (about $180^{\circ}$ rotation around tilting axis), and then screwing the prism back into the side of the prism housing that is currently facing the EDMI or total station. Typical offsets for direct and reverse prism housing orientation are -30 and $0 \mathrm{~mm}$, respectively (Berntsen, 2017).

Using zero-offset prisms is not recommended because they exacerbate many of the errors that nodal prisms reduce such as pointing error at short distances (McMillen, 1993). Combining prisms with prism assemblies and housings from different manufactures is not advised because prism offsets are variable and are determined by the manufacturer (McMillen, 1993). Combining two or more brands or models of prisms and prism housings can result in an unknown prism-offset correction. Applying an incorrect prism offset is a systematic 
error that will result in erroneous data; random error from a damaged retroreflector or an inferior design can also cause an erroneous measurement.

Retroreflectors must be constructed of quality optical glass because microscopic imperfections along the corner angles and surfaces (facets) of the prism can affect the accuracy of the distance measurement (Nindl and Mao, 2010). Inferior optical manufacturing processes can compound the effects of inferior glass causing undesirable divergence of the reflected beam, which then reduces the signal intensity as it approaches the EDMI for processing. In some cases, with lowquality or damaged optical glass, operating at the maximum range of the surveying instruments, or both, the intensity and direction of the reflected beam may be corrupted such that the measured distance cannot be resolved or may miss the EDMI completely (Berntsen, 2017). Using prisms that are chipped or cracked is not recommended. Chips are likely to reduce effective range, and cracks can change the design path of the signal and induce substantial errors in the observed distance. Beam divergence for most optical glass used for retroreflectors in the surveying industry is a few arc-seconds, so the operator must be aware of the limitations and operating range of the equipment.

The diameter of the surface of the corner-cube prism is also important because it can affect the operating range of the prism (Nindl and Mao, 2010; Berntsen, 2017). The diameter of the surface of most conventional corner-cube prisms is $62 \mathrm{~mm}(0.2034 \mathrm{ft})$ because it is the optimum diameter for signal processing of the reflected beam by the EDMI. A larger percentage of the transmitted signal can be reflected if a larger diameter reflector is used, but most of the additional reflected signal would pass by the EDMI and would not be received or processed. The operating range of the prism can be extended by mounting multiple prisms of the same brand, model, and offset together. The pointing target for a triple-prism assembly (fig. 2) is usually indicated by the tilting axis for horizontal alignment and the plumb line of the target vertical alignment. Target plates should be used with triple-prism assemblies to help the instrument operator determine the pointing target. Triple-prism assemblies should not be used for measurement distances less than $500 \mathrm{ft}$ and are not required for trigonometric-leveling campaigns except in instances when the position of a distant target needs to be measured.

Various types of corner-cube prisms are produced by manufacturers to meet specific project requirements. Peanut prisms are used to precisely determine the location of small features like cracks and corners that cannot be easily accessed and measured with larger corner-cube prisms (Berntsen, 2017). Mini-prisms can be discreetly and permanently mounted to objects like dams that may require monitoring over long periods. Omnidirectional, or $360^{\circ}$ prisms, consist of six corner-cube prisms that are used for robotic total-station surveys because they do not have to be rotated or tilted for accurate alignment with the EDMI. One of the six prism surfaces will always be pointed at the instrument, allowing the surveyor to focus more intently on instrument operation and data collection. Omnidirectional prisms can be equipped with a transmitter that broadcasts an electromagnetic signal to the robotic total station so the movement of the operator can be tracked.

Corner-cube prisms and other reflectors must be properly maintained according to manufacturer specifications to ensure measurement quality during surveying campaigns. This maintenance includes proper cleaning, storage, and periodic factory or laboratory calibrations by the manufacturer or vendor. Prism offsets should also be periodically verified by the operator to ensure the proper correction has been entered into the external or integrated data-collection device - but never in both because that will result in a double correction. Opticalglass surfaces should be cleaned with optical-glass cleaner and a nonabrasive fabric (California Department of Transportation, 2015). Prisms should be removed from tribrachs after use and stored in a dedicated compartment during transit that is padded to absorb blows or shocks during transport to prevent damage.

\section{Tribrachs}

Tribrachs are used to secure a total station, a prism assembly, or other surveying-system components to a tripod platform so that minimal adjustments are needed to center and level the instrument over a survey point such as a monument, stake, or other survey marker. The tribrach (fig. 3) is fastened to the bottom of the tripod platform by carefully tightening a central fixing screw, which is threaded through a bracket that is bolted to the tripod (U.S. Department of the Army, 1980). The surveying instrument is secured to the tribrach through three machined openings that receive the legs (pins) of the surveying instrument (Porter, 2001). The legs or pins are secured to the locking ring inside the tribrach with a lever mechanism to ensure the surveying instrument does not move. Tribrachs are equipped with an optical or laser plummet for precise alignment over the survey marker (USACE, 2007). Three leveling nuts are used to differentially adjust the tribrach so that it is level, which is ensured by observing the circular bullseye level on the tribrach during the leveling procedure. The method to level the tribrach is comparable to the procedure to adjust the plummet and level bubble; see steps 1-7 in the subsequent "Level Bubble and Plummet Adjustments" section.

Tribrachs are used for precision traversing where the survey crew "leap frogs" the surveying instruments by interchanging the prisms and total station at the backsight, foresight, and station locations (USACE, 2007). This forcedcentering method reduces potential error from instrument leveling and centering because the tribrach is never removed from the tripod; and increases work efficiency because the tripod/ tribrach assembly does not have to be recentered or releveled over the ground point between observations. The operator simply releases the locking mechanism of the tribrach, removes the instrument, and moves it to the next location; however, minor adjustments of the tribrach are typically required to 
$\boldsymbol{A}$

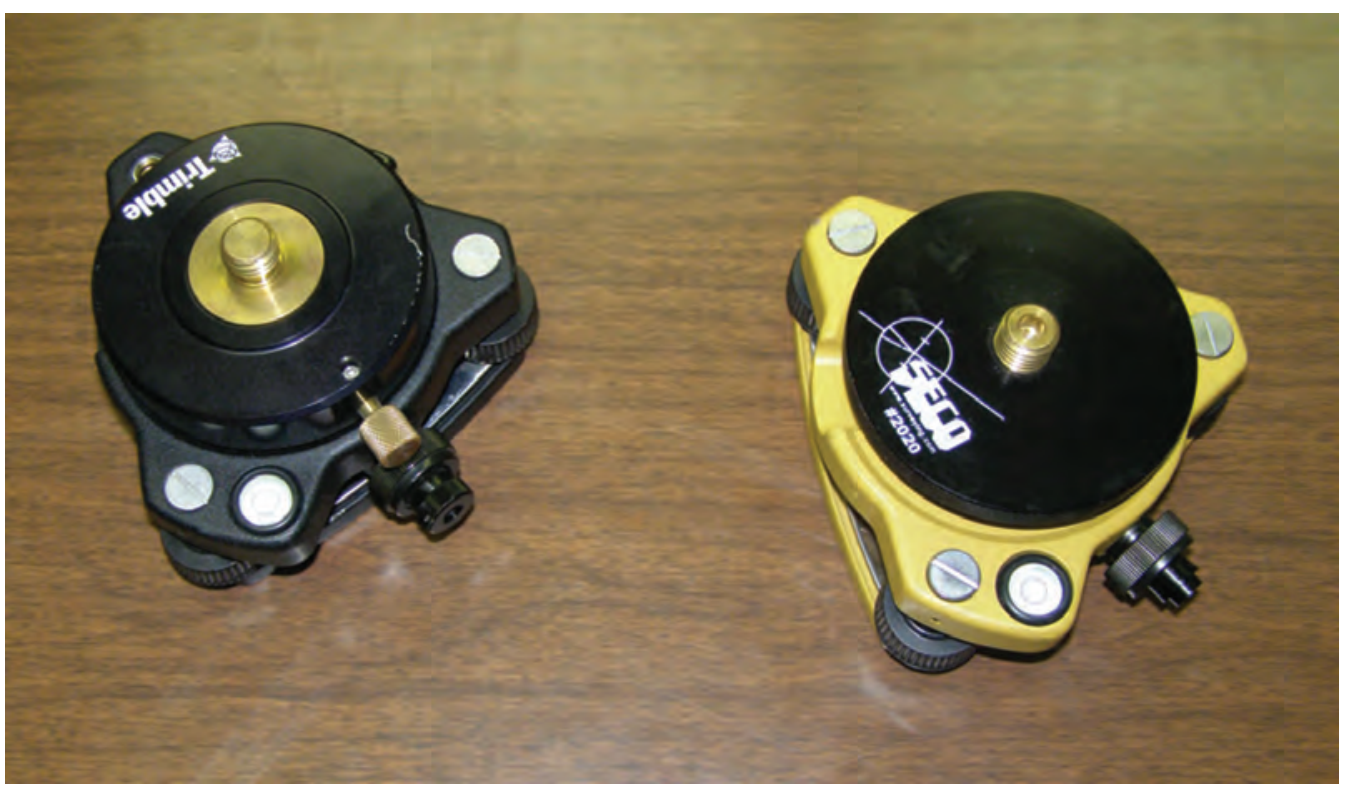

B

C
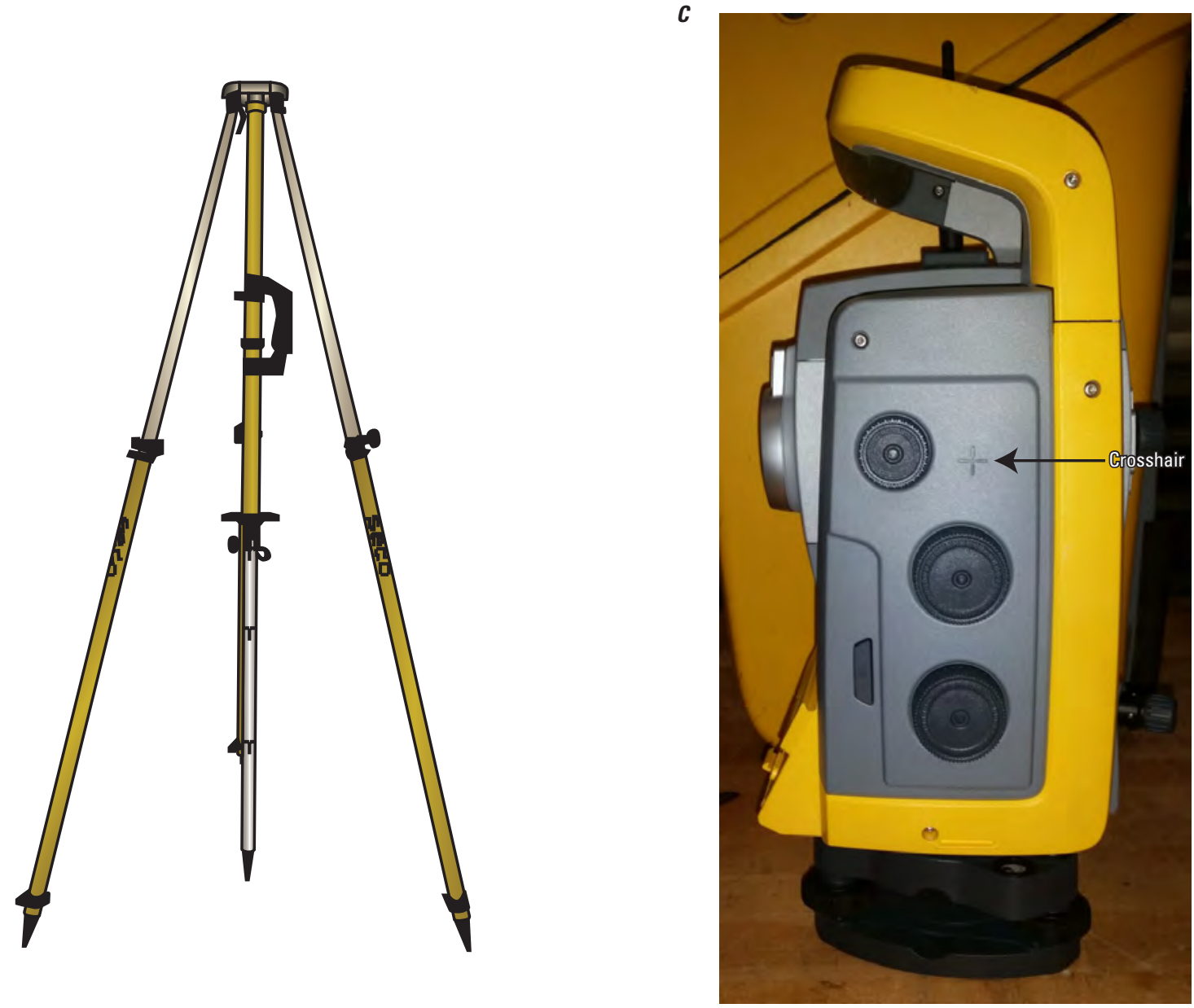

Figure 3. Trigonometric levels surveying equipment. $A$, tribrachs (photograph from Rydlund and Densmore, 2012); $B$, fixed-height tripod with adjustable center pole (photograph from SECO Manufacturing Company; used with permission); and $C$, total station with a crosshair indicating the tilting axis of the instrument. The crosshair is used as a measuring point for the instrument height. 
ensure the instrument is level and precisely centered over the survey marker. Precision alignment and leveling must be verified by the operator before subsequent observations are made.

Tribrachs are one of the most important, versatile, and often-overlooked pieces of surveying instrumentation for running quality trigonometric leveling. Tribrachs should be stored in a protective casing when they are not being used because the circular level and optical or laser plummets are sensitive to vibration and shock. For optical plummets, the focusing knob should not be overtightened because it can damage the mechanism. Do not overtighten the tribrach leveling nuts and ensure they are returned to the midrange default position after use (California Department of Transportation, 2015). The leveling nuts should also be cleaned regularly. The tribrach-leveling bubble and the optical plummet should be periodically inspected (the inspection period depends on use) to ensure proper adjustment. Methods to determine and adjust the tribrach-leveling bubble and plummet are described in the "Preparing for Trigonometric Leveling" section. In addition to recommended cleaning and operation, they must be regularly maintained according to manufacturer specifications to ensure proper operation before and after surveying campaigns.

\section{Tripods}

Tripods provide a stable platform for surveying equipment with three support legs that are positioned and anchored some distance from the vertical axis of the instrument, which is mounted on a tribrach that is secured to the surface of the platform. A central fixing screw, which is threaded through a bracket that is bolted to the bottom of the tripod platform (head or baseplate), is used to secure the tribrach (and surveying instrument) to the platform through an opening on the tripod head (U.S. Department of the Army, 1980). The feet of the tripod, which are anchored into the ground by the operator, define the corners of a hypothetical triangle with the survey marker positioned near the center. Because of the stability of the triangular alignment, tripods can resist shearing and lateral forces from high winds or passing vehicles while maintaining precise alignment (centering) and level over the survey marker (California Department of Transportation, 2015). The platforms are load bearing and can support the weight of heavier surveying instruments like a total station, GNSS receiver, or laser scanner. High torsional rigidity, or the stability (stiffness) of a piece of material when torque is applied, along with a load-bearing capacity and minimal horizontal and vertical drift are important attributes of a quality tripod (Leica Geosystems, 2017b). Tripods should be well researched before procurement to identify specifications that will ensure quality surveying measurements.

Adjustable, or adjustable-height, tripods are highly versatile and can be used with most precision surveying instruments. They can accommodate operators of various heights without moving the position of the feet and can be easily adjusted by loosening the clamps that release the extendable legs. Adjustable tripods with a dual-clamp, or double-clamp, locking mechanism are recommended because the redundant clamp ensures instrument stability and alignment (centering) over the survey marker. The clamps are easily adjusted with a wing screw, quick-clamp lever, or both, and can also be used to coarsely level the instrument.

A tripod with an adjustable center pole with fixed-height dowel stops is a compelling alternative to a conventional adjustable tripod (previously described) because (1) errors from centering and instrument-height measurements (both total station and prism) can be reduced, (2) the amount of time required to precisely center and level the surveying instrument can be reduced, (3) the need to calibrate the optical or laser plummet is eliminated because centering is forced by the adjustable center pole - not determined from the plummet, and (4) the mounting platform of the tripod may be more stable because of the 4th anchor on the ground - the three feet on the tripod legs plus the point on the adjustable center pole. The adjustable pole is centered on the tripod platform, which is supported by the three tripod legs and extends down along the plumb line to the survey marker. It is typically $2 \mathrm{~m}$ long with fixed-height dowel stops at 1.5, 1.8, and $2.0 \mathrm{~m}$ to accommodate operators of various heights. The dowel stops allow the surveyor to set a fixed instrument height (HI) and eliminate the need for height measurements (and associated uncertainty) from a fixed ruler. At the time of this writing (2020), the tripod with an adjustable center pole seems to be an emerging technology that may need additional field testing to ensure quality observations and platform stability. Many manufactures do not produce this type of tripod so currently (2020) they are not widely available and may be cost prohibitive. However, it is recommended that USGS surveyors monitor this technology as it develops because of the potential benefit of reducing measurement uncertainty from instrument heights and centering while also increasing platform stability and work efficiency. This type of tripod may be particularly useful during leap-frog surveys (discussed in the "Trigonometric Leveling" section) as the mounting platform for the prism assemblies at the backsight and foresight, and for the total station because centering and instrument-height measurements are ensured as the survey progresses to the objective location. In additional to leap-frog surveys, tripods with an adjustable center pole could be useful for any precision land survey that requires an instrument to be set up over a survey marker for a long period of time because of the increased stability from the 4th anchor point.

Tripods are constructed from a variety of materials that includes wood, fiberglass, composite, and aluminum, so quality can vary between models. Aluminum tripods are lightweight and resistant to weathering but may not provide the stability or the load-bearing capacity of wood tripods and are more prone to denting and damage, which can affect the sliding mechanism of the extendable legs (Brinker and Minnick, 1995). Wooden tripods may require more maintenance, especially as the wood sealer degrades over time and the wood becomes susceptible to swelling in wet or moist environments. Ensure tripods are stored in a dedicated compartment during 
transit because substantial damage can occur if the tripod is shuffled or bounced around when the vehicle is traveling over rough surfaces (California Department of Transportation, 2015). Maintain proper torque on all metal fittings because overtightening can destroy components and strip threads. The cap that protects the tripod platform should be used when the instrument is not attached to the tripod so that the platform is not damaged. Regardless of the tripod brand, make, or model, hardware should be routinely inspected, cleaned, and serviced according to the manufacturer's specifications.

\section{Preparing for Trigonometric Leveling}

Surveying equipment must be tested before a trigonometric-leveling campaign to ensure quality observations. These protocols include adjusting the leveling bubbles, adjusting optical and laser plummets, testing for axial defects, and testing the accuracy of the EDMI on a calibrated baseline.

\section{Level Bubble and Plummet Adjustments}

Periodic inspection of trigonometric-leveling equipment is necessary because instrument components are delicate and can be damaged during use, transit, or both. Temperature variations, humidity, and rough handling can adversely affect the adjustment of an instrument, which in turn can affect the quality of a measurement (Brinker and Minnick, 1995). Measurement uncertainty associated with damaged or poorly adjusted instruments such as prisms, tribrachs, and tripods can constitute a substantial part of the error budget for trigonometric-leveling campaigns (USACE, 2007). If damage or poor adjustment is detected during inspection, the instrument may have to be sent to the manufacturer or vendor for repair. However, some adjustments to instrument components, such as level bubbles and optical plummets, can be made by the surveyor and are described in the following section.

\section{Level Bubbles}

Tribrachs, tripods, and total stations have level bubbles that are used to determine if the surveying instrument is level over a survey marker and require routine inspection to decide if an adjustment is necessary. These components are fragile and can be forced out of proper adjustment during survey campaigns, transit, or storage, which can adversely affect the quality of measurements. Conventional types of level bubbles include cylindrical plate bubble tubes and circular (bullseye) bubbles (vials) that are built into surveying instruments. The following procedure (steps 1-10), known as the level-bubble adjustment method, can be used to calibrate the plate bubble tube for a total station, and subsequently, the circular bubble of a tribrach, which is used to secure the total station to the tripod platform (fig. 4; step 12; Brinker and Minnick, 1995). This procedure can be adopted for total stations with circular level bubbles:

1. Set up a tripod and mount the tribrach to the platform.

2. Secure the total station to the tribrach and coarsely level the instrument.

3. Rotate the instrument so the plate bubble tube is parallel to a hypothetical line connecting two of the tribrach-leveling nuts. These are labeled " $a$ " and " $b$ " on figure $4 A$.

4. Precisely center the bubble in the tube using two leveling nuts (a and b; fig. 4A). Nuts a and $\mathrm{b}$ can be turned clockwise or counterclockwise, but always in the opposite direction.

5. Rotate the instrument $90^{\circ}$ (so that the bubble tube is now perpendicular to the hypothetical line connecting nuts $a$ to b) and use the remaining, third nut (labeled "c") to precisely level the instrument (fig. 4B)

6. Repeat steps 4 and 5 until the bubble remains centered in the tube. This may be an iterative step.

7. From the position of the instrument as shown in figure $4 B$, rotate the instrument $180^{\circ}$ and check to see if the bubble is still centered in the tube. If so, the plate bubble tube does not have to be adjusted (fig. $4 C$ )

8. If the bubble is substantially off center (not level), adjust the bubble with the bubble tube-leveling screws so that the bubble comes halfway back to the center.

9. Use nut $\mathrm{c}$ to return the bubble to the center.

10. Repeatedly check the bubble's position following steps 3 through 5; continue to step 6 when the bubble remains centered in the tube. Because the plate bubbleadjusting process may be iterative, you may need to also complete steps 7 through 9 .

11. Once the cylindrical plate bubble tube of the total station is adjusted, the instrument is now level; that is, the mechanical vertical axis of the total station is aligned with the vertical direction, which is the direction of gravity in surveying.

12. Check the position of the circular bubble level in the tribrach (if it has one). It should be centered within the bullseye of the circular level. If not, adjust the circular bubble adjusting screws until the level bubble of the tribrach is within the bullseye.

At this point, the plate-level tube of the total station and the circular bubble of the tribrach should be precisely adjusted. A quick check to determine if the level bubbles were properly adjusted is to re-level the instrument and verify that both bubbles are in the level position. This procedure can be 
$\boldsymbol{A}$

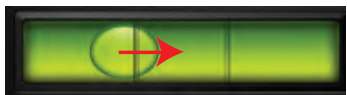

$\oplus$

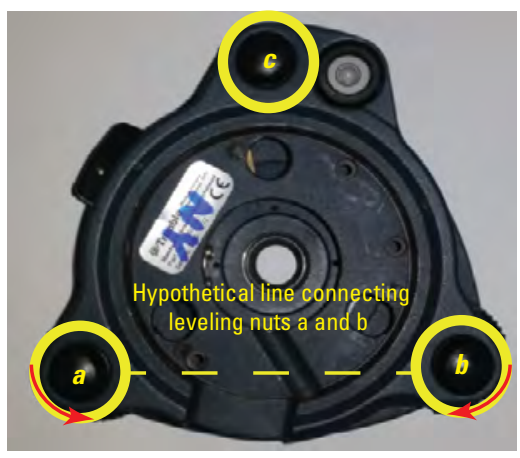

Position of operator

C

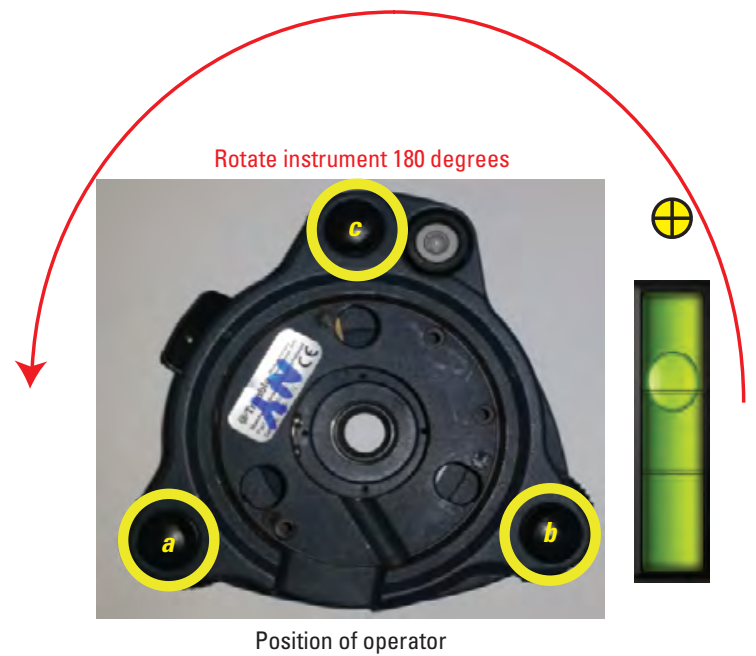

D

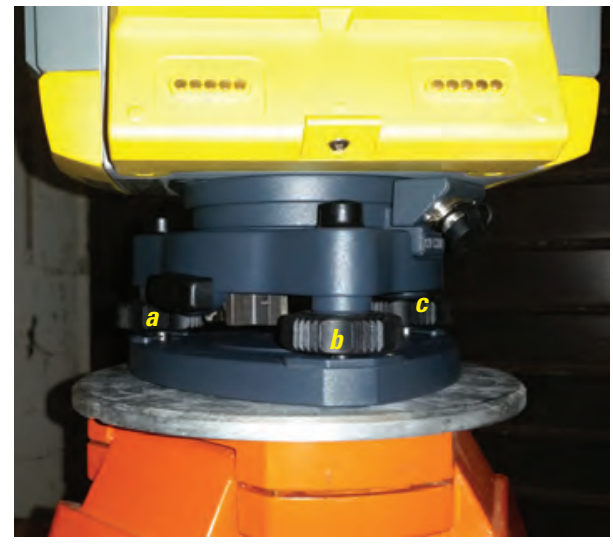

Profile view of total station, tribrach with leveling nut identifier, and tripod platform.
B

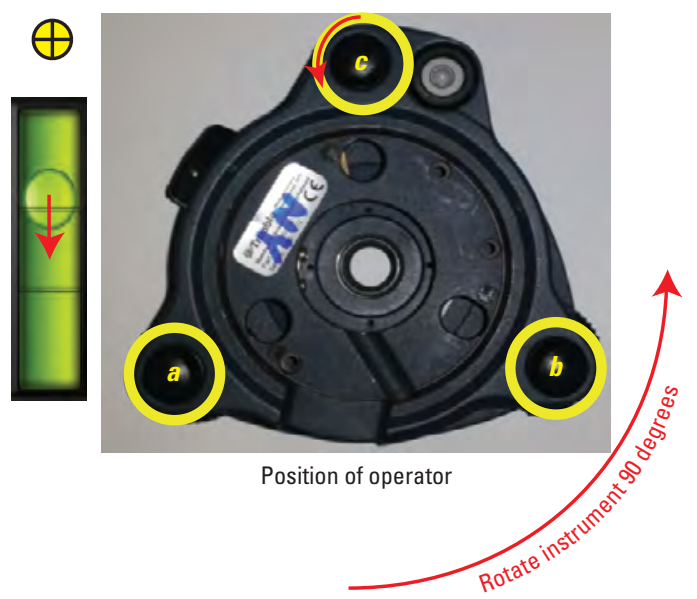

Bubble tube level after 180-degree rotation

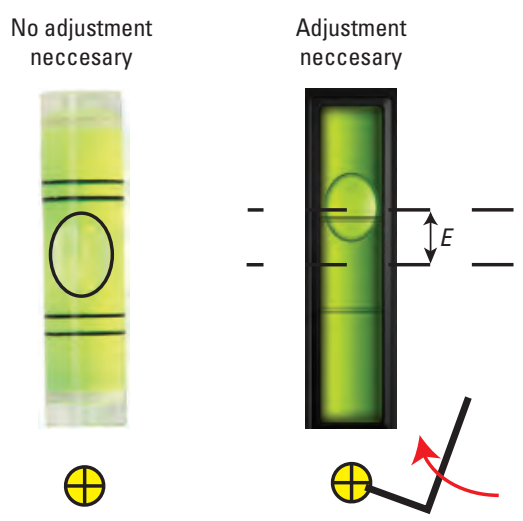

Adjust bubble-tube leveling screws so the bubble comes halfway back to center, or $0.5 \times E$

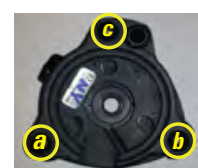

\section{EXPLANATION}

Tribrach and leveling nut identifier

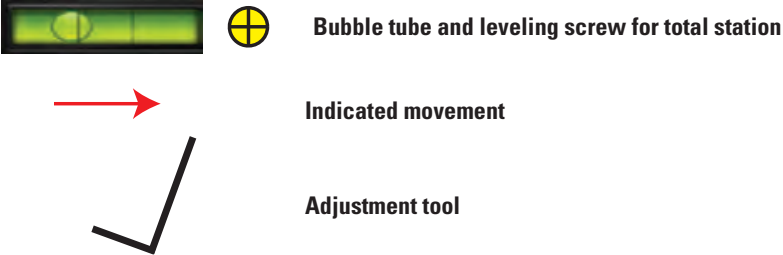

Figure 4. Method to calibrate a bubble tube level of a total station. This procedure can be modified for total stations with a circular (bullseye) level and for total stations with an electronic level. $A$, tribrach and plate bubble tube; $B$, tribach and plate bubble tube after rotating 90 degrees; $C$, tribrach and plate bubble tube after rotating 180 degrees; and $D$, profile view of a total station, tribrach with leveling nuts identified, and tripod platform. The total station is not shown in $A, B$, and $C$ for illustrative purposes. 
modified for tribrachs that have a plate-level tube instead of a bullseye level. The level-bubble adjustment method is efficient because the cylindrical plate-bubble tube of the total station and the circular bubble of the tribrach can both be adjusted during the same procedure. Additionally, because proper adjustment of the cylindrical plate-bubble tube is a prerequisite for taking measurements with the total station (fig. 4, steps 1-10), the circular level of the tribrach can be checked and adjusted (fig. 4; step 12) as part of the normal workflow of a trigonometric-leveling survey with minimal additional work.

Reference the total station's operation manual for procedures to calibrate electronic level bubbles because the method may vary among instrument models or manufacturer. The basic procedure for leveling with an electronic bubble (or tilt sensor) is the same as with the analog cylindrical plate bubbles. There is a variation in this procedure depending on whether the instrument displays tilt only with or without a digital representation of the cylindrical bubble tube or whether there is only a representation of a circular bubble. Sometimes the user can select between variations of the bubble. If possible, select the tilt values, a cylindrical bubble tube, or both.

Once the tilt values, representation of the cylindrical bubble tube, or both have been selected, begin the leveling procedure by aligning the panel of the total station having the display and keyboard parallel to the previously described hypothetical line connecting leveling nuts $\mathrm{a}$ and $\mathrm{b}$ of the tribrach (fig. 4A). Turn the leveling nuts a and b clockwise or counterclockwise (but always in the opposite direction) until the digital representation of the cylindrical bubble is centered (level), the indicated tilt is $00^{\prime \prime}$, or both. Next, rotate the instrument $90^{\circ}$, and center (level) the bubble using leveling nut c (fig. 4). Then, return to the original position and refine the centering (level) or tilt display to $00^{\prime \prime}$, again return to the $90^{\circ}$ orientation, and refine the centering (level). When you are satisfied that the bubble is centered (level) or is displaying $00^{\prime \prime}$ in these two positions, turn to $180^{\circ}$ from the original position. Any movement of the bubble is twice the actual error in the tilt sensor. In general, if a tilt of $40^{\prime \prime}$ is indicated by the electronic bubble, the actual error is half the indicated tilt, or $20^{\prime \prime}$ for this example (Paiva, 2016). Remove half of the tilt, or bubble movement from center, with leveling nuts a and $b$. Then turn $90^{\circ}$ to the position where the instrument display was perpendicular to the original $90^{\circ}$ position. Remove half of the tilt or bubble movement from center with leveling nut c. Repeat this iterative process until the electronic cylindrical bubble is centered (level) in both orientations or until the tilt display is reading $00^{\prime \prime}$. If you know how to perform a zero set (on some motorized instruments this is an automatic function), follow that procedure to remove the error in the tilt sensor.

If the electronic bubble or tilt sensor value is displayed as a circular vial only, align the display so that it is parallel to the hypothetical line connecting leveling nuts a and b (fig. 4). Center (level) the bubble and rotate to $180^{\circ}$. Any bubble movement is twice the error in the tilt sensor. Move the bubble back halfway to the center with the leveling nuts. The instrument is now level but can be confirmed by returning it to the original position. The bubble should be in the same place. If you know how to remove the error in the tilt sensor (often called the "tilt sensor zero set"), use the manufacturer's instructions as provided in the user's manual. Electronic bubbles should be precisely adjusted before every trigonometric-leveling survey and multiple adjustments per survey may be required when the daily air temperature range exceeds 20 degrees Fahrenheit $\left({ }^{\circ} \mathrm{F}\right)$ (Paiva, 2016). Circular bubble levels that are mounted to adjustable-height tripods can be adjusted using the method adopted by Rydlund and Densmore (2012) and from Henning (2010; fig. 5).

\section{Plummets}

Optical plummets are used to precisely center the surveying instrument over a survey marker. There are two types of optical plummets for surveying instruments: plummets that rotate with the instrument when it is pointed at a target and plummets that remain stationary when the instrument is rotated. Typically, plummets that rotate with the instrument are built into the alidade (rotating part of the instrument), and stationary plummets are built into the tribrach. In either case, optical plummets are susceptible to shock and vibration and must be routinely inspected and adjusted to ensure that the optical axis of the plummet is aligned with the plumb line or vertical axis of the instrument (U.S. Department of the Army, 2001).

For stationary optical plummets, or plummets that remain in a fixed position when the instrument is rotated, a plumb bob can be suspended from the hook attached to the bottom of the central fixing screw of an adjustable tripod. Some effort may be required to ensure the tripod platform is level before attaching the plumb bob to the hook. Substantial tilts of this surface will cause your check and adjustment of the optical plummet to be erroneous. A precisely adjusted torpedo level, which is just a smaller version of a spirit level, can be used to verify the tripod platform is level; note that this is not the same as carefully leveling the instrument on the tripod. Once the adjustable tripod and the instrument that has the stationary plummet are level, carefully mark a point on the ground directly beneath the plumb bob, and then remove the plumb bob from the tripod (Brinker and Minnick, 1995). The crosshairs of the optical plummet should coincide with the set plumb mark; if not, an adjustment is necessary. Use the plummet adjusting screws, typically four screws on the eyepiece, to align the plummet crosshairs with the plumb mark. Reset and relevel the instrument; repeat the procedure a few times until the crosshair of the optical plummet consistently aligns with the plumb mark.

For optical and laser plummets in the alidade, the plummet adjustment is easily checked, and the instrument does not have to be level. However, the tripod must be firmly anchored into the ground. Mark the point on the ground where the plummet crosshairs (or laser spot) strikes (fig. $6 A$ ). If this is an optical plummet adjustment, it is helpful to have an assistant mark the location. Once the location has been marked, rotate the instrument $180^{\circ}$ (fig. $6 B$ ). Any apparent movement is twice 


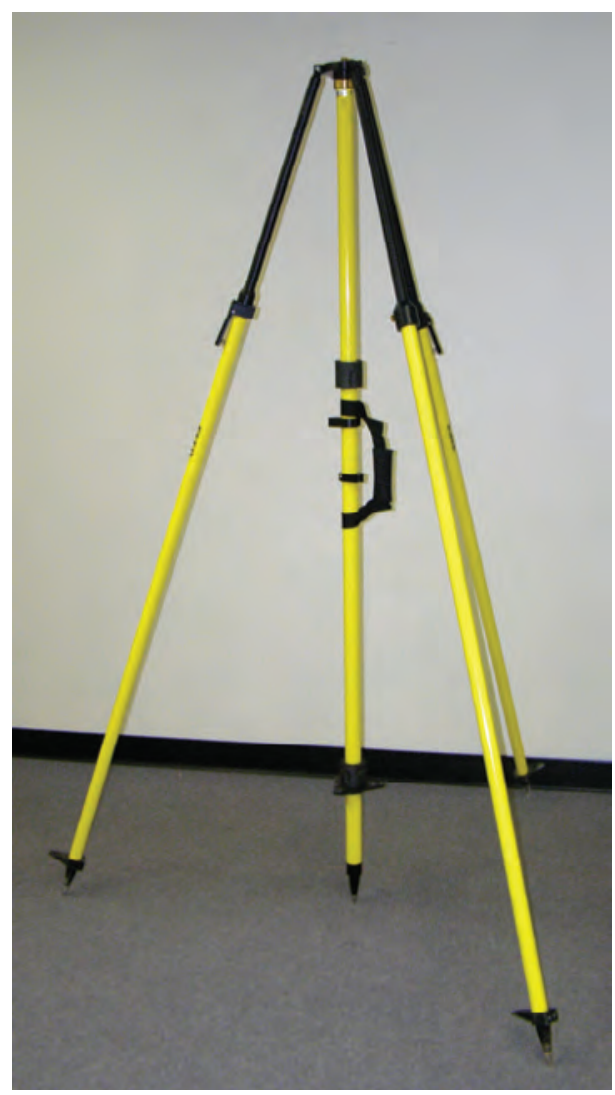

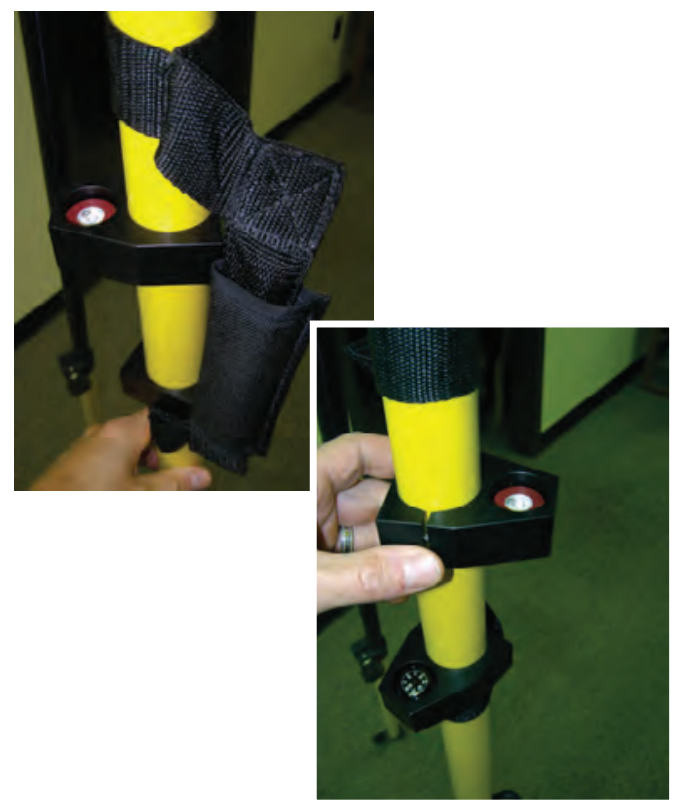

\section{EXPLANATION}

Check and adjustment of circular level vial on fixed-height tripod

1. Turn center pole 180 degrees after centering bubble on center pole.

2. If any part of the bubble goes out of the black circle, move quick release legs until bubble is halfway between position 1 and position 2 .

3. Use adjusting screws until bubble is centered.

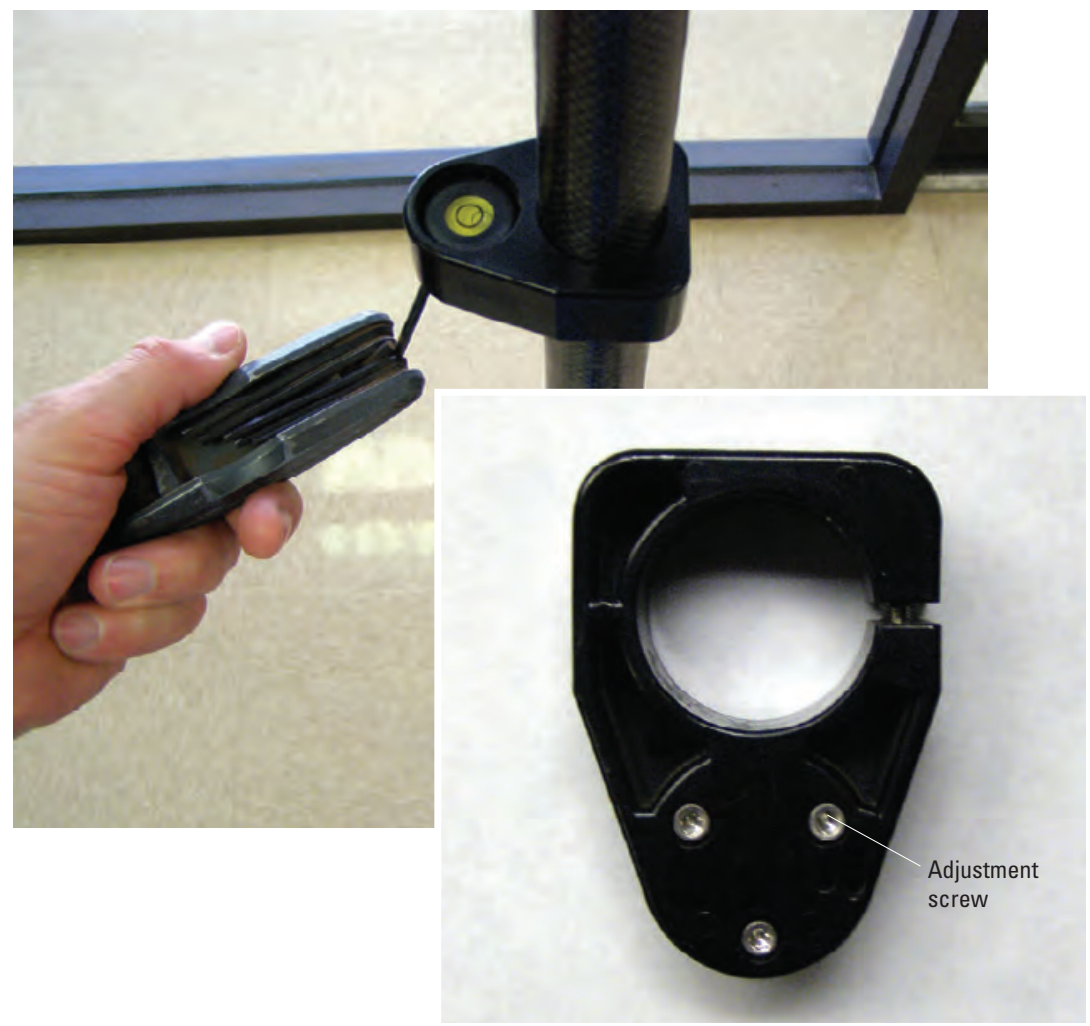

○ Adjustment with legs

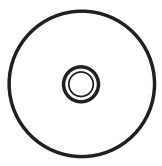

Position 1 and 2 after adjusting screws

Figure 5. Circular level vial adjustment on fixed-height center poles. Modified from Rydlund and Densmore (2012) and Henning (2010). 
$\boldsymbol{A}$

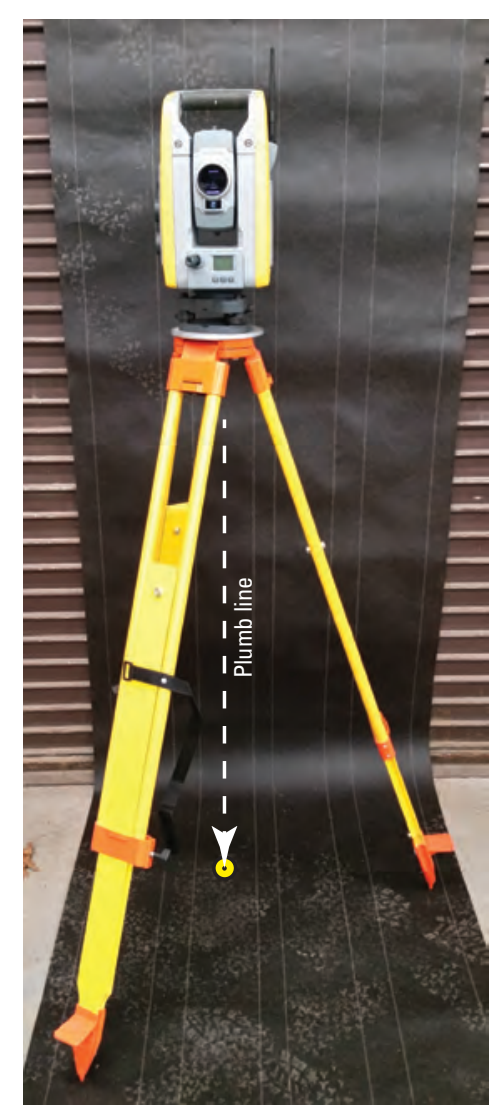

Mark the point on the ground where the plummet crosshairs (or laser spot) strikes
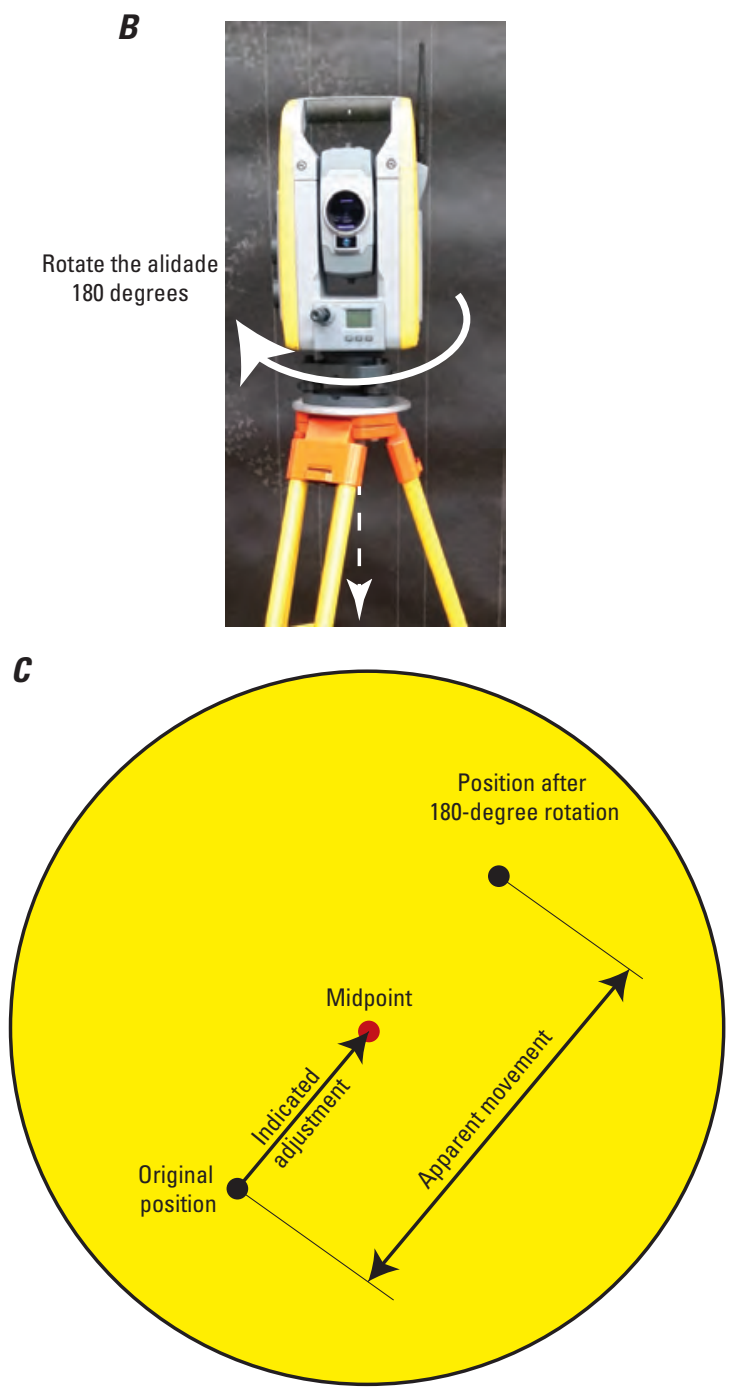

D

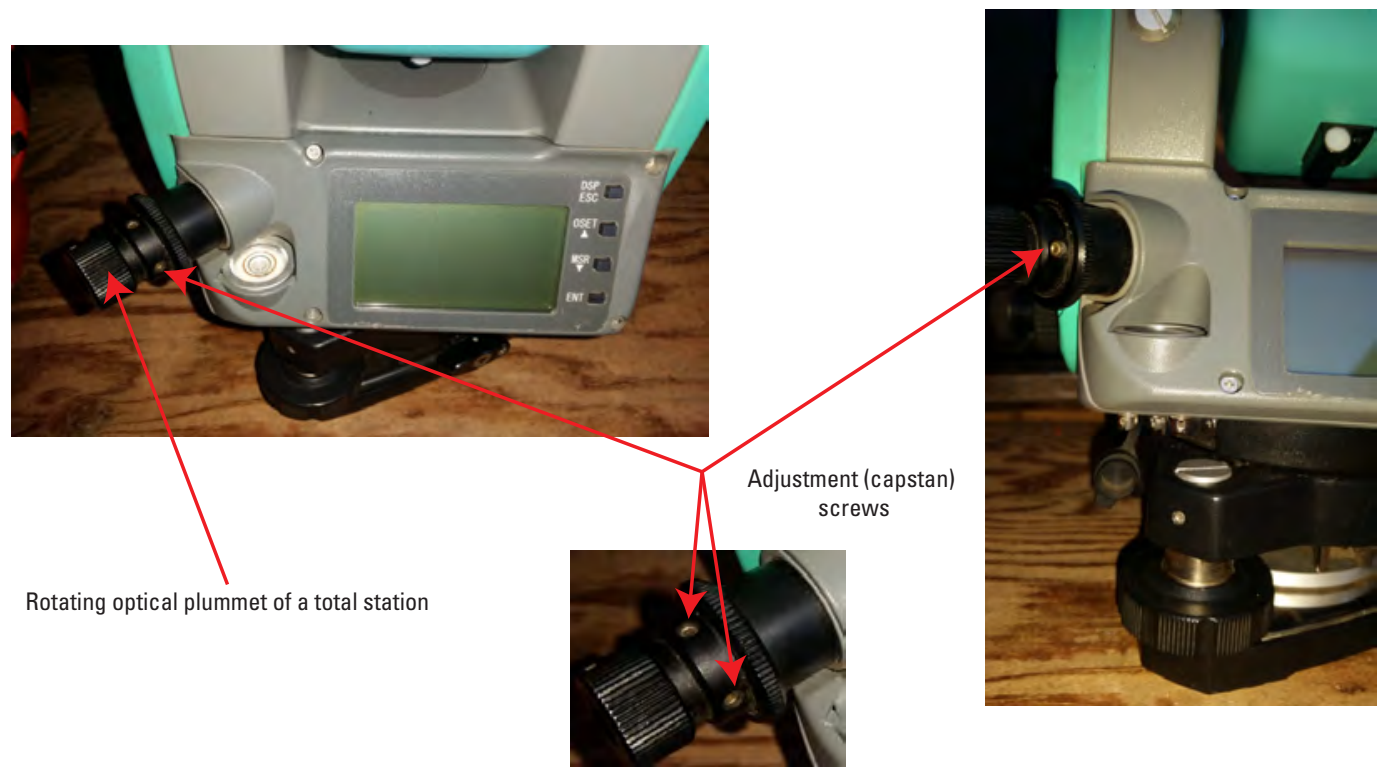

Figure 6. Method to adjust rotating optical or laser plummet (Paiva, 2016). $A$, plumb line and ground point where plummet crosshairs strike; $B$, rotating the total station 180 degrees; $C$, apparent movement after 180 -degree rotation; and $D$, rotating the optical plummet of a total station and the adjustment screws. 
the actual error of the plummet (fig. 6C). Generally, an apparent movement of $1 \mathrm{~mm}(0.039 \mathrm{inch}$ [in.]) or less is considered within the limits of adjustment. If the apparent movement is more than $1 \mathrm{~mm}$, adjust the crosshairs (or laser spot) to the midpoint between the apparent movement location and the original location. If the point on the ground is marked with a wooden stake, the original position and the apparent movement position can be marked, and the midpoint of the line between these two points can also be marked. Then, use the adjusting screws on the optical plummet eyepiece to move the crosshairs to the halfway point (fig. $6 C, D$ ). As with all adjustment procedures, repeat this process at least once more. For less experienced operators, this may have to be an iterative process of several cycles.

Tripods with adjustable center poles eliminate the need for the optical and laser plummets because the center pole forces proper alignment of the vertical axis of the survey instrument over the survey marker. However, if the level bubble on the tripod, tribrach, or center pole is not properly adjusted, the instrument may appear to be centered over the survey marker, but in fact, may be off center. See figure 5 for an explanation of the checking and adjusting procedure. It is mandatory that the level bubbles (both plate bubble, electronic bubble or tilt sensor or electronic circular bubble) be adjusted before checking or adjusting the center-pole bubble because centering by this method can be affected by an instrument that is not perfectly level. Instrument components such as a level bubble and plummets need to be routinely cleaned and inspected for damage, especially after use or transport, and before a trigonometric-leveling campaign.

\section{Testing Total Station Components Before a Trigonometric-Leveling Campaign}

Manufacturers of surveying instruments recognize that even the most precise total stations have construction defects or other imperfections that can affect the quality of survey measurements. The implication of these construction defects are identified by the manufacturer on specification tables that indicate constant and distance-weighted errors caused by imperfections of the EDMI measuring system, angular uncertainty that is associated with the misalignment of the instrument axes, and other technical characteristics of the total station. It is crucial for the surveyor to understand these error sources and the procedures that can determine the resulting uncertainty. In many cases, the uncertainty can be reduced or eliminated before a surveying campaign, or at a minimum, it can be determined so that the surveyor operates within identified parameters for quality measurements. For example, the operator may elect to reduce measurement distances to minimize the effect of distance-weighted error inherent in the EDMI measuring system. Reducing measurement distances may also lessen the effect of angular error resulting from axial defects, such as vertical and horizontal collimation error described in subsequent sections, because these angular errors are proportional to the measurement distance.

Modern total stations have the capacity to determine, store, and automatically apply corrections for axial defects using integrated software programs and routines that are identified by the manufacturer (Leica Geosystems, 2008). Authoritative literature recommends calibrating a total station when axial defects result in a difference between F1 and F2 horizontal and vertical circle readings that exceeds 30" (Federal Geodetic Control Committee [FGCC], 1984; USACE, 2007). Because trigonometric-leveling surveys require total stations that can measure precision zenith angles, angular uncertainty resulting from misalignment of instrument axes must be determined before each survey regardless of the difference between $\mathrm{F} 1$ and $\mathrm{F} 2$ circle readings.

\section{Testing Axial Alignment and Scales}

The accuracy of the horizontal and zenith angle measurements of a total station can be affected by systematic defects in the axial alignment of one or more of the three instrument axes (Ogundare, 2016). The three major axes of the instrument, which must be precisely oriented for quality angular measurements, are (1) the optical, or line-of-sight axis, that must be perpendicular to the horizontal (tilting or trunnion) axis, (2) the tilting (horizontal or trunnion) axis that must be perpendicular to the vertical axis, and (3) the vertical (standing) axis that must be aligned with the direction of apparent gravitational force at the instrument location or be orthogonal to the horizontal plane of a perfectly level instrument (fig. 7). Furthermore, the horizontal and vertical circles (scales) of the total station must also precisely align with their respective axis to ensure the observed angle represents the true direction to the target.

Testing procedures such as taking horizontal and zenith angle measurements in F1 and F2 can help determine if one or more of the instrument axes are defective. Once the defect is determined, the adjustment can be stored in the total station and will be automatically applied to subsequent angle measurements. In some cases, the angular error caused by axial defects can be canceled out by averaging the F1 and F2 horizontal and zenith angle measurements, also called horizontal and vertical scale observations, respectively. An axial defect can be caused by the inevitable human and mechanical imperfections that occur during instrument construction, by damage during use and transport, or by both. Sources of uncertainty caused by axial defects are identified as vertical collimation error, horizontal collimation error, and tilting-axis error (USACE, 2007; Ogundare, 2016). For all these sources of uncertainty, the determined error will have the opposite sign of the correction; for example, if a vertical collimation error $+00^{\circ} 00^{\prime} 40^{\prime \prime}$ is determined, a correction of $-00^{\circ} 00^{\prime} 40^{\prime \prime}$ will be applied to the adjusted zenith angle. Sources of uncertainty caused by defective axial alignment, and procedures to determine and account for these errors, are described in the 


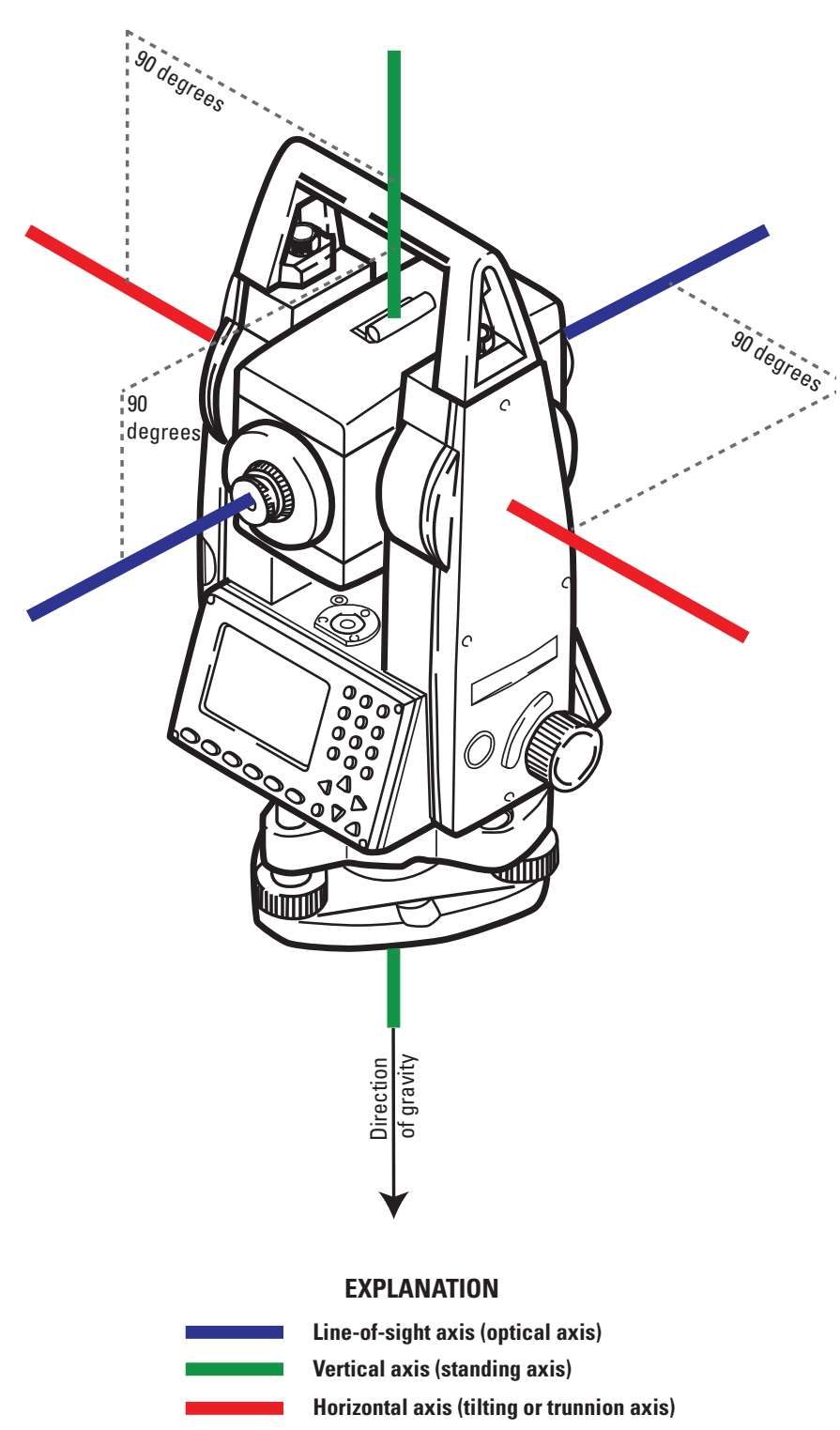

Figure 7. Orientation of three instrument axes of a total station. Modified from Leica Geosystems, part of Hexagon, (2013); used with permission.

following section. These procedures can be used for most total stations, but the operator should also consult the user manual for methods that are specific to their instrument model.

\section{Vertical Collimation Error}

Vertical collimation error is a defect that causes a misalignment between the true and observed (apparent) line of sight along the vertical plane of the instrument, which can be measured on the vertical circle, and will result in an erroneous zenith angle (fig. 7; Ogundare, 2016). For a perfectly collimated instrument, a vertical circle reading (zenith angle) of zero should precisely align with the upward vertical axis of the total station and the crosshair of the telescope (Ogundare, 2016). A deviation from this condition may be caused by vertical collimation error. This error has also been identified as vertical circle (or scale) error and vertical index error in some literature sources. The VD, which is the height difference between the horizontal plane (axis) of the total station and the horizontal plane of the center of the target (prism), is calculated by multiplying the slope distance by the cosine of the zenith angle measured from the total station to the target (fig. 8); therefore, it is critical to precisely determine and account for vertical collimation error before initiating a trigonometric-leveling survey to ensure quality zenith angles. In most cases, taking zenith angle measurements in F1 and F2 can eliminate vertical collimation error because the error in the F1 observation cancels out the error in the F2 observation because they are equal, although in opposite directions. Once determined, the vertical collimation error can also be stored in the integrated or external data-collection device (but never both) so that the adjustment is automatically applied to subsequent vertical circle readings (zenith angles) (Leica Geosystems, 2013). The procedure to determine vertical collimation error (figs. $9 A$ and 10) is as follows:

1. Set up the total station. Ensure the instrument is level.

2. Set up a calibrated rod (or other type of target) about $164 \mathrm{ft}(50 \mathrm{~m})$ from the total station and ensure that it is level. The staff will have to be elevated above the horizontal plane of the total station on an elevated platform or hillside.

3. In F1, vertically rotate the instrument about $10^{\circ}$ above the horizontal plane. Take a reading on the rod and record the zenith angle $(z 1)$. An example of the zenith angle reading is $80^{\circ} 00^{\prime} 20^{\prime \prime}$.

4. In F2, point the instrument at the rod reading from step 3, which is $13.20 \mathrm{ft}(4.0 \mathrm{~m})$ for this example (fig. 10). Record the zenith angle (z2). An example of the zenith angle reading is $280^{\circ} 00^{\prime} 20^{\prime \prime}$. Four to six sets of F1 and F2 observations should be made, and then averaged to determine $z 1$ and $z 2$. Obvious outliers should be discarded.

5. Calculate the vertical collimation error $(v)$ using the following equation and the example zenith angles from steps 3 and 4:

$$
v=\left(z 1+z 2-360^{\circ}\right) \div 2
$$

$$
v=\left(80^{\circ} 00^{\prime} 20^{\prime \prime}+280^{\circ} 00^{\prime} 20^{\prime \prime}-360^{\circ}\right) \div 2
$$

$$
\nu=+20^{\prime \prime}
$$

where

$v \quad$ is the defect, or vertical collimation error, in arc-seconds;

$z 1$ is the F1 zenith angle, in degrees, minutes, and seconds; and

$z 2$ is the F2 zenith angle, in degrees, minutes, and seconds. 


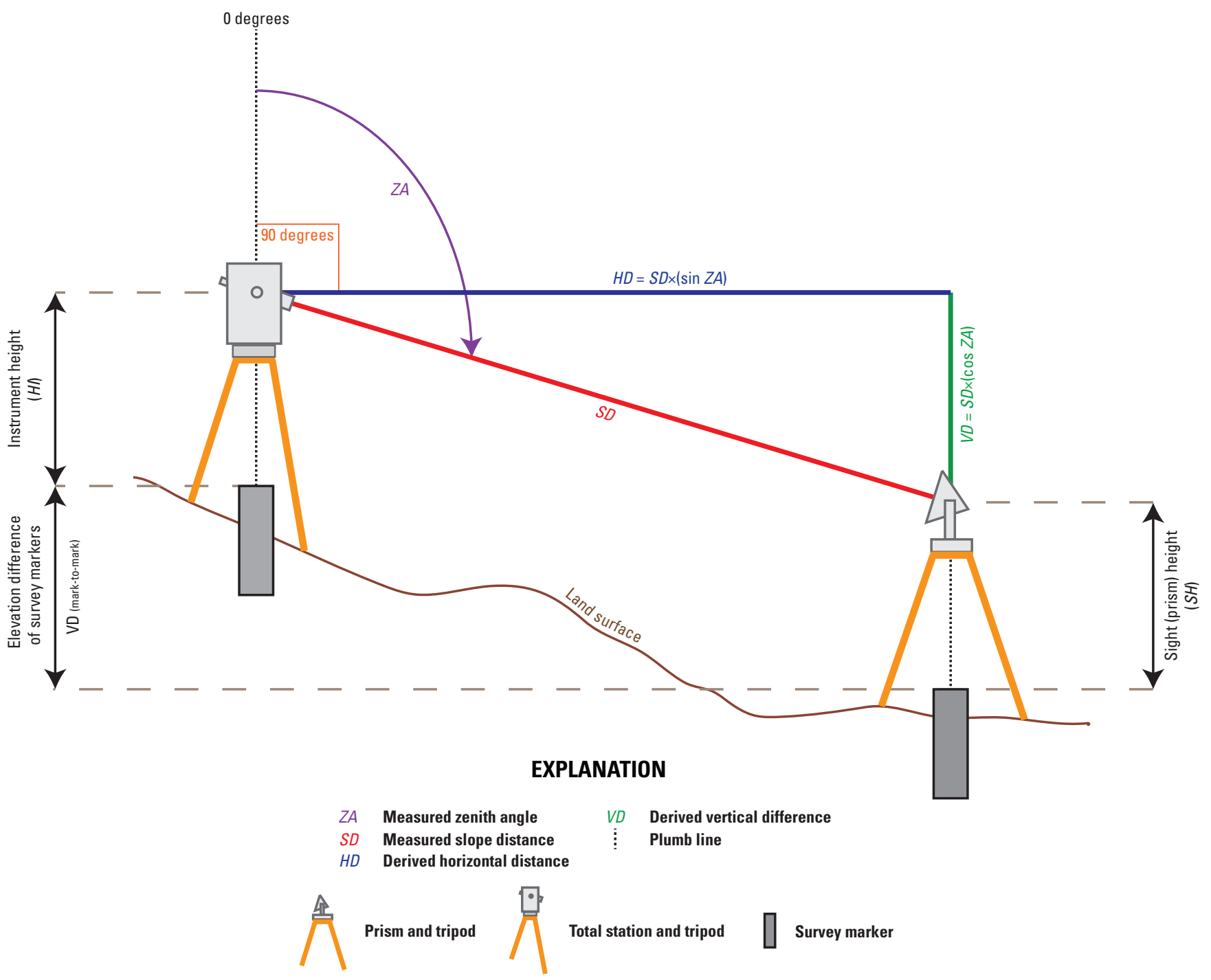

Figure 8. Geometry of a total station measurement. Modified from U.S. Army Corps of Engineers (2007); used with permission.

6. Because the vertical collimation error has the opposite sign of the correction, subtract $20^{\prime \prime}$ from the F1 reading to get the adjusted zenith angle $(z 1[$ adj $])$; for example, $z 1($ adj $)=80^{\circ} 00^{\prime} 20^{\prime \prime}-00^{\circ} 00^{\prime} 20^{\prime \prime}=80^{\circ} 00^{\prime} 00^{\prime \prime}$.

7. Follow the instructions in the instrument manual to store the vertical collimation error $(v)$, or defect, in the total station so that the correction is automatically applied to subsequent zenith angle measurements.

8. If the instrument does not have the ability to store this error and automatically apply corrections to the vertical circle readings, the most efficient way to apply the correction is after completing the field work, unless there is a need to accurately determine an elevation in the field.

Vertical collimation error is the most important defect for a surveyor to determine because an erroneous zenith will increase the uncertainty of the VD, which will subsequently affect the quality of the elevation of the survey marker (fig. 8).
In the following sections, horizontal collimation and tiltingaxis error are discussed. These defects do not directly affect the uncertainty of the VD like vertical collimation error but could have unintended consequences that adversely affect the quality of observations. For example, Noll and Chu (2017) determined in areas with steep topography, a horizontal position error of less than $0.1 \mathrm{ft}$ affected the accuracy of a measured elevation. Topography of an earthen embankment (used to retain water in the basin of a reservoir) with a downslope angle of $62^{\circ}$ was surveyed. The objective of the study was to detect temporal change in the topography of an earthen embankment by precisely measuring land-surface elevations with a total station. Two topographic surveys were completed during a 44-month period, which required resurveying previously measured locations on the earthen embankment using a stake-out method, which is the industry standard for accurately determining locations that were previously surveyed. Previously surveyed locations were re-measured within 
$\boldsymbol{A}$

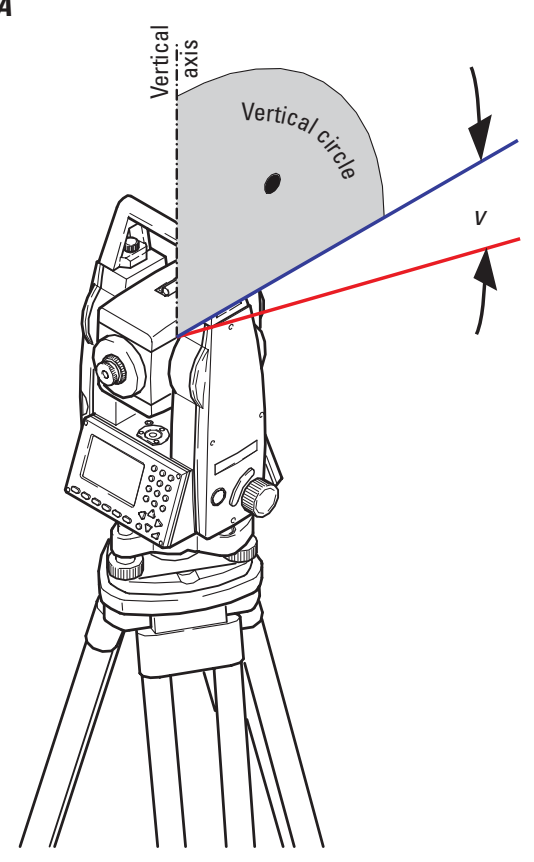

B

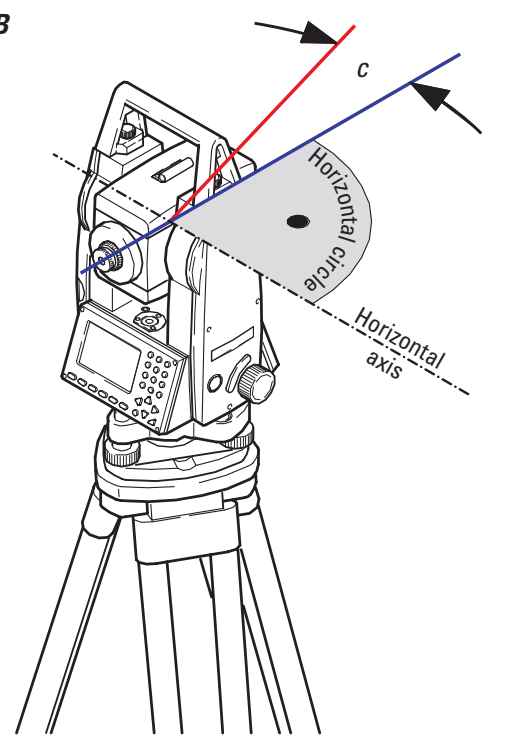

$c$

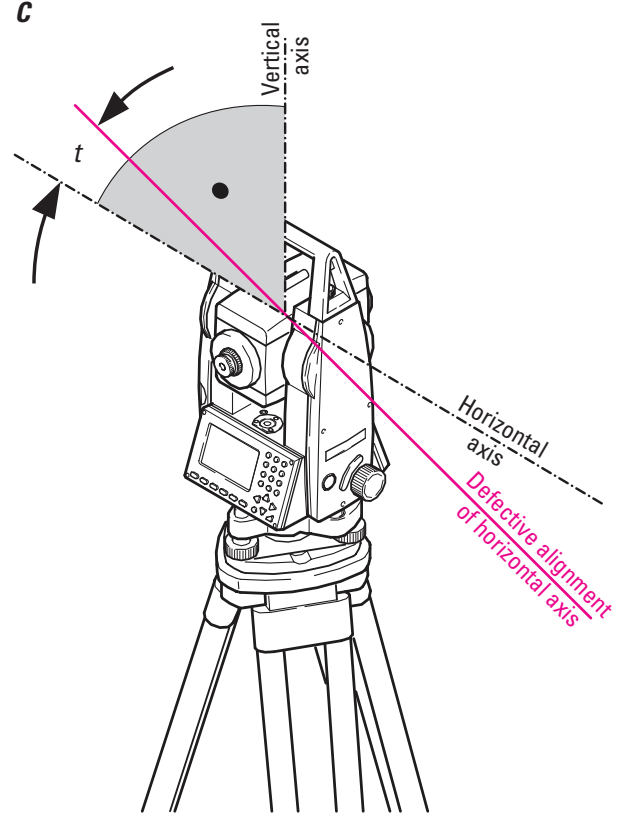

D

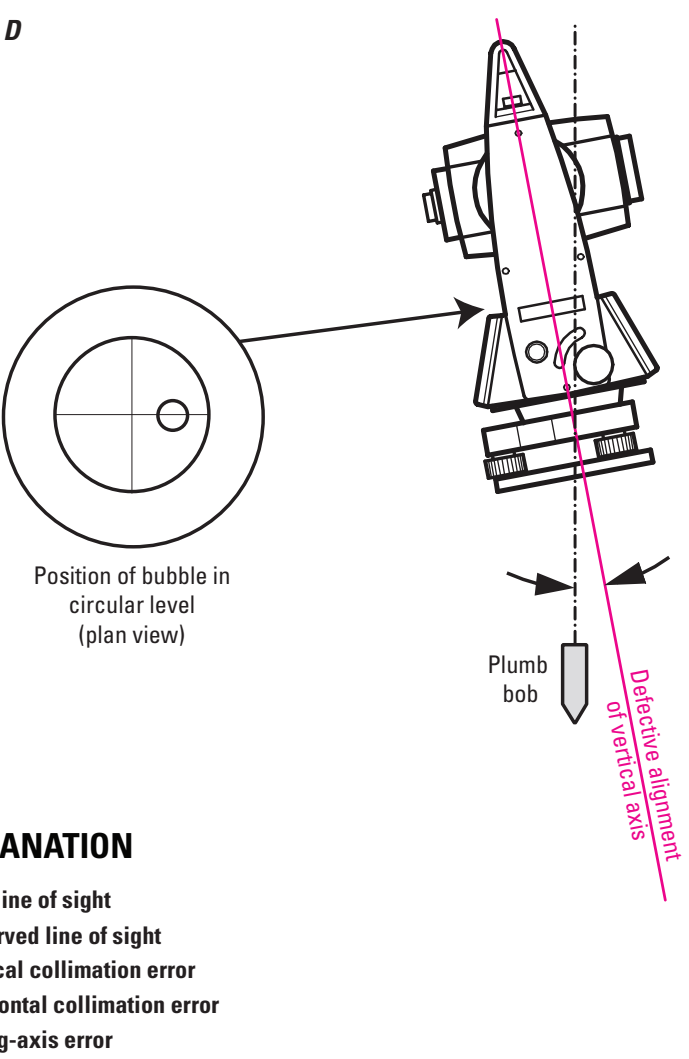

\section{EXPLANATION}

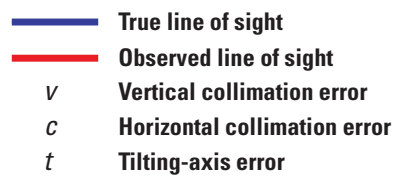

Figure 9. Common systematic sources of error for total station instruments. $A$, vertical collimation error; $B$, horizontal collimation error; $C$, tilting-axis error; and D, vertical-axis error. Modified from Leica Geosystems, part of Hexagon, (2013) and Ogundare (2016); used with permission. 


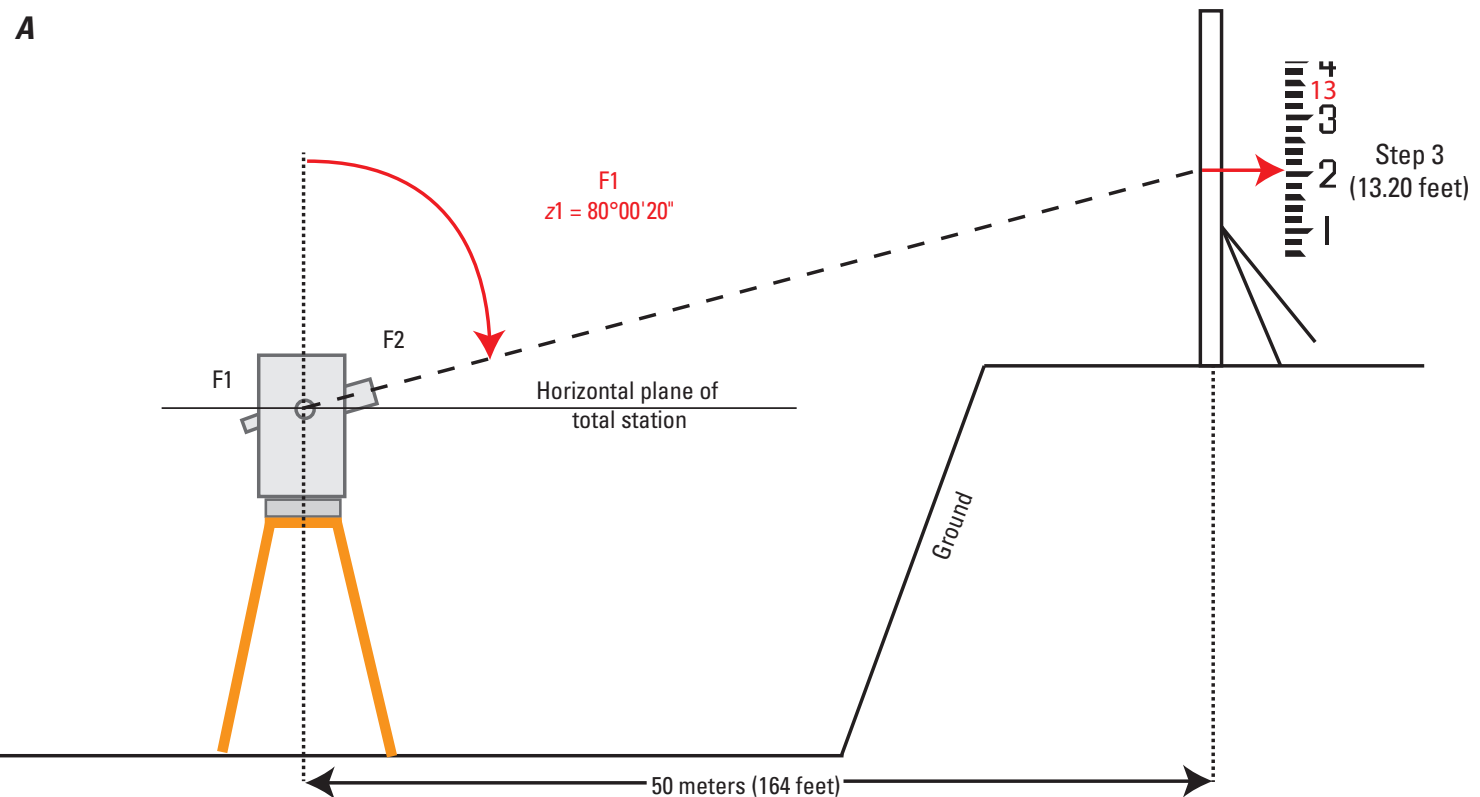

1. Set up the total station. Ensure the instrument is level.

2. Set up a calibrated rod or staff about 164 feet from the total station and make sure that is level.

3. In direct instrument orientation (F1), vertically rotate the instrument approximately 10 degrees above the horizontal plane.

Take a reading on the rod and record the zenith angle (z1). An example of the zenith angle reading is $80^{\circ} 00^{\prime} 20^{\prime \prime}$.

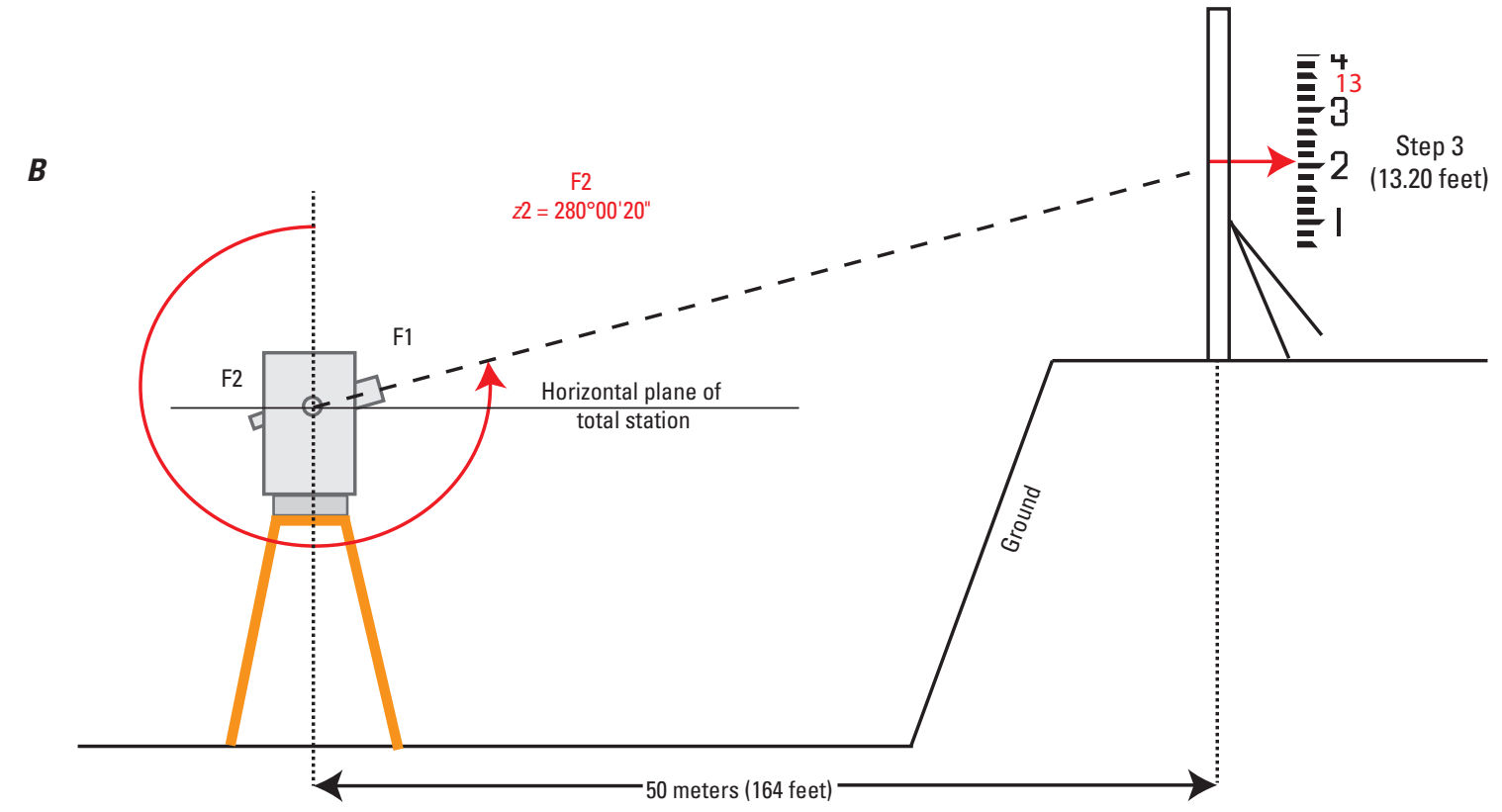

4. In reverse instrument orientation (F2), point the instrument at the rod reading from step 3 , which is 13.20 feet for this example.

Record the zenith angle (z2). An example of the zenith angle reading is $280^{\circ} 00^{\prime} 20^{\prime \prime}$.

5. Calculate vertical collimation error $(v)$ using the following formula: $v=\underline{z 1+z 2-360^{\circ}}$

\section{EXPLANATION}

_ - - Line of sight from total station to prism

Plumb line

Total station and tripod

Calibrated staff

Figure 10. Method for determining vertical collimation error $(v)$ of a total station. $A$, zenith angle measurement from the total station to the calibrated staff in direct instrument orientation $(F 1)$; and $B$, zenith angle measurement from the total station to the staff in reverse instrument orientation (F2). Four to six sets of F1 and F2 observations should be made and then averaged to determine $z 1$ and $z 2$. 
$0.1 \mathrm{ft}$ (horizontal) of the original surveyed location; however, because of the steep downslope angle $\left(62^{\circ}\right)$, the horizontal uncertainty $(0.1 \mathrm{ft})$ created additional vertical uncertainty of more than $0.05 \mathrm{ft}$. For this reason, axial defects that affect horizontal circle readings are described in the following sections.

\section{Horizontal Collimation Error}

Horizontal collimation error, also referred to as lineof-sight error, is an axial defect that causes a misalignment between the true and observed (apparent) line of sight along the horizontal plane of the instrument, which can be measured on the horizontal circle, and will result in an erroneous horizontal angle (figs. 7 and 9B; Ogundare, 2016). This axial defect causes the line-of-sight axis to be out of perpendicular alignment with the tilting (horizontal or trunnion) axis so that horizontal circle readings are not representative of the true direction to the target. Because the line-of-sight axis should be orthogonal to the tilting axis, the deviation angle from $90^{\circ}$ is considered the horizontal collimation error (figs. 9 and 11). If an error is detected, an angular adjustment must be applied to the horizontal circle reading to ensure the adjusted angle represents the true direction to the target location. Horizontal collimation error is often difficult to determine in the field because the crosshair of the telescope seems to be properly aligned with the target but is actually deviated from the true direction to the target. The horizontal collimation error can be determined using the following procedure (fig. 11):

1. Adjust the vertical circle reading of the total station for vertical collimation error using the previously described procedure (in the "Vertical Collimation Error" section).

2. Set up the total station. Ensure the instrument is level.

3. Set up a calibrated rod (or other type of target) horizontal to the ground about $164 \mathrm{ft}(50 \mathrm{~m})$ from the total station and ensure that it is level and aligned with the horizontal plane of the total station.

4. In F1, point the instrument at the rod and take a reading where the vertical crosshair of the telescope aligns with a rod increment. Ensure the zenith angle is reading $90^{\circ}$ when the telescope is pointed at the rod. Record the horizontal angle $(H z 1)$. An example of the horizontal angle reading is $42^{\circ} 45^{\prime} 48^{\prime \prime}$. The rod reading for this example is $3.19 \mathrm{ft}$.

5. In F2, point the instrument at the rod increment from step 4 (3.19 $\mathrm{ft}$ for this example) and record the horizontal angle $(H z 2)$. Ensure the zenith angle is reading $270^{\circ}$. An example of the horizontal angle reading is $222^{\circ} 45^{\prime} 36^{\prime \prime}$. Four to six sets of F1 and F2 observations should be made and then averaged to determine $H z 1$ and $H z 2$. Obvious outliers should be thrown out. Use the following equation to determine the horizontal collimation error when the zenith angle is $90^{\circ}$ :

$$
c=\left(H z 1-\left[H z 2-180^{\circ}\right]\right) \div 2
$$

where

$$
\begin{gathered}
\text { c is the defect, or horizontal collimation error, } \\
\text { in arc-seconds; } \\
H z 1 \quad \text { is the F1 horizontal angle measurement, in } \\
\text { degrees, minutes, and seconds; and } \\
\mathrm{Hz2} \text { is the F2 horizontal angle measurement, in } \\
\text { degrees, minutes, and seconds. }
\end{gathered}
$$

6. Follow the instructions in the instrument manual to enter the horizontal collimation error (c) so that a horizontal angle adjustment is automatically applied to the circle reading to account for axial defect.

7. Note that if $c$ is positive, enter the horizontal collimation error as a negative value, so that the adjusted F1 horizontal angle is less than the original observed angle.

8. If the instrument does not have the ability to store this error and automatically apply corrections to the horizontal circle readings, the most efficient way to apply the correction is after completing the field work, unless there is a need to accurately determine an elevation in the field.

As described in the procedure (fig. 11), the horizontal collimation error $(\mathrm{c})$ can be determined if the zenith angle is $90^{\circ}$ of a perfectly level instrument that has been adjusted for vertical collimation error. The horizontal collimation error is compounded by the zenith angle $(z)$ when the telescope is tilted from the horizontal plane (zenith angle $\neq 90^{\circ}$ ); the horizontal collimation error can be determined for any zenith angle $\left(C_{z}\right)$ using the following equation:

$$
C_{z}=c \div \sin (z)
$$

where

$c$ is the defect, or horizontal collimation error, in arc-seconds;

$C_{z} \quad$ is the horizontal collimation error for a zenith angle, in arc-seconds; and

$z \quad$ is the zenith angle, in degrees.

As the equation indicates, if a small horizontal collimation error exists when the zenith angle of the instrument is reading $90^{\circ}$, this error will become progressively larger for steeper sightings to a target. (The horizontal collimation error actually approaches infinity as the zenith angle approaches zero [or nadir at $180^{\circ}$ ] and the telescope [line-of-sight axis] aligns with the vertical plane of the instrument; however, these sighting angles are not encountered in the field.) Consider an example scenario in which a total station is used in a location with highly variable topography where the telescope needs to be substantially tilted with respect to the horizontal plane. In this example, a horizontal collimation error (c) of $30^{\prime \prime}$ (when the line of sight is horizontal, or a zenith angle of $90^{\circ}$ in F1) would become $42^{\prime \prime}\left(C_{z}\right)$ when sighting at a target when the zenith angle is $45^{\circ}$. Given a measurement distance of $500 \mathrm{ft}$, 
$\boldsymbol{A}$

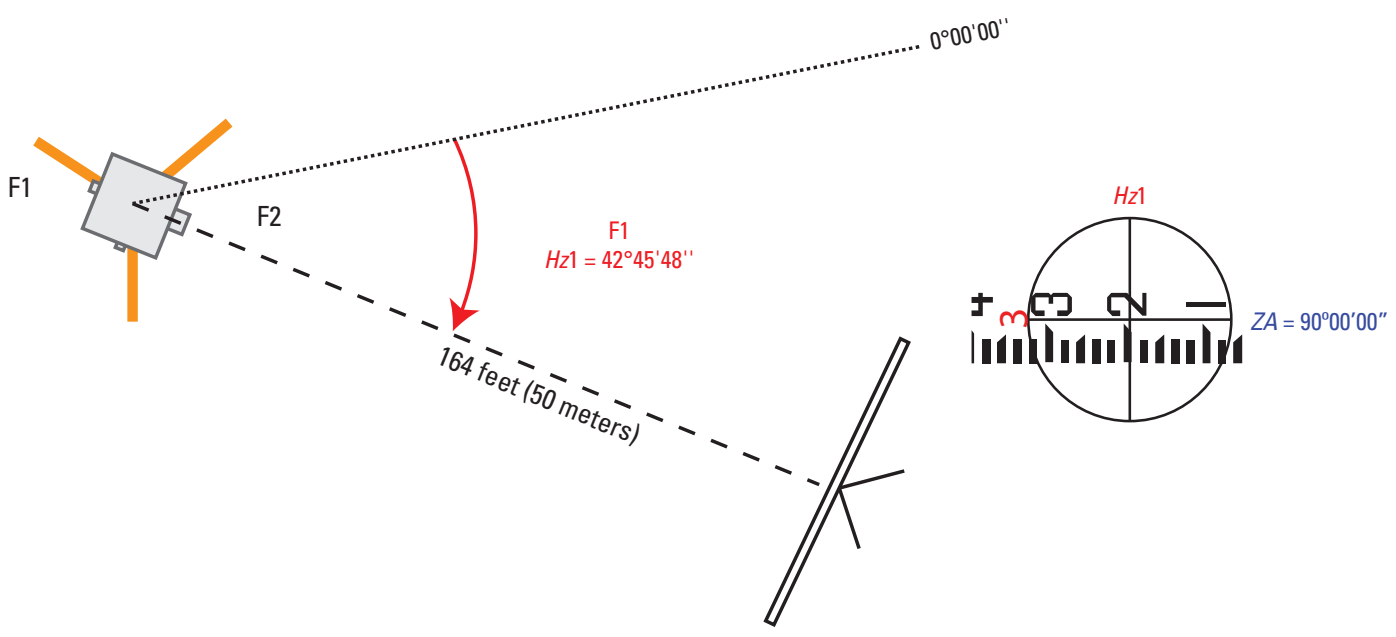

1. Adjust the vertical circle reading of the total station for vertical collimation error using the previously described procedure.

2. Set up the total station. Ensure the instrument is level.

3. Set up a calibrated rod or staff horizontal to the ground about 164 feet (50 meters) from the total station and make sure that is level and aligned with the horizontal plane of the total station.

4. In direct instrument orientation (F1), point the instrument at the rod and take a reading where the vertical crosshair of the telescope aligns with a rod increment. Make sure the zenith angle $(Z A)$ is reading 90 degrees when the telescope is pointed at the rod. Record the horizontal angle $(H z 1)$. An example of the horizontal angle reading is $42^{\circ} 45^{\prime} 48^{\prime \prime}$. The rod reading for this example is 3.19 feet.

B

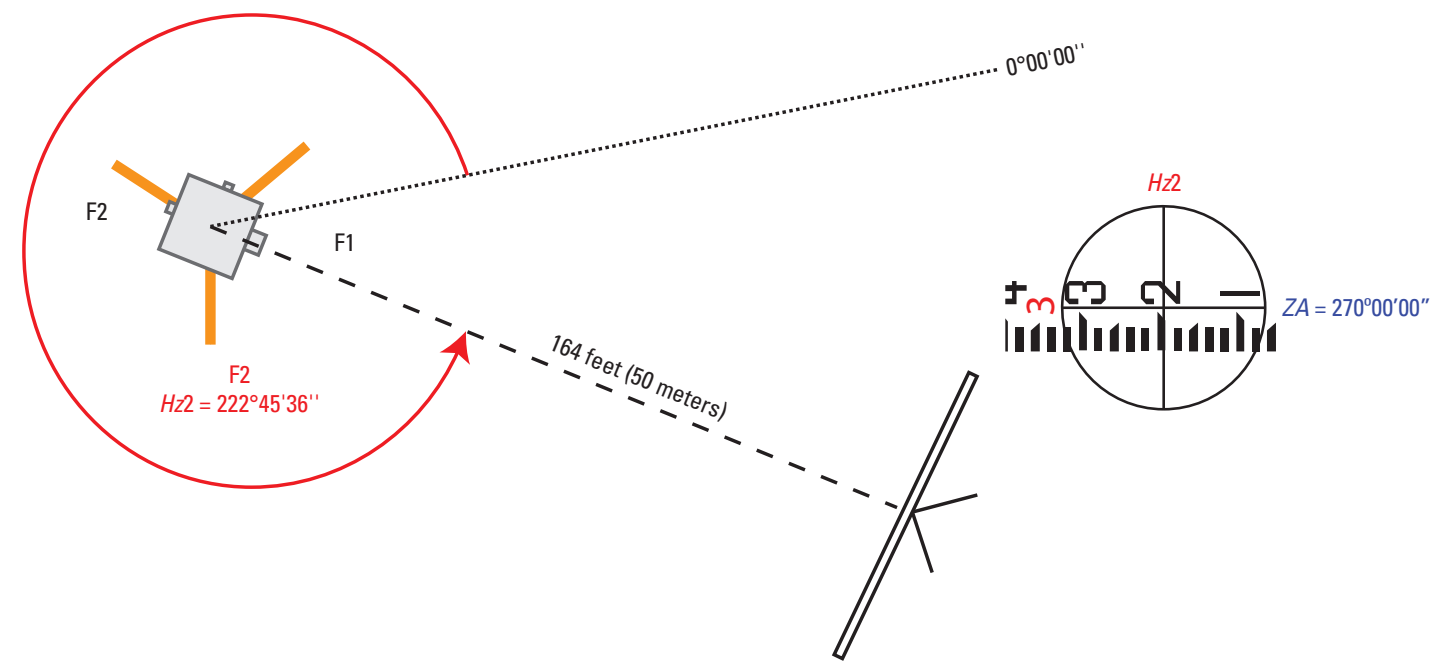

5. In reverse instrument orientation (F2), point the instrument at the rod increment from step 4 (3.19 feet for this example) and record the horizontal angle ( $\mathrm{Hz2}$ ). Make sure the zenith angle is reading 270 degrees. An example of the horizontal angle reading is $222^{\circ} 45^{\prime} 36^{\prime \prime}$.

Calculate the horizontal collimation error (c) using the following formula: $C=\underline{H z 1-\left(H z 2-180^{\circ}\right)}$

\section{EXPLANATION}

\section{- $\quad$ - Line of sight from total station to calibrated staff \\ Arbitrary reference line where horizontal angle reading of total station is $0^{\circ} 00^{\prime} 00^{\prime \prime}$}

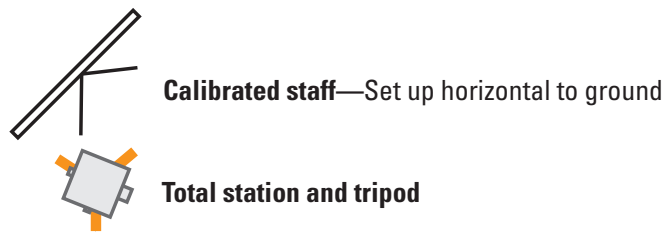

Figure 11. Method for determining horizontal collimation error of a total station. $A$, horizontal angle measurement from total station to rod in direct instrument orientation; and $B$, horizontal angle measurement from total station to rod is reverse instrument orientation. 
the resulting linear horizontal uncertainty $(X)$ of the measurement caused by horizontal collimation error would be greater than $\pm 0.1 \mathrm{ft}$. If previously surveyed locations are remeasured using a stake-out program (Noll and Chu, 2017), a horizontal uncertainty of about $\pm 0.1 \mathrm{ft}$ for a slope with a downslope angle of $50^{\circ}$ can potentially cause an elevation uncertainty $(Z)$ of about $\pm 0.09 \mathrm{ft}$ (fig. 12):

$$
\begin{gathered}
\tan \left(C_{z}\right)=X \div d \\
\tan \left(42^{\prime \prime} \div 3600^{\prime \prime}\right)=X \div 500 \mathrm{ft} \\
\tan (0.0117)=X \div 500 \mathrm{ft} \\
X=\tan (0.0117) \times 500 \mathrm{ft} \\
X= \pm 0.102 \mathrm{ft} ; \text { and } \\
\tan (m)=X \div Z \\
\tan \left(50^{\circ}\right)=0.102 \mathrm{ft} \div Z \\
1.1918=0.102 \mathrm{ft} \div Z \\
Z=0.102 \div 1.1918 \\
Z= \pm 0.086,
\end{gathered}
$$

where

$\begin{array}{ll}m & \text { is the slope, in degrees; } \\ X & \text { is the horizontal uncertainty, in feet; and } \\ Z & \text { is the elevation uncertainty, in feet. }\end{array}$

\section{Tilting-Axis Error}

Tilting-axis error is a deviation from the perpendicular alignment of the horizontal axis and the vertical axis (fig. $9 C$; Leica Geosystems, 2013). This error is an axial defect that varies with the tangent of the zenith angle and only affects the uncertainty of the horizontal angle for steep sightings $\left(45^{\circ}>\right.$ zenith angle $\left.>135^{\circ}\right)$. The tilting-axis error can be determined using the following procedure (fig. 13):

1. Ensure the vertical circle has been adjusted for vertical and horizontal collimation error using the previously described procedures.

2. Set up the total station and ensure it is level. Select an elevated location with a clear line of sight $30^{\circ}$ above and below the horizontal plane of the instrument. Steep zenith angles are required for a good result.

3. Set (or select) an elevated survey target approximately $30^{\circ}$ above the horizontal plane of the total station and approximately $164 \mathrm{ft}(50 \mathrm{~m})$ from the total station.
4. Set up a calibrated rod directly below the elevated target and horizontal to the ground. The rod should be approximately $30^{\circ}$ below the horizontal plane of the total station. Ensure the rod is level.

5. In F1, point the instrument at the elevated target and lock the horizontal tangent screw.

6. In F1, vertically rotate the instrument approximately $30^{\circ}$ below the horizontal plane and take a reading on the horizontal rod where the vertical crosshair of the telescope aligns with a rod increment.

7. Reverse the scope and point the instrument (F2) at the elevated target and lock the horizontal tangent screw.

8. In F2, vertically rotate the instrument $30^{\circ}$ below the horizontal plane and take a reading on the horizontal rod. Determine the difference between the values observed on the calibrated rod in step 6 and step 8 and divide it by two - this value is the magnitude of tilting-axis error for a zenith angle of $120^{\circ}$. The error for this example is $0.025 \mathrm{ft}$.

9. Solve for the VD between the horizontal planes of the elevated target and the calibrated rod by

$$
V D=\tan \left(30^{\circ}\right) \times 164 \mathrm{ft}=94.7 \mathrm{ft}
$$

$$
94.7 \mathrm{ft} \times 2=189.4 \mathrm{ft}
$$

10. Solve for the tilting-axis error at a zenith angle of $120^{\circ}$ $\left(T_{120}\right)$ by

$$
\begin{gathered}
T_{120}=\arctan (0.025 \div 189.4) \\
T_{120}=0.0075628^{\circ} \\
T_{120}=27.2^{\prime \prime}
\end{gathered}
$$

The titling-axis error $(t)$, which can be used to determine the tilting-axis error for any zenith angle $\left(T_{z}\right)$, can be calculated by the following equation using the error determined in figure 13 as an example:

$$
\begin{gathered}
t=T_{z} \times \tan (z) \\
t=0.0075628^{\circ} \times \tan \left(120^{\circ}\right) \\
t=-0.0131^{\circ}
\end{gathered}
$$

where

$t \quad$ is the defect, or tilting-axis error, in degrees;

$T_{z} \quad$ is the tilting-axis error for a zenith angle, in degrees, and

$z \quad$ is the zenith angle, in degrees. 


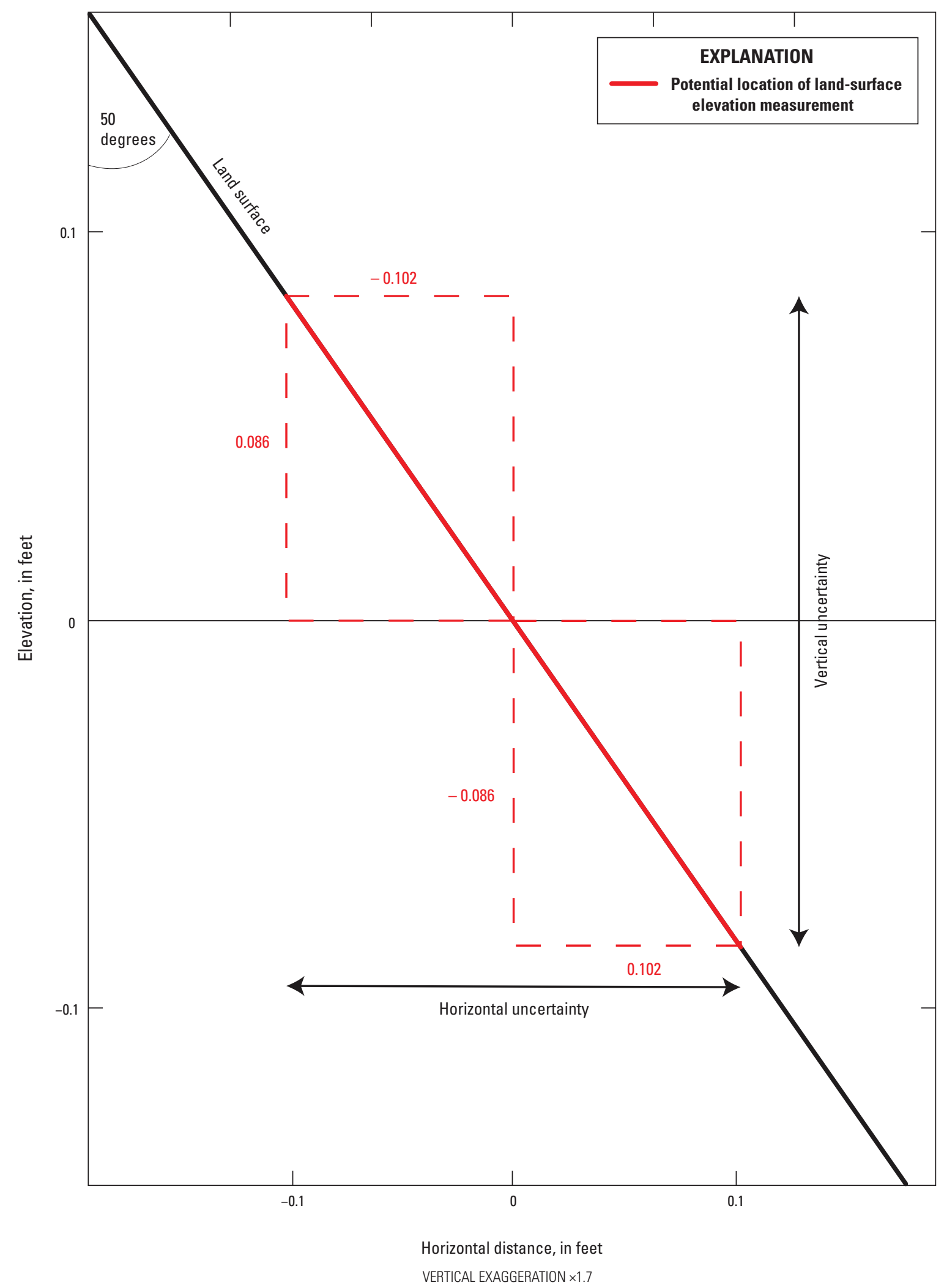

Figure 12. Potential elevation error resulting from a horizontal positional error of plus or minus 0.102 feet on a slope with a downslope angle of about 50 degrees. Vertical and horizontal datum are assumed. 
$\boldsymbol{A}$

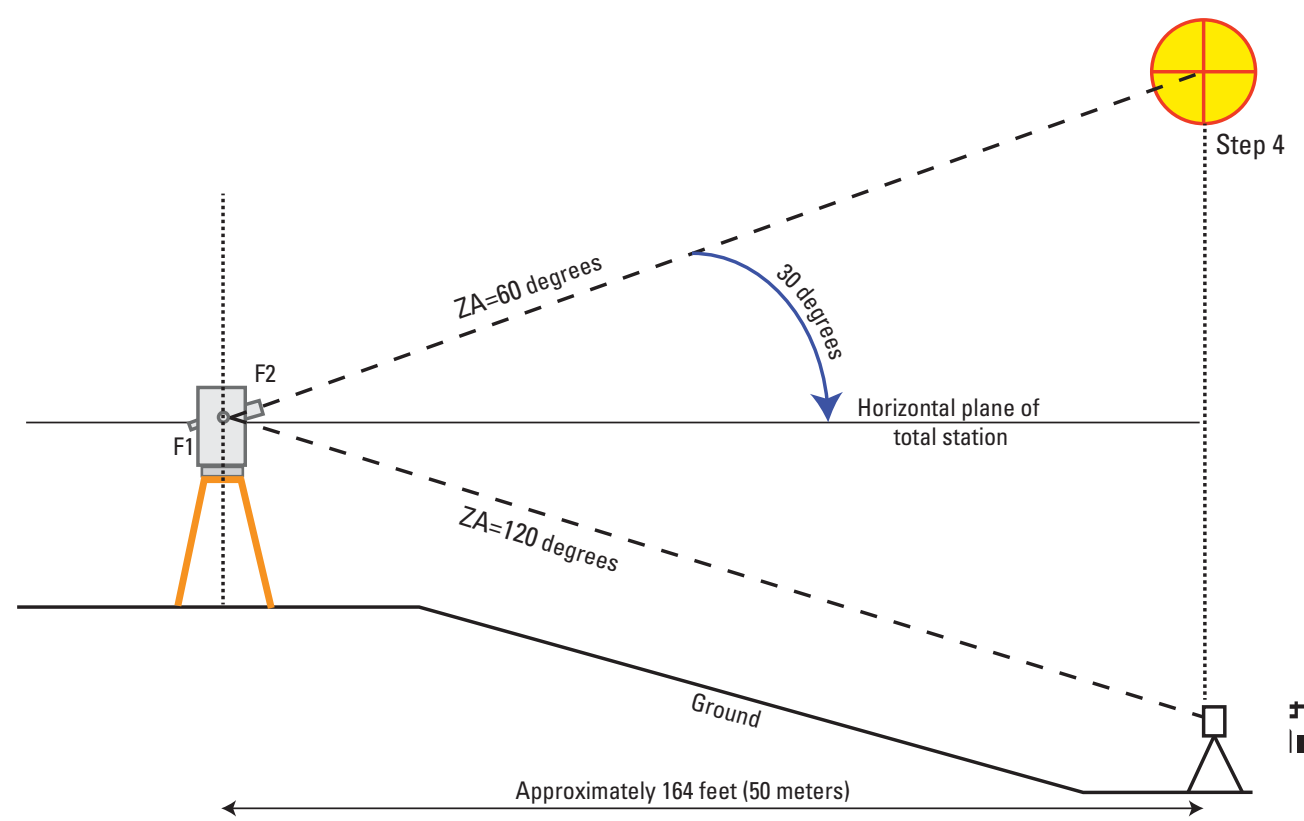

Step 6

F1 rod reading $=3.14$ feet

Make sure the vertical circle has been adjusted for vertical and horizontal collimation error using the previously described procedures.

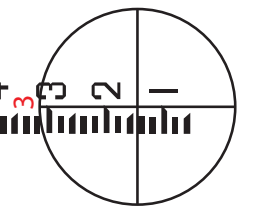

2. Set up the total station and ensure it is level. Select an elevated location with a clear line of sight 30 degrees above and below the horizontal plane of the instrument. Steep zenith angles are required for a good result.

3. Set (or select) an elevated survey target approximately 30 degrees above the horizontal plane of the total station and approximately 50 meters (164 feet) from the total station.

4. Set up a calibrated rod directly below the elevated target and horizontal to the ground. The rod should be approximately 30 degrees below the horizontal plane of the total station. Make sure the rod is level.

5. In direct instrument orientation (F1), point the instrument at the elevated target and lock the horizontal tangent screw.

6. In F1, vertically rotate the instrument approximately 30 degrees below the horizontal plane and take a reading on the horizontal rod where the vertical crosshair of

$\boldsymbol{B}$ the telescope aligns with a rod increment.

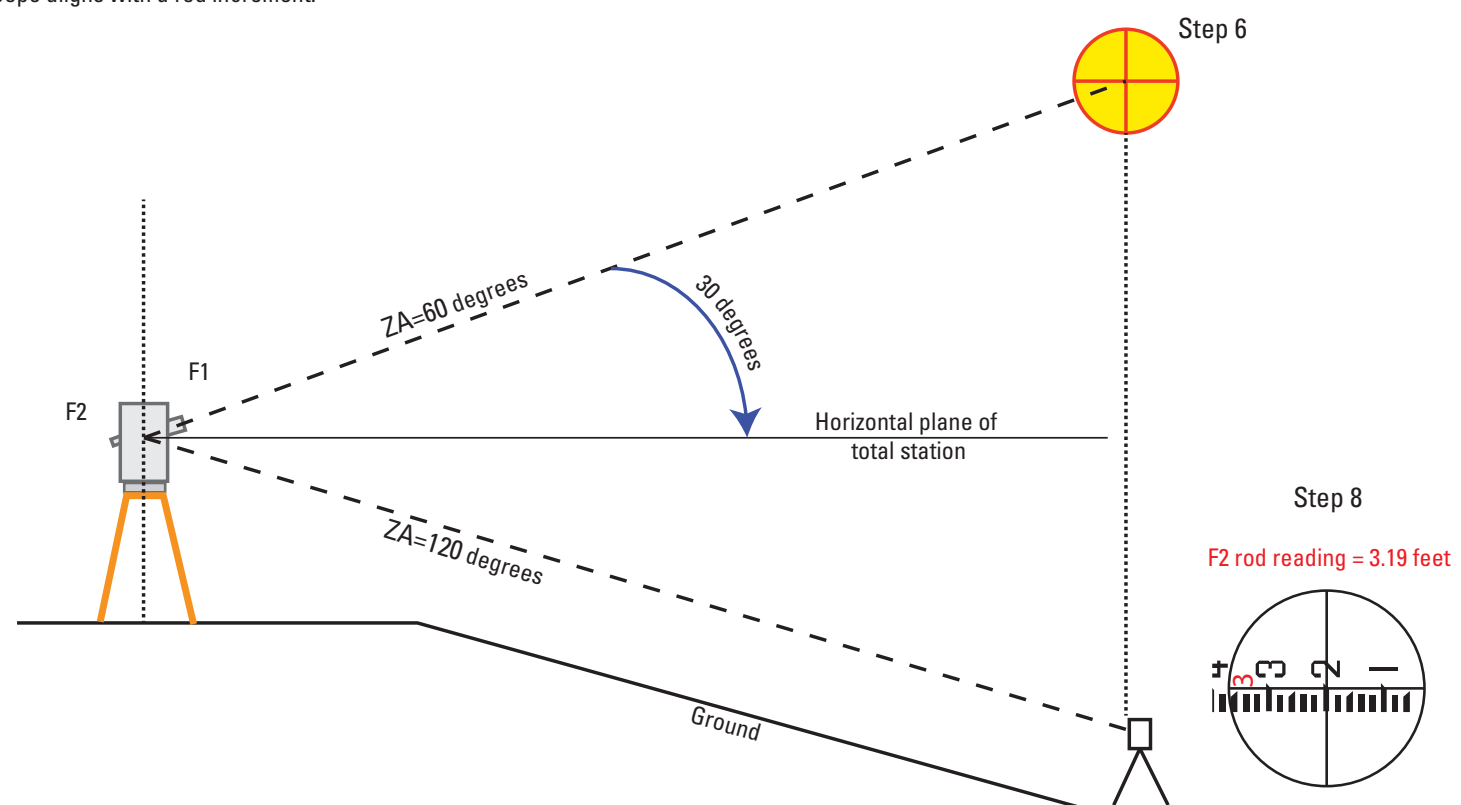

7. Reverse the scope and point the instrument (reverse instrument orientation [F2]) at the elevated target and lock the horizontal tangent screw.

8. In F2, vertically rotate the instrument 30 degrees below the horizontal plane and take a reading on the horizontal rod. Determine the difference between the values observed on the calibrated rod in step 6 and step 8 and divide it by two - this value is the magnitude of tilting-axis error for a zenith angle of 60 degrees (or 120 degrees).

The error for this example is 0.025 feet

\section{EXPLANATION}

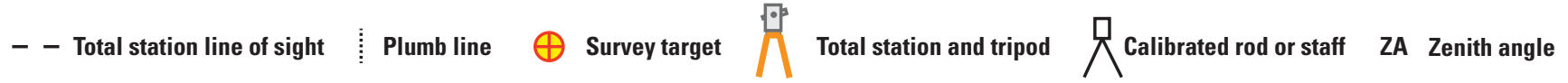

Figure 13. Method for determining tilting-axis error of a total station. $A$, steps 1-6. B, steps 7-8. C, steps 9-10. 


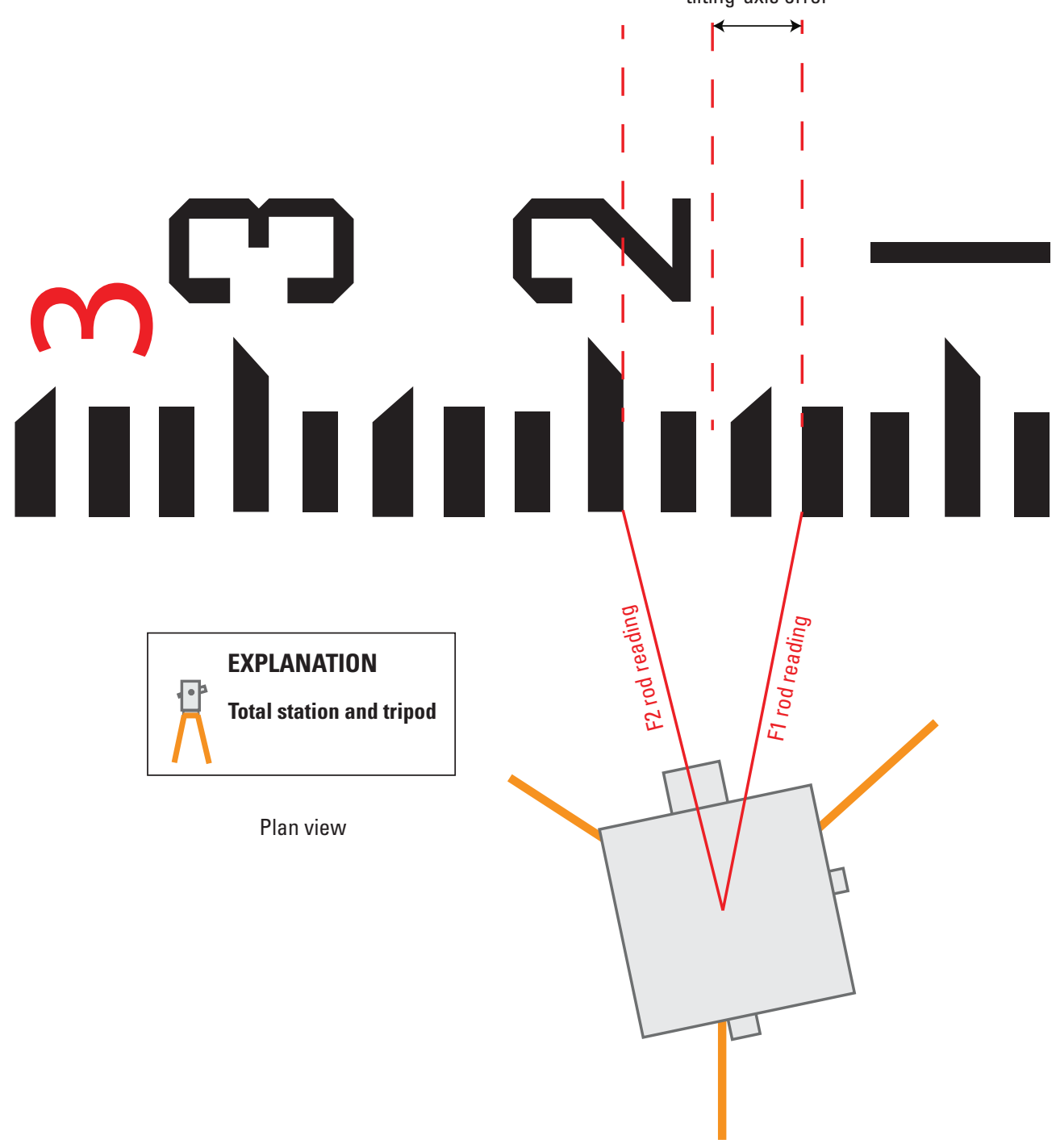

9) Solve for the vertical difference $(V D)$ between the horizontal planes of the elevated target and the calibrated rod by $V D=\tan (30$ degrees $) \times 164$ feet $=94.7$ feet 94.7 feet $\times 2=189.4$ feet

10) Solve for the tilting-axis error at a zenith angle of 120 degrees $\left(T_{120}\right)$ by

$T_{120}=\arctan (0.025 \div 189.4)$

$T_{120}=0.0075628$ degrees

$T_{120}=27.2$ seconds

Figure 13. Method for determining tilting-axis error of a total station. $A$, steps $1-6$. $B$, steps $7-8$. $C$, steps $9-10$. 
Corrections for vertical and horizontal collimation error, and tilting-axis error can be determined, stored, and applied by most modern total stations to ensure precise field measurements. If a total station does not have this capability, observing in F1 and F2 cancels most of the uncertainty associated with axial defects because the F1 observation has an equal and opposite axial error compared to the $\mathrm{F} 2$ observation (Ogundare, 2016). If these procedures are followed, and the F1 and F2 measurements are averaged, most of the uncertainty associated with axial defects can be reduced or eliminated for instruments that do not have the capacity to store and apply corrections. In any case, it is always good practice to keep an instrument in proper adjustment, but also to use it as if it has not been adjusted (Brinker and Minnick, 1995).

\section{Vertical-Axis Error (Tilt of the Vertical Axis)}

Vertical-axis (standing-axis) error is caused when the vertical axis of the instrument is not aligned with the direction of gravity (figs. 7 and 9D; Leica Geosystems, 2013). Verticalaxis error is not considered an axial defect because it is caused by poor leveling procedures of the instrument operator, by a poorly adjusted compensator, or by both. The error can affect the horizontal and zenith angles and cannot be corrected with F1 and F2 measurements. A good operator can usually align the vertical axis of the total station with the direction of gravity within 5" to 10" (Joseph Paiva, GeoLearn, LLC, written commun., 2019). A dual-axis compensator that is properly adjusted will automatically correct the horizontal and zenith angles when the instrument is not completely level and the telescope is inclined or declined from the horizontal direction (Cothrun, 1995). If the dual-axis compensator is poorly adjusted, the error from the tilt of the vertical axis will persist and reduce the quality of the data. Good leveling procedures and a precisely adjusted dual-axis compensator are critical for reducing measurement uncertainty from this source of error.

Modern technology has eliminated or reduced other sources of error related to axial defects and alignment of the horizontal and vertical circles. Historically, circle graduation error was a major source of angular uncertainty for theodolites and total station instruments (USACE, 2007). Photoetching technology is now used to more precisely mark the graduations on the circles. Any residual errors from graduation eccentricity, as well as errors in mounting the circles (the centers of the graduations should coincide with the vertical and horizontal axes), are removed by evaluating and storing the errors in read-only memory and applying corrections; or by electronically sensing the graduations at diametrically opposite sides of the circle. By averaging the readings at each sensing point, the latter method thus provides accurate circle readings.

\section{Testing the Electronic Distance Measurement Instrument}

Total station instruments are essentially electronic theodolites with an integrated EDMI that is used to measure slope distances. The EDMI measures the slope distance between the instrument and a reflector by transmitting a pulsed or modulated electromagnetic signal that is typically infrared or visible light. The returned signal is then processed by the EDMI and a slope distance is determined using a phase difference, or two-way travel time method, which is described in the "Trigonometric-Leveling Equipment" section. Slope distances are reduced to the horizontal equivalent by multiplying the EDM by the sine of the zenith angle (fig. 8).

The uncertainty of an EDM depends on (1) a constant (fixed) error, which is related to the additive and cyclic (bias) errors associated with surveying instruments; and (2) a distance-weighted (scale) error, which is proportional to the measurement distance (fig. 14). These two components of the EDM error budget, which are summed to determine total EDM uncertainty, are specified by the manufacturer and should be verified to ensure the EDMI is operating properly before a land survey campaign. The EDM constant and distanceweighted uncertainties are typically around $3 \mathrm{~mm}$ and $3 \mathrm{ppm}$, respectively, but range from about $5 \mathrm{~mm}$ and $5 \mathrm{ppm}$ to as low as 1 or $2 \mathrm{~mm}$ and $1 \mathrm{ppm}$. Sources of constant error are related to (1) the uncertainty of the electronic source (origin) of the measurement, which includes (but is not limited to) differences between the electronic center and the mechanical center of the EDMI, differences between the optical and mechanical centers of the reflector, systematic errors such as improper instrument calibration, incorrect accounting of the prism constant, and poor centering of the total station over the survey marker; and (2) the uncertainty of the reflected position (reversal point) of the EDM signal within the prism, which includes systematic errors like improper pointing of the total station (which can affect the quality of the returned signal) and poor centering of the prism over the target location (Ogundare, 2016; Brinker and Minnick, 1995, Rüeger, 1990).

The distance-weighted component of the EDM error budget is proportional to the measured distance. Sources of distance-weighted error include random uncertainty from atmospheric conditions, such as heat shimmer, and systematic error from EDMI components, such as the crystal oscillator, that can be adversely affected by age and atmospheric temperature conditions, which can cause modulation frequencies to drift (Rüeger, 1990; Brinker and Minnick, 1995). Ovencontrolled, temperature-compensated, and noncompensated room temperature crystal oscillators are used to minimize the effects of temperature on modulation frequency; additionally, manufacturers can determine how modulation frequency drifts with temperature and can program the EDMI microprocessor to apply a correction to obtain a temperature-compensated distance measurement (Rüeger, 1990). All constant errors when comprehensively evaluated are also distance weighted. For example, poor centering of the total station above a 


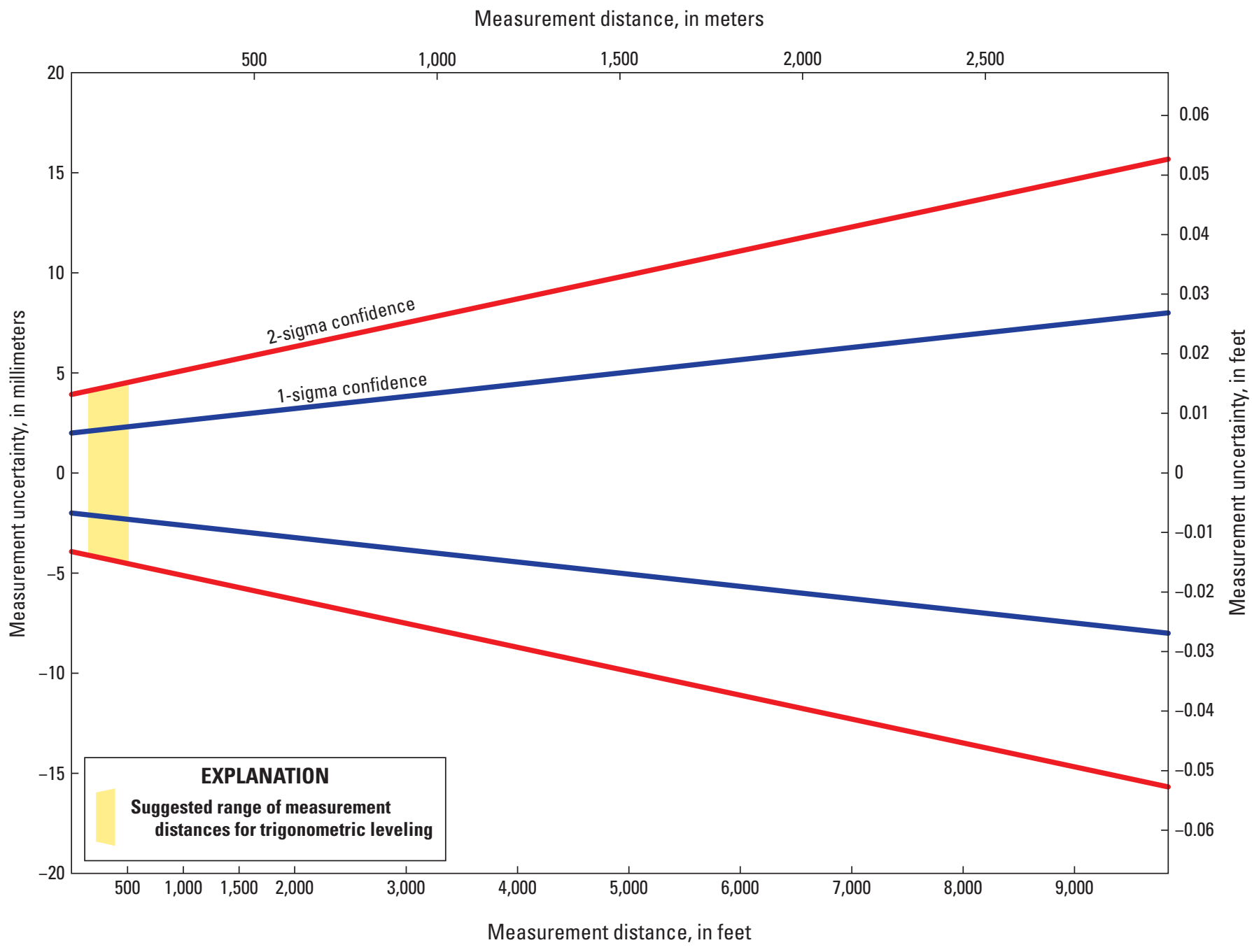

Figure 14. Total uncertainty of an electronic distance measurement for a total station with a constant error of 2 millimeters and a distance-weighted error of 2 parts per million. Modified from Brinker and Minnick (1995).

survey marker is a systematic error that affects the constant error. But that error, when applied to the measurement (that is, the distance), also has distance-weighted implications. One millimeter of centering uncertainty propagated over a 5,000-m (16,404.2-ft) baseline could cause an error of 1 part per 3,500,000 parts (Brinker and Minnick, 1995). Measurement distances of less than $500 \mathrm{ft}$ are recommended for quality trigonometric-leveling measurements to reduce the effects of distance-weighted sources of error; conversely, for small measurement distances (less than $150 \mathrm{ft}$ ), the constant error (if large enough) can become a significant part of the error budget.

Additional sources of uncertainty include random errors such as inaccurate accounting of atmospheric conditions such as air temperature and barometric pressure, which are used to correct the EDM for atmospheric refraction. For example, an error of 1 degree Celsius $\left({ }^{\circ} \mathrm{C} ; 1.8^{\circ} \mathrm{F}\right)$ could cause a distanceweighted error of $0.8 \mathrm{ppm}$ for infrared distance measurements
(USACE, 2007). Quality meteorological instruments (barometers and thermometers) that are calibrated against a known standard must be used to accurately measure ambient temperature and barometric pressure conditions in the field before a land survey campaign and should be carefully accounted for in the data-collection device so that corrections can be automatically applied. Although it is unlikely that meteorological conditions would substantially vary over a $500-\mathrm{ft}$ measurement distance, it is recommended that air temperature and barometric pressure measurements are made at the station (instrument) and foresight (prism) locations; relative humidity, wind speed, cloud cover, and visibility should also be measured and noted. These meteorological measurements (atmospheric pressure and ambient temperature) should be entered into the data-collection device to correct for the effects of atmospheric refraction. Corrections for the curvature of the Earth and refraction of the transmitted signal by the atmosphere over long baseline distances should also be accounted for 
before EDMI testing. This is done by ensuring that the correct parameters have been set in the data-collection device to automatically correct for these errors. Measurement uncertainty caused by curvature and refraction, additional sources of EDM uncertainty, and other error sources associated with total station measurements and trigonometric leveling are described in the "Sources of Measurement Uncertainty for Trigonometric Leveling" section.

The EDMI must be routinely tested to verify (or determine) constant and distance-weighted sources of measurement uncertainty. Calibrated baselines are used to ensure the EDMI is meeting the manufacturer specification for distance measurements and to determine and monitor instrument accuracy and precision (repeatability) over time in field conditions (NOAA, 2017b). The National Geodetic Survey cooperatively maintains a network of about 400 calibrated baselines across the country for this purpose (fig. 15). These highly accurate baselines are accessible to surveyors in each State and provide a national standard for distance measurement. A standard calibration baseline consists of four to six stable monuments that are distributed in a straight line over about $1,000 \mathrm{~m}$
$(3,280.84 \mathrm{ft})$ or longer. Distances are initially measured with calibrated instrumentation that is accurate to one order of magnitude greater than the instrument that will be calibrated over the baseline. Calibrated baselines are subdivided into two categories (tier 1 and tier 2), which indicates whether NGS personnel established the baseline or a cooperating agency or partner. Tier 1 baselines are established by the NGS, or under direct supervision by the NGS, using NGS-owned equipment. Tier 2 baselines are established by a partner, or other cooperating agency, under the direct supervision of the NGS with cooperator-owned equipment. Calibrated baselines are established and used according to the criteria described in the NOAA technical memorandum NOS NGS 8 (Dracup and others, 2019) and NOS NGS 10 (Fronczek, 1977). In 2017, changes within the NGS and new technology prompted changes in baseline policy and procedures; currently, the NGS maintains only one calibrated baseline per state but also manages a database of calibrated baselines that is available to the public (Dracup and others, 2019).

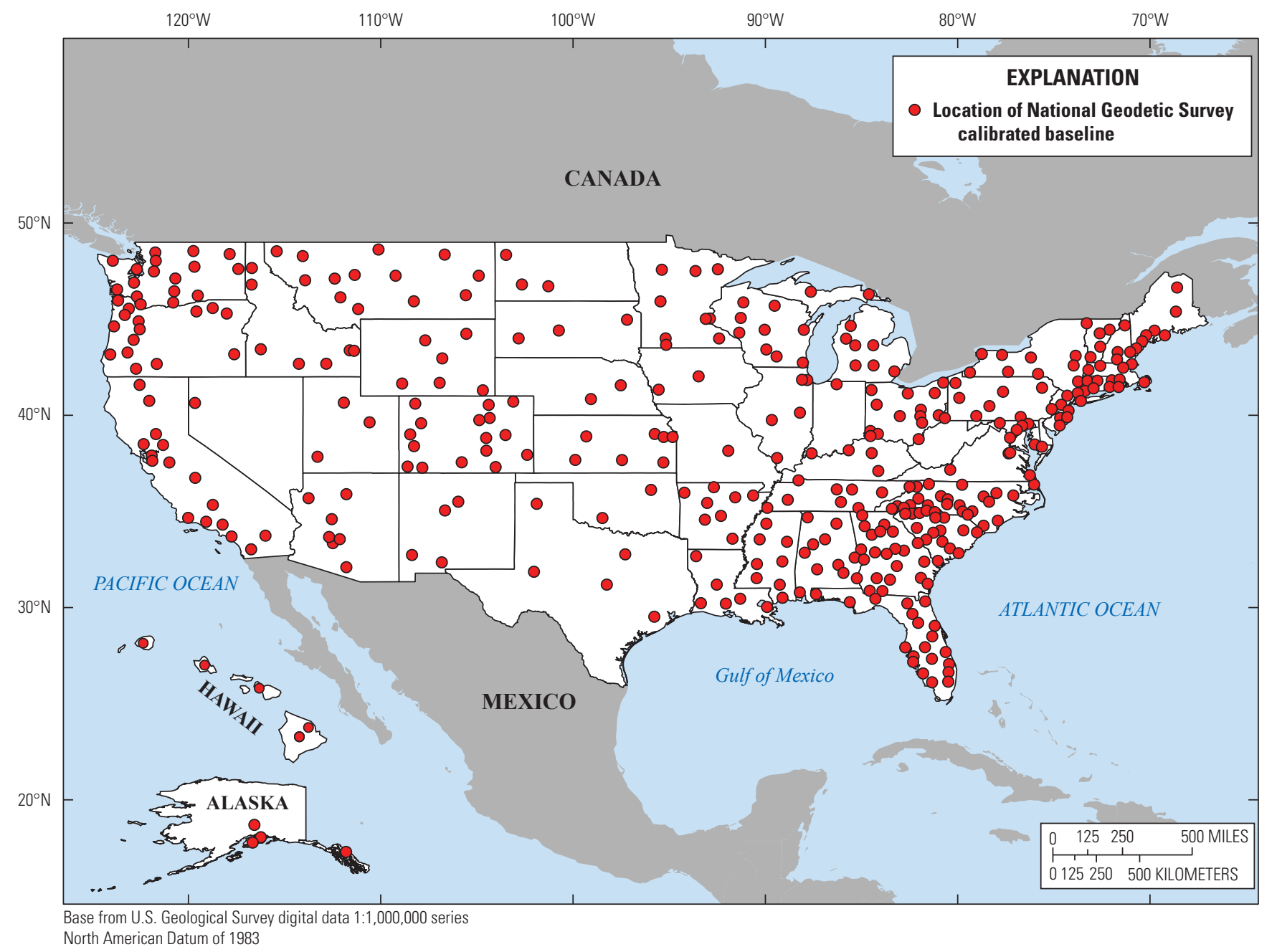

Figure 15. Location of National Geodetic Survey calibrated baselines. 
Before EDMI testing, the surveyor should be fully familiar with the total station operations manual and make sure all the equipment is in good working order according to the manufacturer specification. Adjustable tripods with a dual clamp locking mechanism, quality retroreflectors with known offsets, and precisely adjusted tribrachs are recommended for trigonometric-leveling field campaigns and should also be used during EDMI testing to ensure the EDMI and accessory equipment are operating properly. Forced-centering devices such as tripod-mounted tribrachs and tripods with a center pole may also be used to ensure proper alignment over the survey marker.

\section{Electronic Distance Measurement Instrument Testing Procedure at National Geodetic Survey Calibrated Baselines}

Total stations with integrated EDMIs should be periodically tested on calibrated baselines at dedicated facilities at least once a year or more frequently when conditions warrant. Some examples of such conditions that require EDMI testing besides the recommended annual testing are (1) when the constant and scale errors of the EDMI need to be determined or verified, (2) if the instrument was potentially damaged during transport or field use, (3) when using older instruments that need to be checked more frequently to ensure they are still operating within the manufacturer specifications, and (4) before survey control projects that require EDMI testing before field work (Ogundare, 2016). If EDMI tests indicate the instrument is operating outside of the manufacturer specified uncertainty, the instrument must be sent back to the manufacturer for calibration. In some cases, the operation manual for the total station may provide a procedure to determine the instrument constant or enter adjustments that correct the distance measurement, such as adjusting the modulation frequency of the EDMI. However, it is recommended that adjustments to the EDMI are done in facilities authorized by the manufacturer. Preparation for the EDMI testing procedure is as follows:

1. Identify the NGS calibrated baseline(s) in your State by accessing the NGS website (https://geodesy.noaa.gov/ CBLINES/index.shtml).

2. Identify the manufacturer-specified constant and distance-weighted error for the integrated EDMI of the total station.

3. Check weather to ensure that conditions are within the manufacturer-specified range for EDMs.

4. Ensure all equipment needed for EDMI testing is in good operating condition and the team preparing to perform the testing process is familiar with the operations manual. Equipment includes (but is not limited to) the total station with a quality tribrach and precisely adjusted optical or laser plummet; adjustable tripod with dual-clamp locking mechanism or tripod with adjust- able center pole with fixed-height dowel stops (for total station and prism assembly); quality retroreflector with known offset and tribrach; fixed ruler; and calibrated meteorological instruments such as a barometer and thermometer, field book, and pens. An external datacollection device is not required if the integrated microprocessor on the total station has the capability to correct measurements for curvature and refraction.

An active NGS calibrated baseline is defined by the NGS as a highly accurate baseline that is freely accessible to the public (although in some locations you may need to contact the site owner/manager for permission to enter the property on which the baseline is located) as a standard for length measurement and for which all associated marks and their adjusted distances have not been identified as unusable or unreliable (NOAA, 2017b). For each state, NGS provides users with location descriptions and the adjusted results of calibration baseline measurements. Once an active NGS calibrated baseline has been identified, weather conditions are suitable, and the surveying equipment is in good operating condition, the following procedure should be used to test the EDMI. The following example uses the calibrated baseline in Hempstead, New York (fig. 16).

The Hempstead calibrated baseline consists of five plain monumented brass disks (survey markers) that are distributed at variable distances along a 1,044.5262-m (3,426.923-ft) line that runs approximately north and south at Eisenhower County Park (not shown), in Hempstead, N.Y. (fig. 16). Starting at the 0 North Base survey marker, a combination of 10 independent measurements of varying distances can be made along the calibrated baseline by setting up the total station above each monument once, except the South Base 1,044 survey marker. The starting position of the retroreflector is always the first survey marker south of the instrument and is moved incrementally south over each monument after each distance measurement is made, until the final position at the 1,044 South Base survey marker. The procedure is as follows:

1. Set up the total station above one of the endpoints of the baseline. Ensure the instrument is level and centered over the survey marker. For the Hempstead baseline, this is 0 North Base monumented brass disk.

2. Set up the tripod with prism assembly above the first survey marker (122 P.O.L. 3 for Hempstead) along the calibrated baseline that is closest to the instrument. Ensure the prism is level and centered over the survey marker.

3. Account for refraction. Make meteorological observations (air temperature, barometric pressure, relative humidity, wind speed, cloud cover, and visibility) at the total station and prism location with a calibrated instrument. Calculate an average observation if there is a substantial temperature or pressure difference between the station (total station) and target (prism) locations. Record the observations in the field book, then enter the observations into the data-collection device so that refraction 


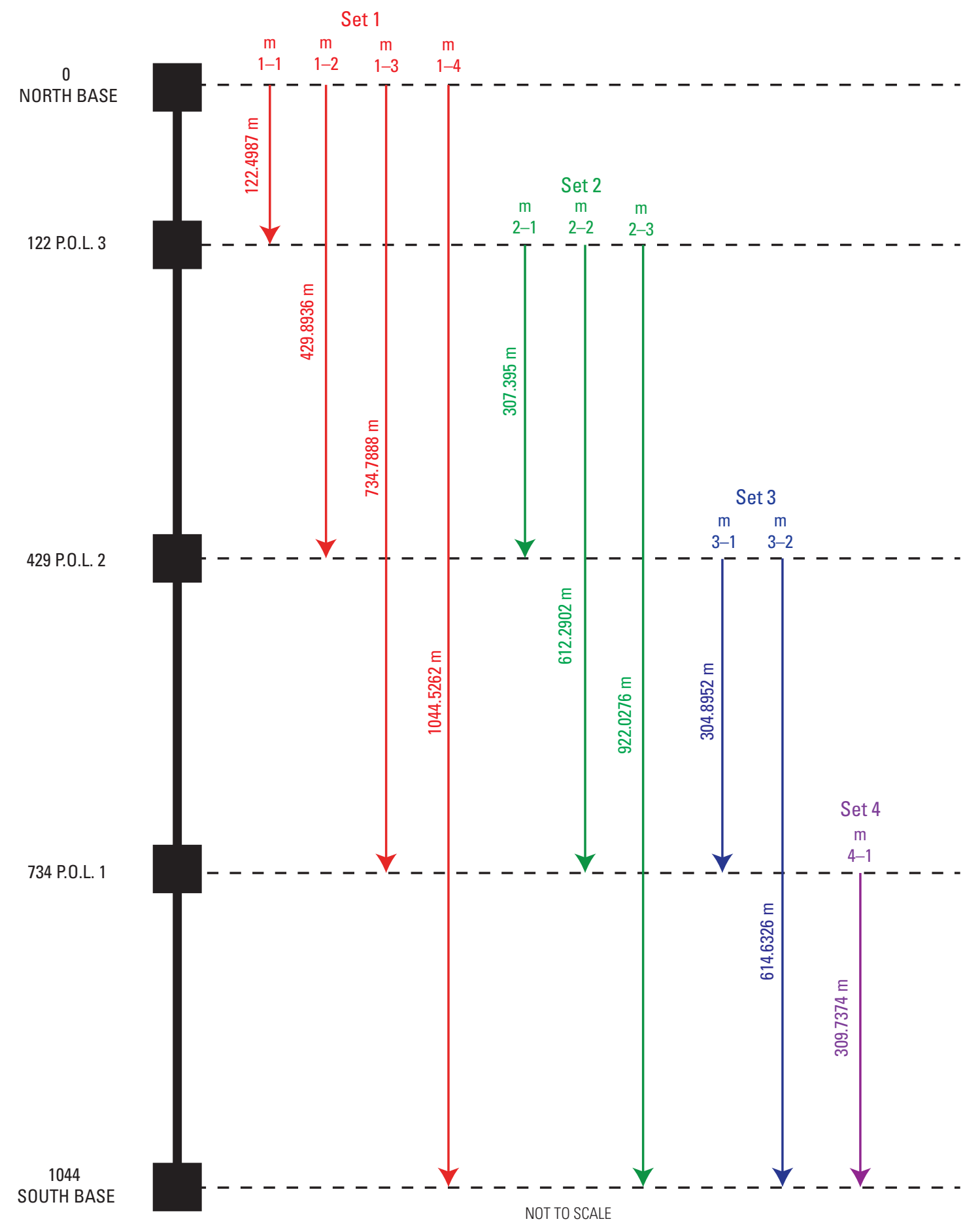

EXPLANATION

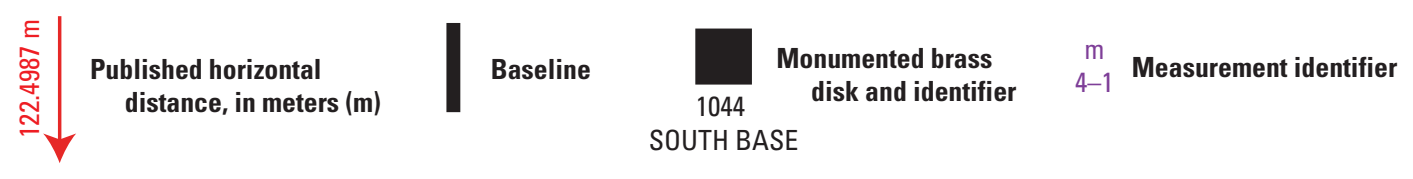

Figure 16. National Geodetic Survey calibrated baseline configuration at Hemsptead, New York. One of the calibrated baselines shown in figure 15 . 
corrections are applied to the distance measurement. Keeping a record of important field observations in a field book is a crucial quality-assurance procedure.

4. Account for curvature. Ensure the data-collection device is applying a correction for the curvature of the Earth for the indicated baseline distance.

5. Precisely point the instrument at the prism located above 122 P.O.L. 3 and take the first slope measurement as an F1 observation. Record the slope distance, zenith angle, and derived horizontal distance. An example horizontal distance and zenith angle for the Hempstead baseline measurement $\mathrm{m} 1-1$ is $122.4980 \mathrm{~m}(401.8963 \mathrm{ft})$ and $90^{\circ} 14^{\prime} 24^{\prime \prime}$, respectively.

6. Reverse the scope and take the second slope measurement in F2. Record the slope distance, zenith angle, and derived horizontal distance. An example m1-1 F2 measurement is $122.4984 \mathrm{~m}(401.8976 \mathrm{ft})$ and $269^{\circ} 45^{\prime} 48^{\prime \prime}$.

7. Add F1 zenith angle observation to $\mathrm{F} 2$ observation and subtract $360^{\circ}:\left(\left[90^{\circ} 14^{\prime} 24^{\prime \prime}+269^{\circ} 45^{\prime} 48^{\prime \prime}\right]-360^{\circ}\right)$ $=00^{\circ} 00^{\prime} 12^{\prime \prime}$. Divide the difference by 2 , as follows: $00^{\circ} 00^{\prime} 12^{\prime \prime} \div 2=00^{\circ} 00^{\prime} 06^{\prime \prime}$.

8. A quotient that substantially exceeds the angular uncertainty of the instrument may indicate a pointing error or poor collimation. If this occurs during EDMI testing, reference the vertical collimation procedure section in this manual to adjust the instrument. If a pointing error is indicated, observe a second set of F1 and F2 measurements.

9. If the quotient does not substantially exceed the angular uncertainty of the instrument, average the derived horizontal distances from the F1 and F2 observations. The total station display can be toggled to show the horizontal equivalent of the reduced slope distance. The hypothetical angular uncertainty of the instrument being tested on the Hempstead baseline is 5", so the horizontal distances can be averaged. For $\mathrm{m} 1-1$, the average hori- zontal distance is $122.4982 \mathrm{~m}$ (401.897 ft). Multiple sets of F1 and F2 observations should be made and averaged to quality ensure the derived horizonal distance.

10. Subtract the measured distance from the known distance for measurement $\mathrm{m} 1-1$. The difference between the known $(122.4987 \mathrm{~m})$ and the observed horizontal distance measurement $(122.4982 \mathrm{~m}$ ) for $\mathrm{m} 1-1$ is $0.0005 \mathrm{~m}(0.0016 \mathrm{ft})$.

11. Calculate the manufacturer specified total uncertainty (constant plus distance-weighted error) for the EDMI tested on the indicated baseline. For this EDMI, the constant and distance-weighted error is $2 \mathrm{~mm}$ and $2 \mathrm{ppm}$, respectively. The total manufacturer EDM uncertainty for an EDM of $122.4982 \mathrm{~m}$ is $2 \mathrm{~mm}+122,498.2 \mathrm{~mm} \times(2 \div$ $1,000,000)=2.25 \mathrm{~mm}$.

12. Compare the manufacturer specified total EDM uncertainty $(2.25 \mathrm{~mm})$ to the measured uncertainty $(0.5 \mathrm{~mm})$ for the baseline of $122.4982 \mathrm{~m}$ for measurement $\mathrm{m} 1-1$. For this measurement, the integrated EDMI is outperforming the manufacturer specification (usually shown as 1 sigma).

13. Repeat the process for the nine other baseline measurements or sets 1 through 4.

14. Calculate the 2-sigma error by multiplying the manufacturer specified total error by 1.96 . If the uncertainty from any of the 10 baseline measurements exceeds the 2-sigma manufacturer-specified uncertainty, send the EDMI for factory calibration.

The data-collection device can be programmed to display the derived horizontal distance for comparison to the known horizontal distance indicated on the NGS datasheet (fig. 17). Mark-to-mark distances indicated on the datasheet can be disregarded because they represent horizontal spatial chord distance between the centers of the disks (Fronczek, 1977). F1 and F2 measurements and accurate accounting of zenith angles are recommended to ensure the instrument is properly collimated for the EDMI testing procedure. 
DESCRIPTION OF HEMPSTEAD BASE LINE

YEAR MEASURED: 1997

LATITUDE: 404418

LONGITUDE: 0733500

AZIMUTH: 341 DEGREES TRUE NORTH

CHIEF OF PARTY: KDS

THE BASE LINE IS LOCATED ABOUT 4 KM (2.5 MI) EAST OF HEMPSTEAD, NY AT THE EISENHOWER COUNTY PARK. IT IS 1 KM (0.5 MI) WEST OF SALISBURY, NY, 3 KM (2.0 MI) NORTH OF UNIONDALE, NY, ON LONG ISLAND, AND 4.5 KM (3.0 MI) SOUTH OF I-495.

THE BASE LINE IS A NORTH-SOUTH LINE WITH THE 0-METER POINT ON THE NORTH END. IT CONSISTS OF THE 0, 122, 429, 734 AND 1044 METER POINTS. THERE IS NO 100 FT. TAPE CALIBRATION MARK.

TO REACH THE 0-METER POINT FROM THE WEST SIDE OF EISENHOWER COUNTY PARK, STEWART AVENUE ENTRANCE, LOCATED ON MERRICK AVENUE BETWEEN HEMPSTEAD TURNPIKE AND OLD COUNTY ROAD, GO EAST ON PARK BOULEVARD FOR $30 \mathrm{M}$ (100 FT), THEN TURN NORTH ONTO THE GRASS. PROCEED NORTH NORTHWEST FOR $150 \mathrm{M}(492 \mathrm{FT})$ TO THE 1044 METER POINT, SOUTH BASE, THEN NORTH ALONG THE LINE OF CBL STATIONS TO THE 0 METER POINT, NORTH BASE, JUST SOUTH OF THE FENCE BOUNDING THE NORTH SIDE OF THE PARK.

THE 0-METER POINT IS A PLAIN BRASS 8 CM DISK STAMPED "NORTH BASE" SET IN THE TOP OF A 50 CM (20 IN) DIAMETER CONCRETE POST RECESSED $10 \mathrm{CM}(4 \mathrm{IN})$ BELOW THE SURFACE OF THE GROUND. IT IS $11.1 \mathrm{M}(36.4 \mathrm{FT})$ NORTHEAST OF THE NORTH FENCE POST OF THE GATE, $7.1 \mathrm{M}$ (23.3 FT) SOUTHEAST OF THE NORTHWEST CORNER OF THE 4 FOOT CHAIN LINK FENCE, AND 4.1 M (13.5 FT) SOUTHWEST OF A 15 CM (6 IN) DIAMETER OAK TREE.

THE 122-METER POINT IS A PLAIN BRASS 8 CM DISK STAMPED "P.O.L. \#3" SET IN THE TOP OF A 50 CM (20 IN) DIAMETER CONCRETE POST RECESSED $10 \mathrm{CM}$ (4 IN) BELOW THE SURFACE OF THE GROUND. IT IS 78.4 M (257.2 FT) WEST OF THE ASPHALT SERVICE ROAD AND 26.3 M (86.3 FT) EAST OF THE 4 FOOT CHAIN LINK FENCE.

THE 429-METER POINT IS A PLAIN BRASS 8 CM DISK STAMPED "P.O.L. \#2" SET IN THE TOP OF A 50 CM (20 IN) DIAMETER CONCRETE POST RECESSED 10 CM (4 IN) BELOW THE SURFACE OF THE GROUND. IT IS 77.6 M (254.6 FT) WEST OF THE ASPHALT SERVICE ROAD AND 26.3 M (86.3 FT) EAST OF THE 4 FOOT CHAIN LINK FENCE.

THE 734-METER POINT IS A PLAIN BRASS 8 CM DISK STAMPED "P.O.L. \#1" SET IN THE TOP OF A 50 CM (20 IN) DIAMETER CONCRETE POST RECESSED 10 CM (4 IN) BELOW THE SURFACE OF THE GROUND. IT IS 30.4 M (99.7 FT) NORTH OF THE ASPHALT SERVICE ROAD, 23.4 M (76.8 FT) EAST OF THE 4 FOOT CHAIN LINK FENCE, AND 11.8 M (38.7 FT) SOUTHWEST OF A 25 CM (10 IN) DIAMETER OAK TREE.

THE 1044-METER POINT IS A PLAIN BRASS 8 CM DISK STAMPED "SOUTH BASE" SET IN THE TOP OF A 50 CM (20 IN) DIAMETER CONCRETE POST RECESSED $10 \mathrm{CM}$ (4 IN) BELOW THE SURFACE OF THE GROUND. IT IS $153.4 \mathrm{M}(503.2 \mathrm{FT})$ NORTH OF THE NORTH BACK OF CURB OF PARK DRIVE, $28.0 \mathrm{M}(91.9 \mathrm{FT})$ NORTHEAST OF A TREE, $26.0 \mathrm{M}$ (85.3 FT) EAST OF THE 4 FOOT CHAIN LINK FENCE, AND 17.0 M (55.8 FT) SOUTHEAST OF A POLE WITH ELECTRIC METER.

USER NOTES - CBL USERS SHOULD TAKE CARE IN PLUMBING OVER ALL POINTS. ELEVATIONS ARE FOR CBL USE ONLY. FOR INTERVISIBILITY BETWEEN ALL BASE LINE POINTS, SET TRIPODS OVER THE MARKS AT APPROXIMATELY THE FOLLOWING HEIGHTS: 0 METER POINT AT $1.772 \mathrm{M}$ (5.814 FT), 122 METER POINT AT $1.208 \mathrm{M}$ (3.963 FT), 429 METER POINT AT 1.473 M (4.833 FT), 734 METER POINT AT 1.603 M (5.259 FT) AND THE 1044 METER POINT AT $1.885 \mathrm{M}(6.184 \mathrm{FT})$.

THIS BASE LINE WAS ESTABLISHED IN CONJUNCTION WITH THE NEW YORK STATE DEPARTMENT OF TRANSPORTATION AND A. JAMES DEBRUIN, CIVIL ENGINEER AND LAND SURVEYOR.

FOR MORE INFORMATION PLEASE CONTACT THE NEW YORK STATE DEPARTMENT OF TRANSPORTATION REGION 10 SURVEY OFFICE, HAUPPAUGE, NEW YORK AT (516) 952-6682.

IUS DEPARTMENT OF COMMERCE - NOAA NOS - NATIONAL GEODETIC SURVEY SILVER SPRING MD 20910 - MARCH 4, 1998

$$
\begin{array}{ll}
\text { CALIBRATION BASE LINE DATA } & \text { QUAD: N400734 } \\
\text { BASE LINE DESIGNATION: HEMPSTEAD CBL } & \text { NEW YORK } \\
\text { PROJECT ACCESSION NUMBER: } 15483 & \text { NASSAU COUNTY }
\end{array}
$$

\begin{tabular}{|c|c|c|c|c|c|c|}
\hline FROM STATION & ELEV.(M) TO STATION & ELEV.(M) & $\begin{array}{l}\text { ADJ. DIST.(M) } \\
\text { HORIZONTAL }\end{array}$ & $\begin{array}{l}\text { ADJ. DIST.(M) } \\
\text { MARK - MARK }\end{array}$ & $\begin{array}{l}\text { STD. } \\
\text { ERROR(MM) }\end{array}$ & EXPLANATION \\
\hline 0 NORTH BASE & 26.816122 P.O.L. 3 & 26.896 & 122.4987 & 122.4987 & 0.1 & \multirow{4}{*}{ Horizontal distance } \\
\hline 0 NORTH BASE & 26.816429 P.O.L. 2 & 25.500 & 429.8936 & 429.8956 & 0.1 & \\
\hline 0 NORTH BASE & 26.816734 P.O.L. 1 & 24.795 & 734.7888 & 734.7916 & 0.1 & \\
\hline 0 NORTH BASE & 26.8161044 SOUTH BASE & 23.732 & 1044.5262 & 1044.5307 & 0.2 & \\
\hline 122 P.O.L. 3 & 26.896429 P.O.L. 2 & 25.500 & 307.3950 & 307.3981 & 0.1 & \multirow{6}{*}{ Horizontal spatial chord distance } \\
\hline 122 P.O.L. 3 & 26.896734 P.O.L. 1 & 24.795 & 612.2902 & 612.2938 & 0.1 & \\
\hline 122 P.O.L. 3 & 26.8961044 SOUTH BASE & 23.732 & 922.0276 & 922.0330 & 0.1 & \\
\hline 429 P.O.L. 2 & 25.500 734 P.O.L. 1 & 24.795 & 304.8952 & 304.8960 & 0.1 & \\
\hline 429 P.O.L. 2 & 25.5001044 SOUTH BASE & 23.732 & 614.6326 & 614.6351 & 0.1 & \\
\hline 734 P.O.L. 1 & 24.7951044 SOUTH BASE & 23.732 & 309.7374 & 309.7392 & 0.1 & \\
\hline
\end{tabular}

NEAREST TOWN: HEMPSTEAD

LIST OF ADJUSTED DISTANCES (FEBRUARY 11, 1998)

Figure 17. Example of a National Geodetic Survey baseline calibration sheet for Hempstead, New York. Derived horizontal distances are used to test the electronic distance measurement uncertainty. Datasheet can be accessed at https://geodesy.noaa.gov/CBLINES/ index.shtml. 


\section{Sources of Measurement Uncertainty for Trigonometric Leveling}

Previous sections in this manual have identified and described sources of uncertainty that are caused by the surveyor and the trigonometric-leveling survey equipment. The purpose of this section is to describe the implications of constant and distance-weighted error sources on the VD and the mark-to-mark VD of a trigonometric-leveling measurement (fig. 8). The VD is height difference between the horizontal plane of the instrument and the horizontal plane of the prism and is determined by multiplying the measured slope distance by the cosine of the zenith angle. A positive VD indicates the target (prism) is above the horizontal plane of the instrument, and a negative VD indicates it is below. The mark-to-mark VD is defined as the height difference between the survey marker at the station and the survey marker at the objective point. It is determined from the four fundamental field measurements: (1) the instrument height (HI) of the total station above the survey marker, (2) the slope distance (SD) from the total station to the reflector at the objective point, (3) the zenith angle (ZA) from the total station to the reflector, and (4) the sight height (SH) of the reflector above the survey marker at the objective point (fig. 8). The mark-to-mark VD can be calculated by adding the VD (positive or negative) to the $\mathrm{HI}$ and then subtracting the SH (fig. 8) using the following equation:

$$
V D_{\text {mark-to-mark }}=H I+V D-S H
$$

where

$$
\begin{array}{cl}
V D_{\text {mark-to-mark }} & \text { is the mark-to-mark VD, in feet; } \\
V D & \text { is the VD, in feet; } \\
H I & \text { is the height of the total station, in feet; } \\
S H & \text { is the height of the prism, in feet; }
\end{array}
$$

The elevation of the survey marker at the objective point is then determined by adding the mark-to-mark VD (positive or negative) to the known elevation of the trusted survey marker at the station (fig. 8). Sources of error that can affect the four components of the mark-to-mark VD (HI, SH, ZA, and SD) are described in the following section.

\section{Electronic Distance Measurement}

An EDMI measures the slope distance between the instrument and a reflector by transmitting a pulsed or modulated electromagnetic signal that is typically infrared or visible light. The returned signal is then processed by the EDMI and a slope distance is determined using a phase difference, or two-way travel time method, which is described in the "Trigonometric-Leveling Equipment" section. The greatest source of uncertainty for an EDMI is random error that is associated with the manufacturer's inaccurate determination of the velocity of light during the measurement, modulation frequency, and refractive index (Ogundare, 2016). These sources of uncertainty are typically specified by the manufacturer as two error components - constant and distance-weighted; constant error is fixed and is the same for every EDM, and distance-weighted error, or scale error, is proportional to the distance of the EDM and increases as measurement distance increases (table 2). The manufacturer-specified uncertainty of the EDMI should be verified in the field using calibrated baselines and the procedure described in the "Preparing for Trigonometric Leveling" section.

The uncertainty of the slope distance measurement (EDM) caused by the fixed and scale errors of the EDMI has implications on the uncertainty of the VD. The VD, which is defined as the height difference between the horizontal plane of the total station and the horizontal plane of the reflector, is proportional to both the slope distance and the cosine of the zenith angle (fig. 8). For example, an EDMI with a manufacturer-specified fixed and distance-weighted uncertainty of $2 \mathrm{~mm}$ plus $2 \mathrm{ppm}$ could potentially result in a VD error of $\pm 0.0012 \mathrm{ft}$ over a measurement distance of $100 \mathrm{ft}$ and a zenith angle of $80^{\circ}$; over a $500 \mathrm{ft}$ measurement distance and a zenith angle of $60^{\circ}$, the potential VD error could increase to $0.0038 \mathrm{ft}$ (fig. 18; table 2). The potential VD uncertainty approaches the maximum potential EDMI uncertainty (fixed and scale) for steep sightings (zenith angle approaches $0^{\circ}$ and $180^{\circ}$ ). Using the previous example for an EDMI with an uncertainty of $2 \mathrm{~mm}$ plus 2 ppm and a measurement distance of $500 \mathrm{ft}$, the maximum potential VD uncertainty error would be about $\pm 0.0076 \mathrm{ft}(2.31 \mathrm{~mm})$ for a zenith angle of $0^{\circ}$ (fig. 18). Although it is quite rare to measure a zenith angle near $0^{\circ}$ or $180^{\circ}$ during a trigonometric-leveling campaign, it is important to understand the effect of a small EDM error on VD uncertainty and the trigonometric relation between angles and distances. Furthermore, one of the major benefits of running trigonometric levels is that less instrument setups may be required in project areas that have variable topography (as compared to differential levels), so the surveyor must pay attention to the measured zenith angle for the purpose of minimizing VD uncertainty related to the slope distance measurement. Other major sources of EDM uncertainty are from centering errors of the total station and the reflector (prism) and from error associated with atmospheric observations, which are described in the "Testing the Electronic Distance Measurement" section.

\section{Angular Precision of the Total Station}

Manufacturers of total stations determine the angular precision according to industry recognized standards from organizations such as the International Organization for Standardization and the German Institute for Standards (Trimble, Inc., 2017; Leica Geosystems, 2017c). These organizations specify field and laboratory procedures to determine the precision (repeatability) of horizontal and zenith angles measured with a total station. Angular precision (sometimes called angular accuracy by the manufacturer) is typically 
Table 2. Sources of measurement uncertainty for a trigonometric-leveling survey.

[VD, vertical difference; \pm , plus or minus; EDM, electronic distance measurement; EDMI, electronic distance measurement instrument; F1, direct instrument orientation; F2, reverse instrument orientation; - , negligible; ND, no data]

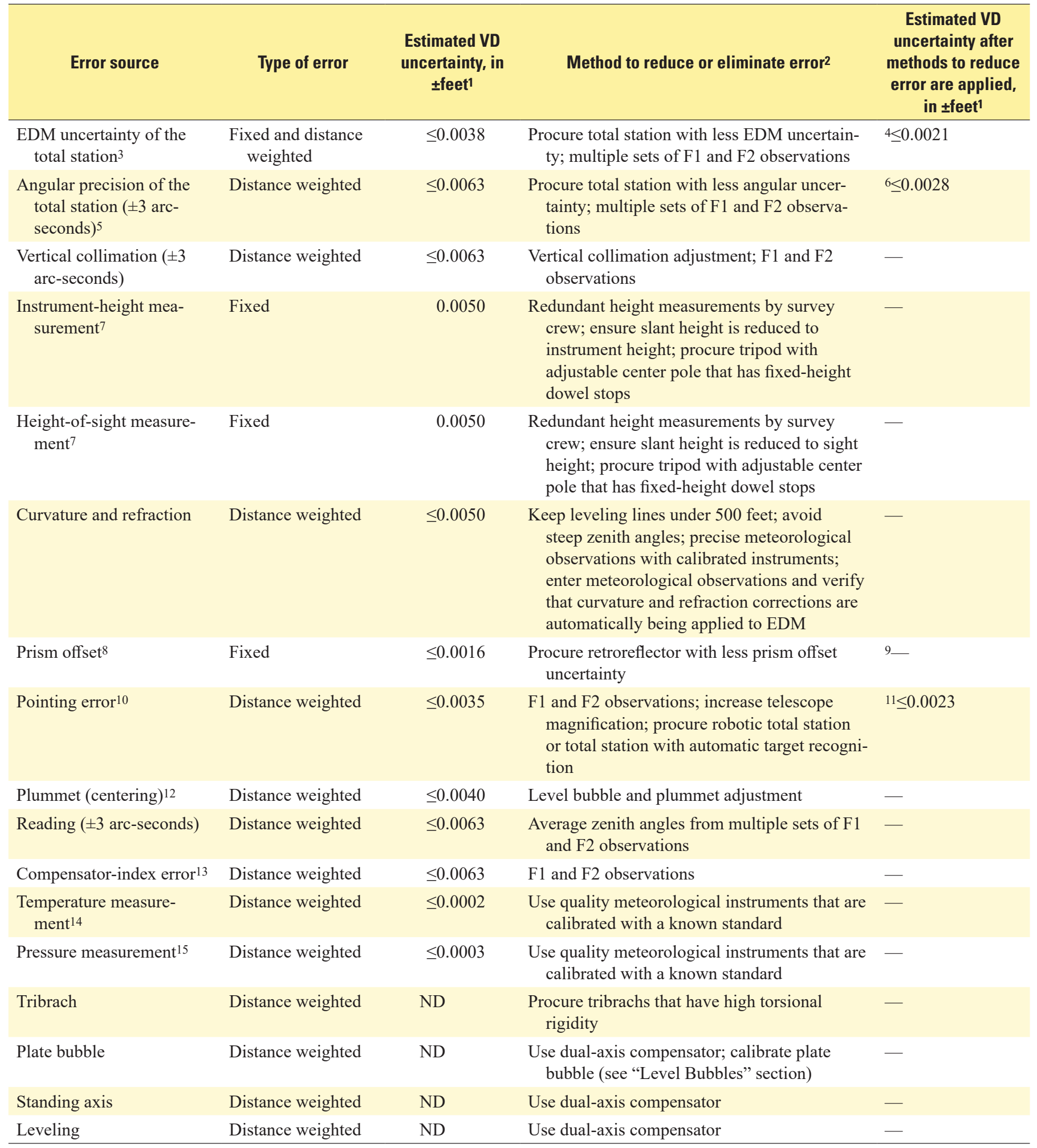




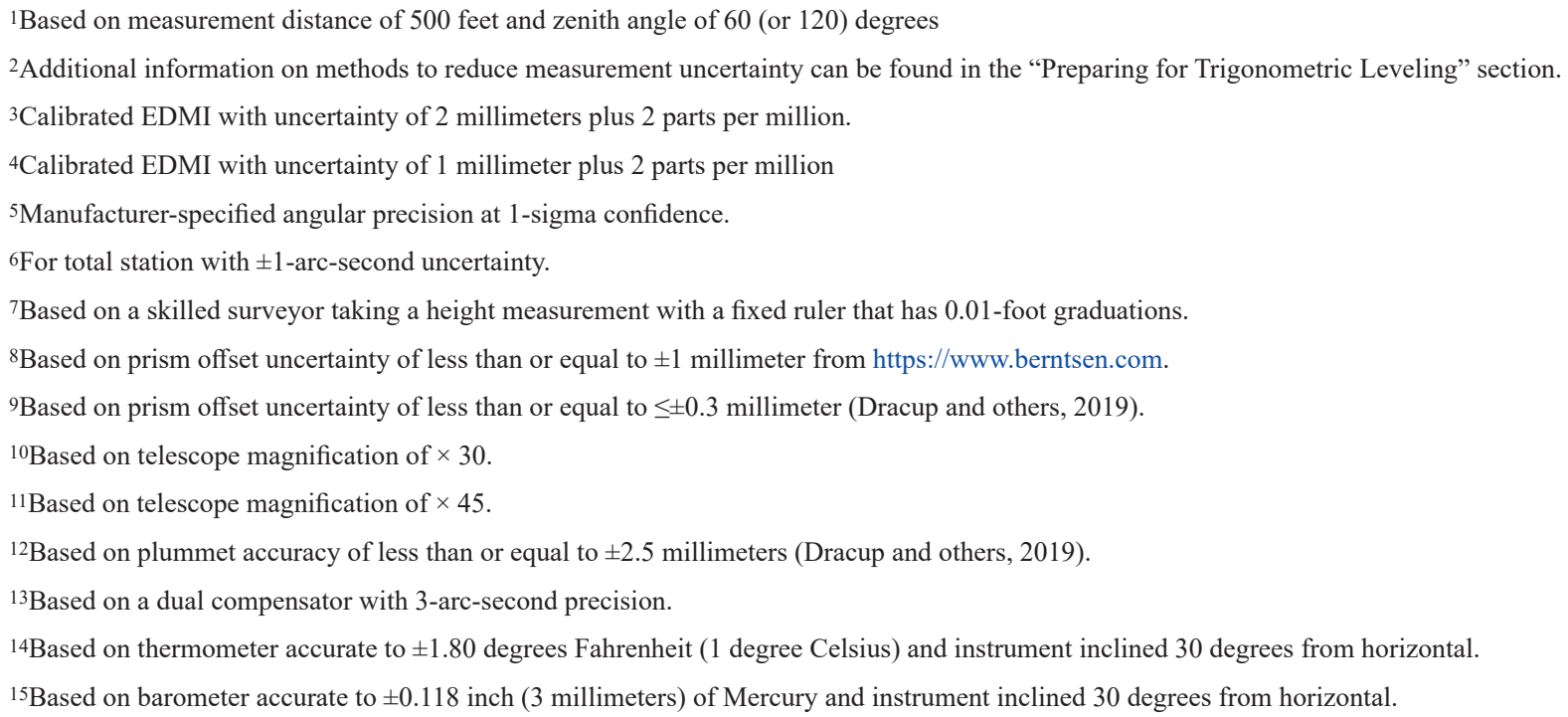

expressed by the manufacturer in arc-seconds at 1-sigma confidence (about 68 percent) on instrument specification or data sheets. This is an important concept because often the manufacturer-specified angular precision of the instrument is the standard deviation of the mean direction from multiple F1 and $\mathrm{F} 2$ readings and is not the angular precision of a single $\mathrm{F} 1$ observation. For example, a total station with a manufacturerspecified 5" angular precision has a 68-percent chance of repeating the mean of previous $\mathrm{F} 1$ and F2 observations to a single target (that is, not an angle) within 5". For this reason, it is important to understand the industry standards that are used by manufacturers to determine the angular precision of the total station, which is sometimes indicated as a superscript next to the angular precision specification of the total station on the manufacturer's instrument data sheet. Angular precision derived from the mean of F1 and F2 observations may mislead many who assume the derivation is representative of only one singular F1 measurement. As derived, it may be implied that a total station purporting an angular precision of $5 "$ on average underperforms that level of precision.

If the manufacturer-specified angular precision is applied to a horizontal angle measurement, the actual precision of the angle at 1 sigma or 68 -percent confidence will be 1.4 times the manufacturer's specification if the instrument is pointed at two targets (the backsight and the foresight) and measurements are made in F1 and F2 - both of which are standard operating procedures during a trigonometric-leveling survey. The uncertainty can be calculated by

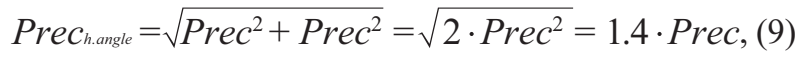

where

Prec $_{\text {h.angle }}$

is the measured angle precision measured in two faces and

Prec is the manufacturer's stated precision for a direction measured in two faces.
However, zenith angles are measured with a single pointing because they are theoretically referenced to the upward vertical direction (the opposite of the direction of gravity). Therefore, the manufacturer's stated precision can be used directly as the uncertainty at the 68-percent confidence level in a zenith angle observation if the observation is made in F1 and F2 (Joseph Paiva, GeoLearn, LLC, written commun., 2019).

The VD uncertainty caused by the angular precision of the total station is proportional to the measurement distance and the cosine of the zenith angle (fig. 8; tables 1 and 3). For example, a 5 " total station may cause a VD uncertainty of $\pm 0.0048 \mathrm{ft}$ for a slope distance of $200 \mathrm{ft}$ along a horizontal line of sight; however, that potential error increases by a factor of five $( \pm 0.024 \mathrm{ft})$ for a slope distance of $1,000 \mathrm{ft}$ because the angular precision uncertainty of the total station is a distanceweighted error. It is recommended that measured slope distances are less than $500 \mathrm{ft}$ to reduce VD uncertainty because of the angular uncertainty of the total station. Some modern total stations are capable of measuring angles of 1 " or less, which can substantially reduce VD error but may be cost prohibitive because the price of a total station is usually proportional to the angular precision (table 1). Furthermore, because of the limitations of the operator and resolution of the telescope, it is uncommon for instruments rated at 1 " or less to reach that precision with just a single set of F1 and F2 observations. Observation and experience indicate that three or four sets may be needed, where the random error created by the abovementioned sources can be averaged out; conversely, multiple $\mathrm{F} 1$ and $\mathrm{F} 2$ observations can also improve the angular precision of a construction-grade total station $\left(>5^{\prime \prime}\right)$ because systematic error can be averaged out.

Measurement uncertainty because of the angular precision of the total station and the constant and distantweighted error associated with the EDMI can persist during a trigonometric-leveling survey because these sources of error may be reduced, but not eliminated, using traditional quality assurances. Table 3 lists the maximum potential VD 
A
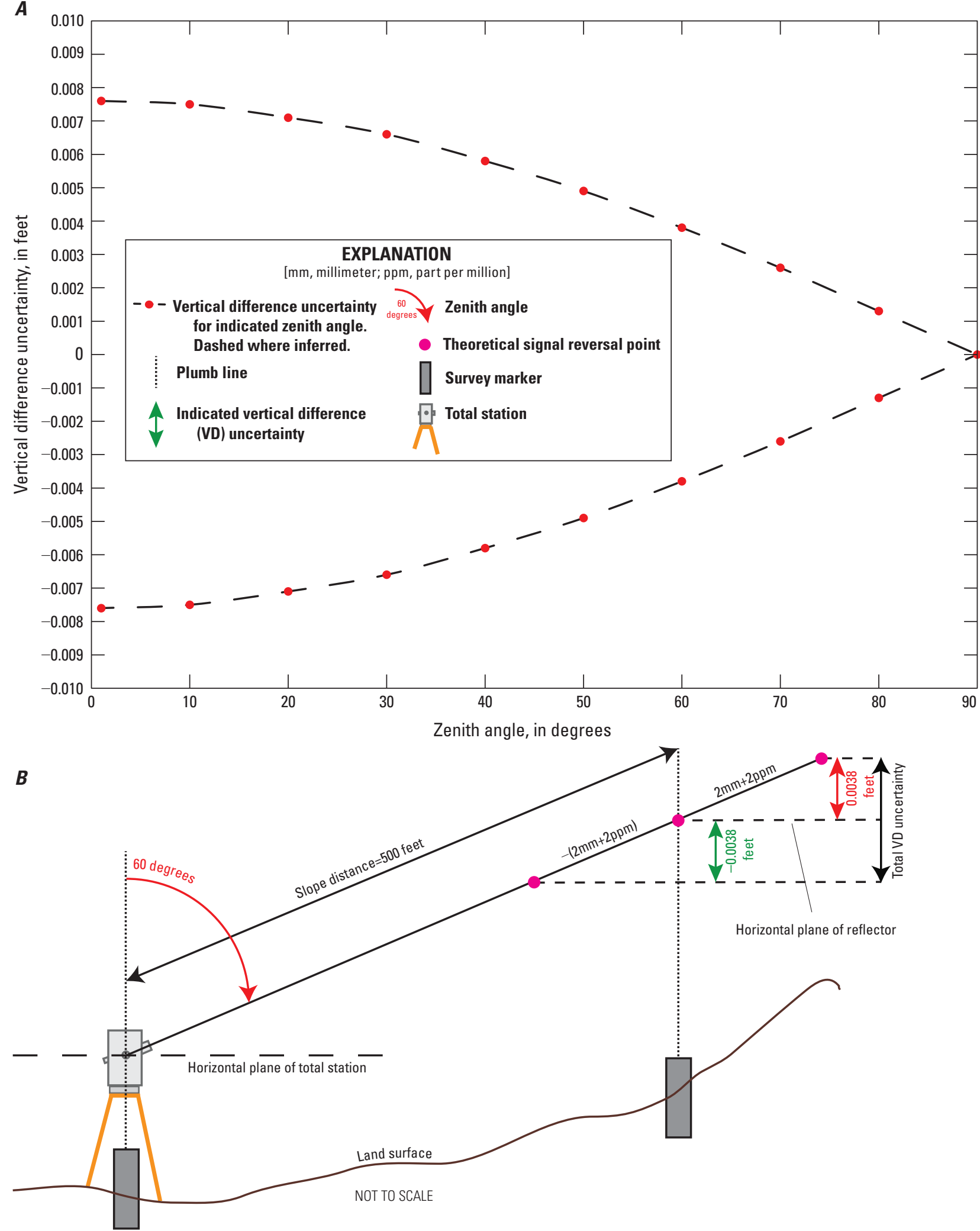

Figure 18. Implications of electronic distance measurement uncertainty on the vertical difference. $A$, Uncertainty of vertical difference as a function of the observed zenith angle for a total station with a 2-millimeter fixed error and 2 parts per million distance-weighted error for a slope distance of 500 feet; and $B$, The effect of a 2 millimeter plus 2 parts per million uncertainty on the vertical difference for a slope distance of 500 feet and a zenith angle of 60 degrees. 
Table 3. Estimated maximum potential vertical difference uncertainty for a total station with 3 arc-second angular precision and an electronic distance measurement instrument uncertainty of 2 millimeters plus 2 parts per million.

[ \pm , plus or minus]

\begin{tabular}{cccccccccccc}
\hline $\begin{array}{c}\text { Zenith } \\
\text { angle, } \\
\text { in degrees }\end{array}$ & \multicolumn{9}{c}{ Vertical difference uncertainty, in \pm feet, at each measurement distance, in feet } & \multicolumn{2}{c}{$\begin{array}{c}\text { Zenith angle, } \\
\text { in degrees }\end{array}$} \\
\hline 89 & $\mathbf{1 0 0}$ & $\mathbf{2 0 0}$ & $\mathbf{3 0 0}$ & $\mathbf{4 0 0}$ & $\mathbf{5 0 0}$ & $\mathbf{6 0 0}$ & $\mathbf{7 0 0}$ & $\mathbf{8 0 0}$ & $\mathbf{9 0 0}$ & $\mathbf{1 , 0 0 0}$ \\
8.002 & 0.003 & 0.004 & 0.006 & 0.007 & 0.009 & 0.010 & 0.012 & 0.013 & 0.015 & 91 \\
88 & 0.002 & 0.003 & 0.005 & 0.006 & 0.008 & 0.009 & 0.010 & 0.012 & 0.013 & 0.015 & 92 \\
86 & 0.002 & 0.003 & 0.005 & 0.006 & 0.008 & 0.009 & 0.011 & 0.012 & 0.014 & 0.015 & 93 \\
85 & 0.002 & 0.003 & 0.005 & 0.006 & 0.008 & 0.009 & 0.011 & 0.012 & 0.014 & 0.015 & 94 \\
84 & 0.002 & 0.004 & 0.005 & 0.006 & 0.008 & 0.009 & 0.011 & 0.012 & 0.014 & 0.015 & 95 \\
83 & 0.002 & 0.004 & 0.005 & 0.007 & 0.008 & 0.009 & 0.011 & 0.012 & 0.014 & 0.015 & 96 \\
82 & 0.002 & 0.004 & 0.005 & 0.007 & 0.008 & 0.010 & 0.011 & 0.013 & 0.014 & 0.015 & 97 \\
81 & 0.002 & 0.004 & 0.005 & 0.007 & 0.008 & 0.010 & 0.011 & 0.013 & 0.014 & 0.016 & 98 \\
80 & 0.002 & 0.004 & 0.005 & 0.007 & 0.008 & 0.010 & 0.011 & 0.013 & 0.014 & 0.016 & 99 \\
79 & 0.003 & 0.004 & 0.006 & 0.007 & 0.008 & 0.010 & 0.011 & 0.013 & 0.014 & 0.016 & 100 \\
78 & 0.003 & 0.004 & 0.006 & 0.007 & 0.009 & 0.010 & 0.012 & 0.013 & 0.014 & 0.016 & 101 \\
77 & 0.003 & 0.004 & 0.006 & 0.007 & 0.009 & 0.010 & 0.012 & 0.013 & 0.015 & 0.016 & 102 \\
76 & 0.003 & 0.004 & 0.006 & 0.007 & 0.009 & 0.010 & 0.012 & 0.013 & 0.015 & 0.016 & 103 \\
75 & 0.003 & 0.005 & 0.006 & 0.007 & 0.009 & 0.010 & 0.012 & 0.013 & 0.015 & 0.016 & 104 \\
\hline
\end{tabular}

uncertainty for various measurement distances and zenith angles for a total station with an angular uncertainty of $3^{\prime \prime}$ equipped with an EDMI of $2 \mathrm{~mm}(0.007 \mathrm{ft})$ constant error plus $2 \mathrm{ppm}$ scale error. The VD uncertainty values listed in table 3 are the combined estimates of the two sources of error.

\section{Vertical Collimation}

Vertical collimation error is a defect that causes a misalignment between the true and observed (apparent) line of sight along the vertical plane of the instrument, which can be measured on the vertical circle (figs. 7 and $9 B$; table 2; Ogundare, 2016). Methods for determining and correcting for vertical collimation error are described in the "Preparing for Trigonometric Leveling" section. In summary, taking zenith angle measurements in F1 and F2 can reduce vertical collimation error because the uncertainty in the $\mathrm{F} 1$ cancels out the uncertainty in the F2 because the error is equal but in the opposite direction. Once determined, the vertical collimation error can be stored so the adjustment is automatically applied to subsequent vertical circle readings (zenith angles; Leica Geosystems, 2013). Vertical collimation error propagates similarly to angular precision uncertainty and has the same effect on the VD because it is a distance-weighted error that is proportional to the measured slope distance and the cosine of the zenith angle.

\section{Measured Heights of Surveying Instruments}

USACE (2007) reports that combined error from $\mathrm{HI}$ and SH measurements can range from 0.005 to $0.1 \mathrm{ft}$ per setup (table 2). The uncertainty resulting from these height measurements is constant and is not a function of measurement distance. Historically, instrument heights were determined with a fixed ruler by measuring the distance from the top of the survey marker to the tilting axis of the total station that is usually indicated by a notch, mark, or groove in the side of the instrument (fig. 3). The uncertainty is introduced when the surveyor assumes this measurement is the HI, when in fact it is a slope distance, or slant height, that is slightly longer than the true HI. The HI can be calculated from the slant height if the angle $(\beta)$ between the slant height and the plumb line (vertical axis) can be determined, or if the orthogonal distance $(R)$ from the notch (mark or groove) on the side of the total station to the vertical axis of the instrument is known (fig. 19A). This method also requires that the slant height measurement is a straight line from the survey marker to the notch on the side of the total station. Some total stations have a notch below the tilting axis of the instrument, which is used as a measuring point to obtain a highly accurate HI. The slant height measurement is made from the survey marker to a notch below the tilting axis; and the data-collection device (integrated or external) automatically reduces the slant height to the vertical equivalent and then adds the known offset between measuring point notch and the tilting axis to obtain the HI (fig. 19B). 
$\boldsymbol{A}$

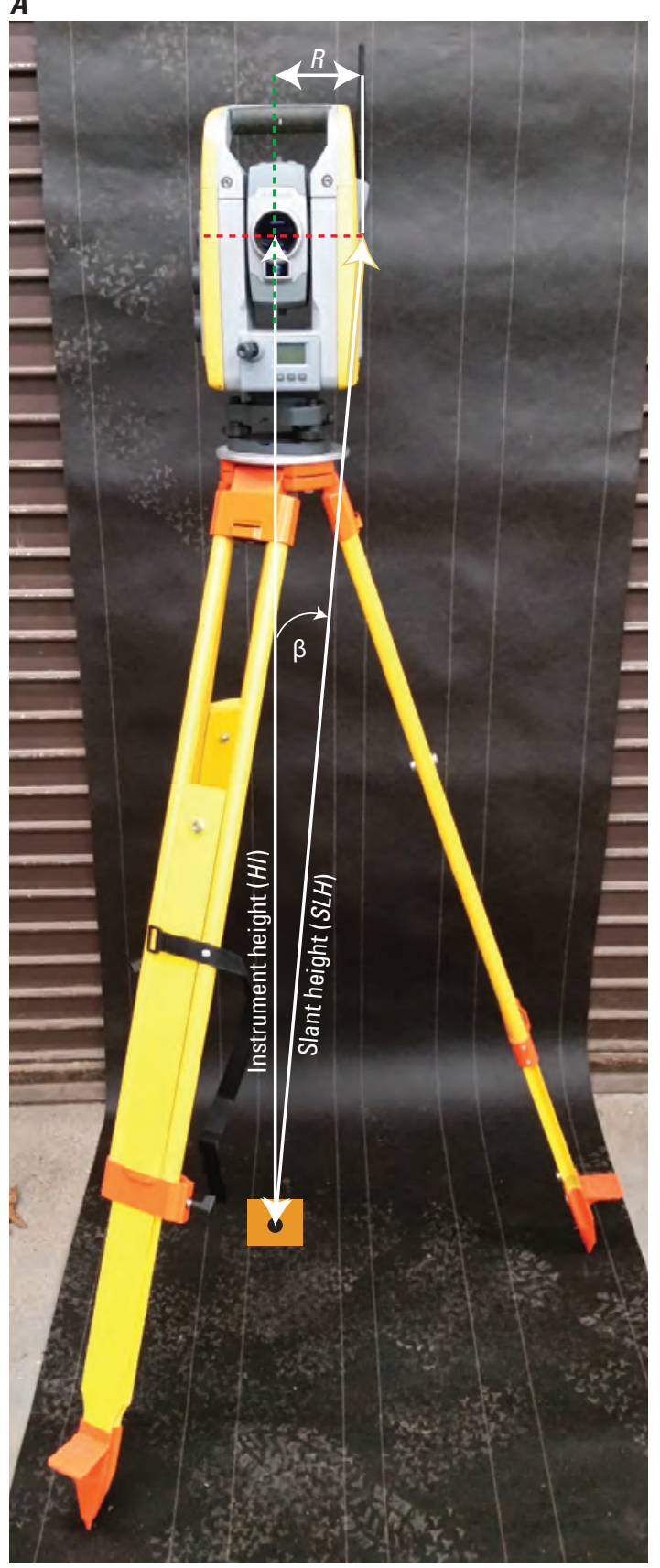

$H I=\cos (\beta) \times(S L H)$

$H I=\sqrt{(S L H)^{2}-(R)^{2}}$
$B$

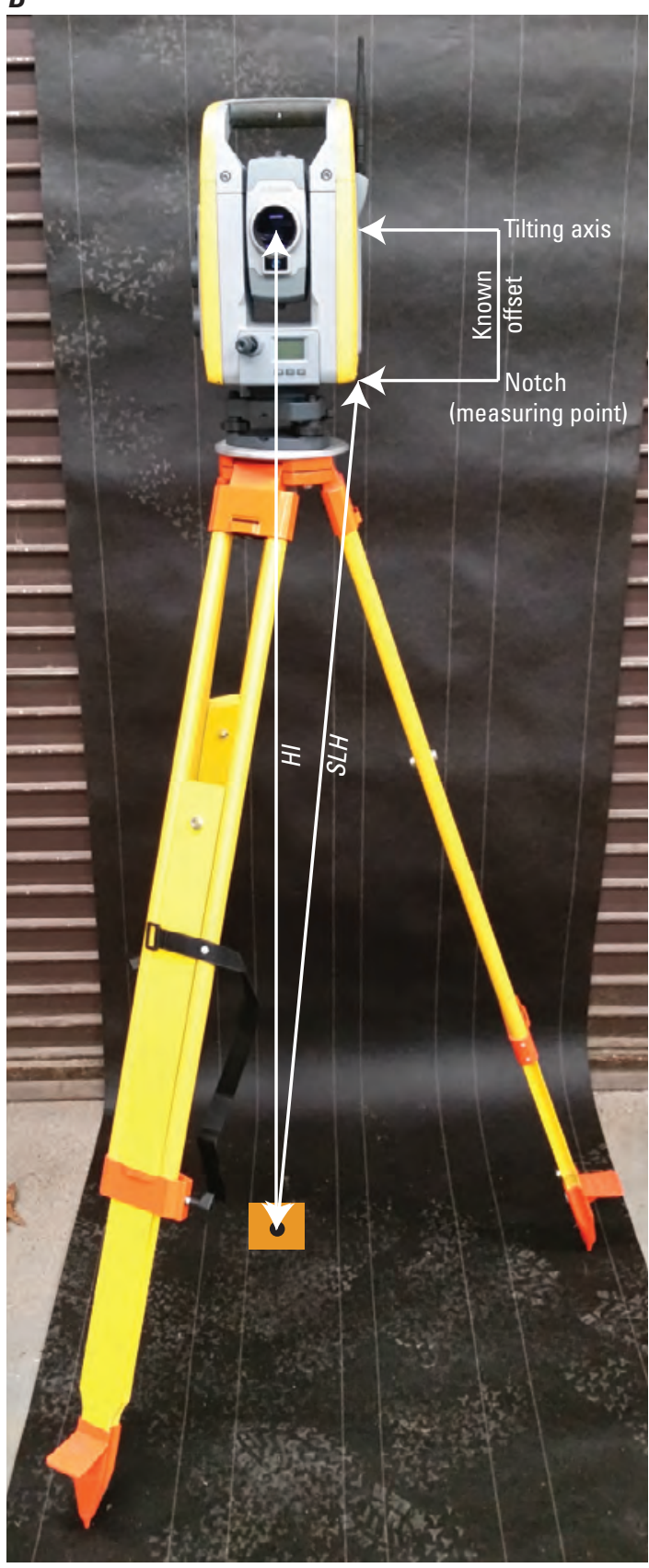

$H I=S L H$ (reduced to vertical equivalent) + known offset

\section{EXPLANATION}

- Survey marker

Vertical axis (standing axis)

. . . . . . Horizontal axis (tilting axis)

Figure 19. Relation between the slant height and the instrument height. $A$, operator must use trigonometry to calculate the instrument height; and $B$, operator measures the slant height to a measuring point and then the instrument height is automatically calculated by the total station. 
Some data-collection devices automatically calculate the $\mathrm{HI}$ based on the measured slant height because $R$ and $\beta$ are both known (fig. 19A); however, the instrument-derived HI should be verified with a manual calculation before starting a trigonometric-leveling survey. In some cases, the integrated microprocessor must be programmed to automatically calculate the HI. If a paired, external data-collection device is being used to automatically calculate the HI based on the slant height, ensure the correct total station make and model are selected because $R$ and $\beta$ vary among surveying instruments even if in the same series or model number from the same manufacturer. For modern total stations, it is likely that the integrated data-collection device automatically reduces the measured slant height to an HI; the user manual should be consulted to learn more about this functionality.

Similar to HIs, height measurements of prisms mounted to conventional tripods, bipods, or operator-leveled rods can also introduce uncertainty. A prism-height measurement, or sight height ( $\mathrm{SH}$ ), is considered the distance measured along a plumb line from the top of the survey marker to the center of the prism. The center of the prism is the apex formed by the prism facets and is usually indicated by a notch or groove on the side of the prism housing or target plate (fig. 2). There is less potential measurement uncertainty for slant-height measurements for prisms than for total stations because the distance from the side of the total station to the center of the total station $(R)$, is greater than the distance from the side of the prism housing to the center (apex) of the prism $(\cos [\beta] \approx 1)$. However, the measurement is still considered a slant height because it is impossible to measure a plumb distance from the top of the survey marker to true center of the prism. Furthermore, uncertainty can still be introduced by operator blunders or from transcription errors.

Tripods with an adjustable center pole that has dowel stops (typically at 1.5, 1.8, and $2.0 \mathrm{~m}$ [4.921, 5.906, and $6.562 \mathrm{ft}$, respectively]) may be a compelling alternative as the mounting platform for the total station and prisms. They reduce or eliminate uncertainty that is introduced by the operator from blunders such as reading the fixed ruler incorrectly and interpolating between height increments; by improper determination of $\beta$ and, therefore, improper determination of the reduced slant height; and from transcription errors, such as entering an erroneous height into the data-collection device. An additional benefit of a tripod with a center pole is that centering errors are also reduced or eliminated if the center pole is plumb and the level bubble has been properly adjusted, which is described in the "Preparing for Trigonometric Leveling" section. To properly account for the $\mathrm{HI}$ in the data-collection device, the operator must add the offset, or the distance from the top of the tripod platform (or bottom of the tribrach) to the tilting (trunnion) axis of the instrument, to the height of the tripod (usually $1.5,1.8$, or $2.0 \mathrm{~m}$ ). Like the total station, the distance from the tripod platform (or bottom of the tribrach) to the center of the prism must be added to the tripod height to determine the SH. These offset distances are constant, are typically a round number, and may be indicated on the equipment specification sheets. Components of a traverse kit should be from the same manufacturer to ensure offset distances. In any case, offset distances should be verified by the operator with a measurement.

Uncertainty from HIs for traditional adjustable tripods can be substantially reduced using the previously described techniques that require a fixed ruler measurement of the slant height and an estimate of the angle ( $\beta$ ); a fixed ruler measurement of the slant height and the offset between the side of the instrument and center $(R)$; or a slant height measurement to a measuring point of known offset to the tilting axis. These simple trigonometric relations, combined with the ability of a skilled surveyor to make an accurate height measurement with a fixed-height ruler and careful accounting of the measurements, can substantially reduce the $\mathrm{HI}$ and $\mathrm{SH}$ measurement uncertainty. Furthermore, redundant height measurements should be made by the survey crew at the backsight, station, and foresight to ensure quality observations and should be within $|\leq 0.005| \mathrm{ft}$.

\section{Curvature and Refraction}

In many literature sources, measurement uncertainty resulting from Earth's curvature $(c)$ and atmospheric refraction $(r)$ are combined into a single factor $(h)$ that estimates the effect of these systematic error sources on the VD (fig. 20; table 2; Brinker and Minnick, 1995; USACE, 2007; Ogundare, 2016). Earth's surface can be modeled as an ellipse with a radius of $20.9 \times 10^{6} \mathrm{ft}$ for coarse estimates; for more precise estimates, Earth's radius depends on the location of the observer on Earth's surface (Brinker and Minnick, 1995). The correction for curvature (c) for a modeled ellipse is about $0.667 \mathrm{ft}$ multiplied by the square of the measurement distance in miles. For example, a surveyor could expect an error of approximately $0.006 \mathrm{ft}$ because of Earth's curvature for a $500 \mathrm{ft}$ measurement distance with a total station: $c=(500 \mathrm{ft} \div 5,2$ $80 \mathrm{ft})^{2} \times 0.667 \mathrm{ft}=0.006 \mathrm{ft}$.

This hypothetical error would be subtracted from the derived VD to obtain a VD that is adjusted for the effect of Earth's curvature (figs. 8 and 20). However, because of atmospheric conditions (more specifically, air density variations along the path of the EDM signal), another correction must be considered that is related to the refraction of light.

Atmospheric conditions, primarily varying densities of air between the total station and the prism (foresight or backsight), can modify the direction and velocity of a transmitted signal (light beam) as it propagates along an optical path (Brinker and Minnick, 1995; Ogundare, 2016). Variations in atmospheric density cause the line of sight to refract or bend in the direction of increasing air density (Schomaker and Berry, 1981). These variations seem to be primarily a function of air temperature. In some cases, a transmitted EDM signal can be bent (curved) away from the ideal path along the horizontal plane of the instrument and towards Earth's surface (fig. 20). As atmospheric conditions change, the velocity and 


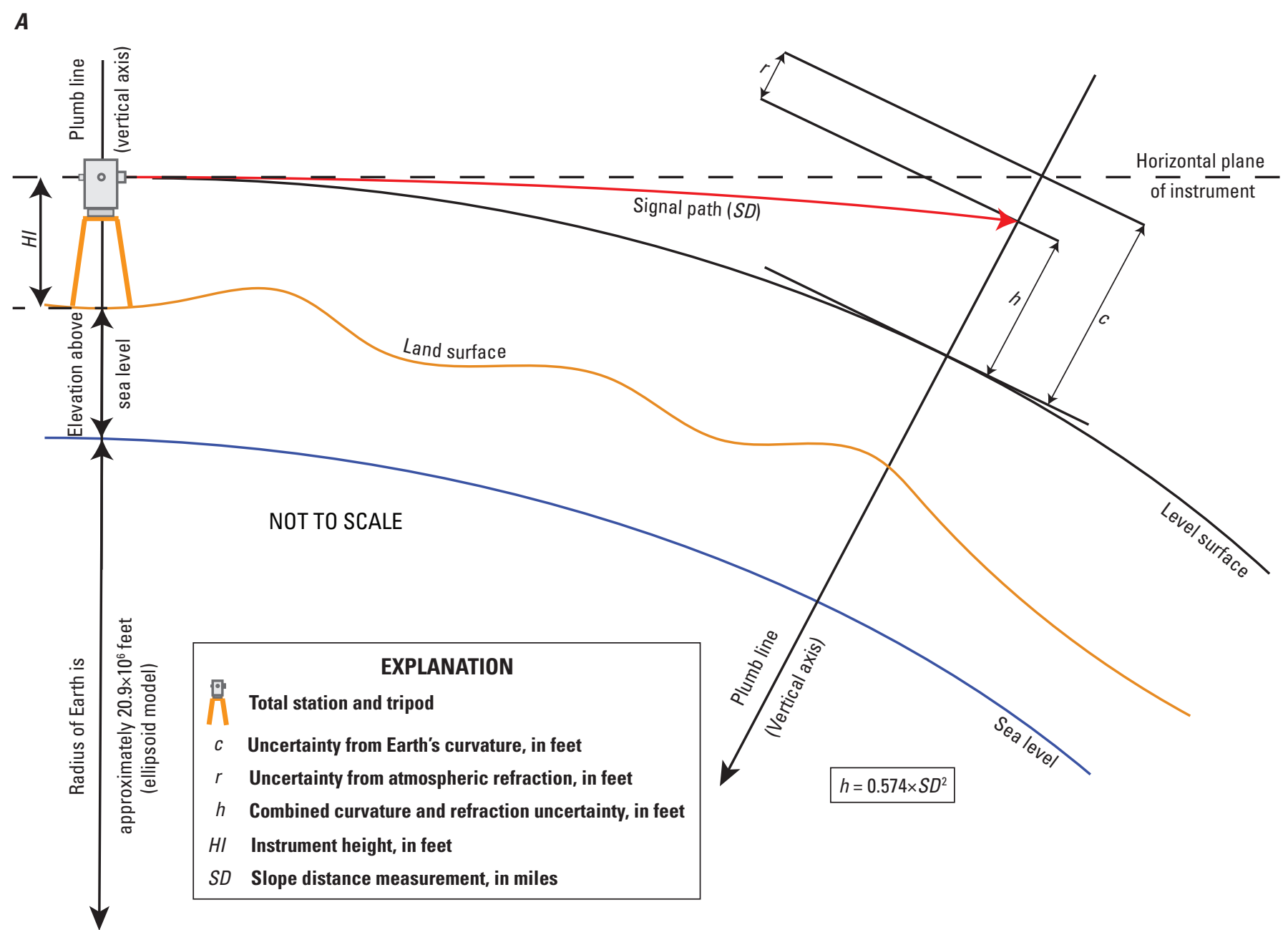

Figure 20. Combined measurement uncertainty for Earth's curvature and atmospheric refraction, and a data-collection device screen. $A$, combined measurement uncertainty $(h)$ due to Earth's curvature $(c)$ and atmospheric refraction $(r)$. Sea level is approximately parallel to the level surface of the instrument. Plumb lines orthogonally intersect sea level and the level surface of the instrument (modified from Brinker and Minnick, 1995); and B, photograph of screen from data-collection device showing the field to enter barometric pressure and ambient air temperature so corrections for $c$ and $r$ are automatically applied to subsequent measurements. 


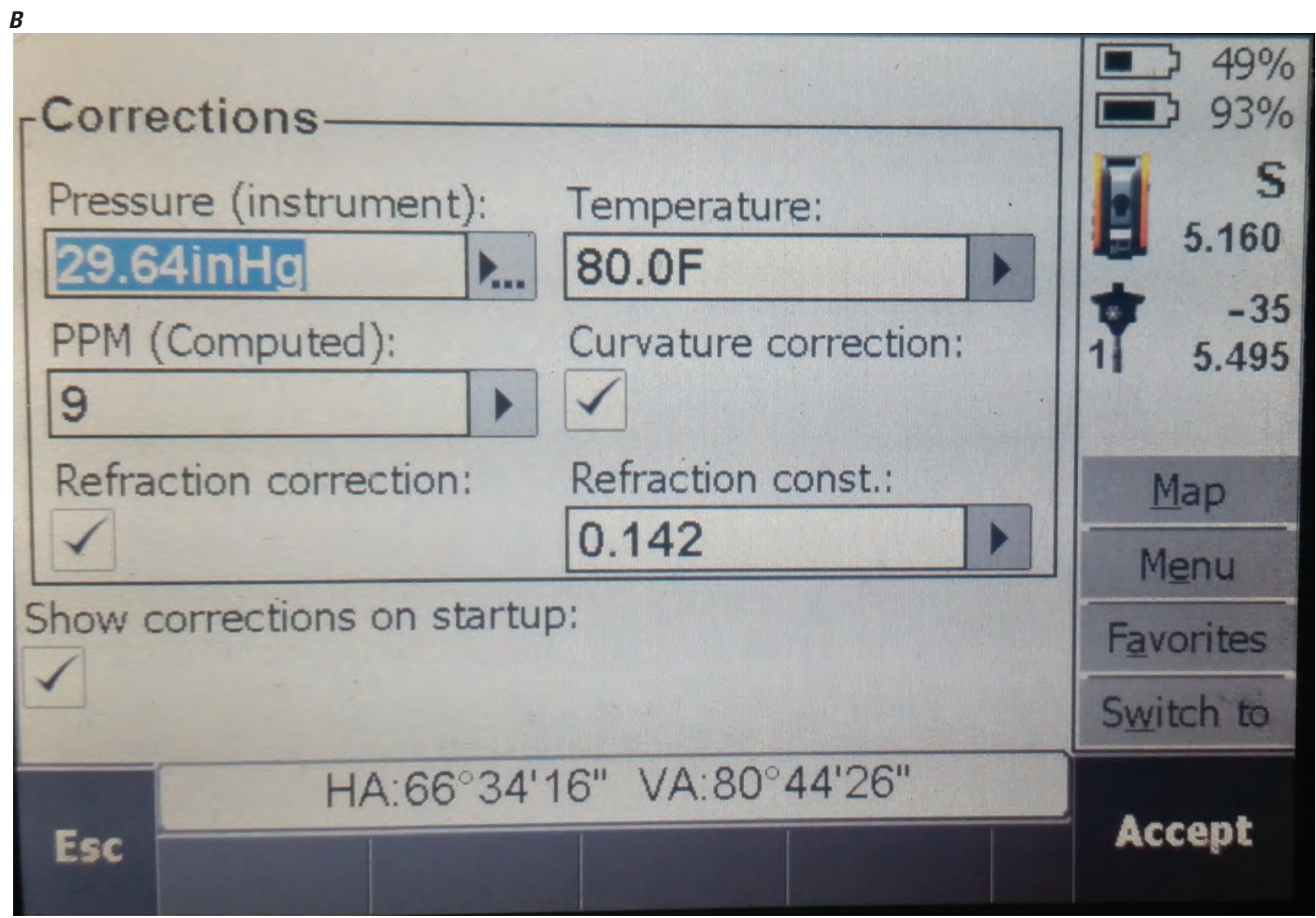

Figure 20. Combined measurement uncertainty for Earth's curvature and atmospheric refraction, and a data-collection device screen. $A$, combined measurement uncertainty $(h)$ due to Earth's curvature $(c)$ and atmospheric refraction $(r)$. Sea level is approximately parallel to the level surface of the instrument. Plumb lines orthogonally intersect sea level and the level surface of the instrument (modified from Brinker and Minnick, 1995); and $B$, photograph of screen from data-collection device showing the field to enter barometric pressure and ambient air temperature so corrections for $c$ and $r$ are automatically applied to subsequent measurements. 
direction of the transmitted signal, and the resulting distance measurement, are consequently changed (Ogundare, 2016). This source of measurement uncertainty is called atmospheric refraction and can be estimated by making precise air temperature, barometric pressure, and relative humidity measurements along the measurement path. These meteorological measurements can be entered into the total station so that a correction for atmospheric refraction can be determined and automatically applied to the measurement (fig. 20B). Some total stations are equipped with integrated meteorological instruments, which allow the total station to automatically calculate corrections for atmospheric refraction. Varying densities of air can also distort the path of natural radiation (white light) that travels from the target or retroreflector to the instrument operator's eye, which can result in pointing error.

The correction for the effect of curvature and refraction are usually in opposite directions because refraction typically bends the EDM signal towards the Earth, whereas Earth's curved surface departs away from the line of sight of the EDMI (or total station) (fig. 20A; table 4). To simplify and relate these two sources of measurement uncertainty, the correction for atmospheric refraction is about $1 / 7(0.143)$ the correction for Earth's curvature. The correction for curvature and refraction are squarely proportional to the measurement distance and can be expressed using the following equation modified from Brinker and Minnick (1995):

$$
h=0.574 \times S D^{2},
$$

where

$h$ is the combined VD correction for curvature and refraction, in feet; and

$S D \quad$ is the slope distance measurement, in miles.

Simultaneous reciprocal leveling is a surveying technique that can be used to reduce observation error from curvature and refraction over longer measurement distances (Brinker and Minnick, 1995). It requires a total station at the station and foresight locations so that leveling observations can be made at the same time. Theoretically, concurrent observations made in opposite directions will cancel out measurement uncertainty caused by curvature and refraction; however, this method may be cost-prohibitive because it requires two total stations and two survey crews. Furthermore, refraction conditions along a leveling line are variable and direction dependent, so EDMs made in opposite directions will not necessarily travel along identical flight paths, so averaging reciprocal observations only distributes the error but does not necessarily eliminate it (Brinker and Minnick, 1995; Ogundare, 2016).

Refraction conditions are variable as a function of the horizontal distance from the total station, and as a function of height above the total station but are rarely equivalent. The effect of refraction in the vertical direction has been reported as one order of magnitude greater than the effect in the horizontal direction because vertical density gradients are substantially greater than horizontal density gradients (Vaniceck and Krakiwsky, 1986). The rate of change of vertical density gradients is also greater near the ground and decreases as the height above the ground increases (Schomaker and Berry, 1981; Kozlowski, 1998). In project areas with highly variable topography, substantial air temperature differences (density gradients) may be detected between the station and foresight locations. However, because these layers of air are usually parallel to land surface topography, trigonometric leveling is an ideal approach because line of sight is usually also roughly parallel to land surface. This configuration, which is unique to trigonometric leveling, reduces the amount of vertical density gradients the EDM travels through, which, in turn, reduces the uncertainty of the applied refraction correction. Atmospheric conditions are also temporally variable, so F1 and F2 measurements should be made rapidly so the vertical refraction field does not change during the observation period (Ogundare, 2016).

Table 4. Estimated measurement uncertainty caused by Earth's curvature and atmospheric refraction for foresight (slope) distances of $100,200,500,1,000,2,500,5,280$ and 10,560 feet.

[Correction values are based on a total station that is inclined 1 degree above the horizontal plane of the instrument, or a zenith angle of 89 degrees. VD, vertical difference; - , negligible]

\begin{tabular}{rccccc}
\hline $\begin{array}{c}\text { Foresight dis- } \\
\text { tance, } \\
\text { in feet }\end{array}$ & $\begin{array}{c}\text { Correction for Earth's } \\
\text { curvature, } \\
\text { in feet }\end{array}$ & $\begin{array}{c}\text { Correction for } \\
\text { atmospheric refraction, } \\
\text { in feet }\end{array}$ & $\begin{array}{c}\text { Combined correctionfor } \\
\text { curvature and refraction, } \\
\text { in feet }\end{array}$ & $\begin{array}{c}\text { Measured VD, } \\
\text { in feet }\end{array}$ & $\begin{array}{c}\text { Adjusted VD, } \\
\text { in feet }\end{array}$ \\
\hline 100 & 0.0002 & - & 0.0002 & 1.7452 & 1.7450 \\
200 & 0.001 & 0.0001 & 0.0008 & 3.4905 & 3.4897 \\
500 & 0.006 & 0.0009 & 0.0051 & 8.7262 & 8.7211 \\
1,000 & 0.0239 & 0.0034 & 0.0206 & 17.4524 & 17.4319 \\
2,500 & 0.1495 & 0.0214 & 0.1287 & 43.6310 & 43.5028 \\
5,280 & 0.667 & 0.0953 & 0.5740 & 92.1487 & 91.5770 \\
10,560 & 2.668 & 0.3811 & 2.2960 & 184.2974 & 182.0105 \\
\hline
\end{tabular}


The most effective way to reduce uncertainty from curvature and refraction for trigonometric levels is to establish leveling lines that are less than $500 \mathrm{ft}$ because both error sources are distance weighted and are negligible for shorter measurements (tables 4 and 5; USACE, 2007). Regardless of measurement distance, quality meteorological instruments (barometers and thermometers) that are calibrated against a known standard must be used to precisely measure air temperature, barometric pressure, and relative humidity conditions at the station and foresight locations. These observations should be entered into the total station's integrated microprocessor or external data collector (collectively referred to herein as data-collection device) so a refraction correction can be determined and automatically applied to subsequent measurements (fig. 20B). The operator must also ensure that the total station is applying a correction to the EDM, which accounts for Earth's curvature. The procedure to apply curvature and refraction corrections can typically be found in the total station operation manual.

\section{Prism Offset}

Manufactures of survey prisms recognize that even precision retroreflectors have a fixed uncertainty related to the construction of the instrument. More specifically, the uncertainty is caused by the placement, or offset, of the prism in the prism housing by the manufacturer during construction. Typical prism offsets are $0,-17.5 \mathrm{~mm}(-0.0574 \mathrm{ft})$, $-30 \mathrm{~mm}(-0.0984 \mathrm{ft}),-34 \mathrm{~mm}(-0.1115 \mathrm{ft})$, and $-40 \mathrm{~mm}$ $(-0.1312 \mathrm{ft})$. Prism manufacturers and vendors have reported the uncertainty caused by this construction defect as $\pm 1 \mathrm{~mm}$ $(0.00328 \mathrm{ft})$ for precision retroreflectors (Berntsen, 2017). Essentially, the prism offset uncertainty causes the measured slope distance to be slightly longer or shorter than the true distance, which has implications on the VD. For example, the effect of this uncertainty on the derived VD for a measured slope distance of $500 \mathrm{ft}$ and a zenith angle of $60^{\circ}$ is about $\pm 0.0016 \mathrm{ft}$ (table 2). The effect of this uncertainty is proportional to the cosine of zenith angle, so as the zenith angle approaches $90^{\circ}$, the uncertainty becomes negligible. For a prism uncertainty of $1 \mathrm{~mm}(0.00328 \mathrm{ft})$, the VD error caused by the prism error must range from 0 to $1 \mathrm{~mm}$ ( 0 to $0.00328 \mathrm{ft}$ ) for all zenith angles.

The transmitted signal from the EDMI can diverge from the ideal flight path after the signal is reflected from the prism, which is identified by prism manufacturers and vendors as a potential error source called beam deviation (Berntsen, 2017). For poorly constructed prisms, beam deviation may cause the reflected signal to miss the EDMI receiver lens system completely. The theoretical design of the prism is intended to focus the signal on a photo detector in the EDMI for processing (Rüeger, 1990). Manufacturers of precision retroreflectors have reported that an acceptable uncertainty for beam divergence is $\pm 5^{\prime \prime}$ (Berntsen, 2017). Measurement uncertainty that occurs from beam deviation only occurs at the maximum range of the EDMI, which is around 2,500 $\mathrm{m}(8,202 \mathrm{ft})$ for modern total station instruments, so beam deviation is not included as a potential error source in table 2 .

\section{Pointing Error}

Pointing error is the horizontal and zenith angle uncertainty associated with aligning the crosshair of the telescope with the center (apex) of the retroreflector. It is a random error that can be affected by the optical qualities of the telescope and retroreflector, atmospheric conditions (refraction of natural radiation), and the vision of the instrument operator (Ogundare, 2016). The following equation, which has been modified from Chrzanowski (1977), can be used to estimate angular uncertainty from a pointing error:

$$
\sigma p=45^{\prime \prime} \div M
$$

where

$\sigma p \quad$ is the pointing error, in arc-seconds; and

$M \quad$ is the telescope magnification

(dimensionless).

The most effective way to reduce angular uncertainty from pointing error is to increase the telescope magnification $(M)$, which is typically $\times 30$ to $\times 45$ or greater. Because pointing error is a distance-weighted uncertainty that is trigonometrically proportional to the measured slope distance and zenith angle, the effect of pointing error with a $\times 30$ magnification telescope on the derived VD for measurement (slope) distances of 200,500 , and $1,000 \mathrm{ft}$, is $0.0014,0.0035$, and $0.0070 \mathrm{ft}$, respectively (table 2 ). If the telescope magnification is increased to $\times 45$, the VD error resulting from pointing uncertainty for these measurement distances is reduced to $0.0009,0.0023$, and $0.0046 \mathrm{ft}$, respectively. Keeping measurement distances under $500 \mathrm{ft}$, observing angles in F1 and F2, and making repetitive measurements can also reduce pointing error.

Many modern total stations are equipped with ATR, which uses electromagnetic, optical, or both sensing technologies and use advanced algorithms to detect, track, and precisely point the instrument at the center of the target (Leica Geosystems, 2015). This technology can also help reduce measurement uncertainty from pointing errors; however, if the ATR is not calibrated, F1 and F2 observations are required because the pointing error in the F1 observation cancels out the error in the F2 observation because they are equal, although in opposite directions.

\section{Plummet (Centering)}

Plummet error is an instrument defect that causes misalignment of the optical axis of the plummet (optical or laser) and the vertical axis of the total station (Ogundare, 2016). The source of this uncertainty is usually from a plummet or tribrach that is not properly adjusted. Plummets also have an 
Table 5. Recommendations for trigonometric levels.

$\left[\leq\right.$, less than or equal to; EDM, electronic distance measurement; \pm , plus or minus; ${ }^{\circ} \mathrm{C}$, degree Celsius; NGS, National Geodetic Survey; $\geq$, greater than or equal to; F1, direct instrument orientation; F2, reverse instrument orientation]

\begin{tabular}{|c|c|}
\hline $\begin{array}{l}\text { Surveying equipment, prepara- } \\
\text { tion procedure, or trigonometric- } \\
\text { leveling survey procedure }\end{array}$ & Specification, schedule, or criteria \\
\hline Total station angular precision & $\leq 5$ arc-seconds \\
\hline EDM uncertainty & $\leq 3$ millimeters and 3 parts per million \\
\hline $\begin{array}{l}\text { Data collector or onboard micro- } \\
\text { processor }\end{array}$ & Direct and reverse measurement processing; coordinate geometry and stakeout programs \\
\hline Surveying prism & $\begin{array}{l}\text { Quality optical glass with known offset; beam divergence } \leq 2 \text { arc-seconds; } \leq 1 \text {-millimeter prism-offset } \\
\text { uncertainty }\end{array}$ \\
\hline Plummet & $\leq 2.5$-millimeter centering uncertainty \\
\hline Tripod & $\begin{array}{l}\text { Adjustable center pole with fixed height dowel stops; or adjustable tripods with a dual clamp locking } \\
\text { mechanism; tripods should have high torsional rigidity. }\end{array}$ \\
\hline Tribrach & High torsional rigidity \\
\hline Thermometer & Uncertainty of $\pm 1^{\circ} \mathrm{C}$ \\
\hline Barometer & Uncertainty of \pm 0.1 inch mercury \\
\hline Optical level bubble adjustment & Daily ${ }^{1}$ \\
\hline Digital level bubble adjustment & Daily 1,2 \\
\hline Plummet adjustment & Daily ${ }^{1}$ \\
\hline Vertical collimation & Daily ${ }^{1}$ \\
\hline Horizontal collimation & Daily 1 \\
\hline $\begin{array}{l}\text { EDM test on NGS calibrated } \\
\text { baseline }\end{array}$ & Annual \\
\hline $\begin{array}{l}\text { Meteorological observations for } \\
\text { curvature and refraction cor- } \\
\text { rections }\end{array}$ & Uncertainty of $\pm 1^{\circ} \mathrm{C}$ for temperature and \pm 0.1 inch mercury for barometric measurements \\
\hline Instrument-height measurements & $\begin{array}{l}\text { Ensure slant height measurement is reduced to vertical equivalent (fig. 19); redundant height measure- } \\
\text { ments; consider use of tripod with adjustable center pole with fixed-height dowel stops. }\end{array}$ \\
\hline Establish origin point & $\begin{array}{l}\text { To establish a new survey marker, see Federal Geodetic Control Committee (1984), Kenney (2010), } \\
\text { Smith (2010), and Rydlund and Densmore (2012); to recover a survey marker, see the National } \\
\text { Geodetic Survey's Integrated Database. }\end{array}$ \\
\hline Establish datum & $\begin{array}{l}\text { Differential leveling procedures described by Kenney (2010); or trigonometric-leveling procedures } \\
\text { described in this manual; or satellite-based methods described by Rydlund and Densmore (2012) }\end{array}$ \\
\hline Establish baseline & $\begin{array}{l}\text { Average derived vertical difference and horizontal distance from three F1 and three F2 measurements. } \\
\text { Orientation can be established with Global Navigation Satellite System, compass, or assumed. }\end{array}$ \\
\hline F1 and F2 observations ${ }^{3}$ & $\begin{array}{l}\text { Measurement sequence must include backsight and foresight observations for traverse surveys; side- } \\
\text { shots are observed to the foresight only. }\end{array}$ \\
\hline Reciprocal measurements ${ }^{3}$ & Must be observed for each leveling leg or section for traverse surveys \\
\hline Measurement distances ${ }^{3}$ & $\leq 500$ feet $^{4}$ \\
\hline Backsight check ${ }^{5}$ & $\mid \leq 0.030$ foot $\mid$ \\
\hline
\end{tabular}

1Procedures are completed on the day of the campaign before the start of the survey.

2Electronic bubbles are required to be checked multiple time per day when air temperatures changes exceed 20 degrees Fahrenheit.

${ }^{3}$ See table 9 for measurement specifications.

${ }^{4}$ See table 9 for exceptions to the measurement distance limit.

${ }_{5}^{5}$ Measurement for backsight check observed in F1 only. 
inherent uncertainty that can range from less than $1.8 \mathrm{~mm}$ to $2.5 \mathrm{~mm}$ for even the most precise instruments (Dracup and others, 2019). However, since this error is only in the direction of the horizontal plane of the instruments (total station and prism) it will not influence the VD of the measurement if the instruments are level. To ensure the instrumentation is level and centered, procedures to adjust the level bubble and the plummet are described in the "Preparing for Trigonometric Leveling" section. Once these adjustments have been made, the measurement uncertainty from plummet error is negligible.

\section{Reading Error}

Measurement uncertainty from reading error is caused by the inability of the operator to repeat the same reading (Ogundare, 2016). To reduce the angular uncertainty from this random error source, multiple sets of zenith angles should be observed in F1 and F2 (table 2). Observing, then averaging, repetitive angles in F1 and F2 is the most effective way to reduce VD uncertainty caused by reading error. The datacollection device can be programmed to average zenith angles (and slope distances) from multiple observation sets.

\section{Compensator-Index Error}

Modern total stations are equipped with dual-axis compensators that correct the horizontal and zenith angles when the instrument is not perfectly level and the telescope is tilted from the horizontal plane of the instrument (Cothrun, 1995). Compensator-index error is the residual error after the dualaxis compensator applies a correction to the horizontal and zenith angle measurements (Ogundare, 2016). The effect of this error source on the VD can be reduced or eliminated by observing measurements in F1 and F2 (table 2).

\section{Meteorological Measurements (Temperature and Pressure)}

Air temperature, barometric pressure, and relative humidity measurements must be made using quality meteorological instruments that have been calibrated to a known standard. Erroneous corrections for atmospheric refraction will be applied to the EDM if meteorological measurements are not accurate or incorrectly entered into the data-collection device. For example, an error of $1{ }^{\circ} \mathrm{C}\left(1.8^{\circ} \mathrm{F}\right)$ could cause a distanceweighted error of $0.8 \mathrm{ppm}$ for infrared distance measurements (USACE, 2007). The effect of a $0.8 \mathrm{ppm}$ error on the VD is $\pm 0.0002 \mathrm{ft}$ for a zenith angle of $60^{\circ}$ and a measurement distance of $500 \mathrm{ft}$ (table 2). The effect of a barometer that is accurate to $\pm 3 \mathrm{~mm}( \pm 0.118$ inch [in.]) of mercury on the $\mathrm{VD}$, measured at the same angle and distance, is $\pm 0.0003 \mathrm{ft}$. Barometers should be accurate to 0.1 in. (USACE, 2007).
Meteorological observations should be made at the locations of the total station and the foresight prism (near the objective survey marker) to detect changes in atmospheric conditions along the measurement path. If significant changes are detected - indicated by meteorological observation differences between the station and foresight locations that exceed the error threshold of the meteorological instrument - the operator should calculate an average condition along the measurement path and enter it into the data-collection device so a refraction correction can be determined and automatically applied to subsequent measurements (fig. 20B). The procedure to enter meteorological observations into the datacollection device can typically be found in the instrument's operation manual. Relative humidity, wind speed, cloud cover, and visibility should also be measured and documented. Meteorological instruments should be calibrated according to the procedures and schedule recommended by the manufacturer. If erroneous measurements are detected or indicated, the surveyor should not use the instrument until it has been field calibrated against a known standard or shipped back to the manufacturer or vendor for a laboratory calibration.

\section{Other Sources of Uncertainty}

The orientation of a tribrach relative to its vertical axis can be altered by torsional forces during a trigonometric levels survey (Leica Geosystems, 2010). When a torque is applied to the total station as it is pointed at a target, a small torsional force occurs in the tribrach. During a survey, after many measurements are made from the same station, the orientation of the tribrach can change, which may cause erroneous angle measurements. For trigonometric-leveling surveys, the amount of applied torque is reduced because only a few measurements are made per station, as compared to design surveys when many measurements may be made. Precision tribrachs can absorb the force without lasting deformation, so it is important to know the torsional rigidity of the tribrachs that are being used for a trigonometric levels survey. Like quality tripods, it is important to select tribrachs with high torsional rigidity so that an applied torque does not affect the quality of the measurement.

Plate bubble and standing-axis (vertical-axis) errors are not a substantial part of the VD error budget because dual-axis compensators correct angular measurements for the effect of these systematic error sources (table 2). Random leveling errors are also corrected by the dual-axis compensator.

\section{Trigonometric Leveling}

Three common types of trigonometric-leveling techniques have been described in several literature sourcesunidirectional leveling, leap-frog (forced-centering) leveling, and reciprocal leveling (Chrzanowski, 1989; Ceylan and others, 2005; USACE, 2007). The simplest leveling technique 
is unidirectional leveling, which requires a tripod-mounted total station at the station and a tripod-mounted prism at the foresight. The VD between the horizontal plane of the instrument and the horizontal plane of the prism is determined by multiplying the measured slope distance by the cosine of the zenith angle (fig. 8). Averaging multiple observations made in F1 and F2 is critical for obtaining quality results using this procedure. This procedure is common for applications such as establishing a baseline or obtaining the last measurement of a closed traverse because it is a fast and effective way to determine the VD and horizontal distance between two points.

Leap-frog, or forced-centering, leveling requires a tripodmounted total station set up at the station with tripod-mounted prisms at the backsight and foresight. After a measurement set is completed, typically consisting of a set of four measurements (two F1 and two F2), the total station is unlocked from the tribrach and advanced to the foresight. The prism at the foresight is unlocked from the tribrach and moved back to the station. The prism assembly and tripod at the backsight is set up above the newly established survey marker (objective point) and is considered the foresight for the next setup. Quality tribrachs with precisely adjusted level bubbles are critical for this procedure to ensure precise centering and level over the ground point. The advantage of this method is the surveying instruments that have been exchanged at the backsight and station (prism and total station, respectively) do not have to be releveled or recentered over the survey marker because the existing tribrach (if properly adjusted) and tripod assemblies "force" these conditions on the equipment.

However, both centering and leveling should be verified at the backsight, station, and foresight by the survey crew to ensure a quality setup.

Reciprocal leveling involves determining an elevation of a survey marker from two or more locations and averaging the solution. Simultaneous reciprocal leveling is typically used for long measurement distances to offset the effects of atmospheric refraction; two total stations are required, which are set up at each end of the measurement line, that make concurrent observations in opposite (reciprocal) directions. Reciprocal observations that are made with a single total station are considered "near-simultaneous" if observations are made within a short period and substantial time does not elapse. The trigonometric-leveling techniques that are described in this manual integrate components of all three methods to accurately determine VDs and are described in the following sections.

\section{General Specifications}

During trigonometric-leveling campaigns, survey preparation requirements involve daily inspection, and if necessary, adjustments of digital and optical levels, and optical and laser plummets. Electronic bubbles are required to be checked, and adjusted if necessary, multiple times per day when diurnal air temperatures changes exceed $20^{\circ} \mathrm{F}$ (Paiva, 2016). Horizontal and vertical collimation tests should also be conducted daily during a trigonometric-leveling campaign to determine and correct for measurement uncertainty due to axial defects (table 5). The EDMI should be tested on NGS calibrated baselines at least once a year and as conditions warrant. A tripod equipped with an adjustable center pole with fixed-height dowel stops is recommended to obtain quality instrumentheight measurements (HI and $\mathrm{SH}$ ). If an adjustable-height tripod is being used, determine the $\mathrm{HI}$ and $\mathrm{SH}$ with the method described in the "Sources of Measurement Uncertainty for Trigonometric Leveling" section (fig. 19). Additional information on preparation procedures and best practices are described in the "Preparing for Trigonometric Leveling" and the "Sources of Measurement Uncertainty for Trigonometric Leveling" sections of this manual and are summarized in table 5. Subsequent sections of this manual describe the requirements for running trigonometric levels in the USGS and are also summarized in table 5 .

Recommended specifications for trigonometric-leveling equipment include a total station instrument with an angular precision of less than or equal to 5" equipped with an EDMI having a measurement uncertainty of less than or equal to $3 \mathrm{~mm}$ plus $3 \mathrm{ppm}$ (table 5). The distance-weighted component of the EDM uncertainty is less important than the constant component because measurement distances should be less than $500 \mathrm{ft}$. The data-collection device should have the capability to average multiple sets of F1 and F2 measurements, and coordinate geometry and stakeout programs, which are standard for most surveying software packages. Additional equipment recommendations are described in the "Trigonometric-Leveling Equipment" and the "Sources of Measurement Uncertainty for Trigonometric Leveling" sections of this manual and are summarized in table 5.

Backsight check is a quality-assurance procedure used to verify station setup information; this information includes the point numbers, positions, orientation, and instrument heights of the station and backsight. After the observation for the backsight check is made, the data-collection device displays a residual value (sometimes called the "delta") for the horizontal and vertical (elevation) components; the residual value is the difference between the known and measured coordinate of the backsight. If the $\mathrm{HI}$ and $\mathrm{SH}$ are accurate, the uncertainty of the backsight check is usually caused by the observed zenith angle. For this reason, some data-collection devices have automated routines that allow the user to conduct a backsight check using F1 and F2 measurements which may reduce the residuals. If a F1-only measurement is used for the backsight check, the residual value for the vertical component should not exceed $\pm 0.03 \mathrm{ft}$; if $\mathrm{F} 1$ and $\mathrm{F} 2$ measurements are used, the residual value for the vertical component should not exceed $\pm 0.015 \mathrm{ft}$. The backsight check is strictly a quality-assurance procedure and should not be used to modify the raw field data. 


\section{Establishing an Origin Point for the Survey}

The first objective of a trigonometric levels survey is to establish an origin point, which is a new or existing survey marker typically referenced to some assumed, local, tidal, or terrestrial datum (fig. 21). If a new mark is established, it should be a benchmark set in a stable location that is not subject to land-surface motion, potential land development, vehicle and heavy foot traffic, vandalism, or other hazards that can potentially damage or destroy the mark. The benchmark location should have an unobstructed line of sight in the direction the survey will progress and should be easy to access and recover. Detailed methods for establishing benchmarks are described by Federal Geodetic Control Committee (1984), Kenney (2010), Smith (2010), and Rydlund and Densmore (2012).

If an existing mark is recovered, the surveyor should ensure that it is a fiducial benchmark from a trusted agency such as the NGS, USGS, and NOAA. The NGS maintains an Integrated Database, which provides datasheets for survey markers published in the National Spatial Reference (NOAA, 2018). An interactive mapper can be used to identify and determine the location of trusted benchmarks. Benchmark elevation, source datum, applied adjustments, stability and recovery history, and other metadata should be reviewed and verified before the mark is selected as the origin point for the survey. Because the accuracy of a trigonometric-leveling survey depends on the accuracy of the origin point, recovering or establishing a quality survey marker is a critical first step.

\section{Establishing a Datum}

Referencing a trigonometric-leveling survey to a geodetic datum is desirable and is often mandated by project requirements and the cooperating agency. For example, at USGS streamgaging stations that are used for flood forecasting by the National Weather Service, perpetuated elevations from trigonometric levels may need to be referenced to the North American Vertical Datum of 1988 (NAVD 88) for modeling purposes (Kenney, 2010). In some cases, an assumed, local, or tidal datum may be used as the origination datum for a trigonometric-leveling survey, which can be subsequently translated to a geodetic datum. Regardless of the origination datum, NAVD 88 is the current vertical datum for the United States and thus it is critical to recover fiducial benchmarks with published NAVD 88 values. An interactive mapper maintained by the NGS can be used to identify trusted benchmarks (in the NGS Integrated Database) established during the general adjustment of the NAVD 88 (NOAA, 2018). Information for these passive (and active) monuments are described in datasheets and can be downloaded as geographic information system shapefiles. If the project requires a datum other than assumed, it is best practice to establish the datum immediately before or during a trigonometric-leveling survey because survey markers established during the campaign can be subsequently lost, damaged, or obliterated. Establishing the datum on the origin point will ensure all subsequent measurements are also tied to the datum.

There are two approaches for tying a trigonometricleveling survey to NAVD 88-a terrestrial approach, which requires running a traditional survey (differential or trigonometric) from a trusted benchmark, and a satellite-based approach, which requires establishing a trusted benchmark using a survey-grade (centimeter-level accuracy) Global Navigation Satellite System (GNSS) (fig. 21). At the time of this writing (2020), both approaches require recovering fiducial benchmarks with published NAVD 88 values (NOAA, 2018). Methods for perpetuating the datum from trusted benchmarks to the project area using differential leveling methods are described by Schomaker and Berry (1981) and Kenney (2010). Trigonometric leveling can also be used in lieu of, or in combination with, differential leveling to tie an existing trigonometric levels survey to a datum if the rigorous protocol proposed in this manual is implemented.

A hybrid survey uses terrestrial- and satellite-based surveying methods to establish and perpetuate vertical control. In most cases, a GNSS is used to establish the datum and to georeference and orient the survey; trigonometric leveling, or another terrestrial leveling method, is then used to perpetuate elevations to objective survey markers. If a hybrid survey approach is implemented and a GNSS will be used to measure the origin point, the surveyor should avoid reflective objects such as large buildings, signboards, chain-link fences, water towers, and power and transmission lines (Rydlund and Densmore, 2012). The benchmark should be set in a location with southern atmospheric exposure (in the northern hemisphere) that is free of tree canopy and other vegetation.

\section{North American-Pacific Geopotential Datum of 2022}

The NAVD 88 is the current vertical datum for the United States and is based on the height of the primary tidal benchmark that represents local mean sea level at Father Point/Rimouski, Quebec, Canada (Martin, 2015). This datum cannot be maintained or perpetuated by the NGS because it is based on passive, terrestrial monuments that are not regularly checked for movement, are not spatially distributed, and are vulnerable to disturbance or destruction (NOAA, 2007).

In 2007, the NGS started work on the Gravity for the Redefinition of the American Vertical Datum (GRAV-D) project that models and monitors the surface of the Earth's gravity field (geoid) for improving how elevations (heights) are determined in the United States and its territories (Smith and Roman, 2010). At the time of this writing (2020), a future geopotential datum resulting from the GRAV-D project is anticipated in 2022 and has been tentatively identified as North American-Pacific Geopotential Datum of 2022 (NAPGD2022) by the NGS (Rydlund and Noll, 2017). NAGPD2022 will replace the current vertical datum of the United States with 


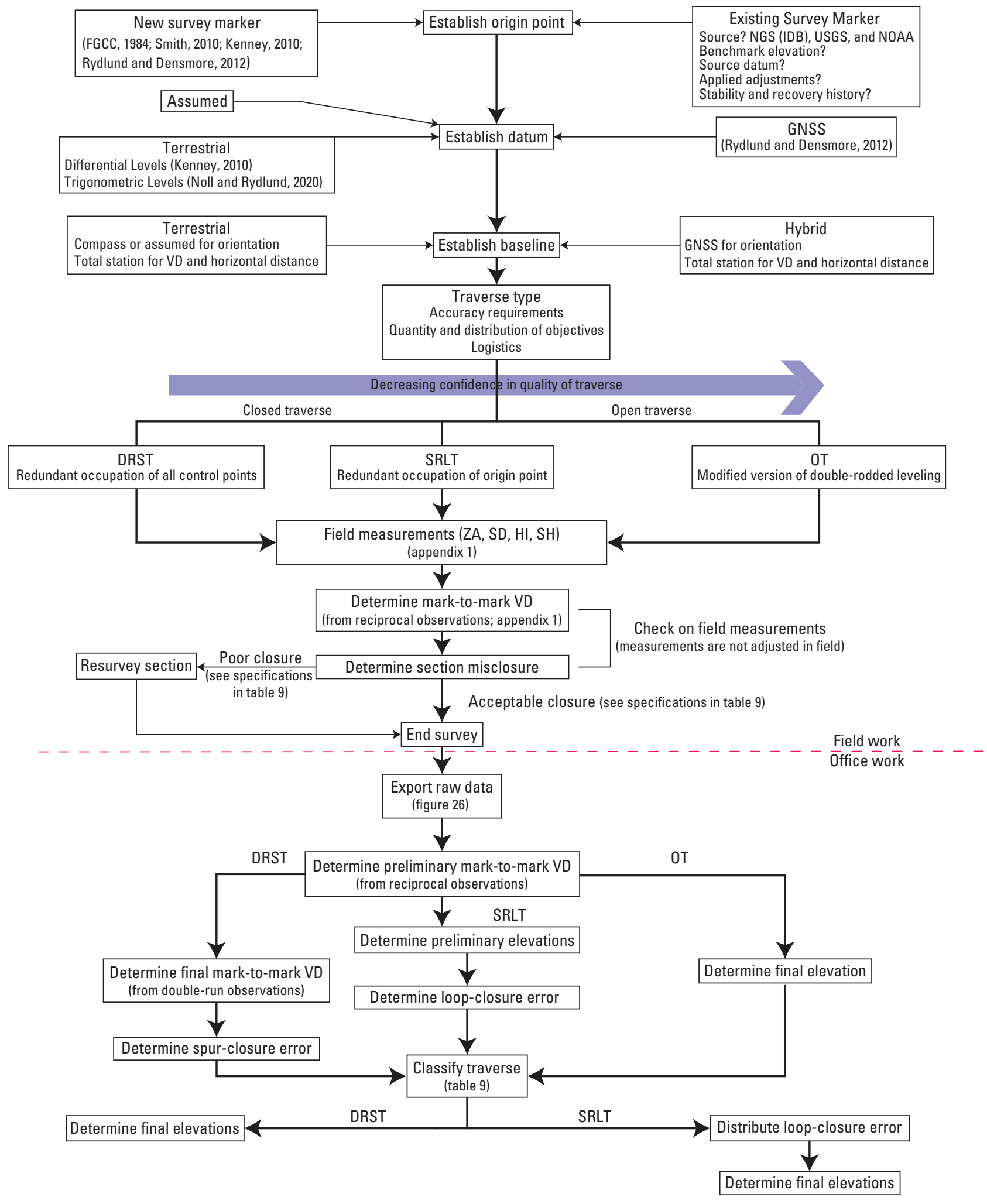

Figure 21. The trigonometric levels decision tree and survey process. 
a new gravity-based, geopotential datum that references a gravimetric geoid accurate at the $0.033 \mathrm{ft}$ level (Childers and Youngman, 2016). Future versions of the National Geodetic Survey software utilities VDatum and VERTCON are anticipated to provide a transformation of NAVD 88 elevations to the new geopotential datum. These conversion tools will be critical to translate vertical control originally established in NAVD 88 (or other) to the new geopotential datum. If a new trigonometric-leveling survey is required to reference NAGPD2022, the fiducial benchmark will be measured with GNSS to obtain the ellipsoid height; then, the new gravimetric geoid will be applied to ellipsoid height to obtain the orthometric height in NAGPD2022. More information on datum conversions is available in Rydlund and Noll (2017).

\section{Establishing a Baseline}

Once an origin point has been determined for a trigonometric-leveling survey, a baseline, or a reference line segment of known direction and distance between the endpoints, must be established to set the orientation of the survey and begin leveling (fig. 21). Procedures to establish a foresight survey marker (or any monumented or non-monumented benchmark), which is considered one of the endpoints that define the baseline (the other endpoint is the origin point), are described by FGCC (1984), Kenney (2010), Smith (2010), and Rydlund and Densmore (2012). Unidirectional trigonometric leveling is the preferred surveying method to transfer an elevation from the origin point to the foresight point along a baseline (Ceylan and others, 2005). Instrument operators should use a data-collection device to avoid transcription errors, to automate the data collection process, and to ensure corrections for curvature and refraction along the baseline. Although observed horizontal angles are not critical to running a trigonometric-leveling survey, surveyors should accurately determine the horizontal coordinates for each survey marker, which should be referenced to a horizontal datum (assumed, local, and so on), in case the survey lines need to be retraced. Once the coordinates of the baseline endpoints are established and entered into the data-collection device, horizontal coordinates will be automatically computed and stored for future foresight survey markers (objective points) if the datacollection device is properly configured. Horizontal coordinates are computed using coordinate geometry programs that convert the observed horizontal angle and slope distance measured to the foresight, to a position relative to the total station. Careful accounting of the station and backsight locations in the data collector is critical for determining correct horizontal positions. Some total stations also require that the horizontal angle reading is zeroed when pointed at the backsight target to verify the station setup. Using a data-collection device has automated and simplified the process of establishing a baseline, so operators should consult their user manual for the procedure that is specific to their equipment. The following procedure is purposely generalized so it can be applied to all equipment models of varying age, make, and model.

After trigonometric-leveling equipment has been prepared using the procedures described in the "Preparing for Trigonometric Leveling" section, which includes techniques to adjust the level bubbles and optical plummets of the total station and tribrachs, the operator will set up, level, and precisely center the total station over the origin point. As a rule of thumb, the height of the telescope should be set to the height of the operator's eye, but this must be considered in view of the highest and lowest zenith angle readings that will be observed. Measurements that require steep sightings may not be feasible if the eyepiece is at the level of the observer's eye when the telescope is horizontal.

The USACE (2007) reports combined uncertainty from $\mathrm{HI}$ and $\mathrm{SH}$ measurements can be up to $\pm 0.1 \mathrm{ft}$, so redundant height measurements are required when establishing a baseline and should be within $|\leq 0.005| \mathrm{ft}$. Measure the HI using the previously described methods (fig. 19) and record the average height from the redundant measurements. Once the HI has been determined, set up, level, and center the tripod and prism assembly over the foresight survey marker. Average and record the SH from the redundant measurements. If a tripod with an adjustable center pole with fixed-height dowel stops is being used to establish the baseline, verify the center-pole height with a single measurement. After the HI and SH have been determined, make meteorological observations with instruments that have been calibrated to a known standard at the origin point and foresight survey markers. If air temperature, barometric pressure, and relative humidity measurements vary substantially between the endpoints of the baseline, calculate an average for each observation. These observations should be entered into the data-collection device (integrated microprocessor or external data collector, but never both) so a correction for atmospheric refraction can be determined and automatically applied to subsequent measurements (fig. 20B). Ensure the data-collection device is configured to apply corrections for the curvature of the Earth, which should be insignificant for measurement distances of less than $500 \mathrm{ft}$.

Configure the digital display on the data-collection device to show the VD. Using the prism target plates as a reference, precisely point the total station at the center (apex) of the prism at the foresight survey marker and take a measurement. Record the VD that is shown on the display, then repoint the total station in F2 and take another measurement to the same prism at the foresight point. Repeat this process about three times in F1 and three times in F2, documenting the derived VD in a field book after each measurement. Calculate the average VD from the six measurements, making sure to account for the sign of the VD (positive or negative). A positive VD indicates the horizontal plane of the prism is higher than the horizontal plane of the instrument; conversely, a negative VD means the instrument is higher than the prism (fig. 8). Once the VD, the heights of the total station and prism, and 
the elevation of the origin point survey marker are known, use the following equation to calculate the elevation of the foresight survey marker:

$$
e B M 2=e B M 1+H I+V D-S H_{f}
$$

where

$e B M 2$ is the elevation of the foresight survey marker, $e B M 1 \quad$ is the elevation of the origin point survey marker,

$H I \quad$ is the instrument height above $B M 1$,

$V D \quad$ is the average of the derived VD from at least six total station measurements made in $\mathrm{F} 1$ and $\mathrm{F} 2$ ( $V D$ can be positive or negative), and

$\mathrm{SH}_{f} \quad$ is the prism height above $B M 2$ or foresight. If highly accurate horizontal orientation is required for the survey, the surveyor can determine the azimuth of the baseline by tying it to existing horizontal control benchmarks from NGS (NOAA, 2018) or other trusted sources; or if using a hybrid survey approach, take a GNSS measurement on both endpoints of the baseline and use coordinate geometry (inverse tool) to calculate the azimuth between the points (fig. 21). If only coarse baseline orientation is required for the survey, a compass observation can be made from the origin point to the foresight to determine the direction of the baseline. If the project requires a more accurate horizontal orientation later, the baseline azimuth can be refined with a geometric rotation around a fixed point.

The next step is to determine the horizontal distance of the baseline by configuring the digital display to show a derived horizontal distance (fig. 8). The horizontal distance can be determined using the same method as the VD, by taking the average horizontal distance from three F1 observations and three F2 observations - this can be done concurrent with the VD measurements by toggling the display to show the VD, then the horizontal distance, after each measurement. Once a direction and distance have been determined from the origin point to the foresight, enter the XYZ coordinates of the foresight survey marker into the data collector. For example, if the foresight survey marker is $414.22 \mathrm{ft}$ (determined from the total station) from an origin point along a generally northeast azimuth of $53^{\circ} 00^{\prime} 00^{\prime \prime}$ (determined from the compass), and the origin point of the survey is assumed to be at $5,000 \mathrm{ft}$ north and 5,000 ft east of the origin of a Cartesian coordinate system (where the $\mathrm{x}$-axis intersects the $\mathrm{y}$-axis at 0,0 ), the horizontal coordinate for the foresight is $5,249.28 \mathrm{ft}$ north and $5,330.81 \mathrm{ft}$ east. These coordinates can be translated from a Cartesian system to a geographic coordinate system (or other) later using the coordinate geometry functionality of the data-collection device (rotations and translations) or during postprocessing. Once the XYZ coordinates of the baseline endpoints have been determined and entered into the data-collection device, the surveyor can begin the trigonometric-leveling survey.

\section{Direct and Reverse Instrument Orientation Measurements}

Observing zenith angles and slope distances in F1 and $\mathrm{F} 2$ is the most important procedure for precisely determining VDs between the horizontal plane of the instrument and the horizontal plane of the prism (fig. 8). One set of F1 and F2 measurements consists of four observations - two of these observations are made on the backsight, the first in F1 and the second in F2; and the remaining two observations are made on the foresight, the first in F1 and the second in F2. A typical four-measurement sequence (set) consists of (1) the backsight observed in F1, (2) the foresight observed in F1, (3) the foresight observed in F2, and (4) the backsight observed in F2. The instrument telescope is inverted between the second and third measurement, which is the critical step of this procedure because the F1 and F2 observations are averaged, reducing or eliminating measurement uncertainty from error sources such as vertical collimation, horizontal collimation, and compensator index (Ogundare, 2016). Additionally, because this procedure requires repetitive measurements, uncertainty from pointing error is also reduced. Random error from the EDM observations will also be reduced because slope distances observed in F1 and F2 are averaged to determine measurement distance. The operator should use the coordinate geometry programs on the data-collection device to average F1 and F2 observations so that transcription errors are minimized, and calculations are automated. A second set of observations may be required if the difference between the F1 and F2 measurements exceed the angular error threshold. It may be possible to set angle and distance thresholds in the data-collection device to warn the operator if a limit is exceeded. The following equations can be used to calculate the mean slope distance and zenith angle for F1 and F2 measurements:

$$
S D_{m}=(S D[F 1]+S D[F 2]) \div 2 \text { and }
$$

$$
Z A_{m}=(Z A[F 1]+[360-Z A(F 2)]) \div 2,
$$

where

$$
\begin{aligned}
S D_{m} & \text { is the mean slope distance, in feet; } \\
Z A_{m} & \text { is the mean zenith angle, in degrees; } \\
S D & \text { is the slope distance, in feet; } \\
Z A & \text { is the zenith angle, in degrees; } \\
F 1 & \text { is the direct instrument orientation; and } \\
F 2 & \text { is reverse instrument orientation. }
\end{aligned}
$$

Multiple sets of F1 and F2 observations are recommended to improve measurement quality and reduce uncertainty from error sources from the total station, operator, and the environment (table 2). 


\section{Reciprocal Measurements}

Reciprocal observations are used to offset measurement uncertainty due to atmospheric refraction and curvature but, importantly, can be used to determine the leveling quality of each leveling line, which is indicated by the section misclosure. Section misclosure is determined by adding the VD determined in one direction with the VD determined in the reciprocal direction. The F1 and F2 measurement sequence was designed so the backsight and foresight are both observed from each station in the traverse. As a traverse progresses from station to station along the survey route, the foresight measurement made at the previous station is reciprocated with a backsight measurement at the subsequent station (nearsimultaneous observations). The first measurement is made from the previous station to the foresight along a leveling line (section); the second observation is made in the reciprocal direction along the same leveling line, from the subsequent station to the backsight. The VDs of the reciprocal measurements are then documented in a field book or standardized form; the digital display on the data-collection device can be programmed (toggled) to show the VD (fig. 8; app. 1). If the $\mathrm{SH}$ and $\mathrm{HI}$ are equivalent the VD can be used to evaluate the section misclosure (described in a subsequent section); if not, determine the mark-to-mark VD (fig. 8; eq. 8) between the survey markers.

The mark-to-mark VDs between successive control points are calculated in reciprocal directions for each section of the traverse. Perfect closure of a section is indicated when the mark-to-mark VD in one direction is equivalent to the mark-to-mark VD in the reciprocal direction but have opposite signs (more specifically, when the sum of the mark-to-mark VDs is equal to zero). Section misclosure should be documented in the field (app. 1) by the survey crew chief before the instrumentation is advanced to the next station in case the section must be releveled. Real-time evaluation of section misclosure is an important quality-assurance procedure to determine survey quality and detect measurement blunders.

Adjustments to field measurements are typically done in the office by the analyst after the raw data have been downloaded, compiled, and scrutinized. This process is described in the "Adjustments of Field Measurements" section. Section misclosure is an important indicator of measurement quality because it represents the error in the relative height differences between successive control points and not the accumulation of error in the entire traverse. Section misclosure may indicate small systematic errors, or gross measurement blunders, or both; and may help the analyst identify the strongest and weakest sections of the traverse, which will inform decisions about future datum perpetuation from existing control points.

\section{Foresight Distances}

Many sources of error for total station measurements are proportional to the measured slope distance, so it is recommended that foresight distances are less than $500 \mathrm{ft}$ to reduce these uncertainties (table 2). Modern total stations are capable of measuring distances greater than $8,000 \mathrm{ft}$; however, at this range a small distance-weighted uncertainty can become a substantial part of the VD error budget, especially for steep zenith angles. The USACE (2007) recommends foresight distances of less than $1,000 \mathrm{ft}$ to minimize effects from atmospheric refraction $(r)$ and Earth's curvature $(c)$; for distances less than $500 \mathrm{ft}$, measurement uncertainty due to $c$ and $r$ is significantly reduced as these errors are a function of the square of the distance from the instrument to the target, but they should still be accounted for as described in the "Sources of Measurement Uncertainty for Trigonometric Leveling" section.

\section{Traverse Surveys}

A traverse survey is a series of successive straight lines of known distance and orientation that connect a series of points on the Earth (USACE, 2007). Field measurements of survey lines are typically determined with a total station, which uses previously surveyed points as a reference to establish subsequent points. In trigonometric leveling, measured slope distances, zenith angles, and instrument heights are used to determine mark-to-mark VDs between points. Horizontal angles are less critical but are still important for horizontal positioning and if survey lines need to be retraced. A closed traverse originates at a point of known location and terminates on a point of known location (fig. 22). Two types of closed traverses are used to establish vertical-control networks in the USGS: a single-run loop traverse (SRLT) and a double-run spur traverse (DRST; fig. 22). These traverses are considered closed because the survey begins and ends at the fiducial survey marker at the origin point. The term "single run" is used to describe sections of a traverse that are leveled away (in the forward direction) from the origin point and towards the objective point (Schomaker and Berry, 1981). Single-run leveling must form loops with other leveling for the purpose of detecting measurement blunders. Sections that are leveled in the opposite direction are considered backward running. Collectively, the forward and backward direction of leveling is called a "double run." All control points are occupied twice (redundant occupation) in a double-run leveling survey.

Leveling measurements of a SRLT are made in the forward direction from the origin point to the destination and then from the destination to the origin point along a different leveling route. The leveling route of a SRLT resembles a loop or a series of successive leveling sections (legs) that are connected to form a polygon (fig. 22). The closure error of a SRLT, or loop-closure error (LCE), is typically determined by an observation from the last station in the traverse to the origin 


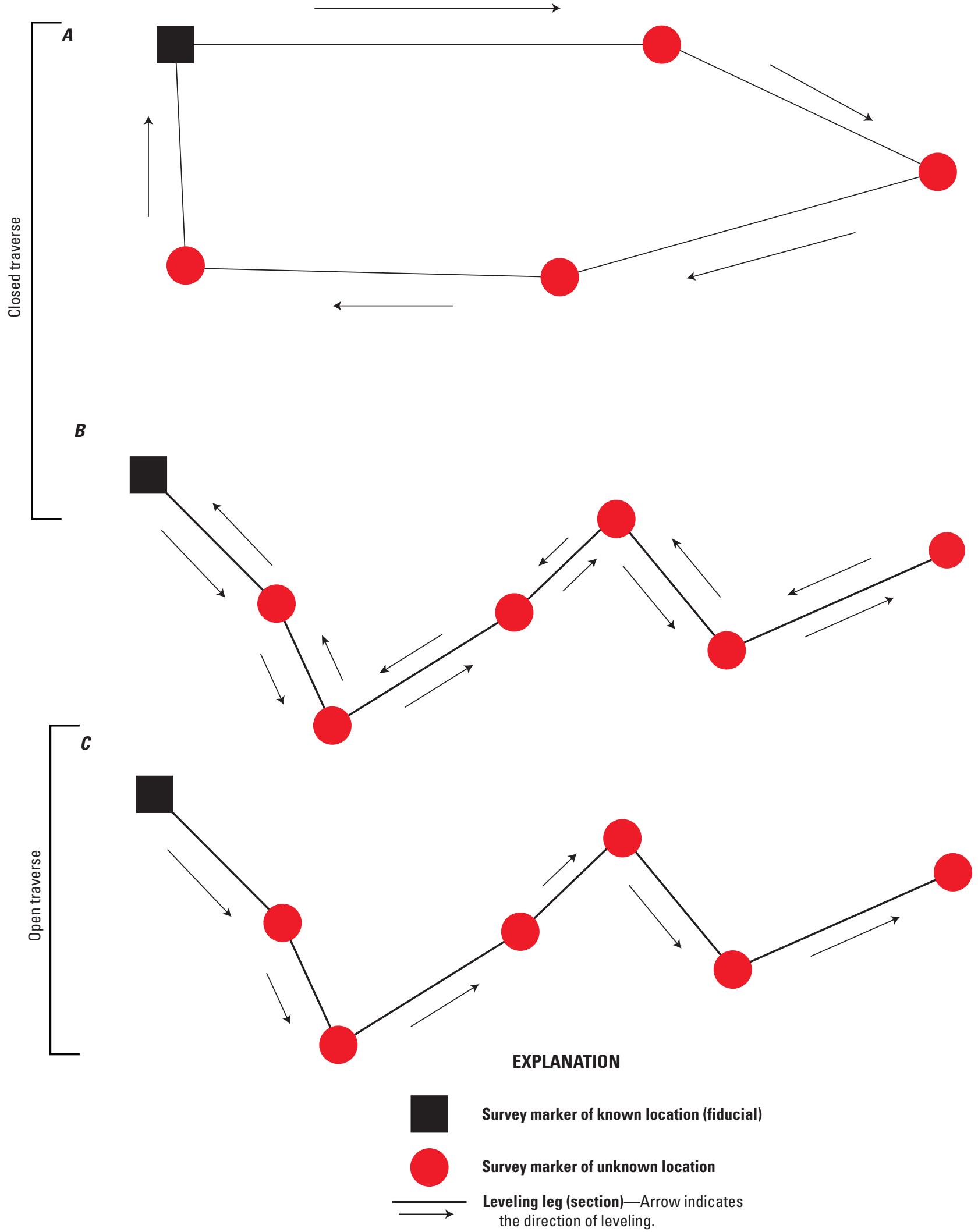

Figure 22. Closed and open traverse surveys. $A$, single-run loop traverse; $B$, double-run spur traverse; and $C$, open traverse. 
point. The origin point is the only point that is redundantly occupied in a loop traverse. The first occupation occurs when the baseline is established, and the second occupation occurs during the last setup of the traverse to evaluate the misclosure of the final section. The LCE is determined by subtracting the final (measured) elevation of the fiducial survey marker from the starting (known) elevation. The LCE may indicate the accumulation of systematic leveling errors during the traverse and is useful to detect measurement blunders. Reciprocal measurements made in F1 and F2 are used to determine section misclosure and adjust field measurements.

Leveling measurements of a DRST are made in the forward direction from the origin point to the destination and then in the backward direction from the destination to the origin point along the same leveling route (fig. 22). Vertical-control points are redundantly occupied during the second (backward) running of the spur, which is used to check the leveling results from the first running in the forward direction (Schomaker and Berry, 1981). The forward and backward leveling of a DRST should be done at different times of the day to ensure environmental conditions have changed. Good practice is to do the forward running in the morning and the backward running in the afternoon (Schomaker and Berry, 1981). The leveling route forms a jagged line, which consists of a series of successive leveling sections that are perpetuated from existing vertical control and typically terminate at a project site or location that requires precise vertical datum for data-collection activities. Leveling lines that are used to transfer the datum from an existing benchmark or vertical-control network to a location not otherwise connected to a network are identified as spurs (Schomaker and Berry, 1981). Reciprocal measurements made in F1 and F2 are used to determine section misclosure and adjust field measurements.

When selecting between closed traverse methods (DRST and SRLT), the surveyor should not only consider the accuracy requirements of the project but also the quantity and spatial distribution (geometry) of the objectives. The redundant occupation of control points from a DRST will generally produce a higher quality solution (final elevation) then a SRLT (single occupation of control points); however, if the control points are distributed irregularly a SRLT may be a viable option, especially if the quality of the final elevations are minimally affected. Conversely, if the distance between the origin and the objective is small or only one objective exists, a DRST could be a practical option because a straight line (as opposed to a polygon) may be the most efficient route between the origin and the objective. Furthermore, if the surveyors need to clear a line of site (cut line) between control points because of heavy vegetation, a DRST reduces the need for extra work because survey lines are retraced to the origin during the backward running. In general, a SRLT should be considered if there are numerous objectives that are spatially distributed in a nonlinear geometry. DRST should be considered if there is a single objective, or numerous objectives that are distributed in a linear geometry. Accuracy requirements, quantity and distribution of objectives, and logistics must be considered when selecting a traverse method to perpetuate or establish the datum at a project site. Loop and spur routes are used for USGS leveling applications because most surveys are perpetuated from a single fiducial point and are initiated to bring the datum to project sites.

An open traverse (OT) originates at a point of known location and terminates at a point of unknown location (fig. 22). Unlike a closed traverse, an OT is considered mathematically open because closeout to the fiducial mark at the origin point, or other trusted survey marker, is not required (Brinker and Minnick, 1995). Measurement blunders and the accumulation of error are difficult to detect when running an OT (USACE, 2007; Ogundare, 2016), so it is critical to use additional quality-assurance procedures, which are described in the subsequent "Open Traverse" section.

Methods to establish a point of origin, datum, and baseline for trigonometric-leveling surveys are identical for a SRLT, DRST, and OT. A modified version of leap-frog leveling (previously described) is the surveying method used for traverse surveys. Minor adjustments to the workflow of the modified leap-frog leveling method are specific to each traverse approach, which is detailed in subsequent sections.

\section{Single-Run Loop Traverse}

A SRLT begins on a fiducial survey marker at the origin and terminates on the same mark (fig. 22). Leveling measurements are made in the forward direction from the origin point to the destination and then from the destination to the origin point along a different leveling route (Schomaker and Berry, 1981). Reciprocal observations are made in F1 and F2 for each leveling leg, or section, of the traverse and are used to determine section misclosure and to adjust and refine field measurements. The final measurement of the traverse is a closeout observation to the fiducial survey marker at the origin point, and a reciprocal observation from the origin point to evaluate the misclosure of the final section. LCE is determined and is used as a check for measurement blunders and indicates the accumulation of systematic error in the traverse. The leveling route of a SRLT resembles a loop or a series of successive leveling sections (legs) that are connected to form a polygon. A loop traverse is used to perpetuate the datum to sites that are spatially distributed in a nonlinear geometry. For example, if multiple cross sections were required along a meandering reach of river for a hydraulic model, a loop traverse may be considered, especially if the distance to the origin point from the final cross section is shorter than the distance required to retrace the leveling route back to origin. The origin point is the only control point in a loop traverse that is occupied twice (redundant occupation) because leveling only occurs in the forward direction. The procedure for running a SRLT is described below and illustrated in figure 23. The survey method is a modified version of what is commonly referred to as "leap-frog" or "forced-centering" leveling. For this hypothetical SRLT, a terrestrial approach (see the "Establishing a Datum" section) was used to perpetuate the datum from 
an NGS vertical order 2 benchmark to the origin point. The NAVD 88 elevation of the origin point is $1,000.64 \mathrm{ft}$. The horizontal coordinate of the origin point is assumed to be $5,000 \mathrm{ft}$ north and 5,000 ft east. The baseline azimuth from the origin point to the foresight is $90^{\circ} 00^{\prime} 00^{\prime \prime}$, which was determined by a compass measurement; the baseline length $(411.223 \mathrm{ft})$ and the mark-to-mark VD between the baseline points $(0.226 \mathrm{ft})$ were both determined by the total station.

The SRLT leveling procedure is as follows:

1. Level the tripod and prism assembly over the backsight survey marker (point number 1 in fig. 23A). Set a foresight survey marker (point number 3 ) and level the tripod and prism assembly over it. Measure and record the SHs in a field book or standardized field form (fig. 23B; app. 1).

2. Set up the total station above the survey marker (point number 2) between the foresight and backsight markers. Ensure the line of sight is unobstructed to the foresight and to the backsight. Measure and record the $\mathrm{HI}$ in a field book or standardized field form (fig. 23B; app. 1).

3. Start a "new" job in the data-collection device. Enter the XYZ coordinates of the backsight (point 1) and total station (point 2) - these points define the baseline. The horizontal datum can be assumed or referenced to a local or geodetic datum, the vertical datum is the datum of the origin point for the survey. For this hypothetical example, the horizontal datum is assumed, and the vertical datum is NAVD 88 . The coordinates for both points should be known from the procedure to establish a baseline. Record the backsight, total station, and foresight points in a field book or standardized field form (fig. 23B; app. 1).

4. Enter meteorological measurements and ensure the datacollection device is applying corrections for curvature and refraction. Ensure the prism(s) offset is entered into the data-collection device and a correction is being applied. Record the meteorological measurements and the prism(s) offset in a field book or standardized field form (fig. 23B; app. 1).

5. If the data-collection device has a program to account for measurement uncertainty from axial defects, enter the corrections that were determined during preparation procedures for vertical and horizontal collimation error and the tilting-axis error.

6. Enter instrument heights for the backsight (point 1), station (point 2), and the foresight (point 3). Verify the station setup with a backsight check.

7. Program the data-collection device to measure in F1 and F2 and configure the display to show the VD and horizontal distance.
8. Take an F1 observation on the backsight and store the measurement in the data collector. Record the VD and horizontal distance in a field book or standardized form.

9. In F1, point the total station at the foresight and take a measurement. Store the measurement and record the VD and horizontal distance in a field book or standardized form.

10. Invert the scope and point the total station to the foresight again. Take an F2 measurement and store the observation. Record the VD and horizontal distance.

11. In F2, point the total station to the backsight and take a measurement. Record the VD and horizontal distance. After storing the final measurement, "setup 1" is complete if the section misclosure (leveling line between points 1 and 2) is within the maximum allowable error threshold (fig. 23B). Ensure angle and distance thresholds have not been exceeded; these thresholds can be set in the data collector to warn the operator if the difference between the F1 and F2 observation (for angles and distances) exceeds the indicated limit. If the data collector indicates a threshold has been exceeded, another set of $\mathrm{F} 1$ and $\mathrm{F} 2$ measurements may be required.

12. Swap the total station (survey marker 2) and the prism (survey marker 3) for "setup 1," so that the prism is now at survey marker 2 for "setup 2," and the total station is at survey marker 3 for "setup 2" (fig. 23A). The leap-frog method requires interchangeable equipment so simply unlock the tribrach, remove the surveying instrument (total station or prism), and carry it in its protective casing to the indicated location.

13. The tripod and prism assembly that was set up above the backsight (survey marker 1) for "setup 1" should be moved up, centered, and leveled over (set) survey marker 4, which is the foresight for "setup 2" (fig. 23A).

14. For "setup 2", the backsight, total station, and foresight point numbers are 2,3 , and 4 , respectively (fig. 23A). Measure and enter the instrument heights into the datacollection device. Verify the station setup in the datacollection device with a backsight check.

15. Once the total station and backsight have been verified, program the instrument to measure in F1 and F2. Repeat steps 8 through 11, then move the surveying instruments to "setup 3" configuration (fig. 23A).

16. Repeat steps 12 through 15 by incrementally moving up the backsight, station, and foresight from "setup 3" to "setup 4" configuration. Store the foresight (origin) as point number 100 to ensure the data collector does not overwrite point 1 . Inverse point 1 to point 100 for a quick check on LCE. 
$\boldsymbol{A}$

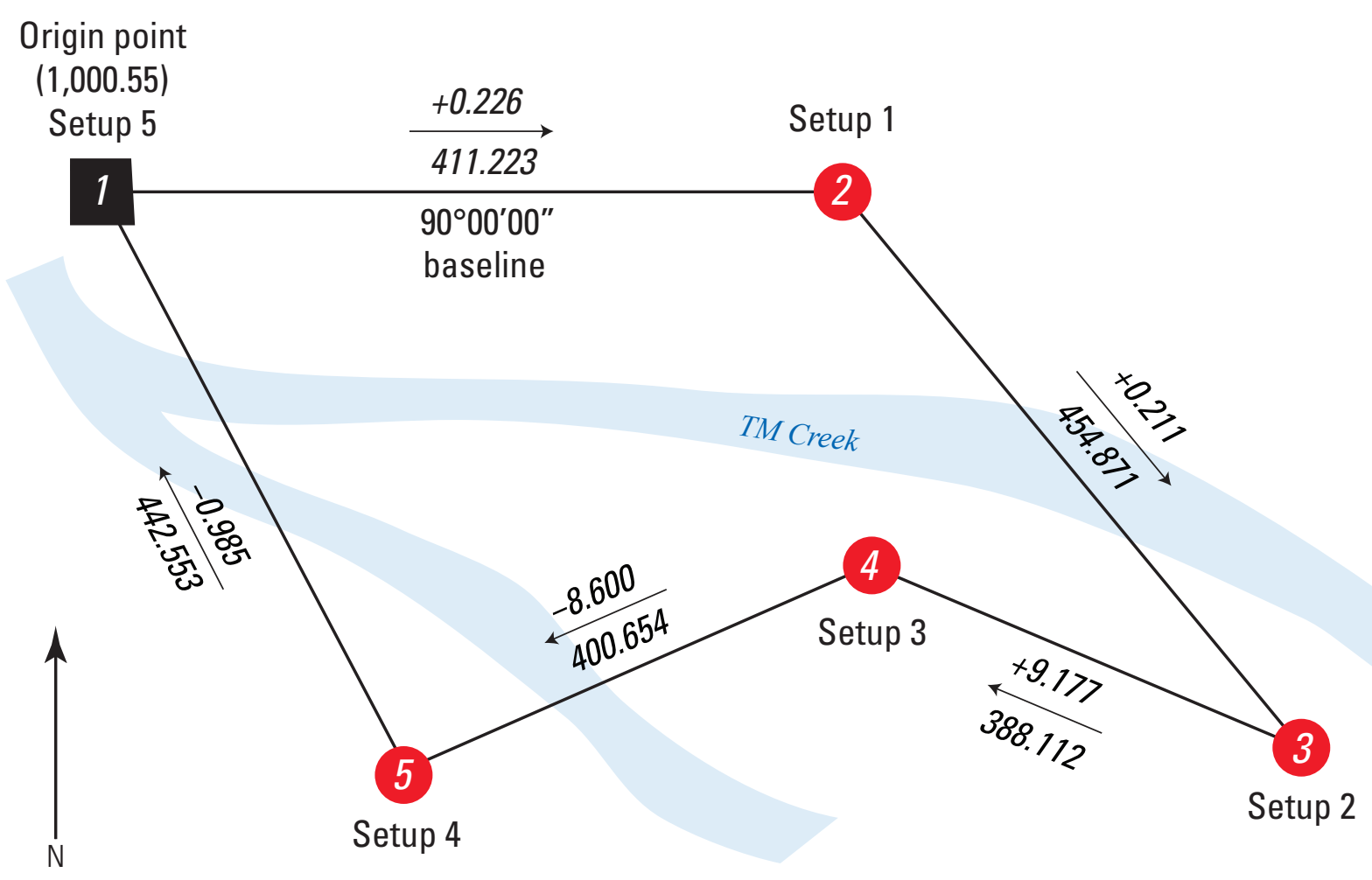

NOT TO SCALE

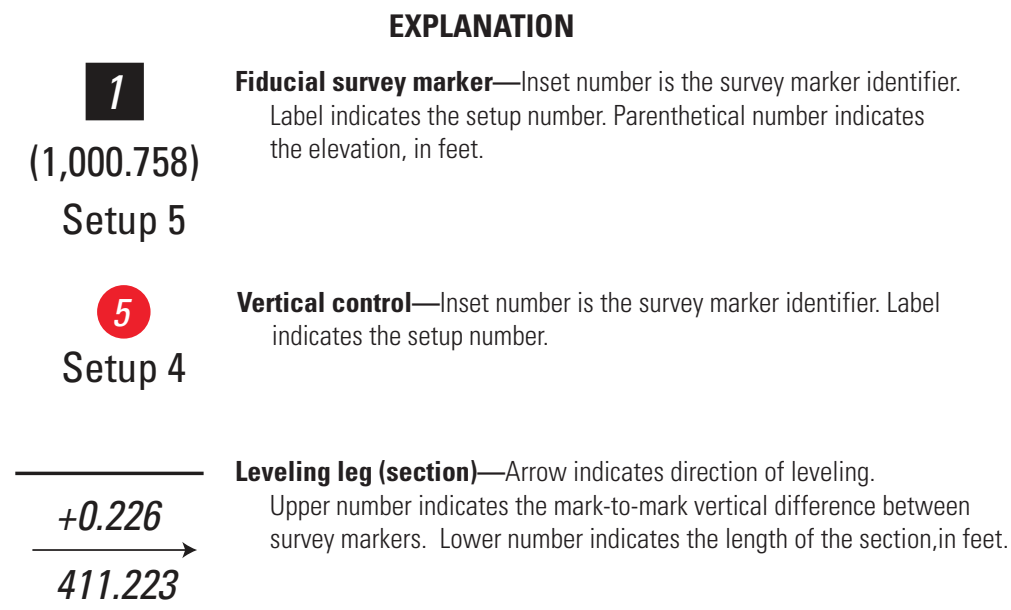

Figure 23. Hypothetical single-run loop traverse. $A$, diagram of levels circuit showing mark-to-mark vertical differences and section lengths; $B$, populated field form; and $C$, modified leap-frog surveying method. Shows arrangement of the trigonometric leveling equipment as the survey progresses from setup 1 to setup 5 . 
$\boldsymbol{B}$

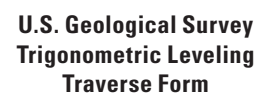

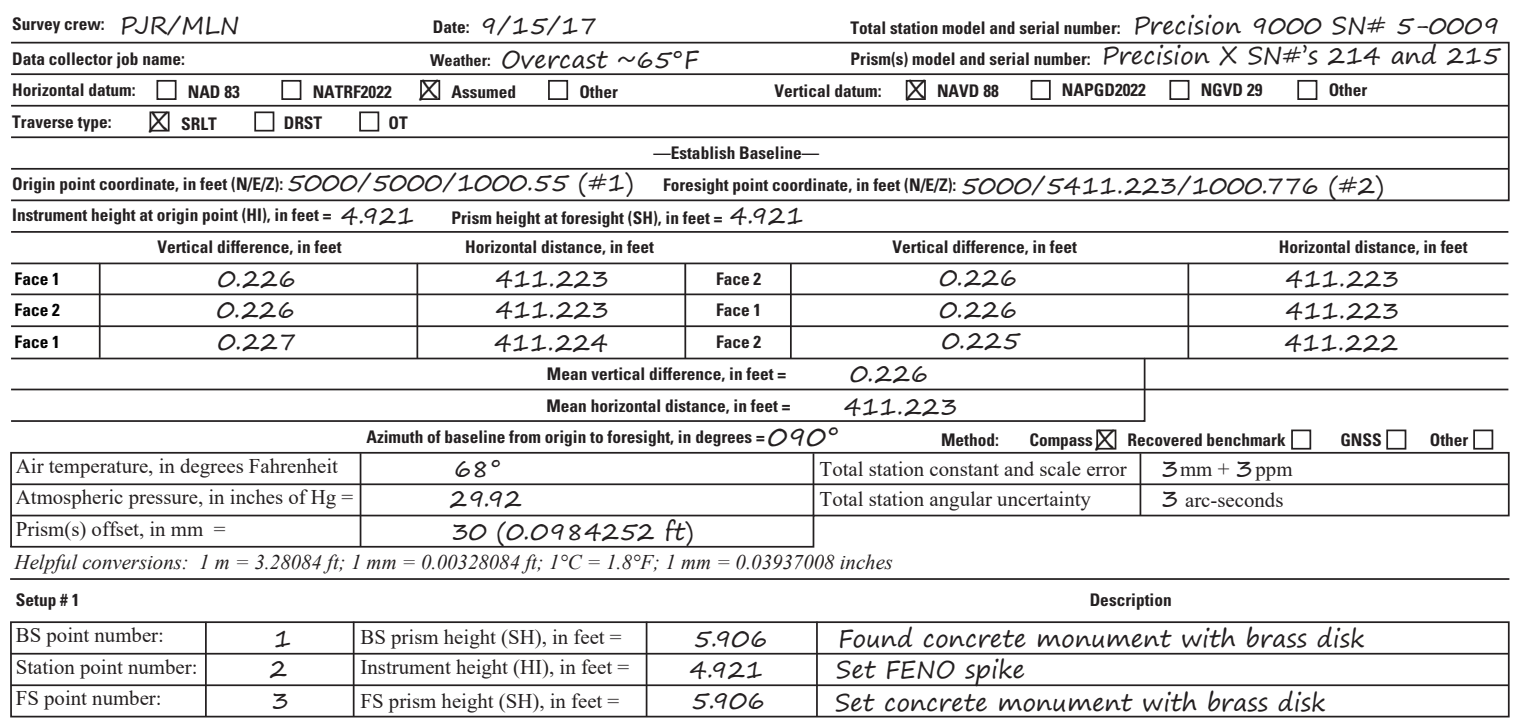

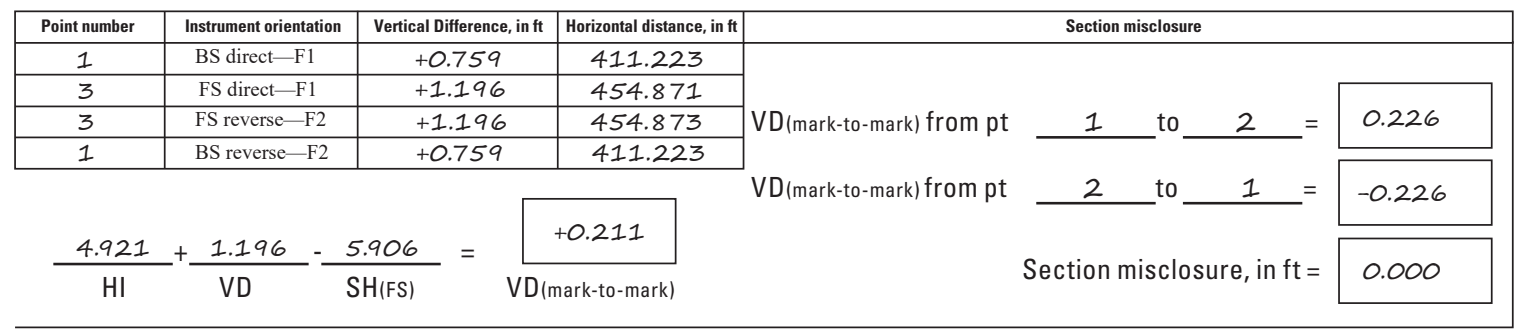

Setup \# 2

BS point number:

Station point number:

\begin{tabular}{|l|l|l|}
\hline FS point number: & 4 & FS prism height $(\mathrm{SH})$, in feet $=$ \\
\hline
\end{tabular}

\begin{tabular}{|l|l|}
\hline \multicolumn{1}{|c|}{ Description } \\
\hline 4.921 & Survey marker \#2 \\
\hline 4.921 & Survey marker \#3 \\
\hline 6.562 & Survey marker \#4; set concrete monument with brass disk \\
\hline
\end{tabular}

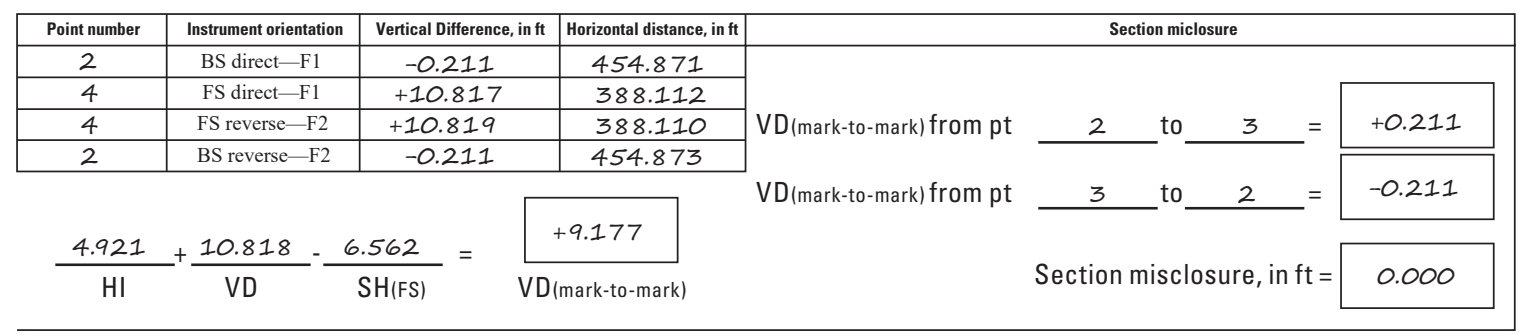

Setup \# 3

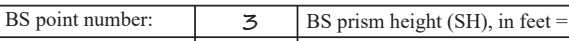

Station point number:

\begin{tabular}{|c|l}
\hline 4 & Instrument height $(\mathrm{HI})$, in feet $=$ \\
\hline 5 & FS prism height $(\mathrm{SH})$, in feet $=$
\end{tabular}

\begin{tabular}{|c|}
\hline 4.921 \\
\hline 4.921 \\
\hline 6.562 \\
\hline
\end{tabular}

Description

FS point number:

\begin{tabular}{|c|c|c|c|}
\hline Point number & Instrument orientation & Vertical Difference, in $\mathrm{ft}$ & Horizontal distance, in $\mathrm{ft}$ \\
\hline 3 & BS direct-F1 & -9.177 & 388.112 \\
\hline 5 & FS direct-F1 & -6.959 & 400.654 \\
\hline 5 & FS reverse-F2 & -6.958 & 400.655 \\
\hline 3 & BS reverse-F2 & -9.177 & 388.110 \\
\hline
\end{tabular}

Survey marker \#4

Survey marker \#5; set FENO spike

$$
\frac{4.921}{H I}+\frac{-6.959}{V D}-\frac{6.562}{S H(F S)}=\frac{-8.600}{V D \text { (mark-to-mark) }}
$$

\begin{tabular}{|c|c|c|c|c|}
\hline \multicolumn{5}{|c|}{ Section misclosure } \\
\hline$V D$ (mark-to-mark) from pt & 3 & to & $=$ & +9.177 \\
\hline $\mathrm{VD}$ (mark-to-mark) from pt & 4 & to & 3 & -9.177 \\
\hline \multicolumn{4}{|c|}{ Section misclosure, in $\mathrm{ft}=$} & 0.000 \\
\hline
\end{tabular}

Figure 23. Hypothetical single-run loop traverse. $A$, diagram of levels circuit showing mark-to-mark vertical differences and section lengths; $B$, populated field form; and $C$, modified leap-frog surveying method. Shows arrangement of the trigonometric leveling equipment as the survey progresses from setup 1 to setup 5 . 


\section{Survey Crew: PJR/MLN}

Job Name: Hypothetical single-run loop traverse
U.S. Geological Survey

Trigonometric Leveling

Traverse Form

Date: $9 / 15 / 17$

Setup \# 4

BS point number:

Station point number:

FS point number:

\begin{tabular}{|c|}
4 \\
5 \\
100
\end{tabular}

\begin{tabular}{|l|l|}
\hline BS prism height $(\mathrm{SH})$, in feet $=$ & \\
\hline Instrument height $(\mathrm{HI})$, in feet $=$ & \\
\hline FS prism height $(\mathrm{SH})$, in feet $=$ &
\end{tabular}

4.921
4.921
4.921

Description

\begin{tabular}{|c|c|c|c|}
\hline Point number & Instrument orientation & Vertical Difference, in ft & Horizontal distance, in ft \\
\hline 3 & BS direct-F1 & 8.603 & 400.654 \\
\hline 100 & FS direct-F1 & -0.985 & 442.552 \\
\hline 100 & FS reverse-F2 & -0.985 & 442.554 \\
\hline 3 & BS reverse-F2 & 8.601 & 400.655 \\
\hline
\end{tabular}

$$
\frac{4.921}{\mathrm{HI}}+\frac{-0.985}{\mathrm{VD}}-\frac{4.921}{\mathrm{SH}(\mathrm{FS})}=\frac{-0.985}{\mathrm{VD} \text { (mark-to-mark) }}
$$
Survey marker \#4 Survey marker \#5 Survey marker \#1; closeout observation

\section{Setup \# 5}

BS point number: Station point number:

FS point number:

\begin{tabular}{l|l|}
\hline 5 & \\
100 & \\
101 & \\
\hline
\end{tabular}

\begin{tabular}{|l|l|l|}
\hline BS prism height $(\mathrm{SH})$, in feet $=$ & 4.921 & \\
\hline Instrument height $(\mathrm{HI})$, in feet $=$ & 4.921 & \\
\hline FS prism height $(\mathrm{SH})$, in feet $=$ & 4.921 & \\
\hline
\end{tabular}

VD (mark-to-mark) from $p$

Section misclosure

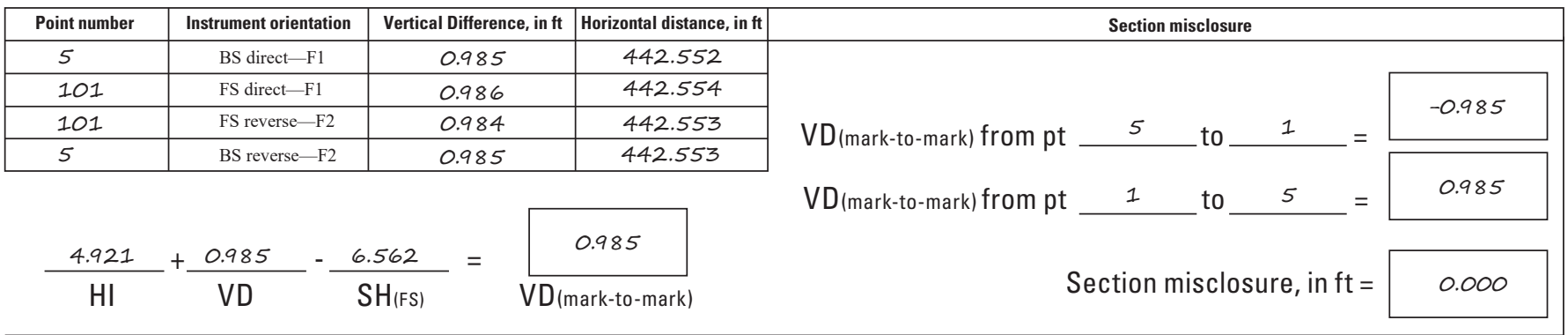

Figure 23. Hypothetical single-run loop traverse. $A$, diagram of levels circuit showing mark-to-mark vertical differences and section lengths; $B$, populated field form; and $C$, modified leap-frog surveying method. Shows arrangement of the trigonometric leveling equipment as the survey progresses from setup 1 to setup 5 . 
C
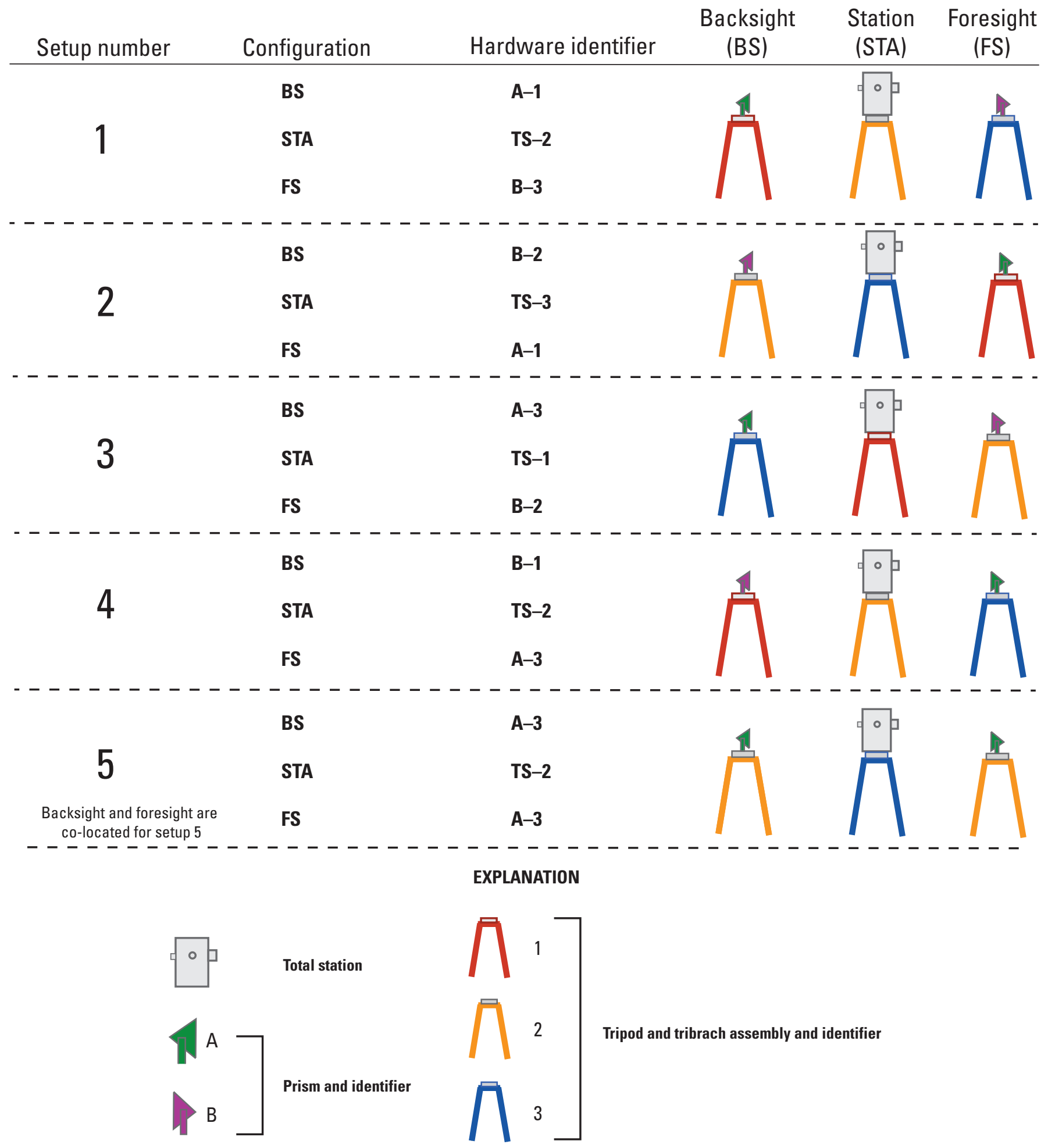

Figure 23. Hypothetical single-run loop traverse. $A$, diagram of levels circuit showing mark-to-mark vertical differences and section lengths; $B$, populated field form; and $C$, modified leap-frog surveying method. Shows arrangement of the trigonometric leveling equipment as the survey progresses from setup 1 to setup 5 . 
17. "Setup 5" is the last setup of the traverse. The purpose of this setup is to collect a reciprocal observation for the last section of the loop, which is defined by the origin survey marker and survey marker 5 . The backsight and foresight are co-located at survey marker 5 , and the station is at the origin survey marker. The backsight, station, and foresight numbers are 5, 100, and 101, respectively. The foresight is stored as point 101 to ensure the data collector does not overwrite point 5 . Because the backsight and foresight are co-located, repointing between observations may be unnecessary but should be verified by the operator. This final measurement can also be made using a unidirectional approach (similar to the baseline procedure), which does not require a foresight observation in the measurement sequence.

The circuit is considered closed after F1 and F2 measurements have been made at "setup 5" and point number 101 is stored. The inverse tool in the coordinate geometry program can be used to ensure the traverse is closed and no major blunders occurred by comparing the coordinates (XYZ) of point numbers 1 and 100. LCE, which is detailed in the subsequent "Adjustments of field measurements" section, may indicate measurement blunders and accumulation of systematic errors in the traverse.

\section{Double-Run Spur Traverse}

A DRST begins on a fiducial survey marker at the origin and terminates on the same mark (fig. 22). Measurements are made in the forward direction from the origin to the destination and are then retraced along the same leveling route in the backward direction from the destination to the origin. A spur is defined as a line segment (also called a section) or a series of successive line segments that branch off the main leveling line and are not part of the existing vertical-control network (Schomaker and Berry, 1981). When the spur is leveled from the origin to the destination, it is considered forward running, and when it is leveled from the destination to the origin, it is considered backward running - the two runnings of the spur are collectively defined as a "double run" traverse and should be completed during different environmental conditions. Suggested practice is to level in the forward direction in the morning and the backward direction in the afternoon (Schomaker and Berry, 1981). In addition to the nearsimultaneous reciprocal observations, the DRST also requires that nonsimultaneous reciprocal observations (reciprocal measurements that are offset by a substantial period of time, also called double-run observations) are made for each segment of the spur - the first observation is made during the forward running and the second observation is made in the reciprocal direction during the backward running. Every control point in a DRST is occupied twice by the total station - the first occupation occurs as the traverse progresses in the forward direction along the spur from the origin point to the destination; and the second occupation occurs in the backward direction along the spur from the destination back to the origin point of the traverse (figs. 22 and 24).

The procedure for running a DRST and SRLT to the destination are identical (steps 1 through 14 in the following procedure); however unlike a SRLT that returns to the origin along a new leveling route, the DRST returns to the origin along the same route (fig. 24). For this hypothetical DRST, a GNSS was used to establish the datum and to determine the azimuth of baseline $\left(179^{\circ} 10^{\prime} 15^{\prime \prime}\right)$ - the NAVD 88 elevation of the origin point is $20.50 \mathrm{ft}$; the State Plane coordinate referenced to the North American Datum of 1983 (NAD 83) is $250,125.15 \mathrm{ft}$ north and $288,001.51 \mathrm{ft}$ east. The baseline length is $325.413 \mathrm{ft}$ and the mark-to-mark VD between the baseline points is $-0.418 \mathrm{ft}$, which was determined by the total station using the previously described procedure to establish a baseline.

The DRST leveling procedure is as follows:

1. Level the tripod and prism assembly over the backsight survey marker (point number 1 in fig. 24). Set a foresight survey marker (point number 3 ) and level the tripod and prism assembly over it. Measure and record the SHs in a field book or standardized field form (app. 1).

2. Set up the total station above the survey marker (point number 2) between the foresight and backsight markers. Ensure the line of sight is unobstructed to the foresight and to the backsight. Measure and record the $\mathrm{HI}$ in a field book or standardized field form (app. 1).

3. Start a "new" job in the data-collection device. Enter the XYZ coordinates of the backsight (point 1) and total station (point 2) - these points define the baseline. The horizontal datum for this hypothetical example is NAD 83 and the vertical datum is NAVD 88 . The coordinates for both points should be known from the procedure to establish a baseline. Record the backsight, total station, and foresight points in a field book or standardized field form.

4. Enter meteorological measurements and ensure the datacollection device is applying corrections for curvature and refraction. Ensure the prism(s) offset is entered into the data-collection device and a correction is being applied. Record the meteorological measurements and the prism(s) offset in a field book or standardized field form.

5. If the data-collection device has a program to account for measurement uncertainty from axial defects, enter the corrections that were determined during preparation procedures for vertical and horizontal collimation error and the tilting-axis error.

6. Enter instrument heights for the backsight (point 1), station (point 2), and the foresight (point 3). Verify the station setup with a backsight check. 

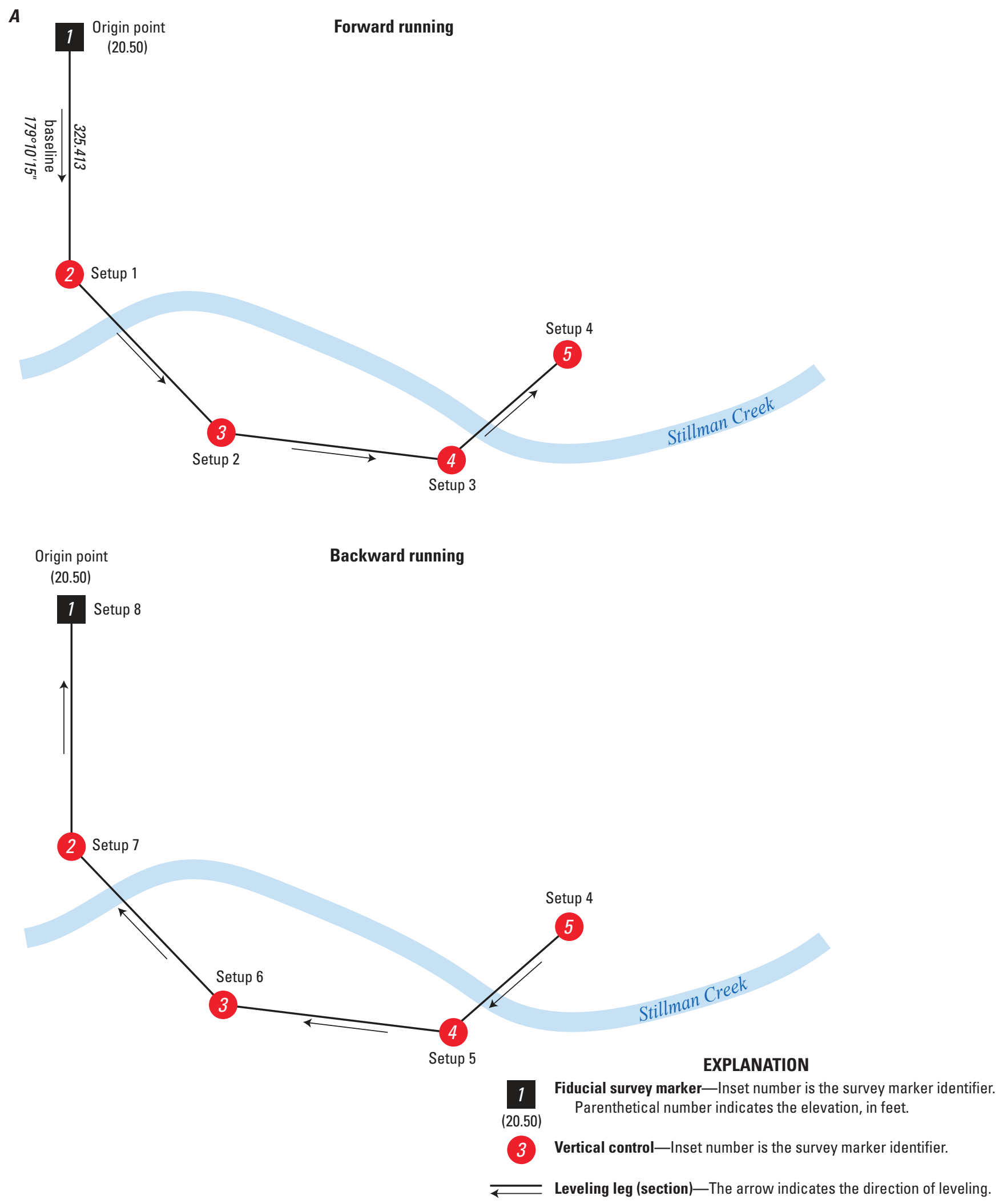

Figure 24. Hypothetical double-run spur traverse. 
7. Program the data-collection device to measure in F1 and F2 and configure the display to show the VD and horizontal distance.

8. Take an F1 observation on the backsight and store the measurement in data-collection device. Record the VD and horizontal distance in a field book or standardized form.

9. In F1, point the total station at the foresight and take a measurement. Store the measurement and record the VD and horizontal distance in a field book or standardized form.

10. Invert the scope and point the total station to the foresight again. Take an F2 measurement and store the observation. Record the VD and horizontal distance.

11. In F2, point the total station to the backsight and take a measurement. Record the VD and horizontal distance. After storing the final measurement, "setup 1" is complete if angle and distance thresholds have not been exceeded. Angle and distance thresholds can be set in the data collector to warn the operator if the difference between the F1 and F2 observation (for angles and distances) exceeds the indicated limit. If the data collector indicates a threshold has been exceeded, another set of F1 and F2 measurements may be required.

12. Swap the total station (survey marker 2) and the prism (survey marker 3) for "setup 1" so that the prism is now at survey marker 2 for "setup 2" and the total station is at survey marker 3 for "setup 2" (fig. 24). The leap-frog method requires interchangeable equipment so simply unlock the tribrach, remove the surveying instrument (total station or prism), and carry it in its protective casing to the indicated location.

13. The tripod and prism assembly that was set up above the backsight (survey marker 1) for "setup 1" should be moved up, centered, and leveled over (set) survey marker 4, which is the foresight for "setup 2" (fig. 24).

14. For "setup 2," the backsight, total station, and foresight point numbers are 2, 3, and 4, respectively (fig. 24). Measure, then enter the instrument heights into the datacollection device. Verify the station setup in the datacollection device with a backsight check.

15. Once the total station and backsight have been verified, program the instrument to measure in F1 and F2. Repeat steps 8 through 11, and then move the surveying instruments to "setup 3."

16. For setup 3, the backsight, total station, and foresight point numbers are 3, 4, and 5, respectively (fig. 24). Enter the instrument heights and verify the station setup with a backsight check. Configure the data collector to measure in F1 and F2. Repeat steps 8 through 11, and then move the surveying instruments to "setup 4." The unique configuration for setup 4 is detailed in step 17.

17. For setup 4, swap the total station (survey marker 4) and the prism (survey marker 5). The prism and tripod at survey marker 3 should not be moved and will not be measured during setup 4 . The backsight is at survey marker 4 , the station is at survey marker 5 , and the foresight will also be at survey marker 4 . In other words, the backsight and foresight are co-located at survey marker 4.

18. Once the station setup has been verified with a backsight check, program the instrument to measure in F1 and F2. Repeat steps 8 through 11, but keep in mind the instrument may not have to be repointed during the F1 and F2 measurements (but should be checked) because the backsight and foresight are co-located at survey marker 4. Once collected, the foresight measurement of survey marker 4 should be identified in the data collector with a "-B" extension (for example, point number "4-B") to indicate the second observation of the survey marker was made during the backward running of the spur traverse (fig. 24). Point 4-B should be stored as an independent measurement in the data collector so it is does not overwrite a previously stored point.

19. The forward run is complete after F1 and F2 measurement have been collected at the backsight for setup 4 . The backward running begins when F1 and F2 measurements have been collected at the foresight for setup 4 (subsequently stored as point 4-B).

20. Leap-frog the surveying instruments to "setup 5" configuration - the backsight, station, and foresight point numbers are 5, 4-B, and 3-B, respectively. Verify the station setup with a backsight check, and then program the data-collection device to measure in F1 and F2. Repeat steps 8 through 11 .

21. Repeat step 20 for setup 6-the backsight, station, and foresight numbers for setup 6 are 4-B, 3-B, and 2-B, respectively.

22. Repeat step 20 for setup 7-the backsight, station, and foresight numbers for setup 7 are $3-\mathrm{B}, 2-\mathrm{B}$, and $1-\mathrm{B}$, respectively.

23. Leap-frog the surveying instruments to "setup 8" configuration. This is a unique configuration because a foresight will not be observed during the measurement routine - the backsight and station point numbers are 2-B and 1-B, respectively. This is the final setup for the spur traverse. Observe F1 and F2 measurements from the station to the backsight. This can be done using a unidirectional approach like the baseline procedure. The purpose of this setup is to get a reciprocal measurement 
for the last section of the spur. Record the VD between the station and the backsight in a standard field form or fieldbook.

The survey chief can choose a noncontinuous DRST, which means some time elapses between the forward and backward running of the spur; step 17 will have to be repeated to ensure reciprocal observations of the first section of the backward running. A noncontinuous traverse ensures environmental conditions have changed between the forward and backward running, which may increase the quality of the final elevations. However, the survey chief needs to consider the quality and stability of the established survey markers, logistics, and time and budget constraints if they choose to complete the backward running on another day. If movement of a survey marker is suspected, it should be documented in the field notes with the understanding that the section misclosure may exceed the specification for the desired standard (described in subsequent sections). Best practice is to complete the forward running in the morning and the backward running in the afternoon to ensure environmental conditions have changed (Schomaker and Berry, 1981) and another field visit is not required to complete the traverse. Always set surveys markers in a stable location that is not subject to landsurface motion, potential land development, vehicle and heavy foot traffic, vandalism, or other hazards that can potentially damage or destroy the mark.

\section{Open Traverse}

The OT method has been developed to accommodate control surveys for project sites where traversing back to the origin point may be impractical or potentially risk the safety of the survey crew. An OT begins on a fiducial survey marker at the origin and terminates at an unknown mark (fig. 22). Measurement blunders and other sources of error are difficult to detect when running an OT (USACE, 2007; Ogundare, 2016), so it is critical to use appropriate quality-assurance procedures. According to literature sources, double-rodded or double-line leveling is a modified version of conventional differential leveling that requires running two simultaneous level routes (Clayton, 1947; Mish, 2008). Unlike conventional differential levels, double-rodded leveling requires two independent sets of backsight and foresight measurements, which provides a continuous check on measured elevations. A modified version of double-rodded leveling has been adapted in this manual for running OT trigonometric-leveling surveys. Double-rodded trigonometric leveling requires two sets of F1 and F2 observations per station - the SH of the foresight is changed between measurements sets to ensure two independent results (fig. 25; Whalem, 1985). The most efficient way to change the target height between measurement sets is to add a fixed-height pole extension (typically $0.2 \mathrm{~m}$ ) to the prism assembly; this extension preserves leveling and centering over the ground point during the height adjustment. Prism height adjustments at the foresight can also be done by adjusting the legs of the tripod or changing the level of a dowel stop for a tripod equipped with an adjustable center pole. The two independent measurements are evaluated by the surveyor to ensure the difference does not exceed the specifications for the desired standard (described in subsequent sections). If the specification is not exceeded the two measurements are averaged to determine the mark-to-mark VD between the station and target. If the specification is exceeded a third set of measurements may be required. If an erroneous measurement is indicated it should be discarded and not included in the computation of the foresight elevation.

The modified version of leap-frog trigonometric leveling described in this manual is the surveying method for OTs except an additional quality-assurance step, which requires changing the foresight target heights between measurement sets, has been added to the workflow. Section misclosure is determined from reciprocal observations and can be used to adjust field measurements. This method is identical to the method to determine section misclosure for closed traverse and is described in the subsequent section. Methods to establish an origin point, datum, and baseline for OTs are also identical to closed traverses and are described in previous sections of this manual. For this hypothetical OT, a satellitebased approach was used to establish the datum on the origin point and orient the baseline. The NAVD 88 elevation of the origin point is $257.78 \mathrm{ft}$ (fig. 25). The baseline azimuth from the origin point to the foresight is $105^{\circ} 41^{\prime} 30^{\prime \prime}$, which was determined by taking a GNSS measurement on both endpoints of the baseline (fig. 25, points 10 and 11) and using coordinate geometry to calculate the azimuth between the points. The length of the baseline is $155.91 \mathrm{ft}$ was determined by the total station (EDM). The procedure for running an OT is described below and illustrated in figure 25 :

1. Level the tripod and prism assembly over the backsight survey marker (point 10 in fig. 25). Set a foresight survey marker (point 12) and level the tripod and prism assembly over it. Measure and record the SHs in a field book or standardized field form (app. 1).

2. Set up the total station above the survey marker (point 11 in fig. 25) between the foresight and backsight markers. Ensure the line of sight is unobstructed to the foresight and to the backsight. Measure and record the $\mathrm{HI}$ in a field book or standardized field form (app. 1).

3. Start a "new" job in the data-collection device. Enter the XYZ coordinates of the baseline points (fig. 25, points 10 and 11). The horizontal datum for this hypothetical example is NAD 83, and the vertical datum is NAVD 88. The coordinates for both points (10 and 11) should be known from the procedure to establish a baseline. Record the backsight, total station, and foresight points in a field book or standardized field form.

4. Enter meteorological measurements and make sure the data-collection device is applying corrections for curvature and refraction. Ensure the prism(s) offset is entered 
$\boldsymbol{A}$

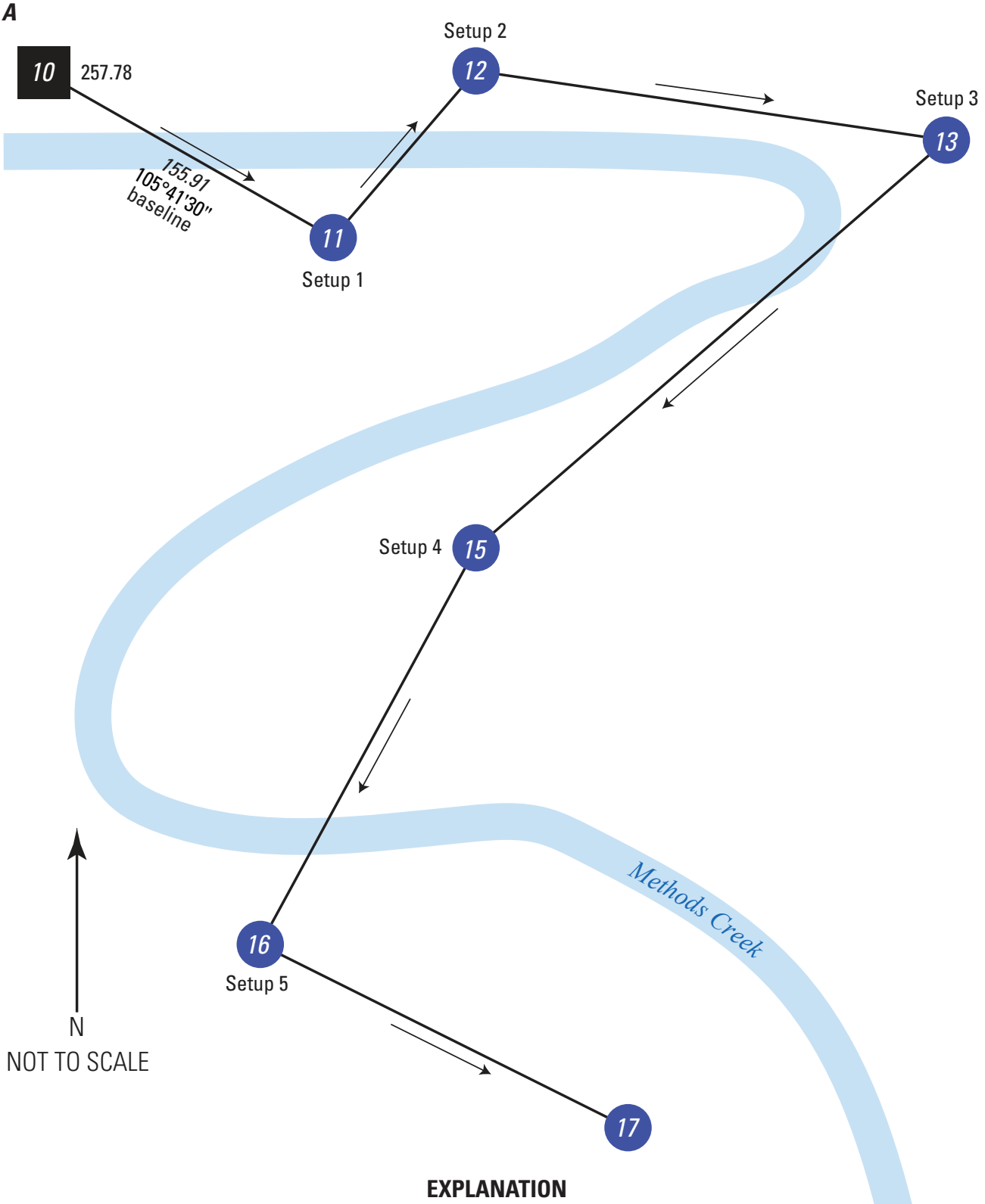

10 Fiducial survey marker-Inset number is the survey marker identifier. Number indicates the known elevation, in feet.

16 Survey marker-Inset number is the survey marker identifier. Leveling leg (section)—Arrow indicates direction of leveling.

Figure 25. Hypothetical open traverse and modified double-rod method. $A$, hypothetical open traverse; and $B$, modified double-rod method. 
B

Measurement 1-Low prism height
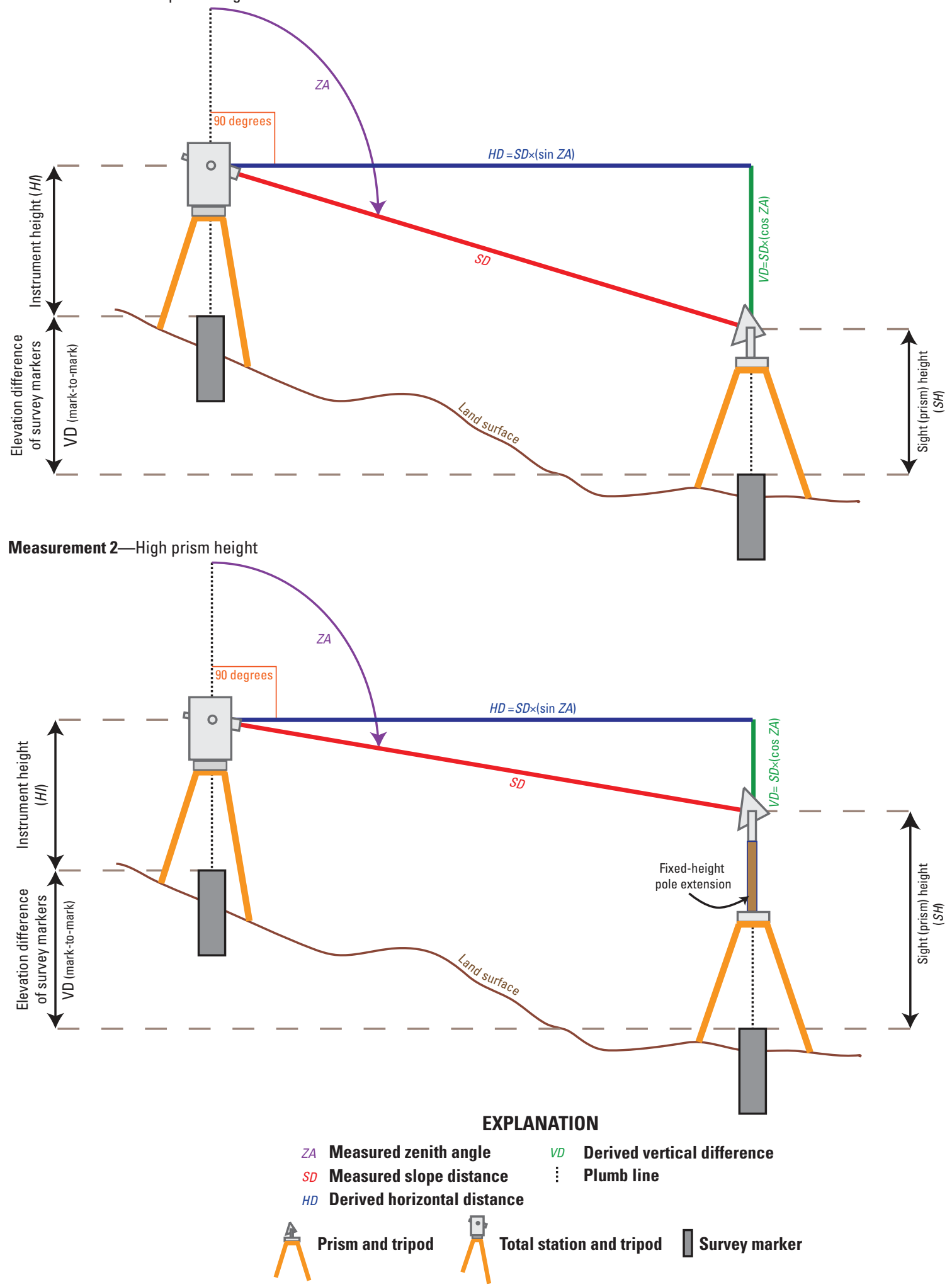

Figure 25. Hypothetical open traverse and modified double-rod method. $A$, hypothetical open traverse; and $B$, modified double-rod method. 
into the data-collection device and a correction is being applied. Record the meteorological measurements and the prism(s) offset in a field book or standardized field form (app. 1).

5. If the data-collection device has a program to account for measurement uncertainty from axial defects, enter the corrections that were determined during preparation procedures for vertical and horizontal collimation error, and tilting-axis error.

6. Enter instrument heights for the backsight (point 10), station (point 11), and the foresight (point 12). Verify the station setup with a backsight check.

7. Program the data-collection device to measure in F1 and F2 and configure the display to show the VD and horizontal distance.

8. Take an F1 observation on the backsight and store the measurement in the data collector. Record the VD and horizontal distance in a field book or standardized form.

9. In F1, point the total station at the foresight, and take a measurement. Store the measurement and record the VD and horizontal distance in a field book or standardized form.

10. Invert the scope and point the total station to the foresight again. Take an F2 measurement and store the observation. Record the VD and horizonal distance.

11. In F2, point the total station to the backsight, and take a measurement. Record the VD and horizontal distance and confirm angle and distance thresholds have not been exceeded. Store the foresight measurement as point 12-L. The "-L" extension to the point number indicates the measurement is from the "low" prism height.

12. Change the SH at the foresight. An efficient way to change the $\mathrm{SH}$ is to add a fixed-height pole extension (typically $0.2 \mathrm{~m}$ ) to the prism assembly.

13. Repeat steps 8 through 11 , and store the measurement as point $12-\mathrm{H}$. The "-H" extension indicates the measurement is from the "high" prism height. Confirm the measurements (mark-to-mark VDs) for point $12-\mathrm{L}$ and $12-\mathrm{H}$ are within third-order specifications $(|\leq 0.015| \mathrm{ft})$.

14. Leap-frog the instrumentation to setup 2 configuration - the backsight, station, and foresight numbers are 11,12 , and 13 , respectively. Verify the station setup with a backsight check and configure the data collector to measure in F1 and F2. Repeat steps 8 through 13. Confirm the measurements for point $13-\mathrm{L}$ and $13-\mathrm{H}$ are within third-order specifications $(|\leq 0.015| \mathrm{ft})$.

\section{Repeat step 14 for setups 3,4 , and 5 .}

Procedures to establish an origin point, datum, and baseline for trigonometric-leveling surveys are identical for a SRLT, DRST, and OT (table 5). A modified version of leapfrog leveling (previously described) is the surveying method for the traverse surveys; however, a DRST requires that all control points are redundantly occupied, and OT requires additional quality assurance, which is a modified version of "double-rodded" leveling (table 6). All traverse types require a fiducial survey marker at the origin point; however, only closed traverse (SRLT and DRST) require returning to that fiducial survey marker as part of the workflow. F1 and F2 reciprocal observations are observed for each traverse type, and section misclosure is evaluated before the instrumentation

Table 6. Summary of criteria for closed and open traverses.

\begin{tabular}{lccc}
\hline \multicolumn{1}{c}{ Traverse type } & $\begin{array}{c}\text { Single-run loop } \\
\text { traverse }\end{array}$ & $\begin{array}{c}\text { Double-run spur } \\
\text { traverse }\end{array}$ & Open traverse \\
\hline Minimum number of fiducial points & 1 & 1 & 1 \\
Reciprocal observations & Yes & Yes & Yes \\
Determine section misclosure (from reciprocal field observations) & Yes & Yes & Yes \\
Determine section misclosure (from double-run observations) & No & Yes & No \\
Modified double-rod method & No & No & Yes \\
Closeout to origin point & Yes & Yes & No \\
Redundant occupation of control points & No & Yes & No \\
Determine closure error of traverse & Yes & Yes & No \\
Distribute closure error of traverse & Yes & No & No \\
Classify trigonometric-leveling survey & Yes & Yes & Yes \\
Suitable to establish vertical control & Yes & Yes & Yes \\
\hline
\end{tabular}

${ }^{1}$ Observed during direct and reverse instrument orientation (F1 and F2, respectively) measurement sets.

2 The origin point is the only point that is occupied twice in a single-run loop traverse.

${ }^{3}$ Must satisfy U.S. Geological Survey Trigonometric Level III specifications for open traverse (table 9). 
is advanced to the next setup in case the section must be releveled due to poor closure. The DRST is more rigorous than SRLT or OT because all control points require redundant occupation; the origin point is the only point that is redundantly occupied during a SRLT, and no control points are occupied twice during an OT. Methods to adjust field measurements are variable for each type of traverse and are detailed in the subsequent sections.

\section{Adjustments of Field Measurements}

The procedures described in this manual were deliberately designed to obtain precise field measurements that require minimum adjustment during postprocessing. If measurement blunders did not occur during the traverse, the survey marker elevation obtained in the field and the final elevation obtained during postprocessing should be nearly equivalent. Adjustments to field measurements for section closure error (misclosure) from reciprocal observations are made for open and closed traverse. After the field measurement (mark-to-mark VD) is adjusted for misclosure, it is identified as preliminary. After the preliminary mark-to-mark VD has been reviewed by an analyst, it can be used to determine the final elevation for vertical-control points in an OT. For closed traverse (DRST and SRLT), additional adjustments are necessary to refine the solution and determine a final elevation.

The preliminary mark-to-mark VD is used to calculate a preliminary elevation for the control points in a SRLT. The LCE is calculated (eq. 16) and distributed to the control points in the network based on distance from the origin - these elevations are considered the final elevations. For a DRST, preliminary mark-to-mark VDs are calculated for the forward and backward running of the spur - these values will have opposite signs and will be used for a second adjustment. The second adjustment is made by determining the misclosure of the preliminary mark-to-mark VDs from the forward running and backward running of the DRST and is described in a subsequent section. Three types of adjustments-section misclosure from reciprocal observations, which is done for all traverse types; section misclosure from double-run observations, which is done for DRST; and loop-closure error, which is done for SRLT - are described in the next section.

\section{Determining Section Misclosure from Reciprocal Observations}

Procedures to run traverse surveys were designed so that reciprocal observations are made for each section of the traverse. Reciprocal observations are not made simultaneously; however, the workflow was developed to minimize the amount of time between measurements to reduce the chance that atmospheric conditions may change along the measurement path. Reciprocal observations are used to offset measurement uncertainty due to atmospheric refraction and curvature but, importantly, can be used to determine the leveling quality of each leveling line (section), which is indicated by the misclosure (fig. 26). Misclosure is determined from reciprocal observations by comparing the mark-to-mark VD between directly connected survey markers. Determining the mark-to-mark VD by using the derived coordinates in the data-collection device is not adequate because these values are typically not adjusted (unless programmed) for the reciprocal observations made during the F1 and F2 measurement sets and may result in an erroneous section misclosure. Misclosure not only indicates systematic measurement errors that are inherent in trigonometric-leveling measurements but can also detect measurement blunders. If a measurement blunder is detected, the section should be releveled. Perfect section closure is indicated when the sum of the mark-to-mark VDs from the reciprocal observations is zero. If the section misclosure is greater than zero, the mark-to-mark VD determined from field measurements should be adjusted to determine a preliminary mark-to-mark VD. The procedure to determine mark-to-mark VDs from raw field measurements (F1 and F2 zenith angles and slope distances, HI, and $\mathrm{SH}$ ), calculate misclosure, determine preliminary mark-to-mark VDs, and derive an elevation of a survey marker is as follows:

1. Export the raw data or job file from the data-collection device, which contains the fundamental measurements observed by the total station during the survey (fig. 8). Ensure this file includes the F1/F2 zenith angles and slope distances and the $\mathrm{HI}$ and $\mathrm{SH}$. The horizontal angle measurements do not have to be exported.

2. Tabulate the zenith angles and slope distances of the reciprocal observations for each section.

3. Reduce the F2 zenith angles to the F1 equivalent, and then average the zenith angles using equation 14 , which is $Z A_{m}=(Z A[F 1]+[360-Z A(F 2)]) \div 2$.

4. Average the slope distances.

5. Calculate the VD from the averaged ZA and SD (fig. 8).

6. Calculate the mark-to-mark VD using equation 15:

$$
V D_{\text {mark-to-mark }}=H I+V D-S H
$$

7. Sum the mark-to-mark VD for the reciprocal observations to obtain the section misclosure.

8. Determine if the magnitude of the misclosure is the result of a systematic error or measurement blunder. If the misclosure is from systematic errors, proceed to step 9; if a measurement blunder is detected, consider releveling the section.

9. Multiply the misclosure (determined in step 7) by -1 , then divide that value in half to determine the adjustment value. 
1) Export the raw data or job file from data collection device which contains the fundamental measurements observed by the total station during the survey (fig. 8). Make sure this includes the direct and reverse instrument orientation (F1 and F2, respectively) zenith angles and slope distances, and the instrument (HI) and prism heights (SH).

Station setup

\begin{tabular}{|l|l|l|l|l|l|l|l|l|l|l|l|}
\hline Station & Instrument height & 4.850 & Station type & Station setup & Scale factor & 1.00000000 & Std Error & & ?
\end{tabular}

Orientation

\begin{tabular}{|c|c|c|c|c|c|c|c|c|c|}
\hline Station & & Backsight point & & $\begin{array}{l}\begin{array}{l}\text { F1 Orientation } \\
\text { correction }\end{array} \\
\end{array}$ & $148^{\circ} 00^{\prime} 44^{\prime \prime}$ & \begin{tabular}{|l|}
$\begin{array}{l}\text { F2 Orientation } \\
\text { correction }\end{array}$ \\
\end{tabular} & $?$ & Orient. Std Err & ? \\
\hline $\begin{array}{l}\text { Point (B.S.) } \\
\text { Std Errors } \\
\text { Target height }\end{array}$ & $\begin{array}{r}4 \\
5.040\end{array}$ & $\begin{array}{l}\text { HA } \\
\text { HA } \\
\text { Prism constant }\end{array}$ & $\begin{array}{c}0^{\circ} 00^{\prime} 00^{\prime \prime} \\
0^{\circ} 00^{\prime} 02^{\prime \prime} \\
-35.0 \mathrm{~mm}\end{array}$ & $\begin{array}{l}\text { VA } \\
\text { VA }\end{array}$ & $\begin{array}{c}88^{\circ} 46^{\prime} 54^{\prime \prime} \\
0^{\circ} 00^{\prime} 02^{\prime \prime}\end{array}$ & $\begin{array}{l}\text { SD } \\
\text { SD }\end{array}$ & $\begin{array}{r}155.935 \\
0.010\end{array}$ & Code & HUB-4 \\
\hline Deltas & 4 & Azimuth & $?$ & H.Dist & 0.001 & V.Dist & -0.001 & & \\
\hline $\begin{array}{l}\text { Point } \\
\text { Std Errors } \\
\text { Target height }\end{array}$ & $\begin{array}{r}2 \\
4.830\end{array}$ & \begin{tabular}{|l|}
$\mathrm{HA}$ \\
HA \\
Prism constant
\end{tabular} & $\begin{array}{r}122^{\circ} 56^{\prime} 40^{||} \\
0^{\circ} 00^{\circ} 02^{\prime \prime} \\
-35.0 \mathrm{~mm}\end{array}$ & VA & $\begin{array}{r}90^{\circ} 04^{\prime} 36^{\prime \prime} \\
0^{\circ} 00^{\prime} 02^{\prime \prime}\end{array}$ & \begin{tabular}{|l} 
SD \\
SD
\end{tabular} & \begin{tabular}{r|}
127.531 \\
0.010
\end{tabular} & Code & HUB-2 \\
\hline
\end{tabular}

Orientation

\begin{tabular}{|c|c|c|c|c|c|c|c|c|c|}
\hline Station & & Backsight point & 4 & & $148^{\circ} 00^{\prime} 44^{\prime \prime}$ & \begin{tabular}{|l|}
$\begin{array}{l}\text { F2 Orientation } \\
\text { correction }\end{array}$ \\
\end{tabular} & $148^{\circ} 00^{\prime} 50^{\prime \prime}$ & Orient. Std Err & $?$ \\
\hline $\begin{array}{l}\text { Point (B.S.) } \\
\text { Std Errors } \\
\text { Target height }\end{array}$ & $\begin{array}{r}4 \\
5.040\end{array}$ & $\begin{array}{l}\mathrm{HA} \\
\text { HA } \\
\text { Prism constant }\end{array}$ & $\begin{array}{c}0^{\circ} 00^{\prime} 00^{\prime \prime} \\
0^{\circ} 00^{\prime} 02^{\prime \prime} \\
-35.0 \mathrm{~mm}\end{array}$ & $\begin{array}{l}\text { VA } \\
\text { VA }\end{array}$ & $\begin{array}{r}88^{\circ} 46^{\prime} 56^{\prime \prime} \\
0^{\circ} 00^{\prime} 02^{\prime \prime}\end{array}$ & $\begin{array}{l}\text { SD } \\
\text { SD }\end{array}$ & $\begin{array}{r}155.934 \\
0.010\end{array}$ & Code & HUB-4 \\
\hline $\begin{array}{l}\text { Point } \\
\text { Std Errors } \\
\text { Target height }\end{array}$ & $\begin{array}{r}2 \\
4.830\end{array}$ & \begin{tabular}{|l|} 
HA \\
HA \\
Prism constant
\end{tabular} & $\begin{array}{r}122^{\circ} 56^{\prime} 40^{\prime \prime} \\
0^{\circ} 00^{\circ} 02^{\circ} \\
-35.0 \mathrm{~mm}\end{array}$ & $\begin{array}{l}\text { VA } \\
\text { VA }\end{array}$ & $\begin{array}{r}90^{\circ} 04^{4} 36^{\prime \prime} \\
0^{\circ} 00^{\prime} 02^{\prime \prime}\end{array}$ & $\begin{array}{l}\text { SD } \\
\text { SD }\end{array}$ & $\begin{array}{r}127.533 \\
0.010\end{array}$ & Code & HUB-2 \\
\hline $\begin{array}{l}\text { Point (B.S.) } \\
\text { Std Errors } \\
\text { Target height }\end{array}$ & $\begin{array}{r}4 \\
5.040\end{array}$ & \begin{tabular}{|l|} 
HA \\
HA \\
Prism constant
\end{tabular} & $\begin{array}{r}179^{\circ} 59^{\prime} 54^{\prime \prime} \\
0^{\circ} 00^{\circ} 02^{\prime \prime} \\
-35.0 \mathrm{~mm}\end{array}$ & $\begin{array}{l}\text { VA } \\
\text { VA }\end{array}$ & $\begin{array}{r}271^{\circ} 13^{\prime} 22^{\prime \prime} \\
0^{\circ} 00^{\prime} 02^{\prime \prime}\end{array}$ & $\begin{array}{l}\text { SD } \\
\text { SD }\end{array}$ & $\begin{array}{r}155.934 \\
0.010\end{array}$ & Code & HUB-4 \\
\hline $\begin{array}{l}\text { Point } \\
\text { Std Errors } \\
\text { Target height }\end{array}$ & $\begin{array}{r}2 \\
4.830\end{array}$ & \begin{tabular}{|l|} 
HA \\
HA \\
Prism constant
\end{tabular} & $\begin{array}{r}302^{\circ} 56^{\prime} 36^{\prime \prime} \\
0^{\circ} 00^{\circ} 02^{\prime} \\
-35.0 \mathrm{~mm}\end{array}$ & $\begin{array}{l}\text { VA } \\
\text { VA }\end{array}$ & $\begin{array}{r}269^{\circ} 55^{\prime} 41^{\prime \prime} \\
0^{\circ} 00^{\prime} 02^{\prime \prime}\end{array}$ & $\begin{array}{l}\text { SD } \\
\text { SD }\end{array}$ & $\begin{array}{r}127.530 \\
0.010\end{array}$ & Code & HUB-2 \\
\hline
\end{tabular}

2) Tabulate the zenith angles and slope distances of the reciprocal observations for each section.

3) Reduce the $F 2$ zenith angles to $F 1$ equivalent, then average the zenith angles. Use the following equation: $Z A m=(Z A[F 1]+[360-Z A(F 2)]) \div 2$.

4) Average the slope distances

\begin{tabular}{|c|c|c|c|c|c|}
\hline & \multirow[b]{2}{*}{ Set } & \multirow[b]{2}{*}{ TS Orientation } & \multicolumn{2}{|c|}{ Zenith Angle, in DD MM SS } & \multirow[b]{2}{*}{ Measured slope distance, in $\mathrm{ft}$} \\
\hline 1 to 2 & & & Measured & Reduced & \\
\hline $\mathrm{HI}=4.850$ & \multirow[b]{2}{*}{1} & $\mathrm{~F} 1$ & $90^{\circ} 04^{\prime} 36^{\prime \prime}$ & $90^{\circ} 04^{\prime} 36^{\prime \prime}$ & 127.533 \\
\hline \multirow[t]{5}{*}{$\mathrm{SH}=4.830$} & & F2 & $269^{\circ} 55^{\prime} 41$ & $90^{\circ} 04^{\prime} 19^{\prime \prime}$ & 127.53 \\
\hline & \multirow[b]{2}{*}{2} & F1 & $90^{\circ} 04^{\prime} 36^{\prime \prime}$ & $90^{\circ} 04^{\prime} 36^{\prime \prime}$ & 127.532 \\
\hline & & $\mathrm{F} 2$ & $269^{\circ} 55^{\prime} 41$ & $90^{\circ} 04^{\prime} 19^{\prime \prime}$ & 127.53 \\
\hline & \multirow[b]{2}{*}{3} & F1 & $90^{\circ} 04^{\prime} 35$ & $90^{\circ} 04^{\prime} 35$ & 127.531 \\
\hline & & F2 & $269^{\circ} 55^{\prime} 41^{\prime \prime}$ & $90^{\circ} 04^{\prime} 19^{\prime \prime}$ & 127.531 \\
\hline & & & Average & $90^{\circ} 04^{\prime} 27$ & 127.531 \\
\hline & & & Mark-to-mark VD, in $\mathrm{ft}$ & -0.145 & \\
\hline
\end{tabular}

\begin{tabular}{|c|c|c|c|c|c|}
\hline & \multirow[b]{2}{*}{ Set } & \multirow[b]{2}{*}{ TS Orientation } & \multicolumn{2}{|c|}{ Zenith Angle, in DD MM SS } & \multirow[b]{2}{*}{ Measured slope distance, in $\mathrm{ft}$} \\
\hline & & & Measured & Reduced & \\
\hline 2 to 1 & \multirow[b]{2}{*}{1} & $\mathrm{~F} 1$ & $89^{\circ} 55^{\prime} 40^{\prime \prime}$ & $89^{\circ} 55^{\prime} 40^{\prime \prime}$ & 127.531 \\
\hline $\mathrm{HI}=4.830$ & & $\mathrm{~F} 2$ & $270^{\circ} 04^{\prime} 33^{\prime \prime}$ & $89^{\circ} 55^{\prime} 27^{\prime \prime}$ & 127.533 \\
\hline \multirow[t]{6}{*}{$\mathrm{SH}=4.850$} & \multirow[b]{2}{*}{2} & F1 & $89^{\circ} 55^{\prime} 38^{\prime \prime}$ & $89^{\circ} 55^{\prime} 38^{\prime \prime}$ & 127.533 \\
\hline & & F2 & $270^{\circ} 04^{\prime} 31^{\prime \prime}$ & $89^{\circ} 55^{\prime} 29^{\prime \prime}$ & 127.531 \\
\hline & \multirow[b]{2}{*}{3} & F1 & $89^{\circ} 55^{\prime} 38^{\prime \prime}$ & $89^{\circ} 55^{\prime} 38^{\prime \prime}$ & 127.531 \\
\hline & & $\mathrm{F} 2$ & $270^{\circ} 04^{\prime} 31^{\prime \prime}$ & $89^{\circ} 55^{\prime} 29^{\prime \prime}$ & 127.531 \\
\hline & & & Average & $89^{\circ} 55^{\prime} 34^{\prime \prime}$ & 127.532 \\
\hline & & & Mark-to-mark VD, in ft & 0.144 & \\
\hline
\end{tabular}

Figure 26. Field measurements of a single-run loop traverse and the procedure to calculate section misclosure from reciprocal observations, determine preliminary mark-to-mark vertical differences, and determine preliminary elevations. 


\begin{tabular}{|c|c|c|c|c|c|}
\hline & \multirow[b]{2}{*}{ Set } & \multirow[b]{2}{*}{ TS Orientation } & \multicolumn{2}{|c|}{ Zenith Angle, in DD MM SS } & \multirow[b]{2}{*}{ Measured slope distance, in $\mathrm{f}$} \\
\hline & & & Measured & Reduced & \\
\hline 2 to 3 & \multirow[b]{2}{*}{1} & $\mathrm{~F} 1$ & $89^{\circ} 41^{\prime} 59^{\prime \prime}$ & $89^{\circ} 41^{\prime} 59^{\prime \prime}$ & 331.209 \\
\hline $\mathrm{HI}=4.830$ & & $\mathrm{~F} 2$ & $270^{\circ} 18^{\prime} 14$ & $89^{\circ} 41^{\prime} 46^{\prime \prime}$ & 331.21 \\
\hline \multirow[t]{6}{*}{$\mathrm{SH}=4.390$} & \multirow[b]{2}{*}{2} & F1 & $89^{\circ} 41^{\prime} 59$ & $89^{\circ} 41^{\prime} 59$ & 331.208 \\
\hline & & F2 & $270^{\circ} 18^{\prime} 13^{\prime \prime}$ & $89^{\circ} 41^{\prime} 47^{\prime \prime}$ & 331.21 \\
\hline & \multirow[b]{2}{*}{3} & $\mathrm{~F} 1$ & $89^{\circ} 41^{\prime} 59^{\prime \prime}$ & $89^{\circ} 41^{\prime} 59^{\prime \prime}$ & 331.209 \\
\hline & & F2 & $270^{\circ} 18^{\prime} 14^{\prime \prime}$ & $89^{\circ} 41^{\prime} 46^{\prime \prime}$ & 331.208 \\
\hline & & & Average & $89^{\circ} 41^{\prime} 53^{\prime \prime}$ & 331.209 \\
\hline & & & Mark-to-mark VD, in ft & 2.185 & \\
\hline
\end{tabular}

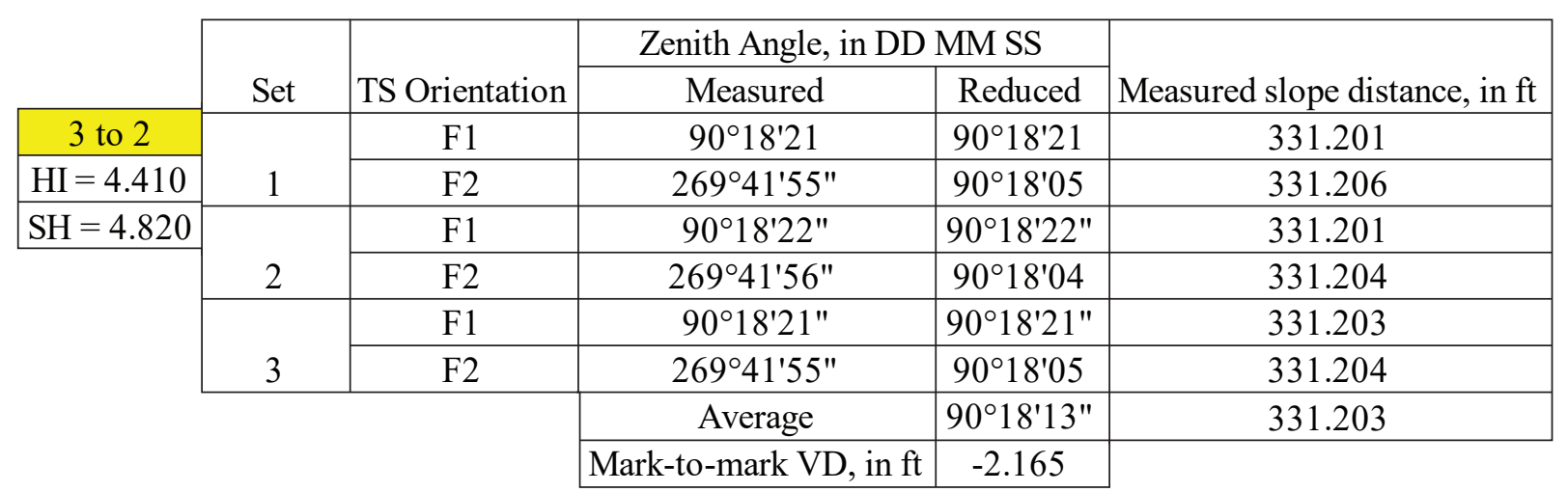

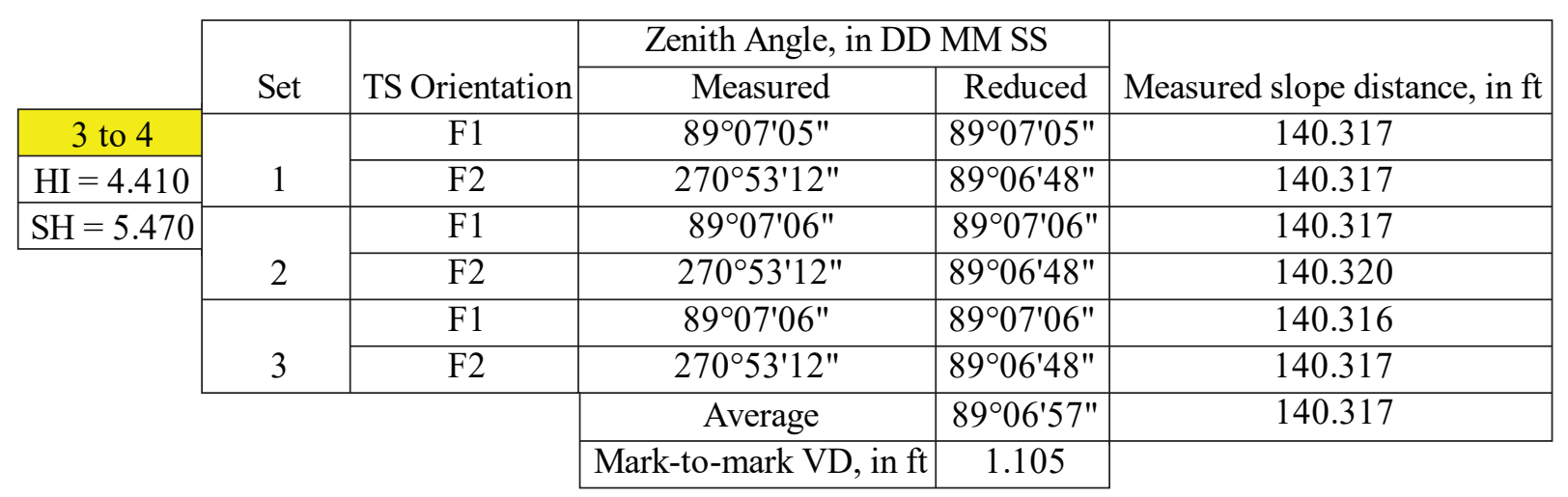

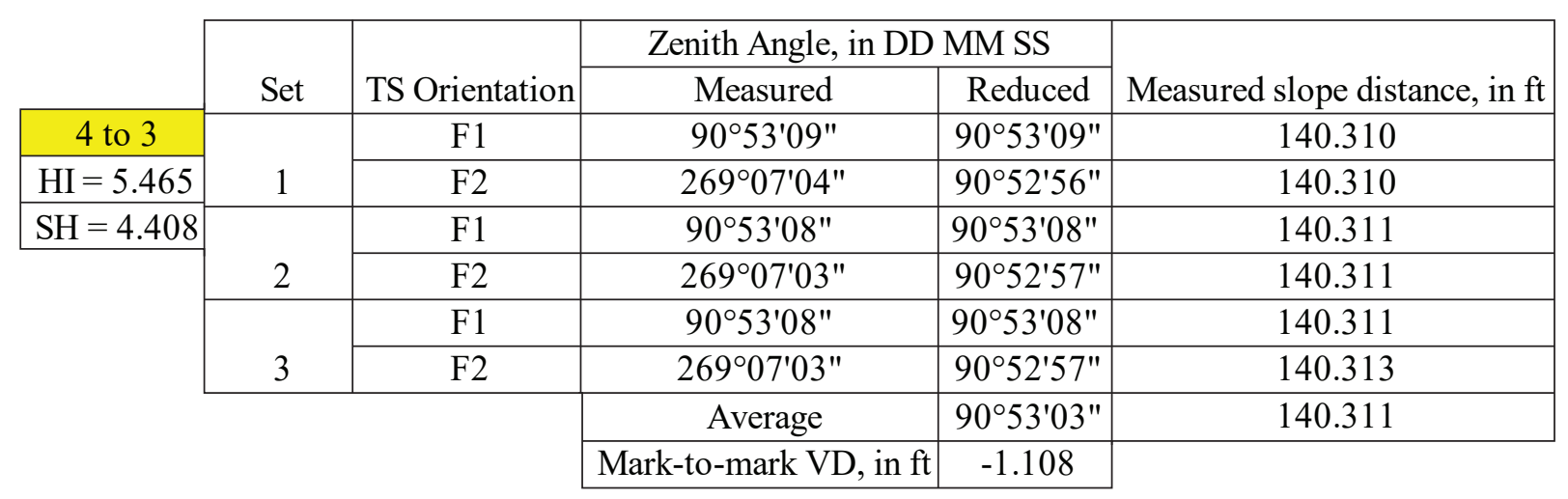

Figure 26. Field measurements of a single-run loop traverse and the procedure to calculate section misclosure from reciprocal observations, determine preliminary mark-to-mark vertical differences, and determine preliminary elevations. 


\begin{tabular}{|c|c|c|c|c|c|}
\hline & \multirow[b]{2}{*}{ Set } & \multirow[b]{2}{*}{ TS Orientation } & \multicolumn{2}{|c|}{ Zenith Angle, in DD MM SS } & \multirow[b]{2}{*}{ Measured slope distance, in $\mathrm{ft}$} \\
\hline & & & Measured & Reduced & \\
\hline 4 to 1 & \multirow[b]{2}{*}{1} & $\mathrm{~F} 1$ & $91^{\circ} 26^{\prime} 46^{\prime \prime}$ & $91^{\circ} 26^{\prime} 46^{\prime \prime}$ & 155.947 \\
\hline $\mathrm{HI}=5.465$ & & F2 & $268^{\circ} 33^{\prime} 26^{\prime \prime}$ & $91^{\circ} 26^{\prime} 34^{\prime \prime}$ & 155.944 \\
\hline \multirow[t]{4}{*}{$\mathrm{SH}=4.670$} & \multirow[b]{2}{*}{2} & F1 & $91^{\circ} 26^{\prime} 46^{\prime \prime}$ & $91^{\circ} 26^{\prime} 46^{\prime \prime}$ & 155.947 \\
\hline & & $\mathrm{F} 2$ & $268^{\circ} 33^{\prime} 26^{\prime \prime}$ & $91^{\circ} 26^{\prime} 34^{\prime \prime}$ & 155.945 \\
\hline & \multirow[b]{2}{*}{3} & F1 & $91^{\circ} 26^{\prime} 47^{\prime \prime}$ & $91^{\circ} 26^{\prime} 47^{\prime \prime}$ & 155.947 \\
\hline & & F2 & $268^{\circ} 33^{\prime} 26^{\prime \prime}$ & $91^{\circ} 26^{\prime} 34^{\prime \prime}$ & 155.945 \\
\hline & & & Average & $91^{\circ} 26^{\prime} 40^{\prime \prime}$ & 155.946 \\
\hline & & & Mark-to-mark VD, in $\mathrm{ft}$ & -3.136 & \\
\hline
\end{tabular}

\begin{tabular}{|c|c|c|c|c|c|}
\hline & \multirow[b]{2}{*}{ Set } & \multirow[b]{2}{*}{ TS Orientation } & \multicolumn{2}{|c|}{ Zenith Angle, in DD MM SS } & \multirow[b]{2}{*}{ Measured slope distance, in $\mathrm{ft}$} \\
\hline & & & Measured & Reduced & \\
\hline 1 to 4 & \multirow[b]{2}{*}{1} & $\mathrm{~F} 1$ & $88^{\circ} 46^{\prime} 56^{\prime \prime}$ & $88^{\circ} 46^{\prime} 56^{\prime \prime}$ & 155.934 \\
\hline $\mathrm{HI}=4.850$ & & $\mathrm{~F} 2$ & $271^{\circ} 13^{\prime} 22^{\prime \prime}$ & $88^{\circ} 46^{\prime} 38^{\prime \prime}$ & 155.934 \\
\hline \multirow[t]{6}{*}{$\mathrm{SH}=5.040$} & \multirow[b]{2}{*}{2} & F1 & $88^{\circ} 46^{\prime} 56^{\prime \prime}$ & $88^{\circ} 46^{\prime} 56^{\prime \prime}$ & 155.931 \\
\hline & & F2 & $271^{\circ} 13^{\prime} 23^{\prime \prime}$ & $88^{\circ} 46^{\prime} 37^{\prime \prime}$ & 155.934 \\
\hline & \multirow{4}{*}{3} & F1 & $88^{\circ} 46^{\prime} 56^{\prime \prime}$ & $88^{\circ} 46^{\prime} 56^{\prime \prime}$ & 155.935 \\
\hline & & $\mathrm{F} 2$ & $271^{\circ} 13^{\prime} 23^{\prime \prime}$ & $88^{\circ} 46^{\prime} 37^{\prime \prime}$ & 155.934 \\
\hline & & & Average & $88^{\circ} 46^{\prime} 47^{\prime \prime}$ & 155.934 \\
\hline & & & Mark-to-mark VD & 3.131 & \\
\hline
\end{tabular}

5) Calculate the vertical difference $(V D)$ from the averaged zenith angle $(Z A)$ and slope distance $(S D)$ using this equation: $V D=S D \times(\cos Z A)$

6) Calculate the mark-to-mark VD using this equation: $V D$ (mark-to-mark) $=H I+V D-S H$

7) Sum the mark-to-mark $V D$ for the reciprocal observations to obtain the section misclosure

8) Determine if the magnitude of the misclosure is the result of a systematic error or measurement blunder. If the misclosure is from systematic errors, proceed to step 9; if a measurement blunder is detected, consider releveling the section.

9) Multiply the misclosure (determined in step 7) by -1 ; then divide that value in half to determine the adjustment value.

\begin{tabular}{|c|c|c|c|c|c|c|}
\hline \multirow{2}{*}{$\begin{array}{l}\text { Endpoints } \\
\text { of section }\end{array}$} & \multirow{2}{*}{$\begin{array}{l}\text { Length of } \\
\text { section }\end{array}$} & \multicolumn{2}{|c|}{ VD (mark-to-mark) } & \multirow{2}{*}{$\begin{array}{c}\text { Section Misclosure } \\
\text { (Foresight + Reciprocal) }\end{array}$} & \multirow{2}{*}{$\begin{array}{l}\text { Adjustment } \\
\text { value }\end{array}$} & \multirow{2}{*}{$\begin{array}{l}\text { Preliminary VD } \\
\text { (mark-to-mark) }\end{array}$} \\
\hline & & Foresight & Reciprocal & & & \\
\hline 1,2 & 127.42 & -0.145 & 0.144 & \begin{tabular}{|l|}
-0.001 \\
\end{tabular} & 0.0005 & -0.1445 \\
\hline 2,3 & 331.1 & 2.185 & -2.165 & 0.020 & -0.0100 & 2.1750 \\
\hline 3,4 & 140.2 & 1.105 & -1.108 & -0.003 & 0.0015 & 1.1065 \\
\hline 4,1 & 155.52 & -3.136 & 3.131 & -0.005 & 0.0026 & -3.1334 \\
\hline
\end{tabular}

Figure 26. Field measurements of a single-run loop traverse and the procedure to calculate section misclosure from reciprocal observations, determine preliminary mark-to-mark vertical differences, and determine preliminary elevations. 
10) Add the adjustment value to the mark-to-mark VD for the foresight measurement to determine the preliminary mark-to-mark VD. Round the preliminary mark-to-mark VD to $0.001 \mathrm{ft}$. For DRST, repeat steps 1 through 10 for the backward running. For SRLT and OT, proceed to step 11.

11) Add the preliminary mark-to-mark $V D$ to the elevation of the fiducial survey mark to determine the preliminary elevation (for SRLT) of the subsequent survey marker. The preliminary elevation should be reported to $0.001 \mathrm{ft}$.

12) Repeat this process for all the survey markers in the traverse.

\begin{tabular}{|c|c|c|c|c|}
\hline $\begin{array}{c}\text { Survey } \\
\text { mark } \\
\text { identifier }\end{array}$ & Point Description & Fiducial Elevation & $\begin{array}{c}\text { Preliminary VD } \\
\text { (mark-to-mark) }\end{array}$ & $\begin{array}{c}\text { Preliminary } \\
\text { Elevation }\end{array}$ \\
\hline 1 & Fiducial survey mark at origin point & 1971.43 & not applicable & not applicable \\
\hline 2 & Set survey mark (forward run) & not applicable & -0.145 & 1971.285 \\
\hline 3 & Set survey mark (forward run) & not applicable & 2.175 & 1973.460 \\
\hline 4 & Set survey mark (forward run) & not applicable & 1.107 & 1974.567 \\
\hline 1 & Closeout to origin & not applicable & -3.133 & 1971.434 \\
\hline
\end{tabular}

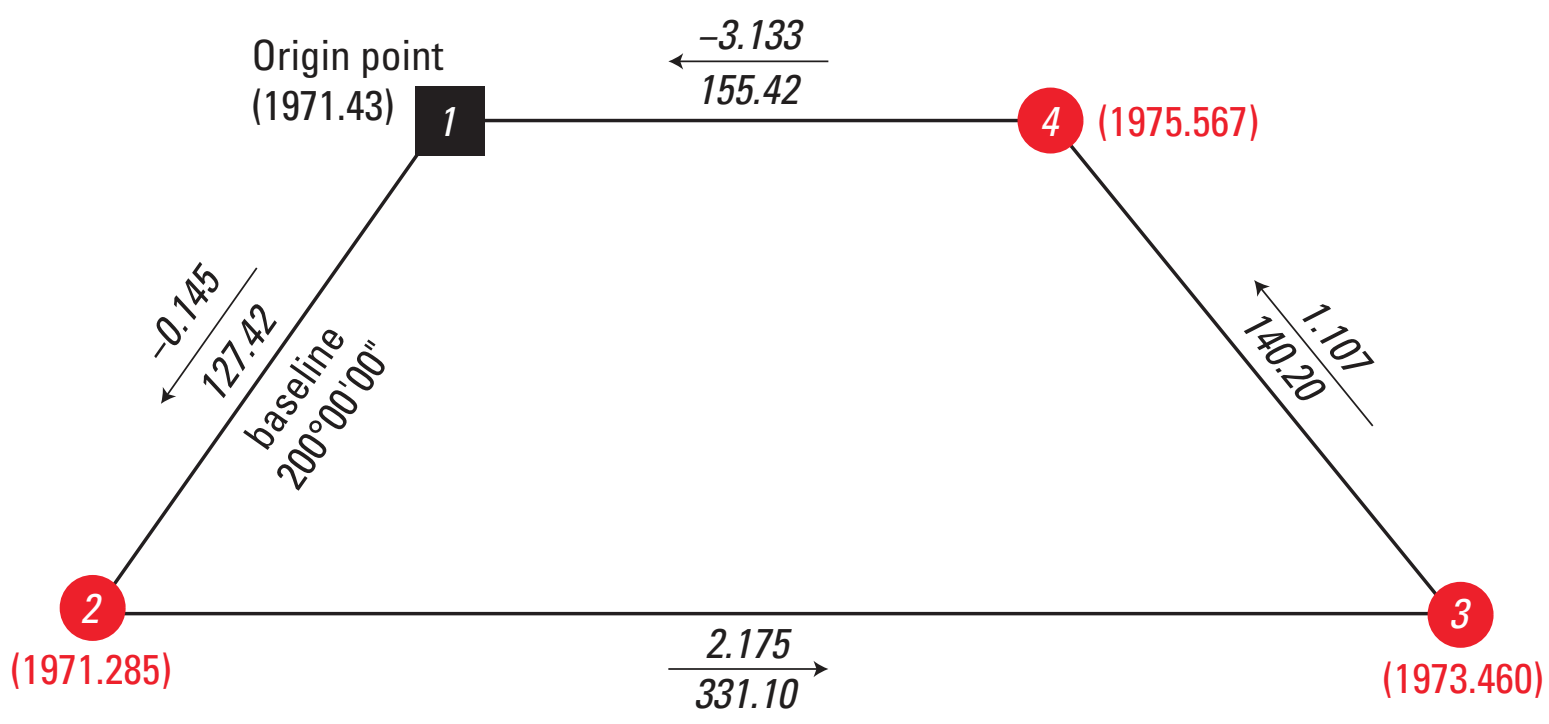

\footnotetext{
EXPLANATION

1 Fiducial survey marker-Inset number is the survey marker identifier.

(1971.43) Parenthetical indicates the elevation, in feet

104 Vertical control-Inset number is the survey marker identifier.

(19.661) Parenthetical upper number indicates preliminary elevation, in feet.

2.175
331.10 $\begin{aligned} & \text { Leveling leg (section)_Upper number indicates the preliminary } \\ & \text { mark-to-mark VD, in feet. Arrow indicates the direction of leveling. } \\ & \text { Lower number indicates horizontal distance between survey } \\ & \text { markers, in feet. }\end{aligned}$
}

Figure 26. Field measurements of a single-run loop traverse and the procedure to calculate section misclosure from reciprocal observations, determine preliminary mark-to-mark vertical differences, and determine preliminary elevations. 
10. Add the adjustment value to the (field measured) markto-mark VD for the foresight measurement to determine the preliminary mark-to-mark VD. The preliminary mark-to-mark VD should be rounded to $0.001 \mathrm{ft}$ using the rounding convention described by Hansen (1991). For DRST, repeat steps 1 through 10 for the backward running. For SRLT and OT, proceed to step 11.

11. Add the preliminary mark-to-mark VD for the foresight measurement to the elevation of the fiducial survey marker to determine the preliminary elevation (for SRLT) of the subsequent survey marker. The preliminary elevation should be reported to $0.001 \mathrm{ft}$.

12. Repeat this process for all the survey markers in the traverse.

The SRLT shown in figure 26 is an actual survey that was completed by the authors during a geospatial training activity in 2019. Instrument heights, zenith angles, and slope distances from multiple sets of F1 and F2 observations were tabulated from the exported job file to demonstrate section closure analysis (fig. 26). A 2" total station equipped with a $3 \mathrm{~mm}+2 \mathrm{ppm}$ precision EDMI was used for the traverse. Redundant instrument heights were collected at the backsight, station, and foresight for each setup. At least three sets of F1 and F2 measurements were collected at each station. In some cases, the difference between F1 and F2 zenith angles exceeded the 10" error threshold that was set in the data collector; the suspected source of this exceedance was a tilt compensator (described in the equipment section of this report) that was damaged during shipping. This systematic error was overcome by observing in $\mathrm{F} 1$ and $\mathrm{F} 2$ instrument orientation.

The 4-sided SRLT had a total length of $751.1 \mathrm{ft}$, and section misclosure from reciprocal observations ranged from $-0.0005 \mathrm{ft}$ (between survey markers 1 and 2) to $0.02 \mathrm{ft}$ (between survey markers 2 and 3; fig. 26). Preliminary elevations were calculated for each survey marker in the traverse; the loop-closure error of the SRLT is $-0.004 \mathrm{ft}$. This is a good example of how measurement blunders can go undetected if section closure is not scrutinized by the survey crew. If the analyst or crew chief was using loop-closure error as the primary indicator of survey quality without examining the individual closures of the component sections, the apparent blunder between survey markers 2 and 3 would go undetected. The error in that section $(0.02 \mathrm{ft})$ was offset by small misclosures $(-0.001,-0.003$, and -0.005$)$ along the leveling route and was virtually undetectable when the survey terminated at the origin. If this was an actual survey to determine the datum for a field site, the erroneous section may have been releveled depending on the accuracy requirements of the project; for training purposes, it was not resurveyed to demonstrate (1) the importance of recording section misclosure in the field and, (2) how measurement errors in a traverse sometimes offset and can go undetected without scrutiny by the field crew.
Section misclosure should be documented during the survey in a field book or standard field form (app. 1) to quality assure leveling measurements and to detect blunders. Field measurements should be adjusted during postprocessing by an analyst who has scrutinized the survey data. Onboard software programs are widely available for data-collection devices that can facilitate this process by adjusting field measurements for misclosure in real time without the need for rigorous hand calculation. The crew chief should be of aware of this capability and consult the user manual to ensure proper usage. Human oversight of automated routines is critical to quality assure data collection and processing.

\section{Distribution of Loop-Closure Error}

The mark-to-mark VD from the field measurements of a SRLT should be adjusted for section misclosure of reciprocal observations to obtain a preliminary mark-to-mark VD. The preliminary mark-to-mark VD is used to determine a preliminary elevation, which should be computed for each control point in a SRLT (fig. 26). The LCE of a SRLT is determined by subtracting the preliminary elevation of the origin point from the known (fiducial) elevation. Because systematic errors are assumed to accumulate with distance, LCE is proportionally distributed to the survey markers in the vertical-control network based on distance from the origin point. The LCE is calculated using the following equation:

$$
L C E=e B M_{o}-e B M_{p},
$$

where

$$
\begin{aligned}
L C E & \text { is the loop-closure error, } \\
e B M_{O} & \text { is the known elevation of the origin point } \\
& \text { survey marker, and } \\
e B M_{p} & \text { is the preliminary elevation of the origin point } \\
& \text { survey marker. }
\end{aligned}
$$

To demonstrate the LCE distribution procedure, assume a hypothetical SRLT consisting of five vertical-control points (including the fiducial origin point) has a total length of $2,110.5 \mathrm{ft}$ and a LCE of $-0.03 \mathrm{ft}$ after the preliminary elevations were determined from the preliminary mark-to-mark VDs. This error should be proportionally distributed based on the horizontal distance from the origin point survey marker to the subject survey marker using the following equation:

$$
e_{\text {final }} i=([d] \times[L C E \div D])+e_{p} i,
$$

where

$$
\begin{aligned}
& e_{\text {final }} \text { is the final elevation for a survey marker } i \text {, } \\
& i \quad \text { is an integer that represents the survey marker } \\
& \text { identifier, } \\
& d \quad \text { is the horizontal distance from the origin } \\
& \text { point of the survey to the indicated survey } \\
& \text { marker along the leveling circuit, } \\
& L C E \text { is the loop-closure error, }
\end{aligned}
$$




$$
\begin{gathered}
D \quad \text { is the total horizontal distance of the leveling } \\
\text { circuit, and } \\
e_{p} \quad \text { is the preliminary elevation for survey } \\
\text { marker } i \text {. }
\end{gathered}
$$

A multiplier is used to distribute error that is defined as the ratio of the $L C E$ to the total horizontal distance of the leveling circuit $(D)$. The ratio $(L C E \div D)$ is then multiplied by the horizontal distance $(d)$ from the origin point of the survey to the survey marker that is to be adjusted. This product is then added to the preliminary survey marker elevation $\left(e_{p} i\right)$ to determine the final elevation $\left(e_{\text {final }} i\right)$. LCE distribution is proportional to the distance from the origin point because measurement error accumulates in this way.

The horizontal distance from the origin point, measured along the loop, to survey markers $2,3,4$, and 5 is $411.1,866.7$, $1,254.5,1,654.9 \mathrm{ft}$, respectively (table 7). The distance from the fiducial origin point to the closeout point is equal to the sum of all the distances along the levels circuit $(2,110.5 \mathrm{ft})$, so the LCE $(-0.03 \mathrm{ft})$ will be applied to the measured elevation, as opposed to a fraction of the LCE for other survey markers. The distribution of the LCE for survey markers 2, 3, 4, and 5 is $-0.006,-0.012,-0.018$, and $-0.024 \mathrm{ft}$, respectively (table 7). The negative sign indicates the adjustment value is subtracted from the preliminary elevation to obtain the final elevation. The final elevation should be rounded to $0.001 \mathrm{ft}$ using the rounding convention described by Hansen (1991).

\section{Determining Section Misclosure from Double-Run Observations}

As previously described, the mark-to-mark VD from the field measurements should be adjusted for section misclosure of reciprocal observations to obtain a preliminary mark-tomark VD - this should be done for the forward running and backward running of a DRST (fig. 27). The preliminary markto-mark VDs from the forward running should be compared to the preliminary mark-to-mark VDs from the backward running to ensure they do not exceed the specifications for the desired standard (described in subsequent sections). In general, if the preliminary mark-to-mark VD from the forward running is within third-order specifications $( \pm 0.015 \mathrm{ft}$ for a

Table 7. Preliminary and final elevations of a single-run loop traverse.

[-, not applicable. $]$

\begin{tabular}{lrccc}
\hline $\begin{array}{c}\text { Survey } \\
\text { marker } \\
\text { identifier }\end{array}$ & $\begin{array}{c}\text { Distance } \\
\text { to survey } \\
\text { marker, } \\
\text { in feet }\end{array}$ & $\begin{array}{c}\text { Preliminary } \\
\text { elevation, } \\
\text { in feet }\end{array}$ & $\begin{array}{c}\text { Applied } \\
\text { adjustment, } \\
\text { in feet }\end{array}$ & $\begin{array}{c}\text { Final elevation, } \\
\text { in feet }\end{array}$ \\
\hline 1 (fiducial) & - & - & - & 345.16 \\
2 & 411.1 & 365.55 & -0.006 & 365.544 \\
3 & 866.7 & 415.12 & -0.012 & 415.108 \\
4 & $1,254.5$ & 399.87 & -0.018 & 399.852 \\
5 & $1,654.9$ & 371.71 & -0.024 & 371.686 \\
\hline
\end{tabular}

$500 \mathrm{ft}$ measurement distance) of the preliminary mark-to-mark VD from the backward running, the double-run leveling of the subject section is considered satisfactory. Preliminary markto-mark VDs are then adjusted to determine the final mark-tomark VDs, which is described in the subsequent section.

The method to determine the final mark-to-mark VDs is similar to the method to determine the preliminary mark-tomark VDs except the section misclosure is calculated from the double-run observations instead of the reciprocal observations (fig. 27). Sum the preliminary mark-to-mark VDs from the forward running and backward running of each section of the spur - the magnitude of the VDs should be nearly identical but have opposite signs. If the sum is within the desired specification (described in a subsequent section), multiply it by -1 , and then divide that value in half to determine the adjustment value. For each section, add the adjustment value to the preliminary mark-to-mark VD for the forward running to determine the final mark-to-mark VD for the forward running. Beginning at the origin point, add the final mark-to-mark VD to the elevation of the fiducial survey marker to determine the final elevation of the subsequent survey marker. Repeat this process for all the survey markers in the traverse for the forward running of the DRST.

Once the preliminary mark-to-mark VDs have been calculated from the reciprocal field observations, the spur-closure error (SCE) should be determined to evaluate the general precision of the leveling (fig. 27). Unlike LCE, SCE is not distributed proportionally to the control points as a function of distance from the origin point-in fact, once determined, it is not distributed at all. The redundant occupation of control points during the backward running of the leveling may offset random and systematic errors that accumulate with distance from the origin point for a DRST. Systematic errors can be minimized with impeccable field procedures, and random errors tend to cancel in nature. Proportioning closure error to the control points of a DRST may exacerbate random and systematic errors in the leveling that have already been minimized from double-run leveling observations (Daniel J. Martin, Northeast Regional Geodetic Advisor, NGS, written commun., 2020). SCE can be determined using the following equation:

$$
S C E=\sum V D_{\text {mark-to-mark }(\text { forward })}+\sum V D_{\text {mark-to-mark }(\text { backward })}
$$

where

$$
\begin{aligned}
& S C E \quad \text { is the spur-closure error, in feet; } \\
& V D_{\text {mark-to-mark (forward) } \quad \text { is preliminary mark-to-mark }} \\
& \text { vertical difference for the forward running } \\
& \text { of the section, in feet; and } \\
& \begin{array}{r}
V D_{\text {mark-to-mark (backward) is the preliminary mark-to-mark }} \\
\text { vertical difference for the backward } \\
\text { running of the section, in feet. }
\end{array}
\end{aligned}
$$

SCE is an important metric to help determine measurement precision of a DRST. The quality of a trigonometric-leveling survey and corresponding specifications for maximum allowable spur-closure error, which is proportional to square root of the one-way spur length, is detailed in the following section. 


\section{A. Forward running}

Origin point

(250.64)

\section{1}

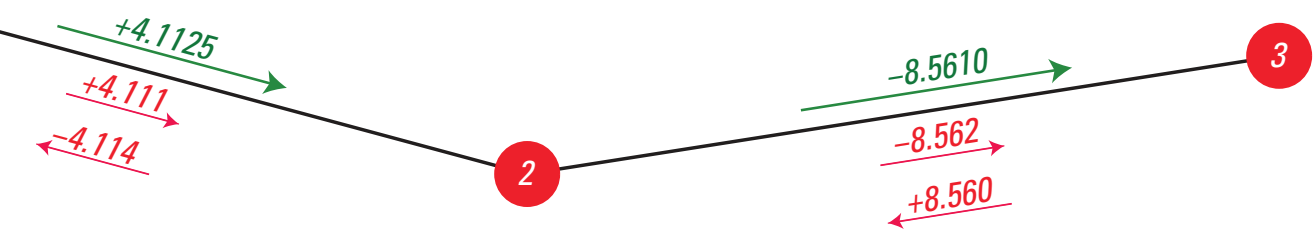

B. Backward running

Origin point

(250.64)

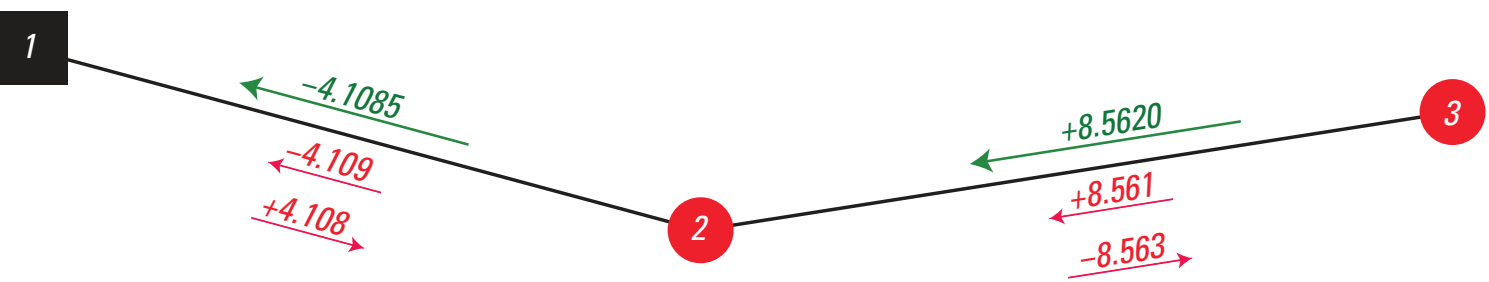

\section{Final elevations of control points}

Origin point

(250.64)

1
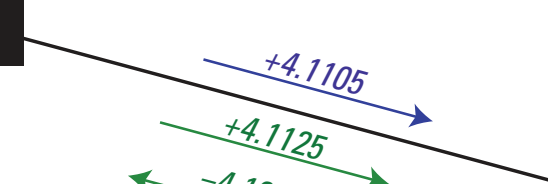

(254.751)

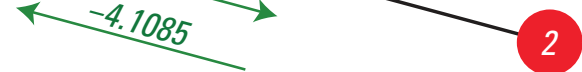

(246.189)

\section{EXPLANATION}

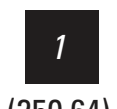

(250.64)

Fiducial survey marker-Inset number is the survey marker identifier. Parenthetical indicates the elevation, in feet

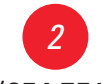

(254.751)

Vertical control-Inset number is the survey marker identifier. Parenthetical upper number indicates final elevation, in feet.
$+8.561$

$+8.56$

Reciprocal (field) measurement, in feet-Arrow indicates direction

Preliminary mark-to-mark VD, in feet-Arrow indicates direction

+8.561 Final mark-to-mark VD, in feet-Arrow indicates direction

$\overline{488.50}$ Leveling line (section)—Number indicates section length, in feet

Figure 27. Leveling process for a double-run spur traverse. $A$, reciprocal field measurements and preliminary mark-to-mark vertical difference (VD) for forward running of a double-run spur traverse (DRST); $B$, reciprocal field measurements and preliminary mark-to-mark VD for backward running of a DRST; $C$, final mark-to-mark VD and final elevations of control points; and $D$, method to compute spur-closure error. 


\section{Spur-closure error (SCE)}

Origin point

(250.64)

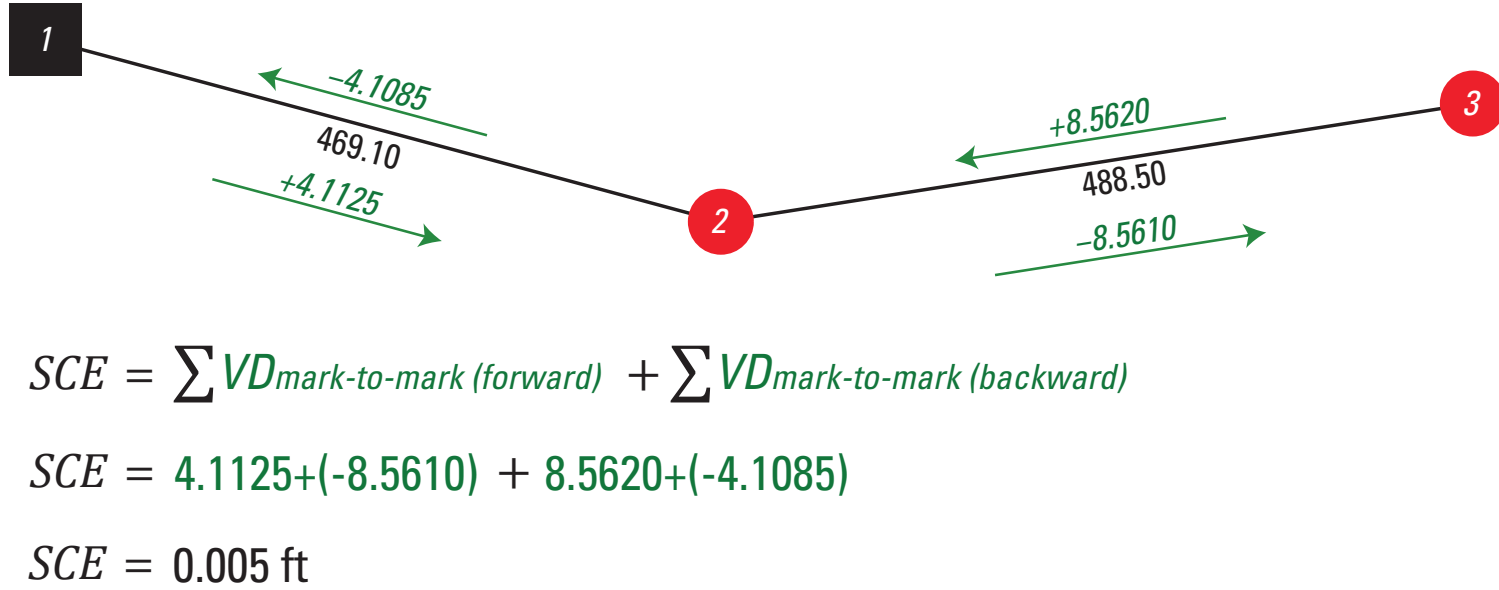

Figure 27. Leveling process for a double-run spur traverse. $A$, reciprocal field measurements and preliminary mark-to-mark vertical difference (VD) for forward running of a double-run spur traverse (DRST); $B$, reciprocal field measurements and preliminary mark-to-mark VD for backward running of a DRST; $C$, final mark-to-mark VD and final elevations of control points; and $D$, method to compute spur-closure error.

\section{Trigonometric-Leveling Quality}

A survey standard can be defined as the minimum accuracy needed to meet specific project objectives (Doyle and Kozlowski, 2013). For geodetic leveling, the NGS categorizes vertical-control points according to a classification scheme that is based on a standard error that is computed from a minimally constrained and correctly weighted least-squares adjustment (table 8; FGCC, 1984). The standard error, also called the maximum elevation difference accuracy, is the relative accuracy between directly connected vertical-control points, which is scaled by the square root of their horizontal separation traced along an existing leveling route (FGCC, 1984). When a vertical-control point is classified by the NGS, the orthometric elevation of that point is related by a specific accuracy to the other control points in the network. The NGS has developed specifications based on the accuracy standards for equipment, calibration schedules, and field procedures. For example, to achieve the $0.5-\mathrm{mm}$ accuracy standard for a 2-kilometer (km) leveling loop, the first-order (class I) specification for maximum allowable loop-closure error is $\pm 4.2 \mathrm{~mm}$ (table 8).

Table 8. Accuracy standards for vertical-control networks and corresponding example specifications. Modified from Federal Geographic Control Committee (1984) and Schomaker and Berry (1981).

[mm, millimeter; NGS, National Geodetic Survey; USGS, U.S. Geological Survey; ft, foot; $D$, one-way horizontal distances, in kilometers; $L$, one-way horizontal distance, in miles; - , NGS does not recognize classification]

\begin{tabular}{|c|c|c|c|c|c|c|}
\hline \multicolumn{2}{|c|}{ Classification } & \multirow[b]{2}{*}{$\begin{array}{c}\text { Standard } \\
\text { error, } \\
\text { in } \mathbf{~ m m}\end{array}$} & \multicolumn{2}{|c|}{ Example NGS specification } & \multicolumn{2}{|c|}{ Corresponding USGS specification } \\
\hline Order & Class & & $\begin{array}{l}\text { Maximum loop mis- } \\
\text { closure, in mm }\end{array}$ & $\begin{array}{l}\text { Maximum section } \\
\text { misclosure, } \\
\text { in } \mathrm{mm}\end{array}$ & $\begin{array}{c}\text { Maximum loop } \\
\text { misclosure, } \\
\text { in } \mathrm{ft}\end{array}$ & $\begin{array}{c}\text { Maximum section } \\
\text { misclosure, } \\
\text { in } \mathrm{ft}\end{array}$ \\
\hline \multirow[t]{2}{*}{ First order } & Class I & 0.5 & $3 \times \sqrt{D}$ & $4 \times \sqrt{D}$ & $0.010 \times \sqrt{ } L$ & $0.017 \times \sqrt{ } L$ \\
\hline & Class II & 0.7 & $4 \times \sqrt{ } D$ & $5 \times \sqrt{ } D$ & $0.017 \times \sqrt{ } L$ & $0.021 \times \sqrt{ } L$ \\
\hline \multirow[t]{2}{*}{ Second order } & Class I & 1.0 & $6 \times \sqrt{ } D$ & $6 \times \sqrt{ } D$ & $0.025 \times \sqrt{ } L$ & $0.025 \times \sqrt{ } L$ \\
\hline & Class II & 1.3 & $8 \times \sqrt{D}$ & $8 \times \sqrt{D}$ & $0.035 \times \sqrt{ } L$ & $0.035 \times \sqrt{ } L$ \\
\hline Third order & No classes & 2.0 & $12 \times \sqrt{ } D$ & $12 \times \sqrt{ } D$ & $0.050 \times \sqrt{ } L$ & $0.050 \times \sqrt{ } L$ \\
\hline Construction grade & No classes & - & - & - & $0.100 \times \sqrt{ } L$ & $0.100 \times \sqrt{ } L$ \\
\hline
\end{tabular}


The specifications that were developed by the NGS for geodetic leveling (Schomaker and Berry, 1981) have been adapted by the USGS for the purpose of developing standards for trigonometric leveling, which are identified as USGS Trigonometric Level I (TL I), USGS Trigonometric Level II (TL II), USGS Trigonometric Level III (TL III), and USGS Trigonometric Level IV (TL IV). These categories are necessary to communicate the quality of a survey to cooperating agencies, stakeholders, supervisors, reviewers, and other USGS surveyors to ensure the accuracy requirements of the project. A TL I survey is regarded as the highest quality survey that may be engaged by USGS staff; the specifications for field procedures become less rigorous with each subsequent category. In general, the TL I, TL II, and TL III categories have a combination of first-, second-, and third-order geodetic leveling specifications that have been modified and adapted for plane leveling. The TL III category also has specifications that are adapted from construction-grade standards (FGDC, 1998; USACE, 2007), which are not recognized by the NGS for geodetic leveling. A TL IV survey represents a leveling approach that does not generally meet criteria of a TL I, TL II, or TL III survey. The equipment specifications and calibration schedules listed in table 5 are identical for all categories; the previously described methods to establish an origin point, datum, and baseline for trigonometric-leveling surveys are also identical. The specifications of field procedures for each standard (category) are summarized in table 9 and are detailed in subsequent sections.

\section{Trigonometric Level I (TL I)}

The TL I survey is regarded as the highest quality survey that may be engaged by USGS staff. Closed traverse is required for this category to ensure surveys begin and terminate at the fiducial survey marker at the origin point. The specifications for this category are adapted from first- and second-order accuracy standards (tables 8 and 9; Schomaker and Berry, 1981; FGCC, 1984). The requirements are mostly identical for DRST and SRLT, except SRLT requires a minimum of two F1 and F2 measurement sets per station, and section misclosure from reciprocal observations must not exceed $0.005 \mathrm{ft}$. The specification for maximum section misclosure for reciprocal observations $(0.005 \mathrm{ft}$ for SRLT) is based on a first-order accuracy standard and may be difficult to attain at measurement distances approaching $500 \mathrm{ft}$ because of limitations from the hardware or the operator, or both. The simplest way to reduce misclosure of a section in the field (if blunders have been identified and eliminated) is to reduce the measurement distance between the station and the objective. The specifications are slightly more rigorous for SRLT as compared to DRST because DRST is generally regarded as a more meticulous approach because every control point in the network is occupied twice (once during the forward running and once during the backward running of the spur); maximum section misclosure from double-run observations must not exceed $0.01 \mathrm{ft}$. For both closed-traverse methods, the maximum difference between F1 and F2 zenith angles and slope distances cannot exceed $10^{\prime \prime}$ and $0.005 \mathrm{ft}$, respectively. Redundant instrument-height measurements ( $\mathrm{HI}$ and $\mathrm{SH}$ ) must also be within $0.005 \mathrm{ft}$. The specification for traverse closure error is identical for SCE and LCE and must not exceed $0.035 \mathrm{ft}$ multiplied by the square root of the length of the traverse (in miles). The surveyor should consider the quantity and distribution of the objectives when selecting a traverse approach (closed loop or spur). Effective planning is critical to ensure efficiency and the precision of the survey.

\section{Trigonometric Level II (TL II)}

The specifications for SRLT and DRST are identical for this category, so the surveyor should focus on the logistics of the traverse, the quantity of objectives, and how the objectives are distributed in space to select the most effective traverse approach. If the objective of a traverse is to perpetuate the datum to a single target some distance from the fiducial survey marker, a DRST could be a viable option because the most efficient route between two points is a line or a series of lines. A SRLT may be a more effective approach for multiple targets that are distributed in a nonlinear pattern that form the corners of a hypothetical polygon. The surveyor must also consider the quality of the survey; if the specifications are met, both of these surveys are considered TL II quality; however, the DRST is considered a slightly more precise approach because all the control points are occupied twice as opposed to a single occupation of control points (expect the origin point) for SRLT. However, if the time required to retrace the leveling lines back to the origin for the backward running of a DRST is much greater than the time it would take to establish a new path to the origin to close out the loop traverse, the potential incremental gain in measurement precision may be futile. Most of the specifications for the TL II category are adapted from the third-order accuracy standard (tables 8 and 9; Schomaker and Berry, 1981; FGCC, 1984). Redundant instrument-height measurements ( $\mathrm{HI}$ and $\mathrm{SH}$ ) must be within $0.005 \mathrm{ft}$, which is the only first-order specification for this category. The SCE and LCE for the DRST and SRLT, respectively, must not exceed $0.050 \mathrm{ft}$ multiplied by the square root of the length of the traverse (in miles).

\section{Trigonometric Level III (TL III)}

The TL III specifications for closed traverse are mostly adapted from the construction-grade standard (FGDC, 1998; USACE, 2007), which is similar to the closure error specification for rough terrain for differential levels at gaging stations (tables 8 and 9; Kenney, 2010). In addition to closed traverse, OT can also be used to establish vertical control and perpetuate the datum to field sites for the TL III standard. The specifications for OT are mostly adapted from the third-order accuracy standard for geodetic levels (tables 8 and 9; Schomaker and Berry, 1981; FGCC, 1984). The TL III 
Table 9. Trigonometric-leveling standards and corresponding specifications for field procedures.

[ft, foot; F1, total station is in direct instrument orientation; F2, total station is in reverse instrument orientation; +, excessive approach; HI, instrument height; $\mathrm{SH}$, height of sight; $S$, one-way spur length, in miles; NA, not applicable; $L$, length of loop, in kilometers; -, insufficient approach; $Z$, operating range of instrument]

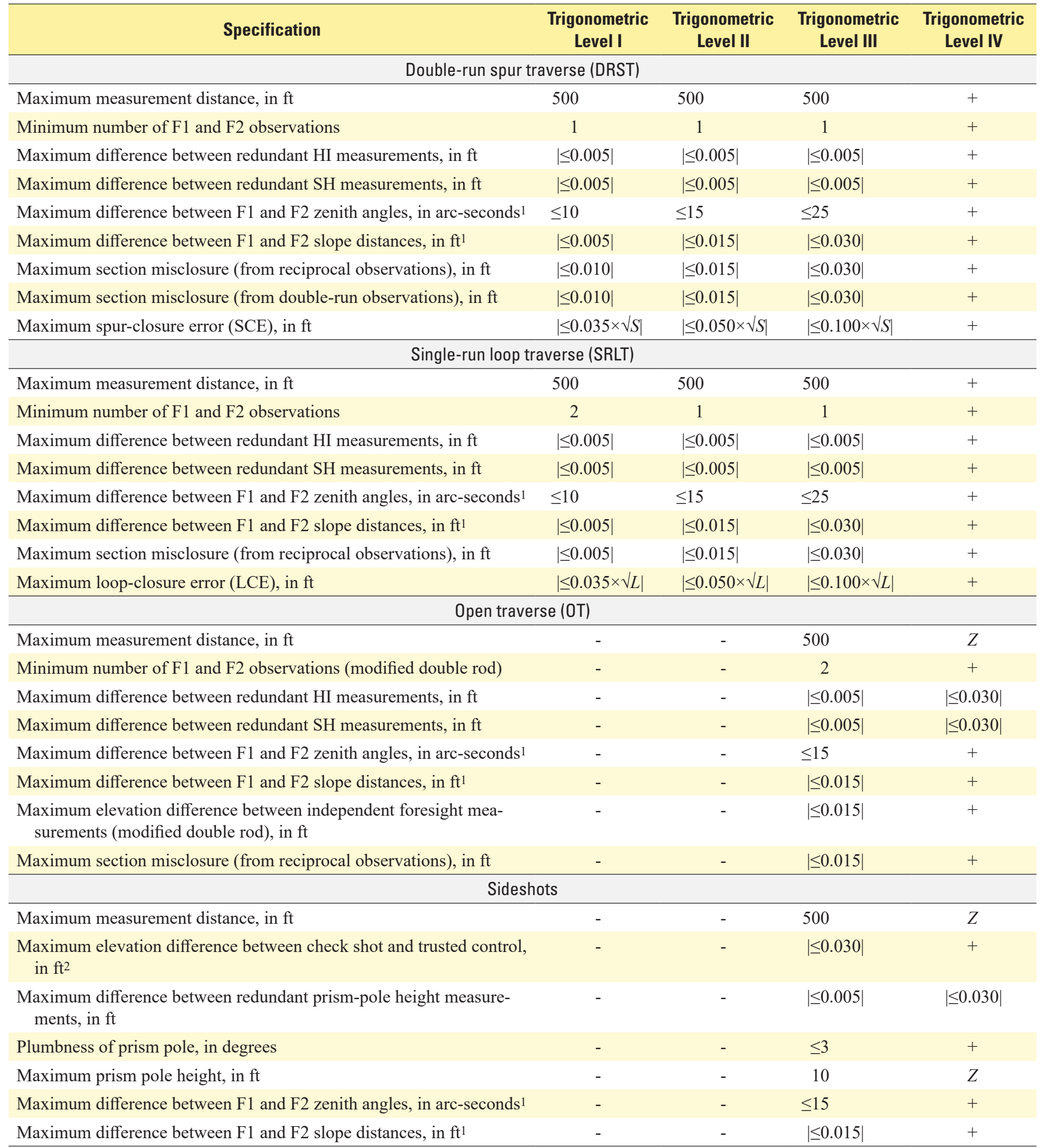

${ }_{1}^{1}$ Another set of F1 and F2 observations may be required if the specification is exceeded.

${ }^{2}$ Check shot measurement is observed in F1 only. 
specifications for OT are more rigorous than closed traverse because OT does not require a closeout to the origin point or redundant occupation of control points. Two sets of F1 and F2 measurements (modified double-rod method) are required for OT and must be within $0.015 \mathrm{ft}$. Sideshots may also be considered as TL III quality if they adhere to rigorous specifications listed in table 9; adapted third-order specifications are required for F1 and F2 observations, and F1 check shots to existing control must be within $0.03 \mathrm{ft}$ of the known value. The specifications for sideshots listed in table 9 are detailed in the subsequent "Sideshots" section of this manual. The TL III standard requires redundant instrument-height measurements (HI, SH, and prism pole [for sideshots]) that must be within $0.005 \mathrm{ft}$.

\section{Trigonometric Level IV (TL IV)}

Measurement distances are unrestricted for TL IV surveys; they are only limited by the maximum operating range of the equipment, which should be indicated on the specification sheet. The height of the prism pole used to obtain a sideshot is also unrestricted and is only limited by the range of the pole, which can be increased with additional extenders. The only restrictions for this category are the backsight check should be within $0.03 \mathrm{ft}$ (table 5), and redundant instrumentheight measurements for the $\mathrm{HI}, \mathrm{SH}$, and prism pole must also be within $0.03 \mathrm{ft}$ (table 9). Closed traverse is considered an excessive approach for a TL IV survey. Temporary survey markers, not permanent or semipermanent vertical control, should be set during a TL IV survey to ensure these marks will not be used in the future to perpetuate the datum.

The specifications listed in table 9 are estimates based on accuracy standards for vertical-control networks. An experienced analyst should use common sense and good judgment when evaluating and determining the quality of a trigonometric-leveling survey. For example, if every specification in a 6-section (leg) DRST is TL I quality except one exceedance that is TL II quality, the analyst may choose to rate the survey quality as TL I. Conversely, if the two-thirds of the specifications are TL II quality and the remaining third is TL 1 quality, the survey standard should be considered a TL II.

\section{Sideshots}

A sideshot is any radial measurement made with a total station that is not part of a traverse (fig. 28; USACE, 2007). Unlike traverse points (backsight, station, foresight), which are used to perpetuate the datum to field sites, sideshots are typically used for rapid, high-quantity data collection. Sideshots are also used to measure remote or inaccessible objective points that cannot be occupied with a total station. The measured elevation of a sideshot may have additional uncertainty because it is not part of the vertical-control network and is not subject to many of the rigorous quality-assurance procedures proposed in this manual. Sideshots are typically collected from a station in the control network, so the quality of the sideshot is considerably dependent on the quality of the traverse. Sideshots are usually measured with a prism pole, or a bipodmounted prism pole, which can also increase measurement uncertainty because the centering and level of the prism over the objective point is dependent on the skill and experience of the rodman and the adjustment of the surveying instrument(s). Prism poles are usually adjustable, so prism-pole heights can vary from about 5 to $10 \mathrm{ft}$ depending on the range of the pole. In some cases, prism-pole heights can be less than $0.5 \mathrm{ft}$ if the surveyor removes the prism from the prism pole to measure features such as high-water mark nails or to obtain topography measurements when the line of sight is obstructed. The variability of the prism-pole heights, combined with the increased potential for operator error, make sideshot measurements a relatively uncertain method to obtain quality elevations of objective points. Figure 28 indicates four sideshot surveys completed along the leveling route of an OT. The accuracy requirements of a project inform the appropriate quality assurance for a sideshot measurement.

Sideshot measurements are made by USGS surveyors for a variety of applications that include (but are not limited to) measuring high-water marks that correspond to peak-flood stage, which are used for flood and inundation modeling/mapping; completing stream-channel longitudinal-profile surveys, cross sections, and bridge and culvert geometry surveys that are used to determine discharge using indirect methods, for hydraulic modeling, and for geomorphology and sediment studies; measuring point and wellhead surveys used to reference water levels to a common datum and facilitate water table and potentiometric surface maps and electronic coverages; measuring bathymetry; inspecting cableway to determine sag and anchor stability; doing hydraulic control surveys; and surveying a glacier terminus. Because of the prevalent use of total stations in the USGS for purposes other than establishing vertical-control networks, and more specifically the need for three-dimensional (XYZ) data collection, quality-assurance recommendations for sideshot measurements are proposed in the subsequent section.

If a precise elevation is required for a sideshot, the survey crew chief should follow the specifications listed in table 9 for the TL III category. Unlike traverse surveys, F1 and F2 measurements for sideshots are observed to the foresight only (as opposed to backsight and foresight for traverse). The difference between F1 and F2 zenith angles and slope distances should not exceed $15^{\prime \prime}$ and $0.015 \mathrm{ft}$, respectively. Requirements for F1 and F2 horizontal angles is beyond the scope of this manual and should be determined by the survey crew chief. Before the survey, the height of the prism pole should be checked twice by the operator, and measurements should be within $0.015 \mathrm{ft}$; the circular bubble level should be also be properly adjusted (fig. 5). For the TL III category, the prism pole should not be extended beyond $10 \mathrm{ft}$ and should align with the direction of gravity within $3^{\circ}$. 


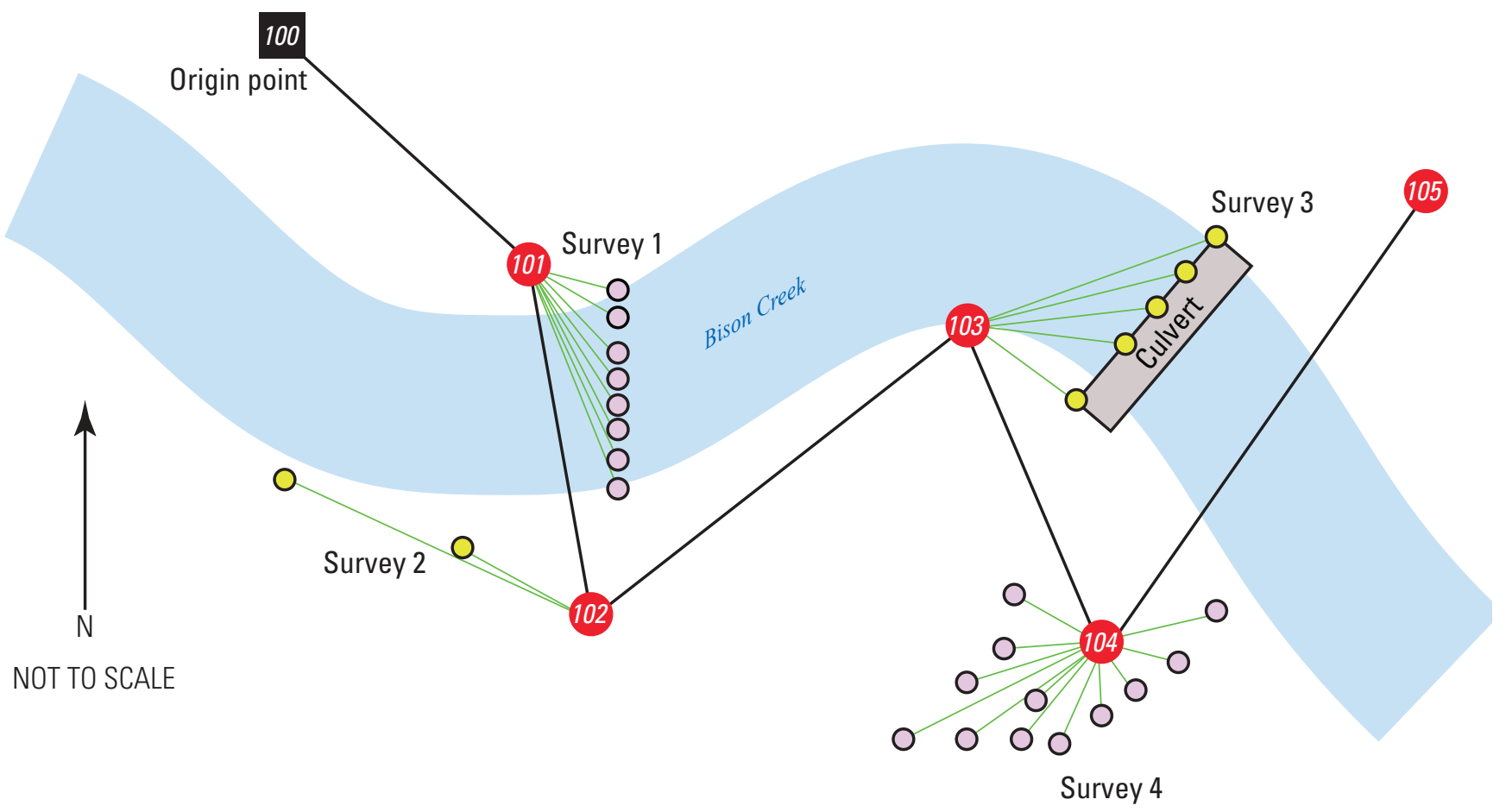

EXPLANATION

100 Fiducial survey marker-Inset number is the survey marker identifier.

102 Vertical control—Inset number is the survey marker identifier.

Sideshot-Direct and reverse instrument orientation (F1 and F2, respectively)

Sideshot-Direct instrument orientation (F1)

Line of sight from station to sideshot

Leveling leg (section)

Figure 28. Hypothetical open traverse and four sideshot surveys: survey 1, a cross section of Bison Creek with direct instrument orientation (F1) observations; survey 2, high-water mark measurements requiring F1 and reverse instrument orientation (F2) observations; survey 3, a culvert geometry survey, requiring F1 and F2 observations; and, survey 4, a topographic survey with F1 observations and check shots.

Similar to a backsight check, a "check shot" is used to verify the station setup, the total station is level and centered over the survey marker, and the instrument-height measurements are correct at the station and foresight. Check shots can be made quickly as part of the workflow during a sideshot survey without the need to configure the data-collection device for a formal backsight check; and can be collected at any control point in the traverse if the line of sight is unobstructed. Check shots are recommended when a large quantity of F1 sideshots are being collected from a single station. Check shots are observed in F1 only and should be made with the same instrumentation that is used to collect sideshot measurements; for example, if a prism pole is being used for the sideshot survey it should also be used to collect the check shot. The "stake-out" tool in the coordinate geometry program can facilitate the check-shot process, so the operator should consult the user manual regarding the workflow and functionality of this tool. The difference between the known and measured elevation for a check shot should not exceed $\pm 0.03 \mathrm{ft}$ for the
TL III category; for the TL IV category, check shots may be used to detect gross blunders such as an erroneous prismpole height. If sideshots are made from a station in a closed traverse, which require an elevation adjustment (described in the "Adjustments of Field Measurements" section), a vertical shift should be applied to the sideshots that is equivalent to the adjustment made at the station.

\section{Estimating Elevations of Remote Objective Points}

Some surveying projects may require elevations of remote objective points that cannot be accessed, or safely accessed, by the surveyor. There are a few methods the surveyor can use to estimate the elevation of an inaccessible feature or target; however, these types of measurements are considered extremely uncertain and should be used with caution. Many total stations have a "reflectorless" surveying mode, 
which is a misnomer because the signal that is transmitted from the instrument (usually a red or infrared laser) is reflected by an object (natural or manmade feature) and returned to the signal processing system of the total station (USACE, 2007). A more accurate identification of this survey method is "prismless" or "passive target" because a retroreflector (an active device used to precisely turn the signal back to its source with very little loss) is not needed to obtain a measurement. Prismless observations are used for estimating the position of features in remote locations that are not accessible or cannot be physically occupied by a survey crew or survey instrument; or in locations that pose a potential safety hazard for the survey crew. Prismless mode should be used with caution to estimate the location of an objective point because error can result if the instrument is not pointed correctly, or there are other reflectors near the objective point that may return an erroneous signal. It is also possible, if the instrument is out of adjustment, that the signal will not strike the object sighted with the crosshairs of the instrument. Common objects that can return a signal to the instrument include streets signs, utility poles, road striping, bridge abutments, building siding and roofs, and fences. Factors that affect measurement range and quality include the reflective properties of the target for the wavelength used by the instrument, target size and orientation (oblique versus orthogonal surfaces), and atmospheric and lightning conditions. Elevations that are obtained in prismless mode are considered uncertain and can be highly inaccurate, so the user is cautioned to use these data with care or not at all.

Some total stations do not have prismless functionality, so simple trigonometry can be used in conjunction with the total station and a graduated adjustable prism pole to estimate the heights (and elevations) of remote objects that have a surface that is orthogonal to the land surface. These features may include manmade structures such as buildings or dams or may include natural features such as trees, sheer rock faces, or bluffs. The critical part of this technique is to ensure the rodman is directly below the target, or as close to the plumb line as possible; and the total station, prism pole, and remote target are aligned horizontally to form three points of a triangle with its base at land surface (fig. 29). This method can also be used to measure objective features that are poor reflectors and cannot return a transmitted signal, or return an erroneous signal, to the EDMI signal processor.

The total station is set up some distance from the rodman in horizontal alignment with the prism pole and remote target. The total station is then leveled, and the zenith angle is set to $90^{\circ}$ and locked. After the $\mathrm{HI}$ is determined, the total station is pointed at the prism pole with the zenith angle still locked at $90^{\circ}$; the rodman adjusts the prism pole until the center (apex) of the prism is aligned with the crosshair of the telescope, which may require communication between the instrument operator and the rodman. Once the pole is properly adjusted, the rodman documents the pole height, and the instrument operator makes a horizontal distance measurement to the prism. Finally, the instrument operator points the telescope to the target above the rodman and records the zenith angle.
The height of the remote target, or VD between the horizontal plane of the prism and horizontal plane of the remote target, can be calculated using the following equation.

$$
V D_{r}=\tan \left(90^{\circ}-\mathrm{ZA}\right) \times H,
$$

where

$V D_{r} \quad$ is the height of the remote objective point above the plane of the surveying instruments,

$H \quad$ is the derived horizontal distance between total station and the prism, and

$Z A$ the measured zenith angle to the remote objective point.

The height of the remote target can be referenced to a vertical datum by establishing a survey marker of known elevation below the prism pole along the plumb line of the remote target. Add the known elevation of the survey marker to the prism-pole height and derived VD to obtain an estimate of the elevation of the remote target. Similar to prismless measurements, these are estimated observations, so the elevations obtained with this method should also be considered a rough estimate and used accordingly.

\section{Returning from the Field}

When a trigonometric-leveling campaign is complete, surveying instruments and accessories should be cleaned and stored according to manufacturer specifications and the procedures described in the "Trigonometric-Leveling Equipment" section of this manual. Coordinate and metadata files should be exported from the integrated microprocessor or data-collection device and archived in an electronic file that is replicated to a USGS server. Data files should be reviewed by an analyst to detect potential blunders and for quality assurance. If data files need to be adjusted or modified, a copy of the original file should be made to ensure the integrity of the original, authoritative dataset. Detailed electronic notes should be taken and stored in the modified file to document and justify changes and to identify the analyst. Hand-written field notes should be reviewed, scanned, and stored with the electronic files. Damaged and malfunctioning equipment should be documented in the field notes or metadata files to ensure appropriate actions are taken to repair faulty instrumentation in a timely manner. Defective equipment should be reported to other field crews to ensure the equipment is not used until it has been serviced and operating properly. A record should be maintained for each surveying instrument that identifies the equipment serial number and a record of service and calibration dates. 


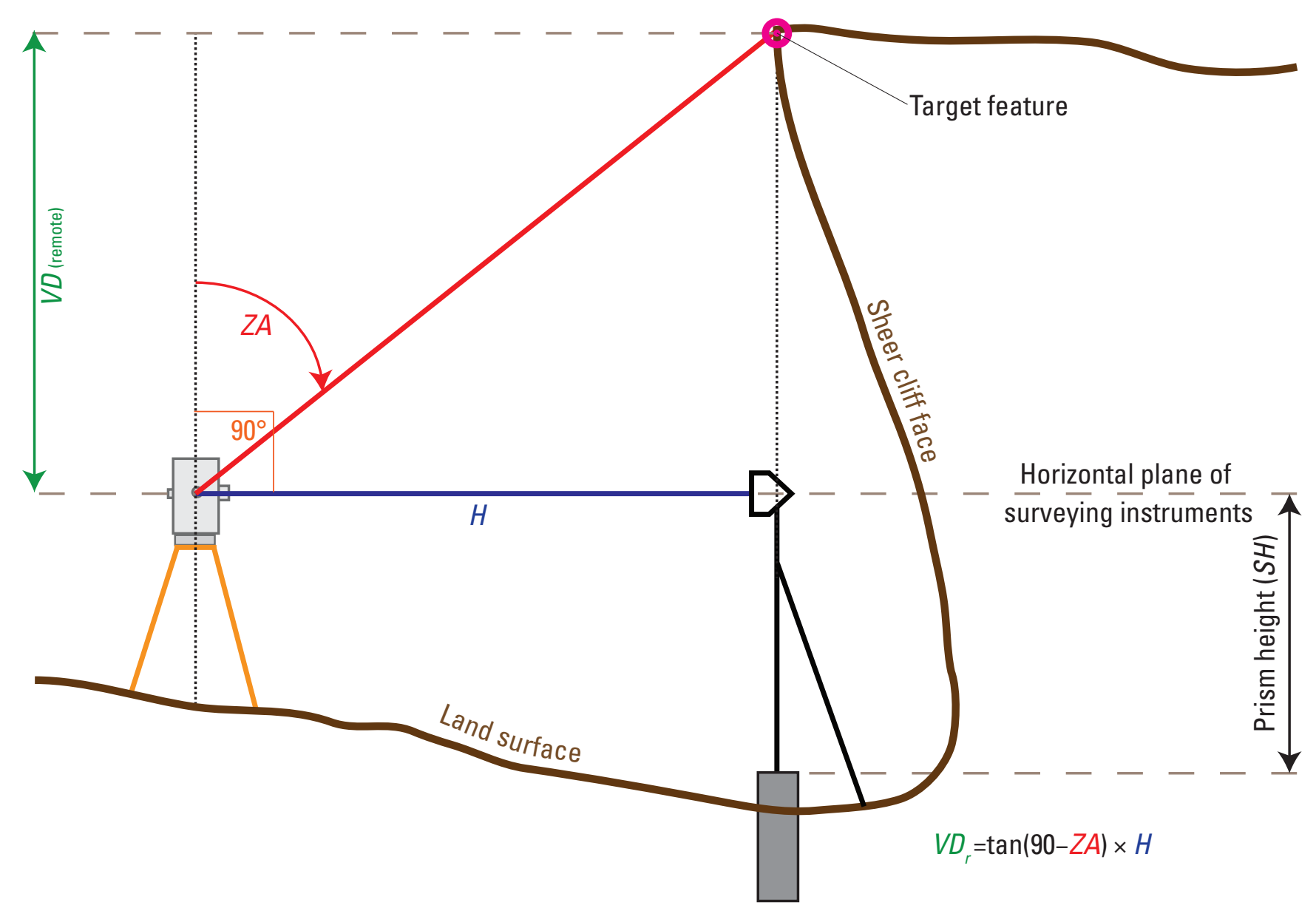

EXPLANATION
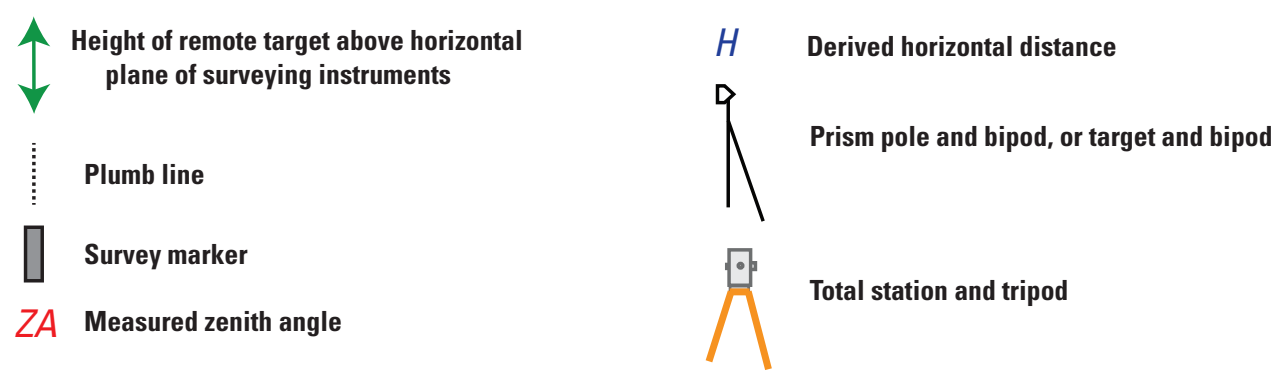

Figure 29. Estimating the elevation of remote objects using surveying instruments and simple trigonometry.

\section{Summary}

Total station instruments were developed in the 1980s and have become the workhorse in the land-surveying industry and a compelling alternative to conventional leveling for U.S. Geological Survey (USGS) surveyors for the purpose of establishing vertical-control networks and many other geospatial applications. The precision of a trigonometric-leveling survey essentially depends on four components (1) the skill and experience of the surveyor, (2) the environmental or site conditions, (3) the surveying method, and (4) the quality of the surveying instrumentation. Over the past 30 years, manufacturers have developed instrumentation capable of measuring horizontal and zenith angles at a precision of less than 1 arc-second, and distance measurements of 1 millimeter constant error and less than 2 parts per million scale error. A need exists to train USGS surveyors on the rigorous surveying protocol described in this manual because the precision of a land survey is no longer limited by the quality of the instrumentation, but by the experience and skill of the person operating the instrument. If USGS surveyors are properly trained and use quality instrumentation that is calibrated, 
well maintained and properly adjusted, first-, second-, and third-order accuracy standards can be routinely achieved to establish vertical-control networks and perpetuate a datum for project applications.

Having a basic understanding of land-surveying equipment operation, components, and capabilities is critical to collecting quality trigonometric-leveling data. Surveyors often need to make field adjustments or troubleshoot surveying equipment if malfunctioning equipment is detected or indicated by erroneous data. Surveying equipment must be tested before a trigonometric-leveling campaign to ensure quality observations and reduce measurement error related to the numerous sources of uncertainty that may be encountered when operating a total station and accessory equipment. These protocols include adjusting the leveling bubbles, adjusting optical and laser plummets, testing for axial defects, and testing the accuracy of the electronic distance measurement instrument on a calibrated baseline. Averaging zenith angles and slope distances observed in direct and reverse instrument orientation (F1 and F2), multiple sets of reciprocal observations, accurate meteorological observations to correct for the effects of atmospheric refraction, and electronic distance measurements that generally do not exceed 500 feet are common field procedures used to improve the quality of a trigonometric-leveling survey.

Closed traverse is considered the most effective way to perpetuate the datum because surveys are required to begin and terminate at the origin point of the survey, which provides a check on the accumulation of systematic errors in the traverse and will identify blunders that have gone previously undetected. Two types of closed traverse - double-run spur and single-run loop-are recommended in this manual because these approaches optimize measurement precision through rigorous quality assurance while providing the surveyor a choice to accommodate project logistics such as the quantity and spatial distribution of objectives. Adjustments to field measurements are made during postprocessing to refine the results of the survey before dissemination. The open traverse method was developed to accommodate control surveys for project sites where traversing back to the origin point may be impractical or potentially risk the safety of the survey crew. Regardless of traverse type, measurement precision can be optimized if the leap-frog surveying method described in this manual is executed by an experienced surveyor. Also identified as "forced centering," this technique may substantially increase work efficiency while reducing measurement uncertainty during long traverses that require many instrument setups and can be accomplished with any precisely adjusted traverse kit (total station, prisms, tribrach, and tripods). Classification schemes are necessary to communicate the uncertainty of a survey to cooperating agencies, stakeholders, supervisors, reviewers, and other USGS surveyors to ensure accuracy requirements of the project datum. These categories, or standards, are identified as USGS Trigonometric Levels I,
II, III, and IV. The requirements for each standard have been adapted for plane leveling from National Geodetic Survey specifications for geodetic leveling.

Total stations are currently being used in the USGS for purposes other than establishing vertical-control networks. USGS surveyors are utilizing total stations for a variety of geospatial applications that may require three-dimensional data collection of radial measurements made from a single station. These sideshots are not considered vertical control and therefore are not subject to procedures and best practices described in this manual for traverse surveys. For this reason, quality-assurance recommendations for sideshots such as observations made in F1 and F2 and check shots to existing vertical control should be considered. Both excessive and deficient amounts of quality assurance can impair a surveying project, delay project deadlines, and erode the confidence of cooperating agencies and stakeholders. Therefore, the survey crew should understand the scope of the survey and accuracy requirements of the project prior to a field campaign to ensure time and resources are being properly allocated, and an appropriate amount of quality assurance is implemented.

\section{References Cited}

Benson, M.A., and Dalrymple, T., 1967, General field and office procedures for indirect measurements: U.S. Geological Survey Techniques of Water-Resources Investigations, book 3, chap. Al, $30 \mathrm{p}$. [Also available at https://doi.org/10.3133/twri03A1.]

Berntsen, 2017, Surveying prisms: Bernsten web page, accessed March 16, 2017, at https://www.berntsen.com/ Surveying/Survey-Prisms?utm_source=bing\&utm medium $=$ cpc\&utm_campaign $=$ Surveying\&utm_term $=$ survey $\% 20$ prisms\&utm_content=Survey $\% 20$ Prisms.

Bjelotomić, O., Grgić, I., and Bašić, T., eds., 2011, Prefatory accuracy of leveling figure closure by combined measurements of trigonometric and geometric leveling: INGEO 2011, 5th International Conference on Engineering Surveying, Brijuni, Croatia, September 22-24, 2011, accessed April 21, 2017, at https://www.researchgate.net/ publication/272309099_Prefatory_accuracy_of_leveling_ figure_closure_by_combined_measurements_of_ trigonometric_and_geometric_leveling.

Brinker, R.C., and Minnick, R., 1995, The surveying handbook ( $2 \mathrm{~d}$ ed.): New York, N.Y., Chapman and Hall, 971 p. [Also available at https://doi.org/10.1007/978-1-46152067-2.].

Bullock, M.L., and Warren, R.E., 1976, Electronic total station speeds survey operations-Palo Alto, Calif., Hewlett-Packard Company: Hewlett-Packard Journal, v. 27, no. 8 , p. 2-12. 
California Department of Transportation, 2015, Survey equipment technical publication: California Department of Transportation, accessed March 16, 2017, at https://dot.ca.gov/des/techpubs/.

Carlson, 2017, Carlson Surveyor2-Technical specifications: Carlson web page, accessed July 14, 2017, at https://www.carlsonsw.com/products/hardware/surveyor2/.

Ceylan, A., Inal, C., and Sanlioglu, I., 2005, Modern height determination techniques and comparison of accuracies: Conference Paper TS33.5, Pharaohs to Geoinformatics FIG Working Week 2005 and GSDI-8, Cairo, Egypt, April 16-21, 2005.

Childers, V., and Youngman, M., 2016, GRAV-D airborne campaign - Overview, progress, future: National Oceanic and Atmospheric Administration, Alaska Surveying and Mapping Conference, Anchorage Alaska, February 17, 2016, presentation, 58 slides, accessed June 10, 2016, at https://www.ngs.noaa.gov/web/science_edu/presentations library/.

Chrzanowski, A., 1977, Design and error analysis of surveying projects: University of New Brunswick, lecture notes no. 47.

Chrzanowski, A., 1989, Implementation of trigonometric height traversing in geodetic levelling of high precision: University of New Brunswick, Geodesy and Geomatics Engineering, Technical Report 142, accessed March 3, 2017, at http://www2.unb.ca/gge/Pubs/ TechnicalReports.html.

Clayton, J.C., 1947, Surveying theory and practice. John Wiley \& Sons, Inc.

Cothrun, C., 1994, The technical side-Prism offset: Institute of Survey Technology of Ontario, The Ontario Land Surveyor, Summer 1994, accessed March 16, 2018, at https://www.krcmar.ca/resource-articles/1994_Summer_ Prism\%200ffsets_1.pdf.

Cothrun, C., 1995, The technical side of dual axis compensators: Institute of Survey Technology of Ontario, The Ontario Land Surveyor, Spring 1995, accessed July 11, 2017, at https://www.krcmar.ca/resource-articles/1995_Spring_ Dual\%20Axis\%20Compensators_1.pdf.

Doyle, D., and Kozlowski, J., 2013, FGDC accuracy standards: New Jersey Society of Professional Land Surveyors, Atlantic City, February 6, 2013, 44 slide presentation.

Dracup, J.F., Fronczek, C.J., and Tomlinson, R.W., 2019, Establishment of calibration base lines: National Oceanic and Atmospheric Administration, Technical Memorandum NOS NGS 8, $36 \mathrm{p}$.
Federal Geographic Data Committee [FGDC], 1998, Geospatial positioning accuracy standards part 4Standards for Architecture, Engineering, Construction $(\mathrm{A} / \mathrm{E} / \mathrm{C})$ and Facility Management: Washington, D.C., Federal Geographic Data Committee, FGDCSTD-007.4-2002, 19 p.

Federal Geodetic Control Committee [FGCC], 1984, Standards and specifications for geodetic control networks: Rockville, Md., National Oceanic and Atmospheric Administration, accessed March 9, 2010, at https://www.ngs.noaa.gov/FGCS/tech_pub/1984-stds-specsgeodetic-control-networks.pdf.

Fronczek, C.J., 1977, Use of calibration base lines (rev. 1980): National Oceanic and Atmospheric Administration, Technical Memorandum NOS NGS-10, 38 p., accessed June 25, 2020, at https://geodesy.noaa.gov/library/pdfs/ NOAA_TM_NOS_NGS_0010.pdf.

Hansen, W.R., ed., 1991, Suggestions to authors of the reports of the United States Geological Survey (7th ed.): Washington, D.C., U.S. Government Printing Office, 289 p.

Henning, W.E., 2010, User guidelines for single base real time GNSS positioning: National Geodetic Survey, 151 p.

Kavanagh, B.F., 2004, Surveying with construction applications: Upper Saddle River, N.J., Pearson Prentice Hall, 658 p.

Kenney, T.A., 2010, Levels at gaging stations: U.S. Geological Survey Techniques and Methods, book 3, chap. A19, 60 p. [Also available at https://doi.org/10.3133/tm3A19.]

Kozlowski, J., 1998, Application-Modern total stations are levels, too: Professional Surveyor Magazine, November 1998, accessed March 25, 2017, at https://archives.profsurv.com/magazine/article.aspx?i=359.

Leica Geosystems, 2008, Leica TPS400 series user manual (ver. 5.0): Heerbrugg, Switzerland, Leica Geosystems AG, 161 p., accessed May 1, 2017, at http://www.surveyequipment.com/ PDFs/TPS400User_en.pdf. [Also available at http://www.engineeringsurveyor.com/software/leica/tps 400 user_manual_en.pdf.]

Leica Geosystems, 2010, Surveying tribrachs-White paperCharacteristics and influences: Heerbrugg, Switzerland, Leica Geosystems AG, 9 p., accessed November 2, 2017, at https://leica-geosystems.com/products/total-stations/ accessories/tribrachs.

Leica Geosystems, 2013, Surveying made easy: Heerbrugg, Switzerland, Leica Geosystems AG, Technical Brochure, 35 p., accessed April 25, 2017, at https://w3.leicageosystems.com/downloads123/zz/general/general/ brochures/Surveying_en.pdf. 
Leica Geosystems, 2015, ATRplus-White paper: Heerbrugg, Switzerland, Leica Geosystems AG, 11 p., accessed April 2, 2019, at https://globalsurvey.co.nz/wp-content/uploads/ 2014/10/ATRplus_WP.pdf.

Leica Geosystems, 2017a, Robotic vs. manual total stations-A Q\&A with Golden Construction: Leica Geosystems AG, BIM Learning Center web page, accessed July 26, 2017, at https://bimlearningcenter.com/robotic-vs-manual-totalstations-a-qa-with-golden-construction/.

Leica Geosystems, 2017b, Tripods: Leica Geosystems AG web page, accessed July 27, 2017, at https://leicageosystems.com/products/total-stations/accessories/tripods.

Leica Geosystems, 2017c, Leica Viva TS11 datasheet: Leica Geosystems AG, 3 p., accessed November 8, 2017 , at https://leica-geosystems.com/products/total-stations/ manual-total-stations/leica-viva-ts11. [Also available at https://eica-geosystems.com/en-us/products/total-stations/ manual-total-stations/leica-viva-ts11-flexline-plusmanual-ts.]

Martin, D., 2015, Update on GRAV-D and progress toward a new vertical datum: National Oceanic and Atmospheric Administration, RISPLS/RIGIS NGS Forum, Kingston, R.I., July 21, 2015, presentation, 94 slides, accessed June 10, 2016, at https://www.ngs.noaa.gov/web/science edu/presentations_library/.

McMillen, 1993, Nodal-point adjusting retroreflector prism and method: U.S. Patent publication number 5231539, published July 27, 1993, accessed July 18, 2017, at http://patft.uspto.gov/netacgi/nph-Parser? Sect $1=$ PTO1 $\&$ Sect $2=$ HITOFF $\& d=$ PALL $\& p=1 \& u=$ $\% 2$ Fnetahtml $\% 2$ FPTO $\% 2$ Fsrchnum.htm \&r=1\&f=G\&l= 50\&s1=5231539.PN.\&OS=PN/5231539\&RS=PN/ $5231539 \backslash$.

Mish, T., 2008, Double-rodded level circuits explained: Professional Surveyor Magazine, accessed August 2020 at https://archives.profsurv.com/magazine/article.aspx? $\mathrm{i}=2118$.

National Oceanic and Atmospheric Administration [NOAA], 2007, The GRAV-D project-Gravity for the redefinition of the American Vertical Datum: National Oceanic and Atmospheric Administration, 38 p., accessed June 10, 2016, at https://www.ngs.noaa.gov/GRAV-D/pubs.shtml.

National Oceanic and Atmospheric Administration [NOAA], 2017a, What is geodetic leveling?: National Oceanic and Atmospheric Administration web page, accessed April 28, 2017, at https://www.ngs.noaa.gov/heightmod/Leveling/.

National Oceanic and Atmospheric Administration [NOAA], 2017b, EDMI Calibration Base Line program: National Oceanic and Atmospheric Administration web page, accessed September 15, 2017, at https://www.ngs.noaa.gov/ CBLINES/.
National Oceanic and Atmospheric Administration [NOAA], 2018, Finding survey marks and datasheets: National Oceanic and Atmospheric Administration web page, accessed August 11, 2018, at https://www.ngs.noaa.gov/ datasheets/.

Neilson, P.J., and Pickering, W.J., 1980, Electro-optical scanning: United States patent, publication number US4216495A, published August 5, 1980, accessed July 11, 2017, at http://patft.uspto.gov/netacgi/nph. [Also available at https://patents.google.com/patent/US4216495.]

Nindl, D., and Mao, J., 2010, Surveying reflectors-White paper-Characteristics and influences: Heerbrugg, Switzerland, Leica Geosystems AG, accessed July 25, 2017 , at http:/www.surveyequipment.com/PDFs/leica-whitepaper-surveying-prisms.pdf.

Noll, M.L., and Chu, A., 2017, Detecting temporal change in land-surface altitude using robotic land-surveying techniques and geographic information system applications at an earthen dam site in southern Westchester County, New York: U.S. Geological Survey Open-File Report 2017-1028, 16 p. [Also available at https://doi.org/10.3133/ ofr20171028.].

Ogundare, J.O., 2016, Precision surveying-The principles and geomatics practice. John Wiley \& Sons, Inc., 648 p. [Also available at https://doi.org/10.1002/9781119147770.].

Paiva, J.V.R., 2016, Important instrumentation tips for your team: NYSAPLS First Friday Webinar, July 8, 2016, accessed April 1, 2018, at http://c.ymcdn.com/ sites/www.nysapls.org/resource/resmgr/Webinars/ Handout_07-2016_Instrumentat.pdf. [Also available at https://cdn.ymaws.com/www.nysapls.org/resource/resmgr/ Webinars/Handout_07-2016_Instrumentat.pdf.]

Poole, J.S., and Bilen, L., 1976, Designing efficiency into a digital processor for an analytical instrument-Palo Alto, Calif., Hewlett-Packard Company: Hewlett-Packard Journal, v. 27, no. 8, p. 13-19.

Porter, S.J., 2001, Tribrach device: U.S. patent publication number USRE37318E1, accessed July 27, 2017, at https://patents.google.com/patent/USRE37318.

Rüeger, J.M., 1990, Basic working principles of electronic distance meters, in Electronic distance measurement: Springer, Berlin, Heidelberg, accessed July 17, 2017, at https://doi.org/10.1007/978-3-642-97196-9_4.

Rydlund, P.H., Jr., and Noll, M.L., 2017, Vertical datum conversion process for the inland and coastal gage network located in the New England, Mid-Atlantic, and South Atlantic-Gulf hydrologic regions: U.S. Geological Survey Techniques and Methods, book 11, chap. B8, 29 p., https://doi.org/10.3133/tm11B8. 
Rydlund, P.H., Jr., and Densmore, B.K., 2012, Methods of practice and guidelines for using survey-grade global navigation satellite systems (GNSS) to establish vertical datum in the United States Geological Survey: U.S. Geological Survey Techniques and Methods, book 11, chap D1, 102 p. with appendixes, accessed July 19, 2016, at https://pubs.usgs.gov/tm/11d1/.

Schomaker, M.C., and Berry, R.M., 1981, Geodetic leveling: Rockville, Md., National Oceanic and Atmospheric Administration, National Ocean Survey, NOAA Manual NOS NGS 3, 162 p., accessed May 2020 at https://www.ngs.noaa.gov/PUBS_LIB/GeodeticLeveling_ Manual_NOS_NGS_3.pdf.

Smith, C.L., 2010, Bench mark reset procedures - Guidelines to preserve elevation data for a soon-to-be disturbed or soon-to-be destroyed bench mark: Silver Spring, Md., National Oceanic and Atmospheric Administration National Geodetic Survey, 24 p.

Smith, D.A., and Roman, D.R., 2010, How NOAA's GRAV-D project impacts and contributed to NOAA science: National Oceanic and Atmospheric Administration, 4 p., accessed June 10, 2016, at https://www.ngs.noaa.gov/GRAV-D/ pubs.shtml.
Topcon, 2018, Topcon reflectorless total station GPT-3500LN: Topcon web page, accessed March 14, 2018, at https://www.topcon.co.jp/en/positioning/products/product/ ts00/GPT-3500LN E.html.

Trimble, Inc., 2017, Trimble S-6 total station: Trimble, Inc., web page, accessed July 10, 2017, at http://www.trimble.com/Survey/trimbles6.aspx/.

U.S. Army Corps of Engineers [USACE], 2007, Engineering and design, Control and topographic surveying: U.S. Army Corps of Engineers Engineer Manual 1110-1-1005, 498 p.

U.S. Department of the Army, 1980, Operator's, organizational, direct support, and general support maintenance manual, theodolite surveying: U.S. Department of the Army TM 5-6675-312-14.

U.S. Department of the Army, 2001, Topographic surveying: U.S. Department of the Army Field Manual 3-34.331.

Vaniceck, P., and Krakiwsky, E.K., 1986, Geodesy-The concepts: Amsterdam, North Holland Publishing Co.

Whalem, C.T., 1985, Trigonometric motorized leveling at the National Geodetic Survey, in Proceedings of the Third International Symposium on the North American Vertical Datum, Rockville, Md., April 21-26, 1985: Rockville, Md., National Oceanic and Atmospheric Administration, p. $65-80$. 


\section{Appendix 1 Standard Field Form for Running Trigonometric Levels}

\section{U.S. Geological Survey \\ Trigonometric Leveling \\ Traverse Form}

Sheet___ of

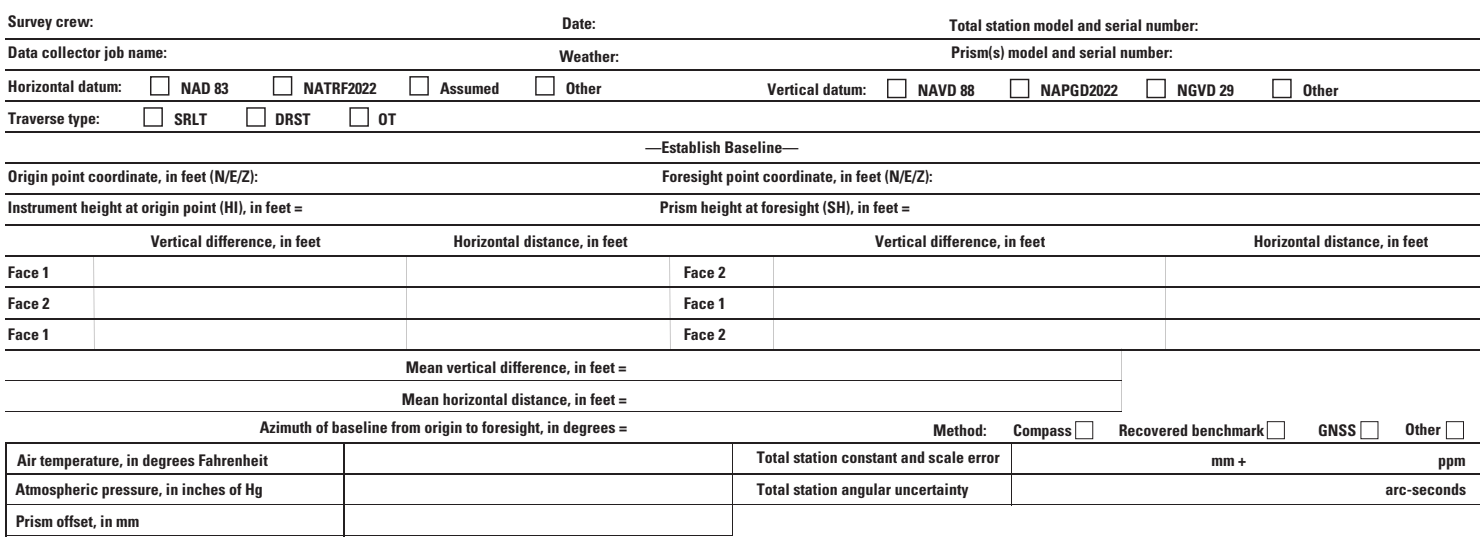

Helpful conversions: $I m=3.28084$ ff; $1 \mathrm{~mm}=0.00328084 f$ f: $I^{\circ} \mathrm{C}=1.8^{\circ} \mathrm{F} ; \mathrm{lmm}=0.03937008$ inches

Setup \# 1

\begin{tabular}{|l|l|l|l|l|}
\hline Backsight point number & BS prism height (SH), in feet $=$ & & \\
\hline Station point number & Instrument height (HI), in feet $=$ & & \\
\hline Foresight point number & FS prism height (SH), in feet $=$ & & \\
\hline
\end{tabular}

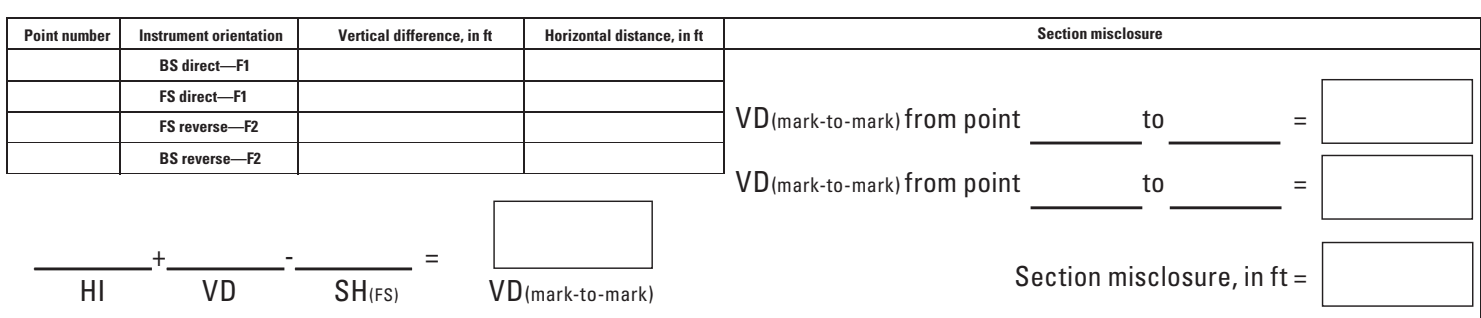

Setup \#- Description
\begin{tabular}{|l|r|r|l|l|}
\hline Backsight point number & BS prism height (SH), in feet $=$ & & \\
\hline Station point number & Instrument height $(\mathrm{HII})$, in feet $=$ & & \\
\hline Foresight point number & FS prism height (SH), in feet $=$ & & \\
\hline
\end{tabular}

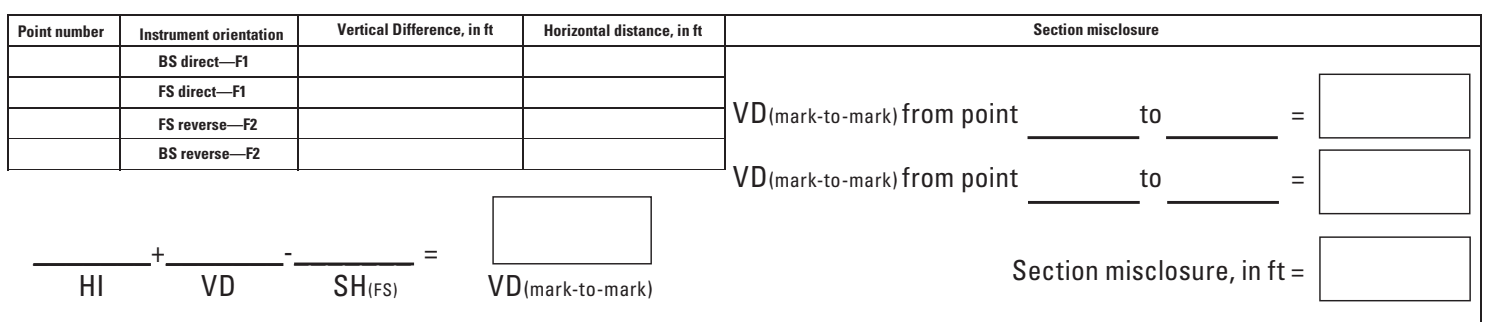

Setup \# _

Backsight point number

Station point number

Foresight point number

Description

\begin{tabular}{|c|c|c|c|c|c|c|}
\hline \multirow{2}{*}{ Point number } & \multirow{2}{*}{$\begin{array}{c}\text { Instrument orientation } \\
\text { BS direct-F1 }\end{array}$} & \multirow[t]{2}{*}{ Vertical Difference, in ft } & \multirow[t]{2}{*}{ Horizontal distance, in ft } & \multicolumn{3}{|c|}{ Section misclosure } \\
\hline & & & & \multirow{3}{*}{$\begin{array}{l}\text { VD (mark-to-mark) from point } \\
\text { VD (mark-to-mark) from point }\end{array}$} & \multirow{3}{*}{ to } & \multirow{3}{*}{$=$} \\
\hline & FS direct-F1 & & & & & \\
\hline & FS reverse $-\mathrm{F} 2$ & & & & & \\
\hline
\end{tabular}

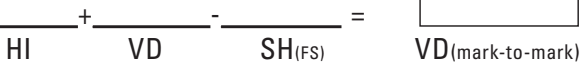

Section misclosure, in $\mathrm{ft}=$

Standard field form for running trigonometric levels. 


\section{Glossary}

blunder A gross error that may cause a measurement uncertainty specification to be exceeded. As opposed to systematic or random errors, blunders are less discrete and are commonly the result of a mistake by the operator during the data collection workflow.

compass-rule adjustment Distributes the linear misclosure of a traverse proportionally throughout each leg of a traverse. Also identified as the "Bowditch-rule adjustment."

corner-cube prism A solid piece of cylindrical optical glass that reflects the electromagnetic signal that is transmitted by the electronic distance measurement instrument.

critical angle The greatest angle at which a ray of light, travelling in one transparent medium, can strike the boundary between that medium and a second of lower refractive index without being totally reflected within the first medium.

face 1 (F1) Total station is in direct instrument orientation.

face 2 (F2) Total station is in reverse instrument orientation.

first-order (vertical) accuracy Is an assurance that the orthometric elevation of a control point bears a relation of specific accuracy to all the other control points in the network. For the first-order elevation accuracy standard, the maximum elevation difference accuracy is 0.7 millimeter between a pair of control points (pertains to all pairs in the network) that is scaled by the square root of their horizontal separation traced along existing level routes. The maximum elevation difference accuracy is computed from a minimally constrained and correctly weighted least squares adjustment. All subsequent first-order specifications are based off this standard.

second-order (vertical) accuracy Is an assurance that the orthometric elevation of a control point bears a relation of specific accuracy to all the other control points in the network. For the second-order elevation accuracy standard, the maximum elevation difference accuracy is 1.3 millimeters between a pair of control points (pertains to all pairs in the network) that is scaled by the square root of their horizontal separation traced along existing level routes. The maximum elevation difference accuracy is computed from a minimally constrained and correctly weighted least squares adjustment. All subsequent second-order specifications are based off this standard.

third-order (vertical) accuracy Is an assurance that the orthometric elevation of a control point bears a relation of specific accuracy to all the other control points in the network. For the third-order elevation accuracy standard, the maximum elevation difference accuracy is 2.0 millimeters between a pair of control points (pertains to all pairs in the network) that is scaled by the square root of their horizontal separation traced along existing level routes. The maximum elevation difference accuracy is computed from a minimally constrained and correctly weighted least squares adjustment. All subsequent third-order specifications are based off this standard.

fiducial benchmark A trusted physical marker of known elevation that references a vertical datum; typically used as the point of origin and closeout point for traverse surveys.

inverse tool A coordinate geometry function used to compute the bearing and distance (horizontal and vertical) between two points.

least-squares adjustment An adjustment in which the sum of the squares of the differences between given and changed positions are minimized. An adjustment that will tie all points together into a "best fit" network with the least amount of positional change.

modulation frequency Is the encoding of information in a carrier wave by varying the instantaneous frequency of the wave.

open traverse A trigonometric-leveling survey used to establish vertical control that originates at a point of known location and terminates at a point of unknown location.

refractive index The ratio of the velocity of light in a vacuum to its velocity in a specified medium. 
resection A method for determining an unknown position measuring angles with respect to known positions.

retroreflector See corner-cube prism.

rotation A type of mathematical transformation that involves the movement of a group of points around a fixed point.

setup General term used to describe the location and associated point number of the backsight, station, and foresight during a traverse survey.

station The ground point or survey marker that is being occupied by the total station.

station setup Used to describe the menu in the data-collection device where setup information is entered which includes the point numbers and measured heights for the backsight and station.

transformation A transformation is the change in shape or size of a figure or group of points.

translation A type of mathematical transformation that involves shifting a group of points from one place to another without rotating, reflecting, or changing size or scale.

traverse point The backsight, station, or foresight (or all) in a traverse survey.

vertical control $A$ trusted physical marker of known elevation that references a vertical datum and typically established during traverse surveys. 

For more information about this report, contact: Director, New York Water Science Center

U.S. Geological Survey

425 Jordan Road

Troy, NY 12180-8349

dc_ny@usgs.gov

518-285-5602

or visit our website at https://www.usgs.gov/centers/ny-water Publishing support provided by the

Pembroke and Rolla Publishing Service Centers 
